\title{
The effect of financial retirement incentives originating from the social security system on the retirement behavior of older Belgian workers
}

Citation for published version (APA):

Fraikin, A-L. (2021). The effect of financial retirement incentives originating from the social security system on the retirement behavior of older Belgian workers. [Doctoral Thesis, Maastricht University].

ProefschriftMaken. https://doi.org/10.26481/dis.20210902af

Document status and date:

Published: 01/01/2021

DOI:

10.26481/dis.20210902af

Document Version:

Publisher's PDF, also known as Version of record

Please check the document version of this publication:

- A submitted manuscript is the version of the article upon submission and before peer-review. There can be important differences between the submitted version and the official published version of record.

People interested in the research are advised to contact the author for the final version of the publication, or visit the DOI to the publisher's website.

- The final author version and the galley proof are versions of the publication after peer review.

- The final published version features the final layout of the paper including the volume, issue and page numbers.

Link to publication

\footnotetext{
General rights rights.

- You may freely distribute the URL identifying the publication in the public portal. please follow below link for the End User Agreement:

www.umlib.nl/taverne-license

Take down policy

If you believe that this document breaches copyright please contact us at:

repository@maastrichtuniversity.nl

providing details and we will investigate your claim.
}

Copyright and moral rights for the publications made accessible in the public portal are retained by the authors and/or other copyright owners and it is a condition of accessing publications that users recognise and abide by the legal requirements associated with these

- Users may download and print one copy of any publication from the public portal for the purpose of private study or research.

- You may not further distribute the material or use it for any profit-making activity or commercial gain

If the publication is distributed under the terms of Article $25 \mathrm{fa}$ of the Dutch Copyright Act, indicated by the "Taverne" license above, 


\section{THE EFFECT OF FINANCIAL RETIREMENT INCENTIVES ORIGINATING FROM THE SOCIAL SECURITY SYSTEM ON THE RETIREMENT BEHAVIOR OF OLDER BELGIAN WORKERS}

Anne-Lore Fraikin 


\section{THE EFFECT OF FINANCIAL RETIREMENT INCENTIVES ORIGINATING FROM THE SOCIAL SECURITY SYSTEM ON THE RETIREMENT BEHAVIOR OF OLDER BELGIAN WORKERS}

\section{DISSERTATION}

to obtain the degree of Doctor at Maastricht and Liege University, on the authority of the Rector Magnificus Prof. Dr. Rianne M. Letschert in accordance with the decision of the Board of Deans to be defended in public on Thursday, 2 September 2021 at 16:00 hours.

By

ANNE-LORE FRAIKIN 


\section{Supervisors:}

Prof. Dr. Alain Jousten, Liege University

Prof. Dr. Alessio Brown, Maastricht University

\section{Assessment Committee:}

Prof. Dr. Klaus Zimmerman (Chair), Maastricht University

Prof. Dr. Antoine Bozio, EHESS and Paris School of Economics

Prof. Dr. Corrado Giulietti, University of Southampton

Prof. Dr. Mathieu Lefebvre, Liege University

Dr. Zina Nimeh, Maastricht University

Prof. Dr. Sergio Perelman, Liege University 
Cover Design: ProefschriftMaken

Publisher: ProefschriftMaken II www.proefschriftmaken.nl

ISBN: 978-94-6423-398-8

Copyright @ Anne-Lore Fraikin, 2021.

All rights reserved. No part of this publication may be reproduced, stored in a retrieval system, or transmitted in any form, or by any means, electronic, mechanical, photocopying, recording or otherwise, without the prior permission in writing, from the author. 


\section{ACKNOWLEDGEMENTS}

First and foremost, I want to express my deepest gratitude to my promoter Alain Jousten for his guidance and invaluable support throughout my Ph.D. His brilliant and sharp mind, his exceptional analytical skills and his attention for detail, have truly been an inspiration. I have looked up to these qualities for the past 5 years. I was very fortunate to have such an eminent promoter, thanks to whom I have become an accomplished researcher in economics. I am very grateful for your patience and for your many comments and advice on my work, as well as for the friendly conversations we have had over the years.

I also want to give my thanks to my supervisor, Alessio Brown. I am most grateful for his many positive comments and feedback over the years, which have helped me to keep my head straight and to complete my manuscript. His insightful comments have assisted me in challenging my work and research questions to keep them coherent. Thank you for your ceaseless encouragements, which have given me the reassurance and the confidence I needed to bring my thesis to a conclusion.

My deep appreciation goes to the other members of my assessment committee. Thank you for accepting to evaluate my work and for participating in my thesis defense. I would like to give special thanks to Sergio Perelman, for his many sagacious comments and feedback, which have helped me tremendously to improve the quality of my work. I am also most grateful for the support and comments I received from Mathieu Lefèbvre during the NBER International Social Security project. Thank you to Zina Nimeh for introducing me to social protection and for being such an amazing counselor over the years. I also would like to thank Daniel Janssens, for his assistance in reviewing my work over the years. Finally, thank you to my colleagues from Liege University and to the members of 
the 2016 UNU-MERIT PhD Cohort for the feedback and many lively events. I must give special thanks to Anny Yu, who has been an invaluable support over the years and keeps being one of my most trusted friends.

Lastly, my parents deserve endless gratitude for encouraging me and for giving me the opportunity to follow my dreams. Without you, I would not be where I am today. I am forever indebted to my father, Luc, who has been my strongest supporter over the years and has not once stopped believing in me. I will always be grateful for the unconditional love and affection from my mother, Véronique. I must also give thanks to my grandparents, Mariette, François and Helda, who have shaped my personality and helped me become who I am today. Thank you for the encouragements expressed by my brother and sister, Rémy and Élodie, and by my friends. Last but not least, I thank my husband, Benjamin, and my wonderful daughter, Apolline, for helping me to keep my feet firmly on the ground and for surrounding my with undying love. 


\section{TABLE OF CONTENT}

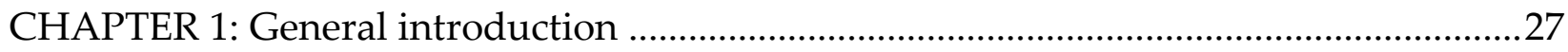

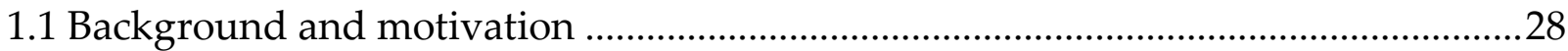

1.2 The retirement behavior and the social security system ………..................................31

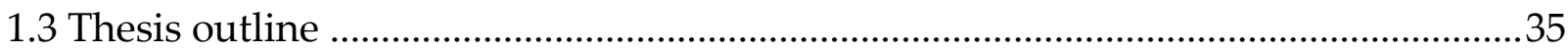

CHAPTER TWO: Historical evolution of the Belgian social security landscape for older

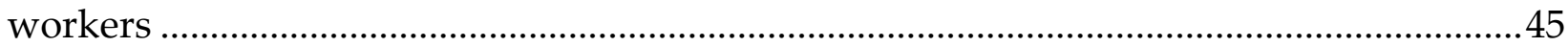

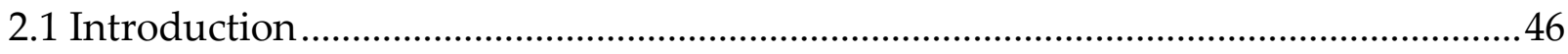

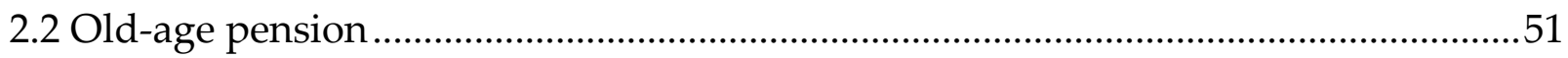

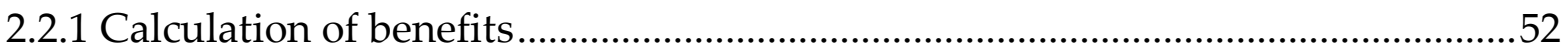

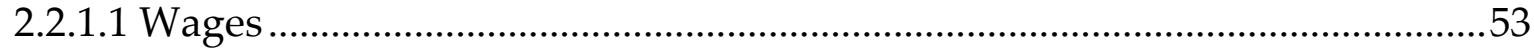

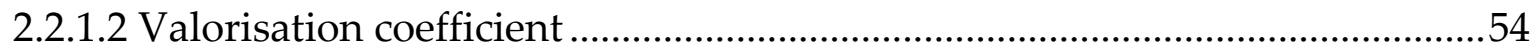

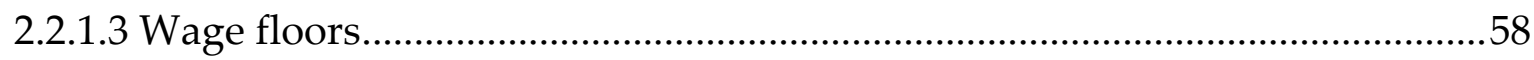

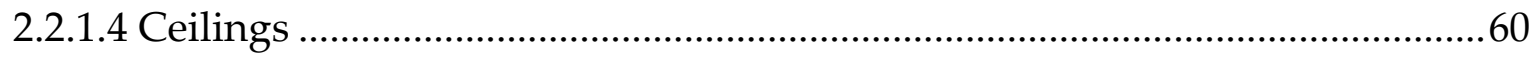

2.2.1.5 Replacement rate ..........................................................................................62

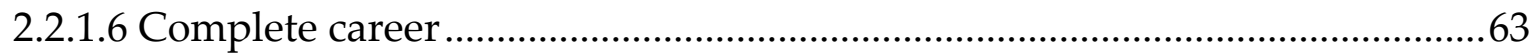

2.2.2 Statutory and early eligibility ages........................................................................64

2.2.3 Adjustments of pension amounts ..........................................................................67

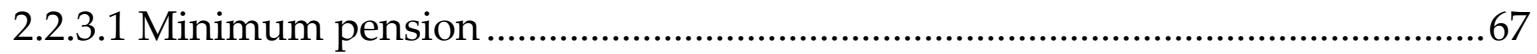

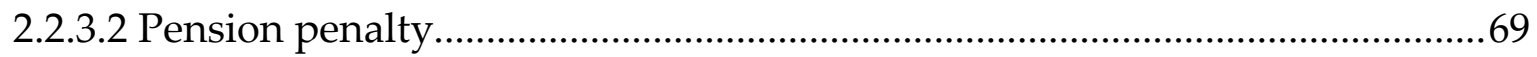

2.2.3.3 Pension bonus ................................................................................................

2.2.4 Combination of work and old-age pension benefits..............................................73

2.3 Conventional early retirement....................................................................................74 


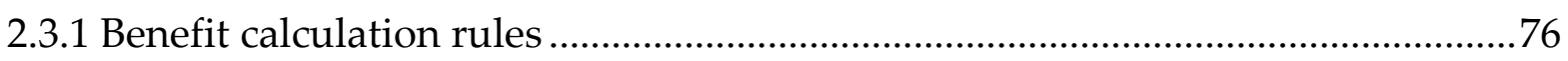

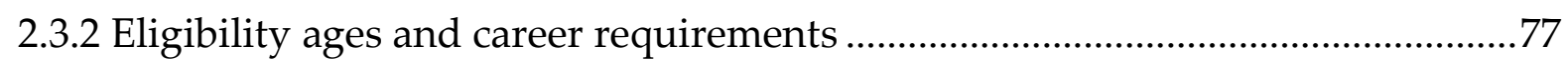

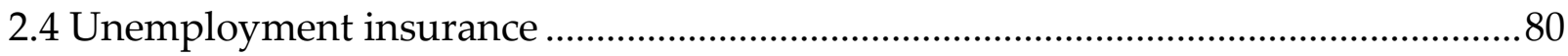

2.4.1 Old-age unemployment .......................................................................................... 81

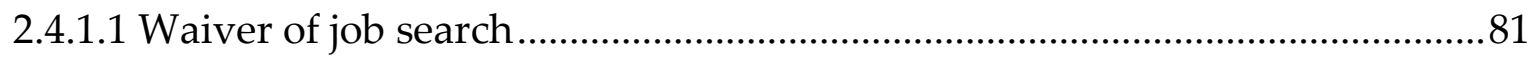

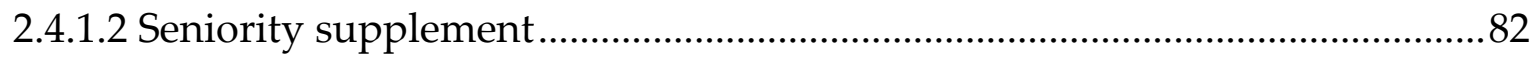

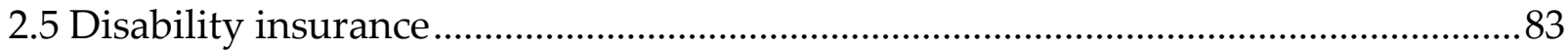

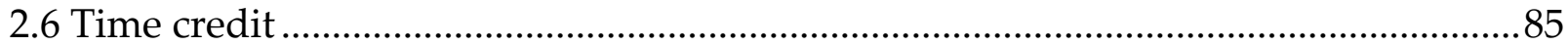

2.7 Income taxes and social security contributions..............................................................86

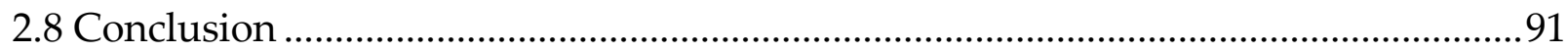

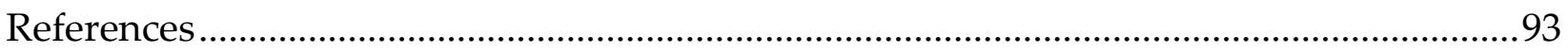

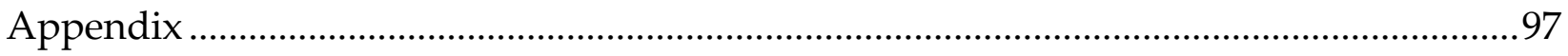

CHAPTER THREE: Social security incentives in Belgium: An analysis of four decades of

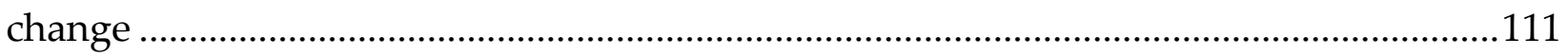

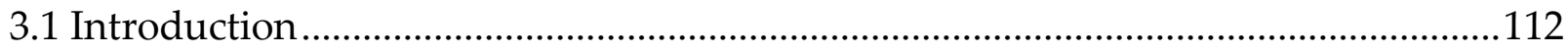

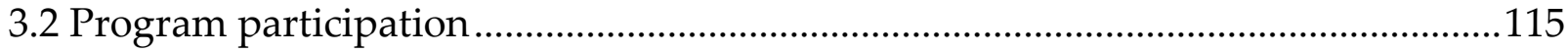

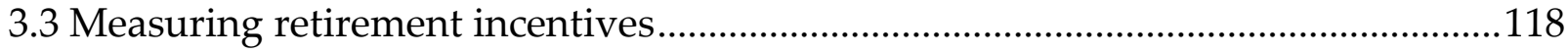

3.3.1 Earning histories ................................................................................................118

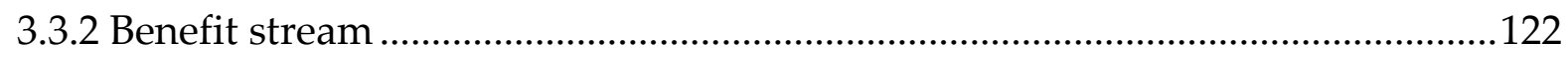

3.3.3 Social security wealth, accrual and implicit tax...................................................123

3.4 Retirement incentives: common profile ...................................................................125

3.4.1 Incentives by pathway ......................................................................................126

3.4.2 Aggregate incentives and employment ...............................................................142

3.5 Retirement incentive: belgian-specific profile ...........................................................151

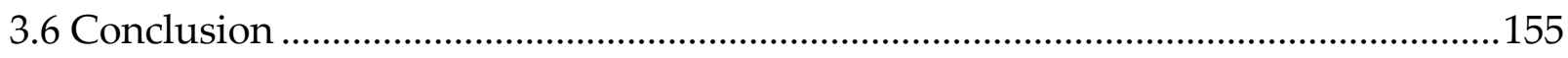

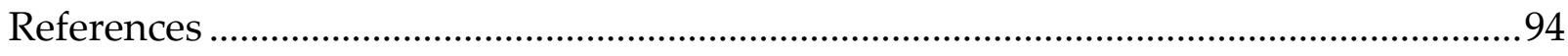


CHAPTER FOUR: Trends in social security incentives

4.1 Introduction

4.2 Incentive measures calculation

4.2.1 Data

4.2.2 Benefits calculation

4.2.3 Incentive measures

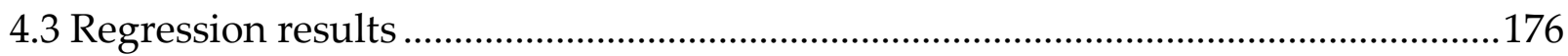

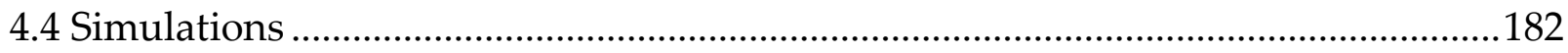

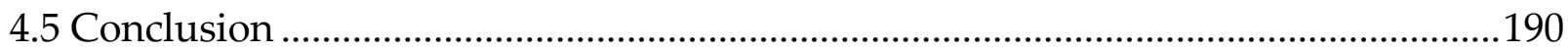

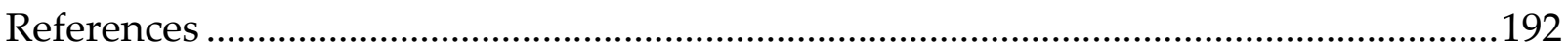

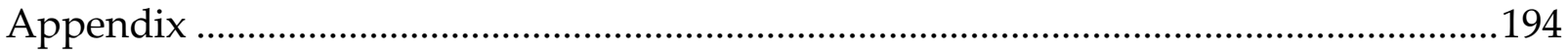

CHAPTER FIVE: Work, labor force exit and benefit claiming patterns in Belgium .......203

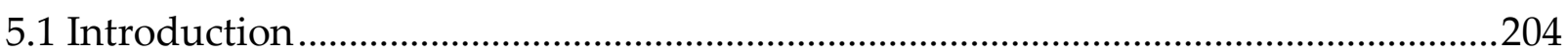

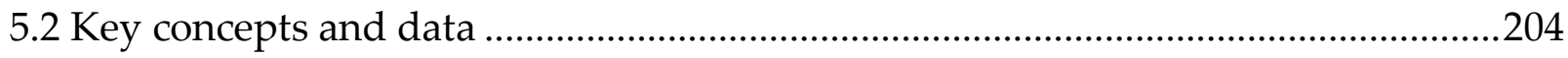

5.2.1 The labor force exit and the benefit claiming decisions ................................208

5.2.2 Data and sample selection ........................................................................212

5.3 Descriptive analysis of employment and retirement behavior............................2214

5.3.1 Employment and benefit claiming …........................................................214

5.3.2 Labor force exit and benefit claiming hazard rates........................................219

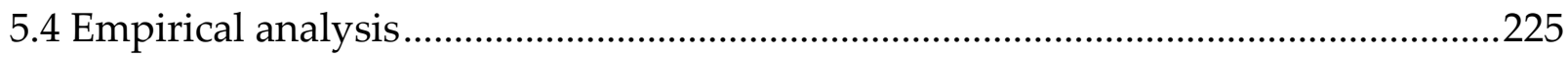

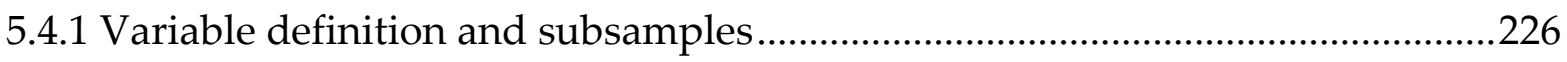

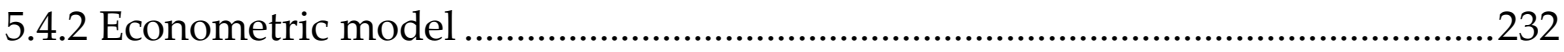

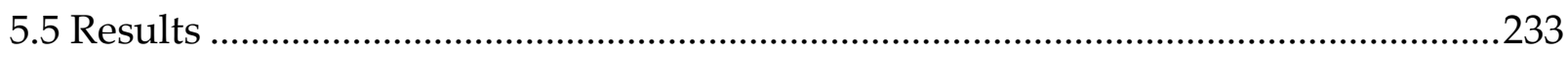

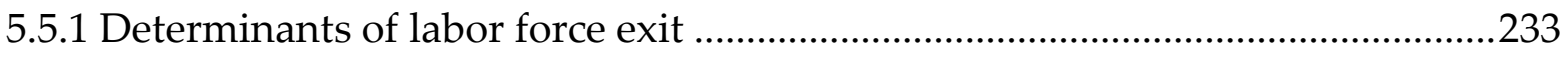

5.5.2 Determinants of benefit claiming ….........................................................2238 
5.6 Conclusion

References

Appendix

.248

CHAPTER SIX: The effect of the household replacement rate on the retirement behavior of individuals in one-earner households .255

6.1 Introduction .256

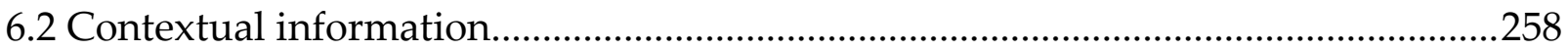

6.2.1 The household replacement rate............................................................................258

6.2.2 Retirement incentives and financially dependent spouse .................................262

6.2.2.1 Spousal characteristics ................................................................................262

6.2.2.2 The influence of the household replacement rate benefits...........................263

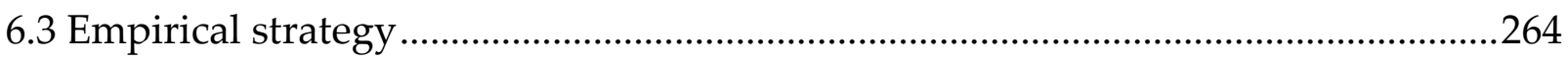

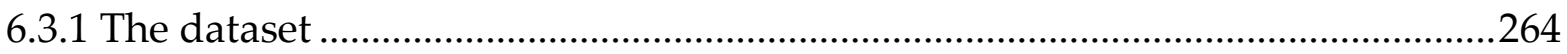

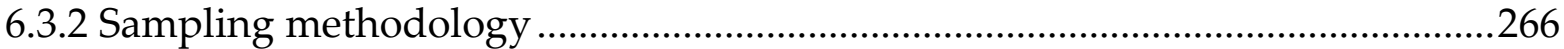

6.3.3 Reconstruction of earnings histories and calculation of pension benefits........267

6.3.4 Validation of old-age pension benefits calculator ..................................................269

6.3.5 Classification into one and two-earner households............................................2.

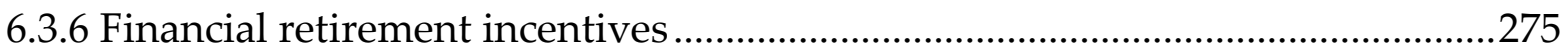

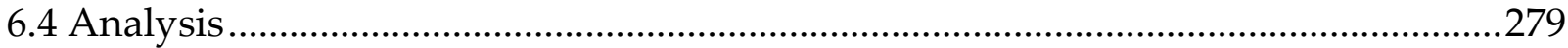

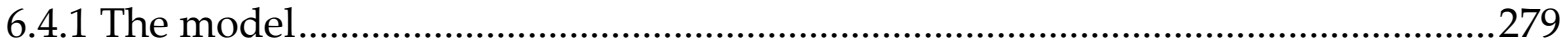

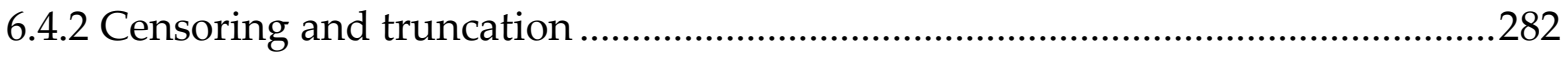

6.4.3 Dependent and explanatory variables .......................................................................28

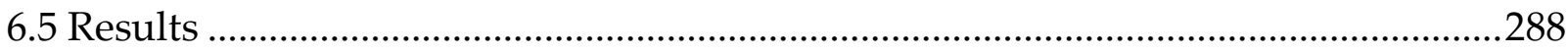

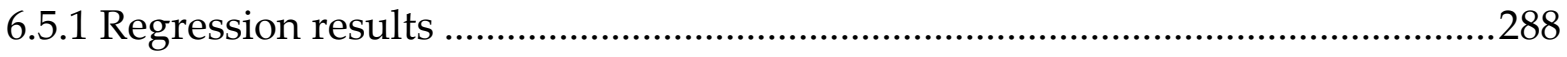

6.5.2 Simulation of a change in the generosity of the household rate benefits .........294

6.5.2.1 Impact on the retirement probability ………..................................................294 
6.5.2.2 Impact on poverty measures

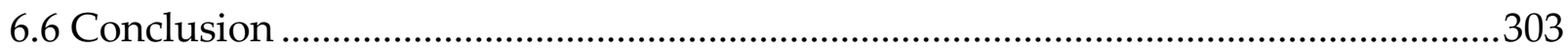

Reference

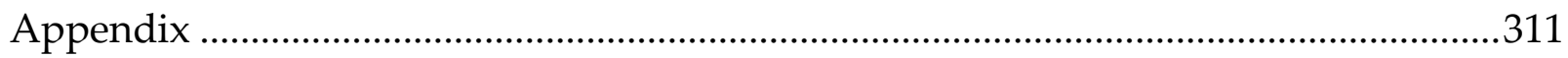

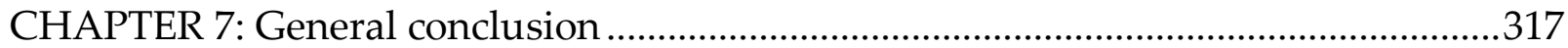




\section{LIST OF TABLES}

Table 2.1: Evolution of the wage floor per year of career (in constant 1996 euros)

Table 2.2: Evolution of early and statutory eligibility ages and career length requirements

Table 2.3: List of royal decrees modifying the minimum pension amount

Table 2.4: Pension bonus benefits amounts - 2014 rules

Table 2.5: Solidarity contribution rate (2020 rules)

Table 2.6: Health and Disability contribution rate (2020 rules)

Table 2.A.1 Evolution of lump-sum wages

Table 2.A.2 Historical evolution of the assimilated wage for periods spent receiving a replacement income

Table 2.A.3 Valorisation coefficients for a person retiring in 2020 (pivotal index 147.31)

Table 2.A.4 Evolution of the pivotal index and add-on factor

Table 2.A.5 Historical evolution of growth coefficients

Table 2.A.6 Evolution of ceilings for pensionable earnings

Table 2.A.7 Differentiated ceilings for assimilated periods for wage-earners

Table 2.A.8 Earnings test for the combination of work and old-age pension receipt, 2020 (pivotal index 147.31)

Table 2.A.9 Ceilings for sickness and disability benefits

Table 2.A.10 Time credit benefits (monthly net amounts)

Table 2.A.11 Historical evolution of the first tax deduction for replacement income

Table 2.A.12 Historical evolution of the second tax deduction for replacement income 
Table 4.1: Main Characteristics of the individuals present in the sample, in their first observation year

Table 4.2: Retirement - Total sample

Table 4.3: Retirement using the probit model - by sex

Table 4.A.3. Regression results - total sample with accrual

Table 4.A.4 Regression results - men with ITAX

Table 4.A.5. Regression results - women with ITAX

Table 5.1: Main characteristics of sampled individuals in the first year of observation

Table 5.2: Mean value of dependent and independent variables by status and sex

Table 5.3: Determinants of labor force exit

Table 5.4: Determinants of benefit claiming

Table 5.A.1: Determinants of labor force exit - correlated random effects probit model

Table 5.A.2: Determinants of benefit claiming -correlated random effects probit model

Table 6.1: Characteristics of sampled individuals

Table 6.2: Main characteristics of sampled individuals by type of household

Table 6.3: Discrete time logistic hazard duration model with random effects - regression results

Table 6.4: Probit model of the probability of being in a one-earner household

Table 6.A.1 Household rate benefits around Europe 


\section{LIST OF FIGURES}

Figure 2.1: Reforms of the old-age pension system in Belgium (1985-2020)

Figure 2.2: Reforms of the old-age unemployment system in Belgium (1985-2020)

Figure 2.3: Reforms of the conventional early retirement system in Belgium (1985-2020)

Figure 2.4: Reforms of the time credit system for older workers in Belgium (2000-2020)

Figure 2.5: Evolution of the CPI, the health index and the pivotal index (1996 - 2019)

Figure 2.6: Evolution of wage floor in current euros

Figure 2.7: Evolution of pensionable earnings ceilings

Figure 2.8: Impact of the pension bonus on old-age pension benefits- 2007 rules

Figure 2.9: Impact of the pension bonus on old-age pension amounts- 2014 rules

Figure 2.10: Historical evolution of career length requirement for the standard conventional early retirement scheme - by eligibility ages and gender

Figure 2.11: Evolution of the first deduction for replacement income

Figure 2.12: Evolution of the second deduction for replacement income

Figure 3.1: Employment rate of older workers (men and women, ages 55-64)

Figure 3.2: Pathways to retirement - men, age 55-59

Figure 3.3: Pathways to retirement - men, age 60-64

Figure 3.4: Pathways to retirement - women, age 55-59

Figure 3.5: Pathways to retirement - women, age 60-64

Figure 3.6: Common earnings profiles

Figure 3.7: Belgian-specific earnings profiles

Figure 3.8: Incentive measures - Old-age pension - men median earners, ages 55-59

Figure 3.9: Incentive measures - Old-age pension - women median earners, ages 55-59

Figure 3.10: Incentive measures - Old-age pension - men median earners, ages 60-64 
Figure 3.11: Incentive measures - Old-age pension - women median earners, ages 60-64 Figure 3.12: Implicit tax of conventional early retirement benefits - men median earners, ages 55-59

Figure 3.13: Implicit tax of conventional early retirement benefits - women median earners, ages 55-59

Figure 3.14: Implicit tax of unemployment benefits - men median earners, ages 55-59

Figure 3.15: Implicit tax of unemployment benefits - women median earners, ages 55-59

Figure 3.16: Implicit tax of disability benefits - men median earners, ages 55-59

Figure 3.17: Implicit tax of disability benefits - women median earners, age 55-59

Figure 3.18: Implicit tax for male median earners by age (55-59)

Figure 3.19: Implicit tax for female median earners by age (55-59)

Figure 3.20: Implicit tax for male median earners by age (60-64)

Figure 3.21: Implicit tax for female median earners by age (60-64)

Figure 3.22: Implicit tax for male median earners by year

Figure 3.23: Implicit tax for female median earners by year

Figure 3.24: Employment rate and ITAX - men, ages 55-59

Figure 3.25: Employment rate and ITAX - women, ages 55-59

Figure 3.26: Employment rate and ITAX - men, ages 60-64

Figure 3.27: Employment rate and ITAX - women, ages 60-64

Figure 3.28: SSW- Old-age pension - ages 55-59 
Figure 4.4: Exit rates by age for men - all sample

Figure 4.5: Exit rates by age for women - all sample

Figure 4.6: Comparison of observed and simulated old-age pension benefits, old-age pension retirees in the sample 2005-2010

Figure 4.7: Average SSW by exit pathway (in 2010 euros)

Figure 4.8: Average aggregated individual SSW, by gender (in 2010 euros)

Figure 4.9: Average ACC, by gender (in 2010 euros)

Figure 4.10: Average ITAX, by gender (in 2010 euros)

Figure 4.11: Retirement rate for men and women- actual and predicted baseline

Figure 4.12: Counterfactual simulation - total population aged 56-65

Figure 4.13: Counterfactual simulation- Men aged 60 and 65 - by year

Figure 4.14: Counterfactual simulation without SEA change- Women - by year

Figure 4.15: Employment survival curve

Figure 4.a.1. Aggregation weights by exit pathways for men (averaged over years)

Figure 4.a.2. Aggregation weights by exit pathways for women (averaged over years)

Figure 5.1: Classification of individuals according to their work and claim status and the labor force exit and benefit claiming decisions

Figure 5.2: Decomposition of employment rates by claiming status and age (men and women, period average 2004-2010)

Figure 5.3: Decomposition of employment rates by claiming status, work intensity and age (men and women, period average 2004-2010)

Figure 5.4: Decomposition of claims in work and claim, by sex and work intensity

Figure 5.5: Labor force exit and benefit claiming hazard rates by age and sex (period average $2004-2010$ )

Figure 5.6: Decomposition of labor force exit hazard rates by claiming status, age and sex (period average $2004-2010$ )

Figure 5.7: Decomposition of benefit claiming hazard rates by working status, age and sex (period average $2004-2010$ ) 
Figure 5.8: Predicted labor force exit probability by claiming status, part-time work and sex

Figure 5.9: Predicted labor force exit probabilities of workers in the group work and claim by types of claim and sex

Figure 6.2: Evolution of pension benefits by replacement rate, marital status and gender (1992-2019)

Figure 6.3: Proportion of pension by amount, replacement rate and gender (average 2002-2019)

Figure 6.4: Evolution of estimated old-age pension amounts by age and gender (2017 constant euros)

Figure 6.5: Estimated and observed old-age pension benefits by gender

Figure 6.6: Retirement hazard rates by age and type of household

Figure 6.7: Evolution of social security wealth by age

Figure 6.8: Evolution of accruals by age

Figure 6.9: Illustration of standard observation, left truncation and right censoring in the data

Figure 6.10: Retirement hazard rates for workers in one-earner households - baseline (75\% replacement rate) and suppression of household replacement rate $(60 \%$ replacement rate)

Figure 6.11: Change in retirement probability for individuals in one-earner households for different reform scenario of the household replacement rate

Figure 6.12: Dispersion of equivalized old-age pension benefits by type of household

Figure 6.13: Average equivalized old-age pension benefits by type of households under different reform scenarios of the household replacement rate

Figure 6.14: Headcount ratio and average poverty gap of one-earner households under different household replacement rate reform scenarios

Figure 6.A.2 Evolution of monthly gross salary by age (average 1999 - 2017) 


\title{
GENERAL INTRODUCTION
}

\author{
Anne-Lore Fraikin
}




\subsection{BACKGROUND AND MOTIVATION}

Subsequent to population ageing, numerous European countries are facing a serious threat to the financial sustainability of their pension systems. On top of factors influencing old age spending (e.g. increases in life expectancy ${ }^{1}$ and the massive arrival of baby boomers at the age of retirement) other factors affect the financing capacity of these countries, such as decreases in fertility and migration rates. In Belgium, it is predicted that gross public pension expenditure will increase by 6.6 percentage points of GDP between 2016 and 2070 following an increase of the old-age dependency ratio from 28.4 to 45.2 percent during the same period of time (Federal Planning Bureau, 2017).

In addition to these demographic trends, Belgium must cope with relatively low elderly employment rates and one of the earliest average effective retirement ages in the OECD. Indeed, though up from the record lows of 58.3 (men) and 56.4 (women) in the mid-1990's, Belgium still underperforms as compared to other OECD countries with an average retirement age for men of 61.6 and 60.5 for women in 2018 (OECD, (2017,2019)). Nonetheless, concurrently to a trend observed in many developed countries, the country has witnessed a major transformation of its labor market environment over the last decades (see figure 1). The employment rate of elderly² Belgian men exhibits a u-shaped curve - with a decline in the 1980's and 1990's and then an upward trend starting in the 2000 's. The employment rate of elderly Belgian women is characterized by a long-term upward trend, reflecting a structural increase in the labor force participation of women.

\footnotetext{
${ }^{1}$ Life expectancy at age 65 is predicted to increase by 5 years for both men and women between 2016 and 2070. Consequently, the duration of retirement is predicted to increase from 20.8 (24.4) years in 2017 to 24.3 (27.5) years in 2070 for men (women) (Federal Planning Bureau, 2017).

2 Unless indicated otherwise, we refer to elderly as individuals aged more than 54 . Since, the majority of early labor force exit pathways open above age 54 and because the vast majority of individuals retire at age 65 (the statutory eligibility age in most cases), we refer to the elderly working population as working individuals aged between 55 and 64 .
} 
The Federal Planning Bureau (2017) forecasts that these trends will endure and predicts an increase in elderly labor force participation from 45.5 in 2016 to 62.4 in 2070 and an associated increase in total contributory period from 39.2 (34.2) years in 2017 to 40.6 (39.0) years in 2070 for men (women).

Figure 1: Employment rate of elderly Belgian workers (ages 55-64)
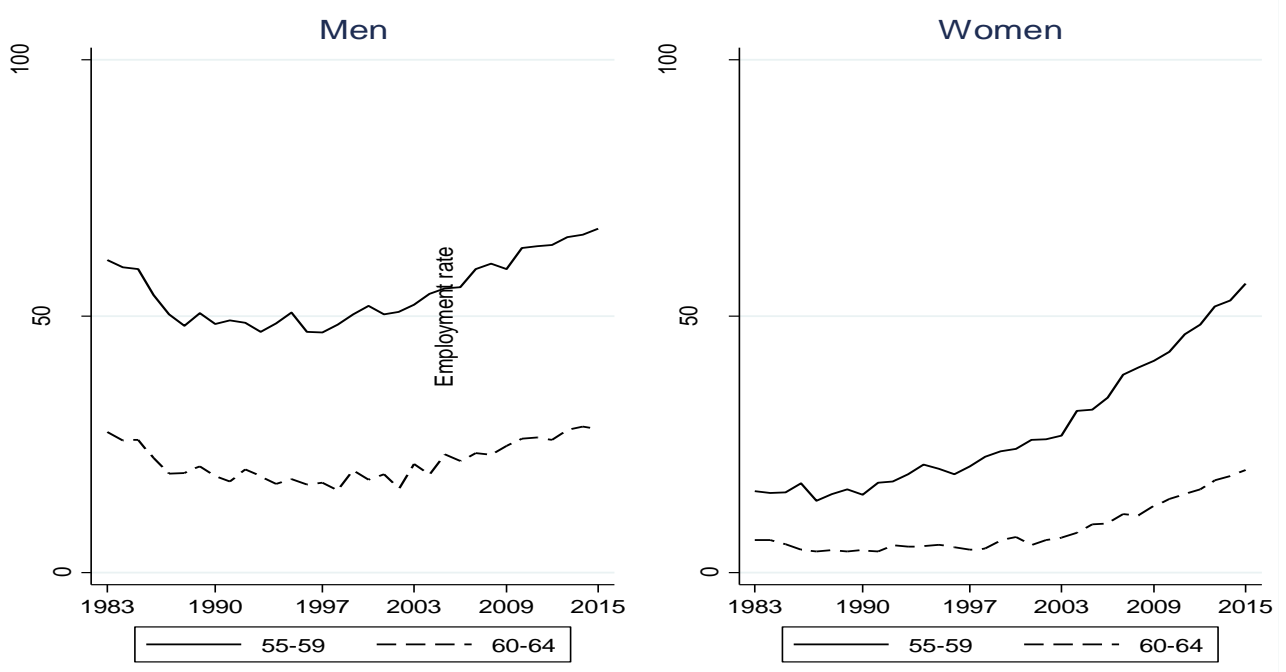

Source: Eurostat (2017)

While these increases in employment rates show potential for improving the financial sustainability of the pension system, these tendencies are in no way sufficient to absorb the overwhelming burden of population ageing on public old age spending. Indeed, the evolution of the coverage ratio, the benefit ratio and the increase in elderly employment rates are predicted to decrease gross public pension expenditure by 2.1, 0.5 and 0.9 percentage points, respectively (Federal Planning Bureau, 2017). Assuming unchanged policy, the contribution rate of workers would have to increase twofold by 2070 to safeguard the current level of old-age pension benefits (Hindriks, 2015).

Confronted with predictable demographic trends, the Belgian government has implemented various social security reforms since the 1990's to restrict the impact of 
population ageing on the financial sustainability of the country's pension system. Ultimately, the necessary reforms boil down to a combination of three measures: increased contributions or tax financing, lowered benefits and increases in the effective retirement age. Retirement reforms in Belgium have mostly been directed at the last option, i.e. increase in the effective retirement age, and are divided into two main streams. On the one hand, access to early labor force exit routes outside of the old-age pension system has been tightened. On the other hand, efforts have been made to reduce the incentives of early old-age pension benefits claiming.

Limiting early labor force exit pathways might not be sufficient to increase workers' effective retirement age. First, it is essential that older workers are encouraged to stay on the labor force. Some authors ${ }^{3}$ mention that allowing for phased-in retirement, in which a worker can gradually decrease his working hours and/or receive social security benefits, is one of the solutions to increase the average effective retirement age. Second, from a labor demand point of view, companies should be incentivized to keep their older workers longer. However, negative beliefs about decreases in productivity and the high price of older workers, limit the practicality of this second option.

On top of addressing financial sustainability concerns, pension adequacy ${ }^{4}$ reforms remain an important societal challenge for many years to come (Holzmann and Hinz, 2005). Indeed, the Pension Reform Committee (2014) finds an elderly monetary poverty incidence rate ${ }^{5}$ of 19.4 percent of the elderly population $(65+)$, which is relatively higher than most neighboring countries. Nonetheless, were house ownership included in the

\footnotetext{
${ }^{3}$ See Benitez-Silva and Heiland (2008), Börsch-Supan et. al. (2017) and Duval (2003), among others.

${ }^{4}$ An adequate pension system provides a retirement income that prevents from old-age poverty and allows for consumption smoothing at older ages (Holzmann and Hinz, 2005).

${ }^{5}$ Using a poverty line equal to 60 percent of the median equivalized disposable income of the whole population.
} 
old-age pension calculations, the incidence rate would be brought down to 13 percent (Pension Reform Committee (2014), Hindriks (2015)). Moreover, the elderly poverty intensity ${ }^{6}$ rate is relatively low in Belgium because many older individuals receive an income that is right below the poverty line. Overall, the Ageing Study Committee (2020) reports that the Belgian elderly poverty and inequality rates have been decreasing uninterruptedly over the period 2005-2014 and have now stabilized to a level close to that of the whole population. Intensified women activity rates and the continuous adaptation of social and minimum pensions to increases in well-being are among the factors at the root of such an increase. In spite of these improvements, income inequality at older ages persists between sexes, socio-economic status and household arrangements and there is still a substantial need for reforms to achieve an adequate level of pension adequacy.

\subsection{THE RETIREMENT BEHAVIOR AND THE SOCIAL SECURITY SYSTEM}

Following declines in elderly labor force participation and the ageing of baby boomers, research on the topic of the economics of retirement has grown considerably in recent years. In this portion of the literature, the majority of studies explore the determinants of the retirement decision, which is multi-faceted and is influenced by factors in both the microenvironment (e.g. personal and household characteristics, partner characteristics, income and wealth, leisure opportunities, etc.) and in the macroeconomic environment (e.g. job opportunities, institutional factors, etc.) (Coile, 2015).

The concomitance of the trends in elderly labor force participation and the wave of reforms that tightened the coverage and generosity of the social security system has spawned a vast literature on the link between the retirement decision and financial

${ }^{6}$ Poverty intensity is measured as the distance between the poor's earnings and the poverty line. 
retirement incentives stemming from the social security system. Coile (2015) mentions that these studies mainly differentiate between two effects: an (i) income and a (ii) substitution effect.

On the one hand, the design of old-age pension systems may generate an income effect if the benefits received are greater (lesser) than the contributions paid in. In particular, intracohort redistribution through the minimum pension, pensionable earnings ceilings and floors and the household replacement rate may generate such an income effect and can potentially influence the retirement decision. ${ }^{7}$

On the other hand, a substitution effect is generated if an additional year of work decreases (increases) the amount of net pension wealth, leading to earlier (later) retirement. Indeed, an additional year of work can potentially have several distinct effects on a worker's expected pension wealth. First, the extra income earned will be incorporated in the pension benefits calculation and thus increases one's expected pension wealth. Second, since life expectancy is assumed fixed, a worker will forgo the receipt of pension benefits for one year, which decreases his total expected pension wealth. Third, actuarial adjustment programs that reward late retirement (or punish early retirement) also lead to an increase (decrease) the worker's expected pension wealth. The net effect of these factors determines if working for one extra year embodies a positive or a negative accrual of the worker's expected pension wealth.

Many studies have examined these two effects and the overall impact of the financial retirement incentives created by the social security system on the retirement behavior of older workers. The purpose of these studies is often to model the role that social security

\footnotetext{
${ }^{7}$ Inter-cohort redistribution in pay-as-you-go pension systems also generates an income effect since early pension beneficiaries received benefits without having contributed to the system.
} 
benefits play in determining the retirement decision through reconstructing hypothetical retirement incentives measures using empirical data and studying their implications in determining retirement. While the modelling techniques differ greatly, the conclusions of these authors are generally similar: they find that social security rules have a large effect on the retirement decision but that it cannot explain the full breadth of the evolution of elderly labor force participation over time (see Coile and Gruber, 2001). In particular, Stock and Wise (1990) find that large penalties on additional work exist in Belgium, France, Italy and the Netherlands. Gruber and Wise (1999) are among the first to provide a large-scale study of financial retirement incentives across a panel of countries and also observe large penalties on additional work once social security benefits become accessible for older workers. Gruber and Wise (2004) follow up on their previous work and use an option-value framework developed by Stock and Wise (1990) to study the empirical link between individual financial retirement incentives and retirement behavior. They identify a strong correlation between dynamic retirement incentives and labor market behavior, indicating that higher marginal returns to work lead to later retirement. Moreover, they predict the effect of illustrative reforms in a set of developed countries (including Belgium) and demonstrate that changes in social security provisions would have a highly significant effect on the labor force participation of older workers.

In Belgium, previous studies have pointed at the important role of social security incentives and institutional changes in explaining the labor force participation of the elderly. Dellis et. al. (2004) use administrative data over the period 1993-1995 to compute financial retirement incentives and estimate the impact of several old-age pension statutory eligibility age reforms. They note that most Belgian workers face a penalty on continued work at older ages, sometimes as early as 58 , and they find empirical evidence of the link between these penalties and the retirement behavior. Desmet et. al. (2007) use a microsimulation model and analyse the budgetary and behavioral impact of another set 
of social security reforms. They find that the various early labor force exit programs create substantial work disincentives at older ages. Jousten and Lefebvre (2013) use SHARE survey data and an option-value framework to test the effect of two reform scenarios: an increase in eligibility ages and an increase in early exit rules. They find a significant impact of financial incentives, health and education variables on retirement probability. Jousten and Tarantchenko (2014) study the link between the retirement decision and financial retirement incentives using an administrative dataset from the Crossroad Bank of Social Security and put emphasis on the analysis of an indicator of the social security eligibility status. They observe that the eligibility status indicator has a higher effect on the retirement probability compared to financial retirement incentives. Jousten and Lefebvre (2016) use an option-value framework to study the role of health and financial retirement incentives on the retirement decision and find that both variables play a prominent role in the retirement decision. Jousten and Lefebvre (2019) use an option value model to study the retirement incentives faced by singles and couples and find that retirement incentives differ greatly between men and women. Moreover, they find that augmented financial retirement incentives measures, which allow for differences between singles and couples, are particularly relevant in a country like Belgium, with strong dependent spouse benefits components.

These studies generally corroborate the international literature and attest of a positive income effect - i.e. the higher the pension wealth, the earlier the retirement - and a negative substitution effect - i.e. the higher marginal returns to work, the later retirement. Belgium is a particularly interesting country case study. First, it has an advanced and complex set of early labor force exit pathways. Second, the government has implemented a large number of reforms to its social security programs in recent years, which thus presents a rich background for the study of reform proposals. Finally, given the relatively low elderly employment rate and low effective retirement age, the country presents an 
outstanding background to explore the link between the retirement behavior and financial retirement incentives.

\subsection{THESIS OUTLINE}

The objective of the present thesis is to answer the question: "How is the retirement behavior of older workers influenced by the social security system?". In other words, we explore the impact of the social security system's provisions as a driver of the retirement behavior of older workers, using Belgium as a case study. We aim our attention at the evolution of social security benefit calculation and eligibility rules and their role in determining financial incentives for retirement. Following rapid population ageing and the influx of baby boomers at the age of retirement, the financial sustainability of many old-age pension systems is threatened and it has become crucial that governments have a clear idea of how their policies influence the retirement decision. In this section, we present a brief overview of the chapters.

The first chapter of the thesis comprises the general introduction. Chapter seven summarizes the key findings of the present work, reviews some limitations of our analysis, and indicates directions for further research.

The second chapter of the thesis contains a thorough review of the Belgian social security landscape relevant for the study of retirement. In particular, we list the various benefit calculation and eligibility reforms that have occurred in the country since the 1980s in the four main (early) labor force exit pathways for older workers: the old-age pension program, the conventional early retirement program, the unemployment insurance program, the disability insurance program, and we also examine the time credit program. We illustrate the complexity and sometimes incoherency of the Belgian social security 
system, which generates an enabling environment for intertwined effects between programs and reforms. As a matter of fact, such a convoluted system sometimes leads to specific social security reforms reverberating on the effects of other reforms and even on the take-up of other programs and even on other reforms. The lack of careful long-term planning and cautious ex-ante and ex-post analysis of the effect of social security reforms worsen the situation even further. The analysis presented in this chapter is relevant for policy makers and researchers alike. On the one hand, it is crucial that decision makers have a good understanding of the current system as well as its evolution over time in order for them to pave the way for the necessary sustainability reforms that lie ahead. On the other hand, some trivial benefit calculation parameters are often discarded when computing financial retirement incentives for the analysis of the retirement behavior. Some of these parameters (e.g. evolution of taxation rules) are nonetheless of prime significance and have an important role to play in the computation of financial retirement incentives.

The third chapter presents a microsimulation model aimed at assessing the evolution of financial retirement incentives stemming from the social security system from the 1980s to the present day for different subgroups of the population. Indeed, the respective importance of individual incentives and institutional changes in explaining observed labor supply and retirement patterns over the last decades remains unclear. We update the work of Pestieau and Stijns (1999) by using recent data and we calculate the financial incentives to exit employment for various typical workers, differentiated by age, year, income level, marital status and sex - integrating changes both in benefits and in the tax system. We compute financial retirement incentives that include not only instantaneous income but also the complete set of survival and eligibility contingent benefits streams, forecast all the way until the end of life. Such expected benefits streams are influenced by the exposure and eligibility of an individual to social security programs that work as early 
labor force exit pathways. The model allows us to separate age and year effects, incidentally also permitting us to take phased reforms and grandfathering provisions into account. The model also permits the study of an array of scenarios in terms of earnings level, earnings growth, mortality, as well as a simulation of modified system parameters. We find that the tightening of eligibility conditions and the greater variation in the generosity of some benefits translate into important changes of our incentive measures to retire. Specifically, we find that workers above the age of 60 typically face an implicit tax on additional work, indicating a retirement incentive created by the social security system. At the macroeconomic level, the chapter concludes that retirement incentives from the social security system are moderately correlated with the employment rates of older Belgian workers. In conclusion, in this chapter, we provide an analysis of past financial retirement incentives, which can serve as evidence that the social security system may well have an important impact on the retirement decision and that careful ex-ante policy analysis should be realized before any significant old-age pension sustainability reform.

The aim of the fourth chapter is to examine the empirical link between the financial retirement incentives created by the social security system and the retirement decisions of older Belgian workers. We update and expand the work of Dellis. et. al. (2004) in terms of period coverage by using a more recent panel administrative dataset and we provide additional simulations of reforms. From each worker's earning history, we compute their expected old-age pension benefits and various financial incentive measures. We test the correlation between the retirement decision and our financial retirement incentive variables using various econometric models and specifications. Our general regression analysis shows a positive income effect (i.e. more generous measures induce higher retirement rates) and a negative substitution effect (i.e. higher returns to work lead to lower retirement rates). When considering men and women separately, we find a stronger 
effect of our financial retirement incentive variables on the retirement probability of women. Finally, based on these regression results, we perform a series of simulations in which we neutralize the effect of some of the social security reforms that occurred in the country during our observation period. We find that the increase in the statutory eligibility age of women did indeed lead to an increase in the incentives to stay on the labor market. However, we find that the pension bonus did not have the expected effect of keeping workers on the labor market longer, but instead had the opposite effect by generating an increase in the income effect of financial incentives on retirement. In this chapter, we show that some of the reforms did not have the desired effect on retirement probability, and we demonstrate once again the relevance of careful ex-ante impact analysis of social security reforms and the role they can play in improving the financial sustainability of the pension system.

The fifth chapter documents the dissociation of the retirement decision into a labor market exit and a benefit claiming decision. In the literature and in the public debate, the decision to retire is frequently equalled to stop working and simultaneously claim (early) retirement benefits for the first time. Increasingly, however, individuals face more complex choice sets, that sometimes lead them to - optimally or not - disjoin these two decisions. We consider four different sub-statuses of individuals, based on work and benefit claiming statuses. Such a decomposition matters not only for the analysis of the retirement behavior but also from the public finance and the pension system sustainability points of view. First, social security reforms have the potential to influence both decisions differently - possibly disjoining them. Indeed, we study the determinants of both decisions separately and find that they are influenced by different factors. Second, the option of combining (part-time) work with social security benefits claiming influences labor market decisions at older ages, arguably encouraging some workers to stay in the labor force longer. In fact, we study the implications of this decomposition on the 
evolution of macro level employment trends and find that the recent increase in elderly labor force participation was mostly caused by a rise in part-time work and in the prevalence of workers who are claiming benefits. Moreover, we show that access to social security benefits (such as time credit, for example) does not have the desired effect of keeping older workers on the labor force longer as these individuals have a higher tendency to retire early. Finally, in Belgium, the complex landscape of labor force exit and benefit claiming options, the ubiquitous nature of partial or full labor force exit options and the absence of penalty for early claimers of old-age pension benefits renders the short and the long-term fiscal stakes particularly high. In conclusion, we show that such a decomposition of employment and retirement trends would prove to be highly beneficial for both research and policy analyses concerns and in easing the progress towards a more sustainable pension system.

The objective of the sixth chapter is to examine the retirement behavior of individuals in one-earner households, i.e. households composed of one earner and one partner who is financially dependent. These individuals are automatically granted a more generous replacement rate in the calculation of their old-age pension benefits, the so-called household replacement rate. In fact, the Belgian Pension Reform Committee recently recommended that the household replacement rate be removed for every new pensioner, except for those receiving the minimum pension. We provide an ex-ante impact evaluation of the removal of the household replacement rate on both pension sustainability and pension adequacy measures. Specifically, we test whether the household replacement rate entails a work (dis)incentive mechanism promoting (harming) pension sustainability and furthermore, we analyse the role of the household replacement rate in ensuring pension adequacy from a public policy perspective. We use the survey dataset SHARE and a discrete time logistic duration model to study the link between the retirement decision and our financial retirement incentive measures. We find 
a positive and significant income effect, a negative and significant substitution effect, and a significant effect of age and social security eligibility rules on the retirement probability. We find no additional impact of being in a one-earner household on top of the indirect impact through the financial retirement incentives. Finally, we simulate the impact of several changes in the household replacement rate generosity on the retirement probability and on poverty measures of individuals in one-earner households. We find an important effect of the household rate on poverty measures and a rather limited negative impact of the program on the retirement probability through its income effect. However, since households with asymmetrical working arrangements are often at the lowest part of the income distribution, the substantial effect of the household replacement rate on poverty measures is a motive to use a mechanism such as a poverty alleviation tool. In view of these results, we advocate in favour of the recommendation of the Pension Reform Committee to remove the household replacement rate for every pensioner except for those in the lowest income deciles. Indeed, income redistribution among the elderly need not be targeted at a specific type of household and policies such as pensionable earning minima, minimum pension benefits and the inclusion of replacement income periods in the pension benefits calculation effectively serve the income redistribution goal and do not favour a certain type of household over another. In this chapter, we take a step further from past chapters and present an ex-ante policy reform analysis on both pension sustainability and adequacy measures to provide evidence of the relevance of these analyses for old-age pension sustainability reforms. 


\section{REFERENCES}

Ageing Study Committee. (2020). Rapport annuel 2020. Bruxelles: Conseil supérieur des finances.

Benitez-Silva, H., and Heiland, F. (2008). Early claiming of social security and labor supply behavior of older Americans. Applied Economics, 40(23), 2969-2985.

Börsch-Supan, A., Bucher-Koenen, T., Kutlu-Koc, V., and Goll, N. (2017). Dangerous Flexibility - Retirement Reforms Reconsidered. MEA DIscussion Papers.

Coile, C., and Gruber, J. (2001). Social Security Incentives for Retirement. In D. Wise (Ed.), Themes in the Economics of Aging. Chicago: University of Chicago Press.

Coile, C. (2015). Economic determinants of workers' retirement decisions. Journal of Economic Surveys, 29(4), 830-853.

Dellis, A., Desmet, R., Jousten, A., and Perelman, S. (2004). Analyse de la cessation d'activité en Belgique. Reoue française d'économie, 18(1), 99-132.

Desmet, R., Jousten, A., Perelman, S., and Pestieau, P. (2007). Microsimulation of social security reforms in Belgium. In J. Gruber, and D. Wise, Social Security Programs and Retirement around the World (pp. 43-82). Chicago: University of Chicago Press.

Duval, R. (2003). The retirement effects of old-age pension and early retirement schemes in OECD countries. Economics Department Working Papers(370).

Eurostat, (2017). European Labor Force Survey, European Union, Luxembourg.

Federal Planning Bureau. (2017). Economic Policy Committee's Ageing - Belgium:

Country Fiche 2017. Bruxelles: Federal Planning Bureau.

Gruber, J., and Wise, D. (1999). Social Security and Retirement around the World. Chicago: University of Chicago Press.

Gruber, J., and Wise, D. (2004). Social Security and Retirement around the World:

Microestimation. Chicago: University of Chicago Press. 
Hindriks, J. (2015). Quel avenir pour nos pensions? Les grand défis de la réforme des pensions. Bruxelles: Pierre de Boeck.

Holzmann, R., and Hinz, R. (2005). Old Age Income Support in the 21st Century: An international perspective on pension systems and Reform. Washington D.C.: The World Bank.

Jousten, A., and Lefebvre, M. (2013). Retirement incentives in Belgium: Estimations and simulations using SHARE data. De Economist, 161 (3), 253-276.

Jousten, A., and Lefebvre, M. (2016). Health Status, Disability and Retirement Incentives in Belgium. In D. Wise, Social Security Programs and Retirement around the World: Disability Insurance Programs and Retirement (pp. 179-210). Chicago: University of Chicago Press.

Jousten, A., and Lefebvre, M. (2019). Spousal and Survivor Benefits in Option Value Models of Retirement: An Application to Belgium. Journal of Pension Economics and Finance, 66-87.

Jousten, A., and Tarantchenko, E. (2014). New evidence on the social security incentives as drivers of retirement behavior. SSRN Electronic Journal. 10.2139/ssrn.2429287.

OECD. (2017). Pensions at a glance 2017: country profiles - Belgium. Paris: OECD. OECD. (2019). Pensions at a Glance 2019. Paris: OECD.

Pension Reform Committee 2020-2040. (2014). Un Contrat Social Performant et Fiable. Bruxelles: SPF Sécurité Sociale.

Pestieau, P., and Stijns, J. (1999). Social Security and Retirement in Belgium. In J. Gruber, and D. Wise, Social Security and Retirement around the World (pp. 37-71). Chicago : University of Chicago Press.

Stock, J., and Wise, D. (1990). Pensions, the option value of work, and retirement. Econometrica, 58, 1151-1180. 
CHAPTER TWO

\section{HISTORICAL EVOLUTION OF THE BELGIAN SOCIAL SECURITY LANDSCAPE FOR OLDER WORKERS}

Anne-Lore Fraikin 


\subsection{INTRODUCTION}

The Belgian social security system is organized in three regimes: one for wage earners, one for civil servants and one for self-employed workers. In this thesis, the focus is set on the wage-earner regime that has not only the largest enrolment ${ }^{8}$ but also represents the largest budgetary item in public old age spending. The scheme for civil servants (resp. self-employed) is characterized by higher (resp. lower) average benefits and very distinct contribution and benefits calculation rules. Besides, the financing of the three regimes differs substantially, for civil servants (resp. self-employed) all (resp. a more substantial share) of budgetary funds stem from general government revenues.

Before we study the role that the social security system has played on the retirement behavior of older Belgian workers, we describe the main reforms of the four retirement programs available to wage earners since the eighties: the old-age pension, the conventional early retirement, the unemployment insurance and the disability insurance schemes. ${ }^{9}$ Additionally, we take a closer look at the system of time credits for older workers. In figures 2.1 to 2.4, we summarize the major social security reforms in Belgium since the mid-1980's in a timeline format.

This chapter is structured as follows. Section 2.2 describes the old-age pension system reforms over the past decades. Section 2.3, 2.4, 2.5 and 2.6 describe the conventional early retirement, the unemployment insurance, the disability insurance and the time credit programs, respectively. Section 2.8 concludes.

\footnotetext{
8 In 2019, there were 2,043,902 beneficiaries in the wage-earners regime, 561,954 beneficiaries in the selfemployed regime and 250,094 beneficiaries in the civil servants regime (National Pension Office, 2019). ${ }^{9}$ In addition, social assistance benefits and social pensions, as well as workplace accident and professional disease benefits exist - but play a less prominent role in the analysis of retirement behavior.
} 
Figure 2.1: Reforms of the old-age pension system in Belgium (1985-2020)

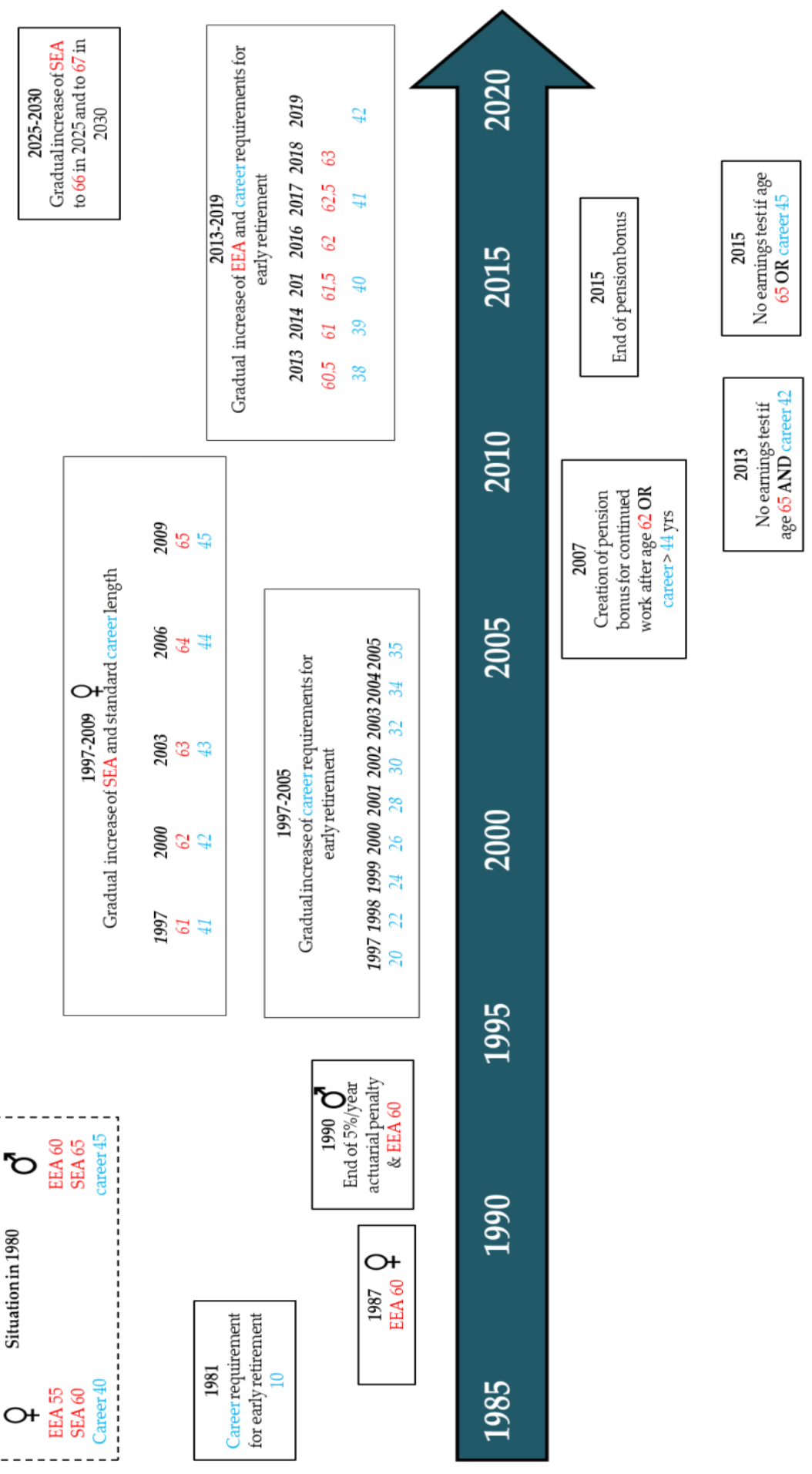

Note: EEA: Early eligibility age, SEA: statutory eligibility age.

Age eligibility conditions are in red, career length requirements are in blue.

Source: See reference list 
Figure 2.2: Reforms of the old-age unemployment system in Belgium (1985-2020)

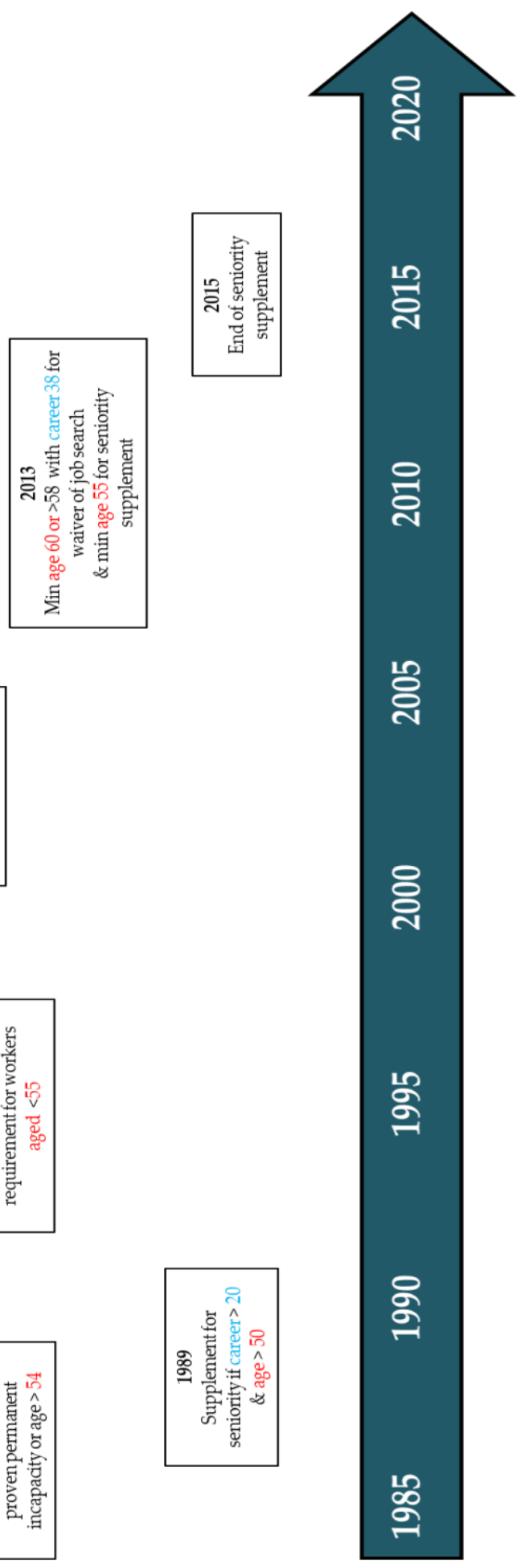

Note: Age eligibility conditions are in red, career length requirements are in blue.

Source: See reference list 
Figure 2.3: Reforms of the conventional early retirement system in Belgium (1985-2020)

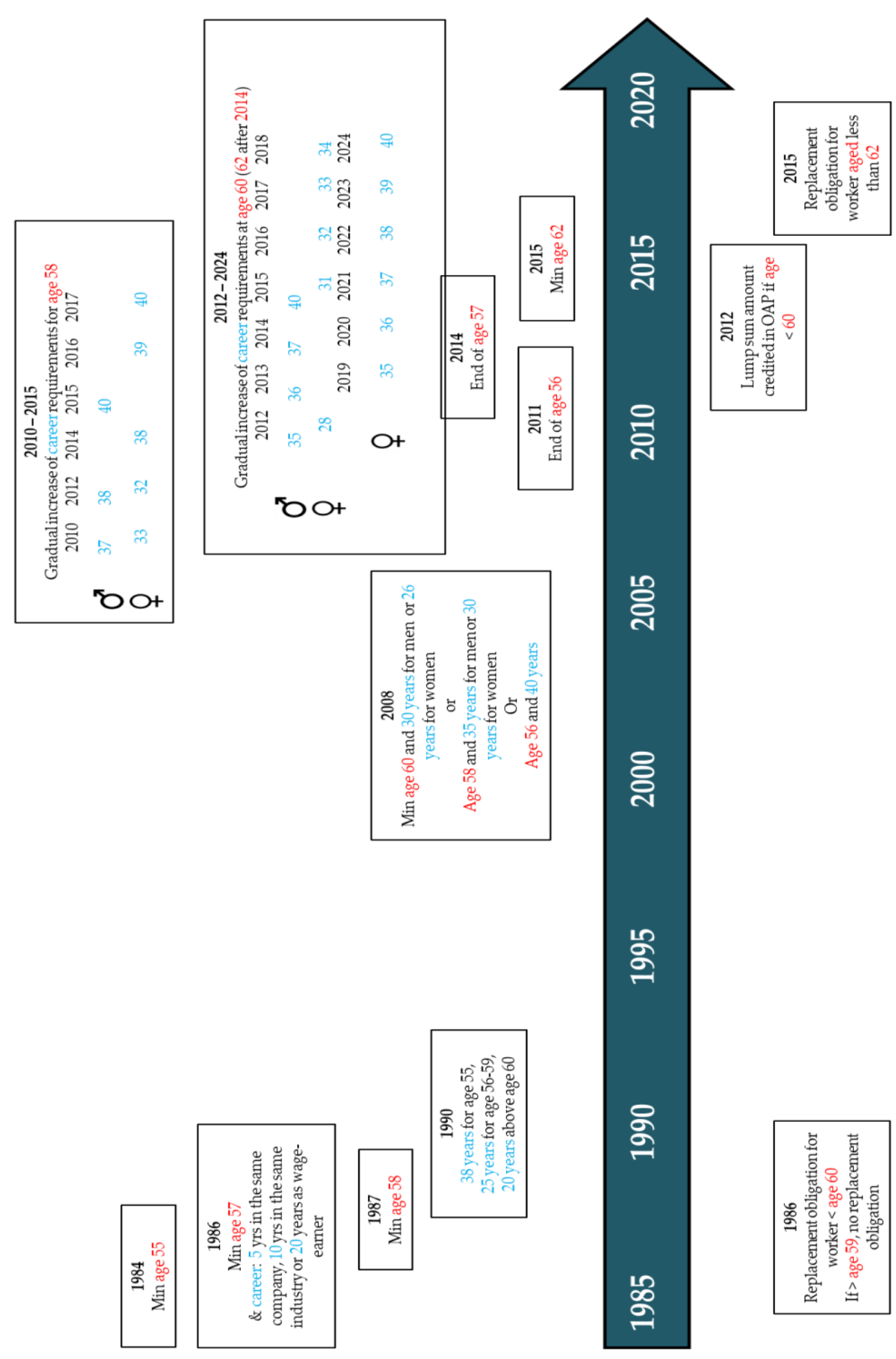

Note: Age eligibility conditions are in red, career length requirements are in blue.

Source: See reference list 
Figure 2.4: Reforms of the time credit system for older workers in Belgium (2000-2020)

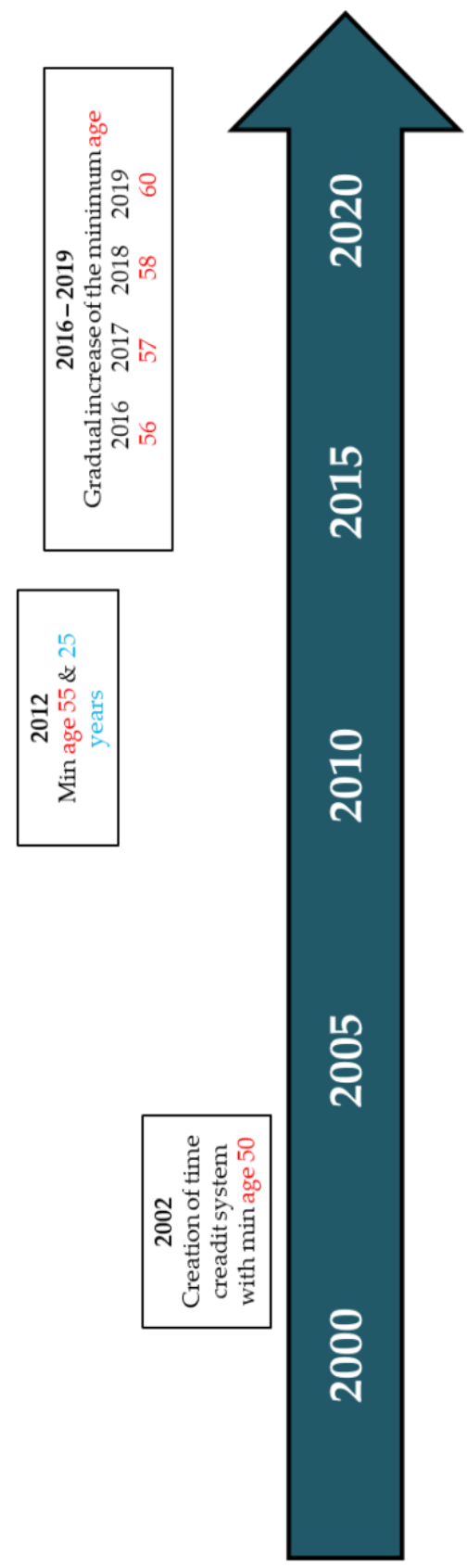

Note: Age eligibility conditions are in red, career length requirements are in blue.

Source: See reference list 


\subsection{OLD-AGE PENSION}

The old-age pension system for wage earners consists of three pillars: the first one is a public pension system ${ }^{10}$, the second one is an occupational pension system and the third one involves voluntary private retirement savings. ${ }^{11}$ The main configuration of the public pension system was established after the Second World War under stable economic growth and encouraging demographic trends. At first, several schemes for various wageearners workers co-existed and the system was a mix of defined contributions and defined benefits. In $1967^{12}$, the wage-earner old-age pension regime is created with the aim of establishing a system of solidarity between every wage-earner worker: blue and whitecollar workers in the private sector ${ }^{13}$, miners and marine workers. ${ }^{14}$ The system became a fully defined benefits system in 1955 for blue-collar workers and in 1957 for white-collar workers. ${ }^{15}$ Nowadays, the public pension system is essentially a mandatory pay-as-yougo scheme that is financed by tax-deductible employer and employee contributions as well as public transfers. ${ }^{16}$

\footnotetext{
${ }_{10}$ Unless otherwise specified, information on old-age pension for wage earners is retrieved from the National Pension office website at www.sfpd.fgov.be

${ }^{11}$ Second pillar pensions are voluntary, provided by the employer and established among the company, the industry or the sector of activity. Public authorities do not have direct control over second and third pillar pensions but define their legal framework and encourage their use through tax incentive mechanisms. ${ }^{12}$ See Royal Decree 50 of the $24^{\text {th }}$ of October 1967 - Arrêté Royal relatif à la pension de retraite et de survie des travailleurs salariés, M.B. 24 October 1967.

${ }^{13}$ Blue and white-collar workers were treated separately until 1962, when the legislator aligned the rules for every wage earner.

${ }^{14}$ Specific rules still exist for miners, marine workers, journalists and civil aviation personnel.

${ }^{15}$ See Law of the $29^{\text {th }}$ of December 1953 - Loi relative à la pension des ouvriers and the Royal Decree of the $24^{\text {th }}$ of October 1967 - Arrêté royal relatif à la pension de retraite et de survie des travailleurs salariés, M.B. 27 October 1967.

${ }_{16}$ Employee contributions amount to 13.07 percent of gross wage, employer contribution amount to 32.5 percent of 100 percent of the wage of white-collar workers and 108 percent of the wage of blue-collar workers.
} 
This section is structured as follows. Section 2.2.1 reviews the main components that enter the old-age pension benefits calculation and their evolution over time. Section 2.2.2 describes the evolution of the statutory and early eligibility ages and associated career conditions. Section 2.2.3 describes programs that adjust the final old-age pension benefit amount: the minimum pension, the actuarial adjustment and the pension bonus. Finally, section 2.2.4 is devoted to the description of the program that allows for the combination of work and old-age pension benefits receipt.

\subsubsection{CALCULATION OF BENEFITS}

The determination of the old-age pension benefits amount is based on three factors: the career length, the level of earnings and the household situation. A household can be classified into two categories: (i) households in which both members earn an income (hereafter two-earner households) and single individuals or (ii) households with only one prime earner (hereafter one-earner households) ${ }^{17}$. Each calendar year of employment increases the pension benefits entitlements by $1 / 45^{\text {th }}$ of 60 percent of the revalued gross wage in the case of two-earner or single households or 75 percent in the case of one-earner households.

The pension benefits calculation formula for an individual i can be represented by

$$
\text { Pension }_{i}=\frac{R R_{i}}{C L_{i}} \sum_{j}^{C} W_{i j} V C_{j}
$$

where index $i$ refers to the individual, index $\mathrm{j}$ refers to the year of career and $\mathrm{C}$ is the total career length. $W_{i j}$ refers to the nominal (in currency of the earning year) annual gross

\footnotetext{
${ }^{17}$ Households where both partners work but where the income of one of the spouse is below a certain threshold are also included in this group.
} 
wages received in year $\mathrm{j}$ by individual $\mathrm{i}$, subject to a set of floors and ceilings. $V C_{j}$ is the valorisation coefficient for year j. $C L_{i}$ is the standard career length for individual i. $R R_{i}$ is the replacement rate for individual i. We detail each of these components and their evolution over time in the sections below.

\subsubsection{WAGES}

Three types of wages are credited in the pension benefits calculation: the observed wage, the lump-sum wage and the assimilated wage.

The observed wage corresponds to the nominal gross wage the individual has earned for effective work, for which social security contributions were paid. Before 1968, only years of career of more than 185 days of at least 4 hours were credited in the old-age pension calculation. Starting from $1968^{18}$, this limitation is only applicable for years of career before 1955.

The lump-sum wage is fixed by year ${ }^{19}$ and replaces the observed wage for years worked as a blue-collar worker before $1955^{20}$ or as a white-collar worker before 1958 and for assimilated periods (periods during which a replacement income is received) before $1968 .{ }^{21}$

After $1967^{18}$, the assimilated wage ${ }^{22}$ is used to credit for assimilated periods in the pension benefits calculation: it is equivalent to the last observed wage received before the

\footnotetext{
18 See Royal Decree 50 of the $24^{\text {th }}$ of October 1967 - Arrêté Royal relatif à la pension de retraite et de survie des travailleurs salariés, M.B. 24 October 1967.

${ }^{19}$ See the appendix for the historical evolution of lump-sum wages.

${ }^{20}$ See the Law of the $21^{\text {st }}$ of May 1955 - Loi relative à la pension de retraite et de survie des ouvriers.

${ }^{21}$ The lump-sum wage also replaces observed wages in the case of seasonal or cross border work.

22 See appendix for the historical evolution of the assimilated wage.
} 
inactivity period. Starting from $2012^{23}$, the assimilated wage is limited to the legal minimum wage for periods of unemployment longer than two years ${ }^{24}$ (one year from 2017 onwards) and periods spent in the conventional early retirement program above age 58 (every age from 2017 onwards). Besides, since 2007, there exists a differentiated ceiling for the assimilated wage credited in the old-age pension benefit calculation for periods spent receiving unemployment, conventional early retirement or time credit benefits after age 58.

\subsubsection{VALORISATION COEFFICIENT}

The aim of the valorisation coefficient ${ }^{25}$ is to adjust past earnings to the price levels of the retirement year by adjusting them for inflation and average wage growth. ${ }^{26}$ It is equal to the pivotal inde $\mathrm{x}^{27}$ of the retirement year $\mathrm{R}$ divided by the average monthly price index of career year $\mathrm{j}$ and multiplied by the growth coefficient of career year $\mathrm{j}$. Below, we first discuss the pivotal index and then take a look at the growth coefficient.

$$
V C_{j}=\frac{\text { pivotal index }_{R}}{\text { average Price Index }} j
$$

The pivotal index is an automatic indexation mechanism of social security benefits to inflation: social security benefits increase by two percent if the price index reaches a

\footnotetext{
${ }^{23}$ See Law of the $28^{\text {th }}$ of December 2011 - Loi portant des dispositions diverses, M.B. 30 December 2011.

${ }^{24}$ This period usually corresponds to the start of the 3rd period of unemployment (between 12 and 36 months of unemployment) benefits during which benefits stop being linked to the lost wage and become a lump sum payment.

${ }^{25}$ See Law of the $28^{\text {th }}$ of March 1973 - Loi majorant les pensions des travailleurs salariés et instaurant un mécanisme d'adaptation du montant des pensions à l'évolution du bien-être général, M.B. 30 March 1973 and Law of the $1^{\text {st }}$ of March 1977 - Loi organisant un régime de liaison à l'indice des prix à la consommation du Royaume de certaines dépenses dans le secteur public, M.B. 12 March 1977.

${ }^{26}$ See the appendix for a list of valorisation coefficients for a person retiring in 2020 ( pivotal index 147.31).

27 See the appendix for the historical evolution of pivotal indices.
} 
threshold, the so-called pivotal index. Since 1994, the smoothed ${ }^{28}$ health index ${ }^{29}$ replaces the Consumer Price Index (CPI) as the price index used in the pivotal index determination. The health index is equivalent to the CPI from which the prices of tobacco, alcohol, diesel and fuel were removed. Therefore, once the smoothed health index reaches the pivotal index, social security benefits increase by two percent and remain unchanged until the smoothed health index reaches the newly defined pivotal index (the old pivotal index multiplied by 1.02). On four occasions, social security benefits did not increase after the pivotal index was reached: in 1983, 1984, 1985 and 2015. Following each of these jumps, the smoothed health index that enters the pivotal index determination is multiplied by a factor of 0.98 .

The use of the valorisation coefficient mechanism to revalue past earnings leads to an underestimation of past inflation. Indeed, the health index grows slower than the CPI because the prices of the products that are deducted from the CPI have historically been growing at a faster pace than other goods (see Figure 2.5). Moreover, the use of the smoothed index, the pivotal index mechanism and the four index jumps are also factors of this undervaluation. In fact, Peeters (2016) estimates that the valorisation coefficient used to adjust 1980 wages to 2016 prices is 12 percent lower than a fictitious indexation coefficient based on the CPI.

\footnotetext{
${ }^{28}$ Since 1983, the average of the last four months, called 'smoothed index' is used instead of the current index in order to avoid the impact of sudden fluctuations in prices on pension indexation.

${ }^{29}$ See Royal decree of the $24^{\text {th }}$ of December 1993 - Arrêté royal portant exécution de la loi du 6 janvier 1989 de sauvegarde de la compétitivité du pays, M.B. 31 March 1993. The detailed evolution of the health index is available on https://statbel.fgov.be/fr/themes/prix-la-consommation/indice-sante\#figures.
} 
Figure 2.5: Evolution of the CPI, the health index and the pivotal index (1996 - 2019)

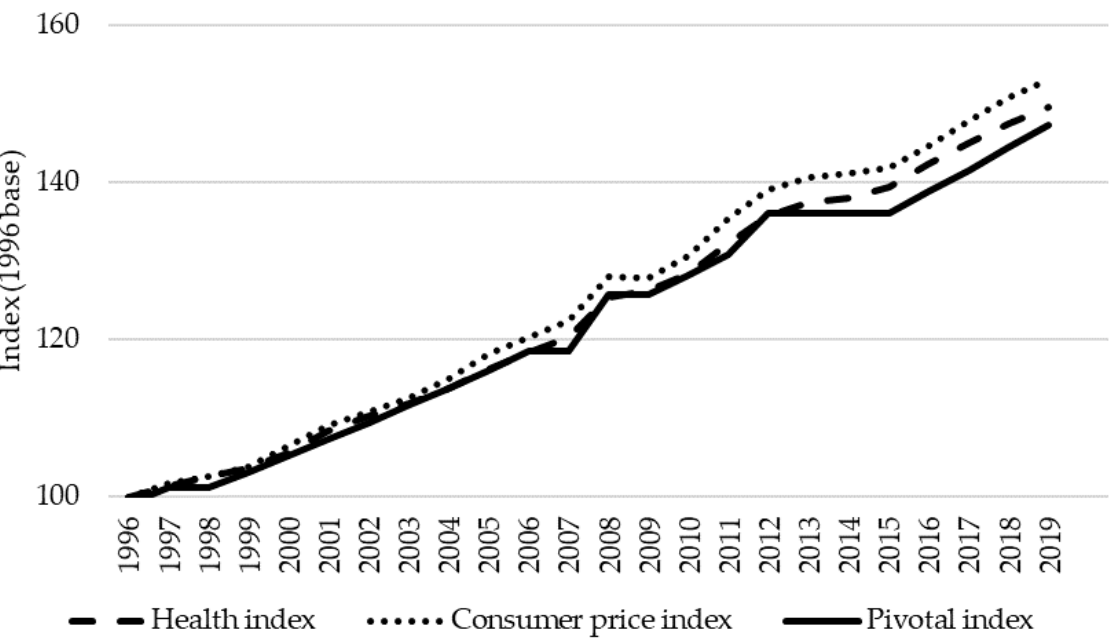

Source: Statbel ${ }^{30}$

The growth coefficient is the other important component of the valorisation coefficient. It is introduced in $1973^{25}$ and is aimed at indexing old-age pensions to wage growth. For retirement years after 1974, earnings between 1955 and 1974 were increased by 3.6 percent ${ }^{31}$ per year of difference between career year $j$ and $1975 .{ }^{32}$ Starting in 1997 , the base coefficient of 0.036 is gradually reduced by 0.004 per year. Consequently, the growth coefficient is equal to 1 from 2005 onwards (Feestjens, 1997).

\footnotetext{
${ }^{30}$ See 'Index search', https://statbel.fgov.be/en/themes/consumer-prices/index-search

${ }^{31}$ See Royal Decree 50 of the $24^{\text {th }}$ of October 1967 - Arrêté Royal relatif à la pension de retraite et de survie des travailleurs salariés, M.B. 24 October 1967.

32 See the appendix for the historical evolution of the growth coefficient.
} 
The growth coefficient for retirement year $\mathrm{j}$ or $\mathrm{k}$ and earnings year $\mathrm{i}$ is therefore determined as follows.

$$
\begin{gathered}
\text { Growth coefficient } t_{i j}=(1+0.036)^{1975-i} \\
\text { for } \mathrm{i}=1955, \ldots, 1974 \text { and } \mathrm{j}=1975, \ldots, 1996 \\
\text { Growth coefficient }_{i k}=(1+(0.036-(0.004 *(k-1996))))^{1975-i} \\
\text { for } \mathrm{i}=1955, \ldots, 1974 \text { and } \mathrm{k}=1997, \ldots, 2004
\end{gathered}
$$

Hence, unlike many European countries, earnings that enter the old-age pension benefits calculation are not automatically adjusted for wage growth ${ }^{33}$. Similarly, pensions in payment are not automatically adjusted for wage growth but only for (incomplete) inflation through the pivotal index. Consequently, the welfare gap between a retired individual and the average employed person increases with time spent in retirement. Nevertheless, discretional increases in pension benefits reflecting the evolution of living standards are periodically implemented. In fact, since 2005 a financial package aimed at increasing pensioners' well-being is formed every two years, with priority often granted to increases in minimum pensions. Additional payments called holiday and supplementary allowances are payable to pensioners once a year and are aimed at correcting for the lack of wage growth indexation.

\footnotetext{
33 See Dekkers, Desmet and Van den Bosch (2018) for a microsimulation of the impact of introducing a valorisation coefficient that accounts for increases in wage growth.
} 


\subsubsection{WAGE FLOORS}

A wage floor for earnings to be credited in the old-age pension benefit calculation is introduced in $1996^{34}$. Its objective is to avoid that gender wage inequality persists after retirement (Feestjens, 1997). The wage credited in the pension benefit calculation for a certain career year is replaced by the wage floor upon meeting three conditions: the wage is smaller than the wage floor, the worker has accumulated at least 104 days $^{35}$ of FTE (fulltime equivalent work) during that year and the total career length is at least 15 years ${ }^{36}$. Between 1978 and 1991, there was an additional condition: the ratio of the observed wage over the wage floor multiplied by 312 had to be superior to 104 . The wage floor is adjusted with the work intensity ratio ${ }^{37}$.

The wage floor to be applied at retirement is adjusted for inflation through the pivotal index mechanism. In addition, there have been various discretional increases in the wage floor amount to adjust it to wage growth. ${ }^{38}$ In particular, in $2009^{39}$, a substantial increase of the wage floor was decided to ensure that the old-age pension benefits amount of an individual with a career of 45 years and calculated on the basis of the wage floor was equal to the minimum pension. Table 2.1 and figure 2.6 display the evolution of the wage floor in real and nominal values.

\footnotetext{
${ }^{34}$ See Law of the $26^{\text {th }}$ of July 1996 - Loi portant modernisation de la sécurité sociale et assurant la viabilité des régimes légaux des pensions, M.B. 1 August 1996.

${ }^{35}$ One-third of full-time employment (312 days).

${ }^{36}$ Each year of career must be constituted of at least 104 days of FTE, periods spent receiving a replacement income included. See Royal Decree of the $23^{\text {rd }}$ of December 1996 - Arrêté royal portant exécution des articles 15 , 16 et 17 de la loi du 26 juillet 1996 portant modernisation de la sécurité sociale et assurant la viabilité des régimes légaux des pensions, M.B. 17 January 1997.

${ }^{37}$ The work intensity ratio is the total number of career years (of at least 52 days of FTE) divided by a complete career.

${ }^{38}$ At the time of writing, the wage floor is $25,833.78$ euros (pivotal index 147.31).

${ }^{39}$ See the Royal Decree of the $16^{\text {th }}$ of February 2009 - Arrêté royal portant augmentation du droit minimum par année de carrière et de certaines pensions dans le régime des travailleurs salariés, M.B. 13 March 2009.
} 
Table 2.1: Evolution of the wage floor per year of career (in constant 1996 euros)

\begin{tabular}{cc}
\hline Royal decree & Wage floor \\
\hline $23^{\text {rd }}$ of December 1996 & 509.916 BEF $(12,637.5 €)$ \\
$5^{\text {th }}$ November 2002 & $13,151.04 €$ \\
$16^{\text {th }}$ of February 2009 & $16,486.75 €$ \\
$6^{\text {th }}$ of July 2011 & $16,816.49 €$ \\
$24^{\text {th }}$ of June 2013 & $17,026.70 €$ \\
$3^{\text {rd }}$ of April 2015 & $17,367.23 €$ \\
$21^{\text {st }}$ of July 2017 & $17,662.47 €$ \\
$17^{\text {th }}$ of May 2019 & $18,088.35 €$ \\
\hline
\end{tabular}

Source: Moniteur Belge ${ }^{40}$ and the National Public Pension Office ${ }^{41}$

Figure 2.6: Evolution of wage floor in current euros

30000

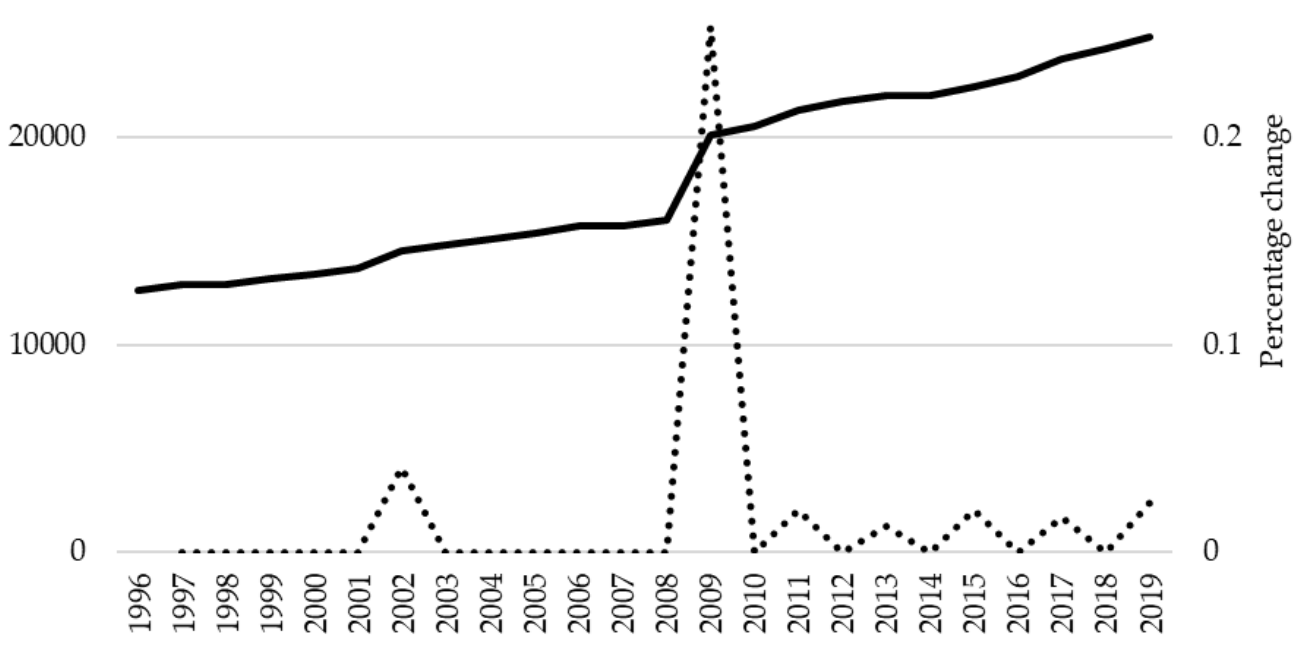

—Wage floor (nominal) ...... Percentage change

Source: Moniteur Belge ${ }^{40}$ and National Public Pension Office ${ }^{42}$

${ }^{40}$ See https://justice.belgium.be/fr/service_public_federal_justice/organisation/moniteur_belge ${ }^{41}$ See 'Droit minimum par année de carrière' on https://www.sfpd.fgov.be/fr/montant-de-lapension/calcul/types-de-pensions/salaries/salaires/droit-minimum

42 See https://www.sfpd.fgov.be/fr/centre-de-connaissances/legislation/legislation-pensions-salaries 


\subsubsection{CEILINGS}

Observed and assimilated wages that enter the old-age pension benefit calculation are subject to a set of ceilings. Above the wage ceiling, an additional euro earned will not lead to an increase in old-age pension benefits. Similar to the wage floors, the objective of ceilings is to avoid the persistence of gender wage inequality into old age. Initially, the ceilings for pensionable earnings were introduced with contribution ceilings for whitecollar workers and there was no ceiling for blue-collar workers. ${ }^{43}$ In 1982, contribution ceilings and ceilings for wages in the individual pension accounts were abolished. In 1983, wage ceilings were introduced for blue-collar workers for career years after $1981 .{ }^{4}$

At retirement, the wage in nominal value is compared to the ceiling for pensionable earnings of that year (adjusted for work intensity). If the wage is above the ceiling, the wage is replaced by the ceiling, adjusted for work intensity, which is subsequently revalued in the old-age pension calculation using the valorisation coefficient. Between 1958 and 1972, if the pensionable wage went over the ceiling, it was replaced by the ceiling amount adjusted for work intensity and multiplied by 1.1.

The ceilings are tied to a specific year and are automatically indexed to prices using the health index. The indexation of ceilings is therefore slightly less underestimated compared to that of the wage floor, which is adjusted using the pivotal index. Nevertheless, the use of the health index instead of the CPI still leads to an incomplete indexation to prices. On top of this indexation, there were several discretional increases of ceiling amounts in order to reflect the average wage growth. ${ }^{45}$ However, Peeters (2016)

\footnotetext{
${ }^{43}$ See the appendix for the historical evolution of the ceilings for pensionable earnings.

${ }^{44}$ Marine workers and flying staff still have a different set of pensionable earnings ceilings.

${ }^{45}$ See Law of the $26^{\text {th }}$ of July 1996 - Loi portant modernisation de la sécurité sociale et assurant la viabilité des régimes légaux des pensions, M.B. 1 August 1996.
} 
observes that ceilings have grown at a slower pace compared to the average wage for most age groups. Consequently, the proportion of individuals with earnings above the ceiling has been increasing over the years. ${ }^{46}$ Figure 2.7 presents the evolution of the wage ceiling in constant 1996 euros.

Figure 2.7: Evolution of pensionable earnings ceilings

$$
70,000
$$

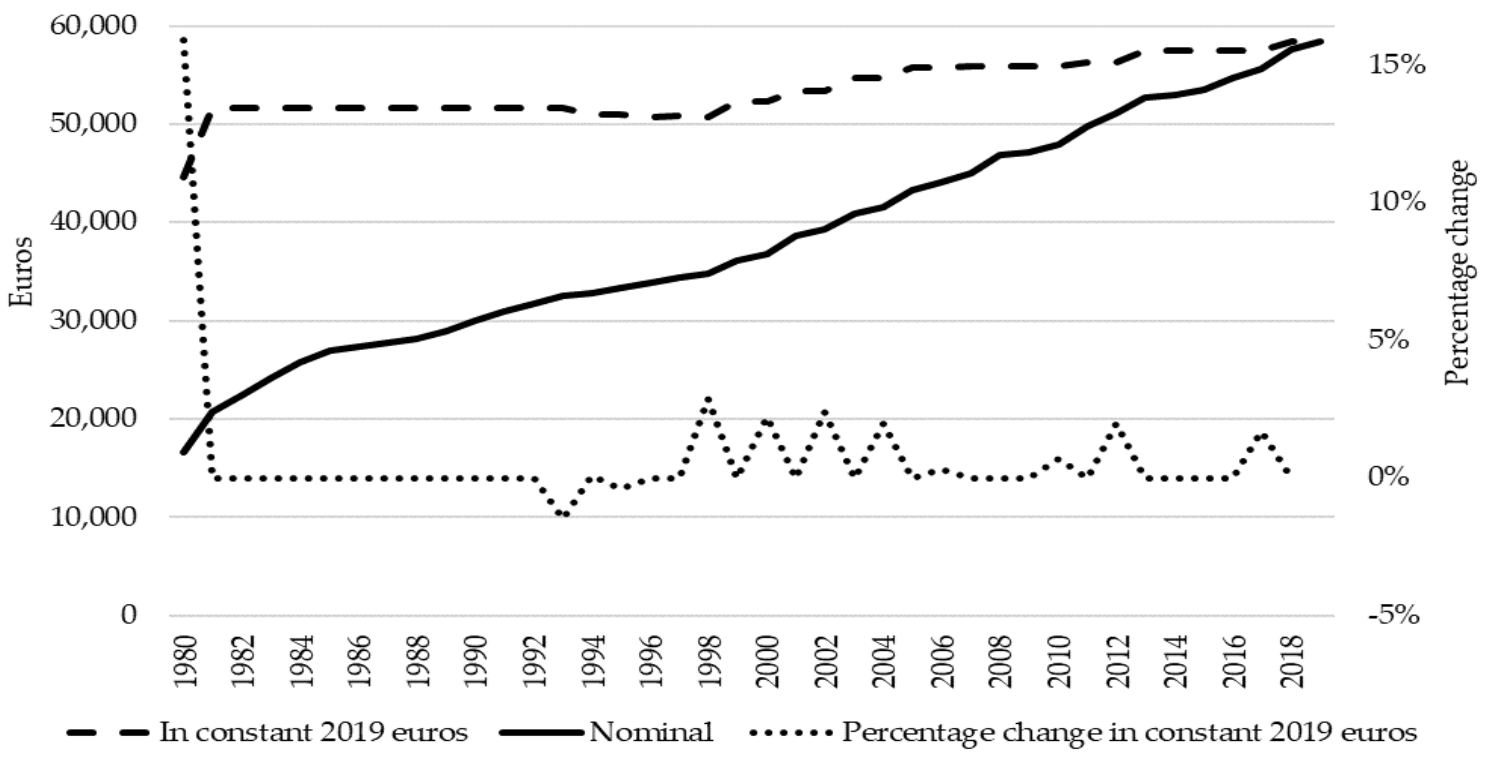

Source: National Public Pension Office ${ }^{42}$

The combination of pensionable earning ceilings and the inclusion of a maximum of 14,040 days of FTE lead to an implicit maximum pension amount (see section 2.2.1.6). The maximum pension for a pensioner who has a career of 14,040 days and who retires in 2019 was 2,628.38 euros for an individual in a two-earner or single household and 3,285.48 euros for an individual in a one-earner household. ${ }^{47}$ Peeters (2016) shows that the

\footnotetext{
${ }^{46}$ In 2008, it is estimated that 20.1 percent of pensioners saw their pensionable earnings limited because of the ceilings. On average, 16 years of pensionable earnings are limited for men and 11.2 years for women, this corresponds to an average pension benefits loss of 26.27 percent for men and 11.24 percent for women. (Pension Reform Committee 2020-2040, 2014)

${ }_{47}$ Blue-collar workers can earn a pension that is above the maximum pension because the pensionable earnings ceiling only applies to wages earned after 1981.
} 
maximum pension has nearly doubled since 1980 following discretional increases in ceilings and the gradual replacement of relatively meager lump-sum rate wage that were included in the pension benefits calculation before 1955 for white-collar workers and before 1958 for blue collar workers (see section 2.1.1) instead of the observed wage.

\subsubsection{REPLACEMENT RATE}

Initially, the standard replacement rate for old-age pension benefits, hereafter referred to as the isolated replacement rate, was set at 40 percent. The household replacement rate of 60 percent is introduced for married workers with a financially dependent spouse in $1953^{48}$ for blue-collar workers and in $1957^{49}$ for white-collar workers. In $1955^{50}$, the isolated replacement rate is raised to 60 percent and the household replacement rate is raised to 75 percent. ${ }^{51}$

To receive an old-age pension calculated with the household replacement rate, the financially dependent spouse's work income cannot go over a certain earnings threshold ${ }^{52}$ and he or she also cannot receive any type of social security benefits, except for an oldage pension. In the latter case, the sum of both spouses' old-age pensions must be lower than the pension of the prime earner calculated at the household replacement rate. If that is the case, the pension benefits of the prime earner are automatically topped up to the

\footnotetext{
${ }^{48}$ See the law of the $29^{\text {th }}$ of December 1953 - Loi relative à la pension des ouvriers.

${ }^{49}$ See the law of the $12^{\text {th }}$ of July 1957 - Loi relative à la pension de retraite et de survie des employés.

${ }^{50}$ See the law of the $21^{\text {st }}$ of May 1955 - Loi relative à la pension de retraite et de survie des ouvriers.

${ }^{51}$ Because past earnings are not fully adjusted for inflation and wage growth, Berghman and Peeters (2012) estimate the real replacement rate to be 42 percent for an individual receiving the average wage, 60.1 percent for an individual receiving 50 percent of the average wage because of the wage floor and the minimum pension and 32.7 percent for individuals earning 150 percent of the average wage because of pensionable earnings ceilings.

52 The earnings threshold is similar to that for the combination of work and pension receipt. See Royal Decree of the 20st of January 2015 - Arrêté Royal modifiant l'article 64 de l'arrêté royal du 21 décembre 1967 portant règlement general du regime de pension de retraite et de survie des travailleurs salaries, M.B. 23 January 2015.
} 
pension amount calculated at the household replacement rate - producing an effective benefit replacement rate between 60 and 75 percent. If the sum of both spouse's old-age pension go over the pension of the prime earner calculated at the household replacement rate, the pension of the prime earner is paid at the isolated replacement rate.

\subsubsection{COMPLETE CAREER}

The condition for receiving a full pension is a complete career. A complete career is defined as a career of 45 years (of at least 104 days of FTE each) for men and 40 years for women since $1953^{53}$ for blue-collar workers and since $1957^{54}$ for white-collar workers. In $1996^{55}$, following a continuous decrease in the gender gap in total career years, complete career years for women are increased with the objective of harmonizing the system between men and women (Peeters, 2016). The transition period lasted from 1997 to 2009, during which complete career years for women increased from 40 to 41 in 1997, to 42 in 2000, to 43 in 2003, to 44 in 2006 and to 45 in 2009.

Before 2015, only the 45 years that lead to the highest pension accrual were retained in the pension benefits calculation (replacement income included). ${ }^{56}$ Since $2015^{57}$, this 45 years threshold is translated into $14,040^{58}$ days. The objective of this reform is to avoid that an

\footnotetext{
${ }^{53}$ See Law of the $29^{\text {th }}$ of December 1953 - Loi relative à la pension des ouvriers.

${ }^{54}$ See Law of the $12^{\text {th }}$ of July 1957 - Loi relative à la pension de retraite et de survie des employés.

55 See Law of the $26^{\text {th }}$ of July 1996 - Loi portant modernisation de la sécurité sociale et assurant la viabilité des régimes légaux des pensions, M.B. 1 August 1996.

${ }^{56}$ In 2008, the old-age pension of 41.9 percent of men and 18.7 percent of women were reduced because of a career longer than the complete career length. On average, 3.6 years for men and 2.9 years for women were removed from their total career years (Pension Reform Committee 2020-2040, 2014).

${ }^{57}$ See Law of the $24^{\text {th }}$ April 2014. - Loi modifiant diverses dispositions relatives au régime de pension des travailleurs indépendants compte tenu du principe de l'unité de carrière, M.B. 7 May 2014 and the Royal Decree of the $6^{\text {th }}$ of October 2015 - - Arrêté royal modifiant l'article 52 de l'arrêté royal du 21 décembre 1967 portant règlement général du régime de pension de retraite et de survie des travailleurs salariés, MB. 21 November 2015.

${ }_{58} 14,040$ is the result of 45 years multiplied by 312 days. Although a five-days working week is mandatory since 1964, working days are still translated into a six-days working week by multiplying the effective number of working days by 1.2. This leads to a full-time employment year being counted as 312 days (52
} 
individual with a career of 45 years of part-time work has the same work intensity ratio than another individual with a career of 45 years of full-time work. Therefore, since 2015, only the 14,040 days of work with the highest pension accrual are kept in the pension benefits calculation. Above 14,040 days, the assimilated wage of the conventional early retirement or the unemployment benefits cannot be included into the pension benefit calculation, even if they are more beneficial ${ }^{59}$.

\subsubsection{STATUTORY AND EARLY ELIGIBILITY AGES}

The statutory eligibility age is set at 65 for men and 60 for women in $1953^{53}$ for blue-collar workers and in $1957^{54}$ for white-collar workers. In 199760, a major pension reform progressively increased the statutory eligibility age of women to 65 with the aim of harmonizing the system between men and women and of improving the sustainability of the pension system. The statutory eligibility age for women increased from 60 to 61 in 1997, 62 in 2000, 63 in 2003, 64 in 2006 and 65 in 2009. The statutory eligibility age of both men and women, currently fixed at 65, will increase to 66 in 2025 and to 67 in $2030 .{ }^{61}$

The regulation regarding early retirement has been profoundly modified over the years. In $1967^{62}$, blue-collar workers could claim their old-age pension benefits 5 years before the statutory eligibility age, but they were subject to a 5 percent reduction of their pension

multiplied by 6) instead of the actual 260 working days (52 multiplied by 5). Therefore, with this administrative modification, a full career of 45 years is translated into 14,040 days and not 11,700 days (Peeters, 2016).

${ }^{59}$ Nevertheless, no more than 1,560 days of assimilated wage can be suppressed.

${ }^{60}$ See Royal Decree of the 23 ${ }^{\text {rd }}$ of December 1996 - Arrêté royal portant exécution des articles 15, 16 et 17 de la loi du 26 juillet 1996 portant modernisation de la sécurité sociale et assurant la viabilité des régimes légaux des pensions, M.B. 17 January 1997.

${ }^{61}$ See Law of the $10^{\text {th }}$ of August 2015 - Loi visant à relever l'âge légal de la pension de retraite et portant modification des conditions d'accès à la pension de retraite anticipée et de l'âge minimum de la pension de survie, M.B. 21st of August 2015.

${ }^{62}$ See Royal Decree of the 24th of October 1967 - Arrêté Royal relatif à la pension de retraite et de survie des travailleurs salariés, M.B. 24 October 1967. 
benefits per year of anticipation (see section 2.2.3.2). In 198163, a career requirement of 10 years (of minimum 104 days of FTE each) as a wage earner is introduced to access early retirement. In $1987^{64}$, the early eligibility age of women is increased to 60 . In 199165, a flexible retirement age starting at 60 without penalty is created. From 1997 to 2005, the career requirement for early retirement is gradually increased from 20 to 35 years by gradual steps of two years. From 2013 to $2016^{66}$, the early eligibility age is increased by 0.5 every year to reach 62 in 2016 . From 2013 to $2015^{66}$, the career conditions are increased by one every year to reach 40 years in 2015. Some exceptions are created for individuals with long career: age 60 with at least 40 years of career in 2013 and 2014, age 60 with at least 41 years of career in 2015, age 60 with at least 42 years of career or age 61 with at least 41 years of career in 2016. Starting in $2015^{61}$, the early eligibility age is increased by 0.5 every year to reach 63 in 2019. The career conditions for early retirement increase to 41 years in 2017 and to 42 years in 2019. Some exceptions are again created for individuals who have long careers: age 60 with a career of at least 43 years in 2017 and 2018, age 61 with a career of at least 43 years in 2017 and 2018, age 60 with a career of at least 44 years in 2019 and age 61 with a career of at least 43 years in 2019.

\footnotetext{
${ }^{63}$ See Law of the $10^{\text {th }}$ of February 1981 - La loi de redressement relative aux pensions $d u$ secteur social $d u$ 10.02.1981, M.B. 14 February 1981.

${ }^{64}$ See Royal Decree of the $16^{\text {th }}$ of July 1986 - Arrêté royal modifiant certaines dispositions en matière de pensions pour travailleurs salariés, M.B. 30 July 1986.

${ }^{65}$ See Law of the $20^{\text {st }}$ of July 1990 - Loi instaurant un âge flexible de la retraite pour les travailleurs salariés et adaptant les pensions des travailleurs salariés à l'évolution du bien-être général, M.B. 15 August 1990.

${ }_{66}$ See Law of the 28 $8^{\text {th }}$ of December 2011 - Loi portant des dispositions diverses, M.B. 30 December 2011.
} 
Table 2.2: Evolution of early and statutory eligibility ages and career length requirements

\begin{tabular}{|c|c|c|c|c|}
\hline & \multicolumn{2}{|c|}{$\begin{array}{c}\text { Early eligibility age } \\
\text { (Career requirements) }\end{array}$} & \multicolumn{2}{|c|}{ Statutory eligibility age } \\
\hline & Standard rule & Long career & Men & Women \\
\hline Before 1997 & $\mathrm{~N} / \mathrm{A}$ & N/A & 65 & 60 \\
\hline 1997 & $60(20)$ & $\mathrm{N} / \mathrm{A}$ & 65 & 61 \\
\hline 1998 & $60(22)$ & $\mathrm{N} / \mathrm{A}$ & 65 & 61 \\
\hline 1999 & $60(24)$ & $\mathrm{N} / \mathrm{A}$ & 65 & 61 \\
\hline 2000 & $60(26)$ & $\mathrm{N} / \mathrm{A}$ & 65 & 62 \\
\hline 2001 & $60(28)$ & $\mathrm{N} / \mathrm{A}$ & 65 & 62 \\
\hline 2002 & $60(30)$ & $\mathrm{N} / \mathrm{A}$ & 65 & 62 \\
\hline 2003 & $60(32)$ & $\mathrm{N} / \mathrm{A}$ & 65 & 63 \\
\hline 2004 & $60(34)$ & $\mathrm{N} / \mathrm{A}$ & 65 & 63 \\
\hline 2005 & $60(35)$ & $\mathrm{N} / \mathrm{A}$ & 65 & 63 \\
\hline 2006 & $60(35)$ & $\mathrm{N} / \mathrm{A}$ & 65 & 64 \\
\hline 2007 & $60(35)$ & $\mathrm{N} / \mathrm{A}$ & 65 & 64 \\
\hline 2008 & $60(35)$ & $\mathrm{N} / \mathrm{A}$ & 65 & 64 \\
\hline 2009 & $60(35)$ & $\mathrm{N} / \mathrm{A}$ & 65 & 65 \\
\hline 2010 & $60(35)$ & $\mathrm{N} / \mathrm{A}$ & 65 & 65 \\
\hline 2011 & $60(35)$ & $\mathrm{N} / \mathrm{A}$ & 65 & 65 \\
\hline 2012 & $60(35)$ & N/A & 65 & 65 \\
\hline 2013 & 60 and $1 / 2(38)$ & $60(40)$ & 65 & 65 \\
\hline 2014 & $61(39)$ & $60(40)$ & 65 & 65 \\
\hline 2015 & 61 and $1 / 2(40)$ & $60(41)$ & 65 & 65 \\
\hline 2016 & $62(40)$ & $\begin{array}{c}60(42) \text { or } 61 \\
(41)\end{array}$ & 65 & 65 \\
\hline 2017 & 62 and $1 / 2(41)$ & $\begin{array}{c}60(43) \text { or } 61 \\
(42)\end{array}$ & 65 & 65 \\
\hline 2018 & $63(41)$ & $\begin{array}{c}60(43) \text { or } \\
61(42)\end{array}$ & 65 & 65 \\
\hline 2019 & $63(42)$ & $\begin{array}{c}60(44) \text { or } \\
61(43)\end{array}$ & 65 & 65 \\
\hline
\end{tabular}

Source: National Public Pension Office (2017), Feestjens (1997), OECD (2017) 


\subsubsection{ADJUSTMENTS OF PENSION AMOUNTS}

\subsubsection{MINIMUM PENSION}

The minimum pension is introduced in $1980^{67}$ for workers with a career of at least 45 years. Differentiated minimum pension amounts exist for one-earner households and for survivor pensions. In $1981^{68}$, the career length requirement for the minimum pension decreases to 30 years of career of at least 208 days $^{69}$ of FTE each. Minimum pension amounts are adjusted with the work intensity ratio ${ }^{70}$ and for inflation through the pivotal index mechanism..$^{71}$

Introduced in $2006^{72}$, the part-time minimum pension system offers more flexible eligibility criteria: at least 30 years of at least 156 days of FTE each. ${ }^{73}$ If such conditions are met, then the individual is entitled to receive the part-time minimum pension amount multiplied by the work intensity ratio $^{74}$ expressed in days. Table 2.3 summarizes the

\footnotetext{
${ }^{67}$ See Law of the $8^{\text {th }}$ of August 1980 - Loi relative aux propositions budgétaires 1979-1980, M.B. 15 August 1980. There also exist a means tested social pension based on residency in Belgium (called the GRAPA) introduced in 1970.

${ }^{68}$ See the Law of the $10^{\text {th }}$ of February 1981 - La loi de redressement relative aux pensions $d u$ secteur social $d u$ 10.02.1981, M.B. 14 February 1981.

${ }^{69} 208$ is equal to two-thirds of a full time employment equivalent (312 days).

70 The work intensity ratio is the number of career years (of at least 52 days of FTE) divided by a complete career (45 years in most cases).

${ }^{71}$ In 2020 (pivotal index 147.31), the minimum pension (for a complete career) is 15,500.27 euros at the isolated replacement rate, 19,369.22 euros at the household replacement rate and 15,293.11 euros for the survivor pension.

${ }^{72}$ See Royal decree of the $28^{\text {th }}$ of September 2006 - Arrêté royal portant exécution des articles 33, 33bis, 34 et $34 b i s$ de la loi de redressement du 10.02.1981 relative aux pensions du secteur social, M.B. 6 October 2006.

${ }^{73}$ Special eligibility conditions for mixed careers allow the inclusion of years worked as self-employed in the required 30 years of career.

${ }^{74}$ The work intensity ratio in days is the sum of days worked (only for career years of at least 52 days of FTE) over a complete career expressed in days (14,040 days). Assimilated days can be included in the numerator.
} 
discretional increases in real values (index base 1988 or 1996) of the minimum pension amount for the old-age pension.

Table 2.3: List of royal decrees modifying the minimum pension amount

\begin{tabular}{ccc}
\hline \multirow{2}{*}{ Royal decree } & \multicolumn{2}{c}{ Old-age pension } \\
\cline { 2 - 3 } & $\begin{array}{c}\text { One-earner } \\
\text { household }\end{array}$ & $\begin{array}{c}\text { Two-earner } \\
\text { household }\end{array}$ \\
\hline $15^{\text {th }}$ of May 1984 & 214644 BEF & $171765 \mathrm{BEF}$ \\
& $(5,319.63 €)$ & $(4,256.94 €)$ \\
$14^{\text {th }}$ of May 2000 & $242643 \mathrm{BEF}$ & $194179 \mathrm{BEF}$ \\
& $(6,013.54 €)$ & $(4,812.43 €)$ \\
$11^{\text {th }}$ of December 2001 & $11,113.56 €$ & $8,893.80 €$ \\
$14^{\text {th }}$ of February 2003 & $11,535.12 €$ & $9,231 €$ \\
$9^{\text {th }}$ of April 2007 & $11,765.82 €$ & $9,415.62 €$ \\
$12^{\text {th }}$ of June 2008 & $12,001.14 €$ & $9,603.93 €$ \\
$16^{\text {th }}$ of February 2009 & $12,361.17 €$ & $9,892.05 €$ \\
$6^{\text {th }}$ of April 2011 & $12,608.39$ & $10,089.89$ \\
$24^{\text {th }}$ of June 2013 & $12,765.99 €$ & $10,216.01 €$ \\
$3^{\text {rd }}$ of April 2015 & $13,021.31 €$ & $10,420.33 €$ \\
$2^{\text {st }}$ of July 2017 & $13,151.52 €$ & $10,524.53 €$ \\
$17^{\text {th }}$ of May 2019 & $13,283.04 €$ & $10,629.78 €$ \\
\hline
\end{tabular}

Source: Moniteur belge ${ }^{40}$ and National Public Pension Office ${ }^{42}$ 


\subsubsection{PENSION PENALTY}

In $1967^{75}$, a reduction of 5 percent of old-age pension benefits per year of anticipation before the statutory eligibility age is created. In $1976^{76}$, an exception is created for individuals who retire at age 64 and can prove a career of 45 years as wage-earner. Additionally, and exception was also created for individuals who worked in harsh conditions, they could retire at age 64 without penalty. In $1982^{77}$, the 5 percent reduction is removed if the worker is laid off and the employer replaces the retiring worker with an unemployed worker. Between $1978^{78}$ and 1982, individuals who receive unemployment or disability benefits can claim old-age pension benefits without penalty from age 60 onwards for men and age 55 onwards for women. In 198779, no anticipation is allowed below age 60 . In $1991^{80}$, the pension penalty is eliminated. Hence, no penalty is currently applied for early retirement.

\footnotetext{
${ }^{75}$ See Royal decree of the $24^{\text {th }}$ of October 1967-Arrêté royal relatif à la pension de retraite et de survie des travailleurs salariés, M.B. 27 October 1967.

${ }^{76}$ See Law of the $27^{\text {th }}$ of February 1976 - Loi modifiant l'arrêté royal $n^{\circ} 50$ du 24.10 .1967 relatif à la pension de retraite et de survie des travailleurs salariés., M.B. 9 March 1976.

${ }_{77}$ See Royal decree of the $28^{\text {th }}$ of September 1982 - Arrêté Royal relatif à la prépension de retraite des travailleurs salariés, M.B. 29 September 1982.

${ }^{78}$ See Law of the 22 ${ }^{\text {nd }}$ of December 1977 - Loi relative aux propositions budgétaires 1977-1978, M.B. 24 December 1977.

${ }^{79}$ See the Royal Decree of the $16^{\text {th }}$ of July 1986 - Arrêté royal $n^{\circ} 415$ modifiant certaines dispositions en matière de pensions pour travailleurs salariés, M.B. 30 July 1986.

80 See the Law of the $20^{\text {st }}$ of July 1990 - Loi instaurant un âge flexible de la retraite pour les travailleurs salariés et adaptant les pensions des travailleurs salariés à l'évolution du bien-être général, M.B. 15 August 1990.
} 


\subsubsection{PENSION BONUS}

The pension bonus is introduced in $2007^{81}$ with the objective of encouraging older workers to stay on the labor force longer. ${ }^{82}$ In order to benefit from the pension bonus, the worker must continue working above age 62 or after 44 years of career. The pension bonus is also granted to beneficiaries of survivor pensions if the deceased spouse fulfils the eligibility conditions before his or her passing. The bonus is initially set at $2 \operatorname{euros}^{83}$ per day of effective full-time work (outside of assimilated periods). It is adjusted to inflation through the pivotal index but it is not adjusted for average wage growth.

Thanks to its lump-sum nature, the pension bonus serves as a redistribution mechanism. Indeed, the financial impact of the pension bonus on old-age pensions is relatively higher for low earners then for high earners. ${ }^{84}$ Figure 2.8 displays the percentage increase in oldage pension benefits with pension bonus benefits (2007 rules) for different initial pension amounts. Unsurprisingly, the impact of the pension bonus is largest for the lower initial pension amount and the effect of longer eligibility periods is heightened for lower oldage pension amounts.

\footnotetext{
${ }^{81}$ See the Law of the $23^{\text {rd }}$ of December 2005 - Loi relative au pacte de solidarité entre les générations, M.B. 30 December 2005 and the Royal Decree of the $1^{\text {st }}$ of February 2007 - Arrêté royal instituant un bonus de pension, M.B. 9 February 2007.

82 In 2011, there were 47,894 men and 19,794 women who received pension bonus, or 20 percent and 10 percent of total men and women pensioners aged between 62 and 68, respectively (Conseil Supérieur des Finances, 2012). The gross monthly average amount of the pension bonus was 116 euros for men and 91 for women. It was proven that the impact of the pension bonus on the labor force participation of the elderly was low in comparison to reforms that increased the career requirements for early retirement (Pension Reform Committee 2020-2040, 2014).

${ }^{83}$ In 2020, the pension bonus per day is set at 2.4868 euros (index 147.31).

${ }^{84}$ As an example, a pension of 1000 euros in 2007 would increase by 5.2 percent with 12 months of pension bonus and by 15.6 percent with 36 months of pension bonus. The maximum pension in 2007 (1769.38 monthly euros) would increase by 3.5 percent with 12 months of pension bonus and 8.8 percent with 36 months of pension bonus.
} 
Figure 2.8: Impact of the pension bonus on old-age pension benefits- 2007 rules

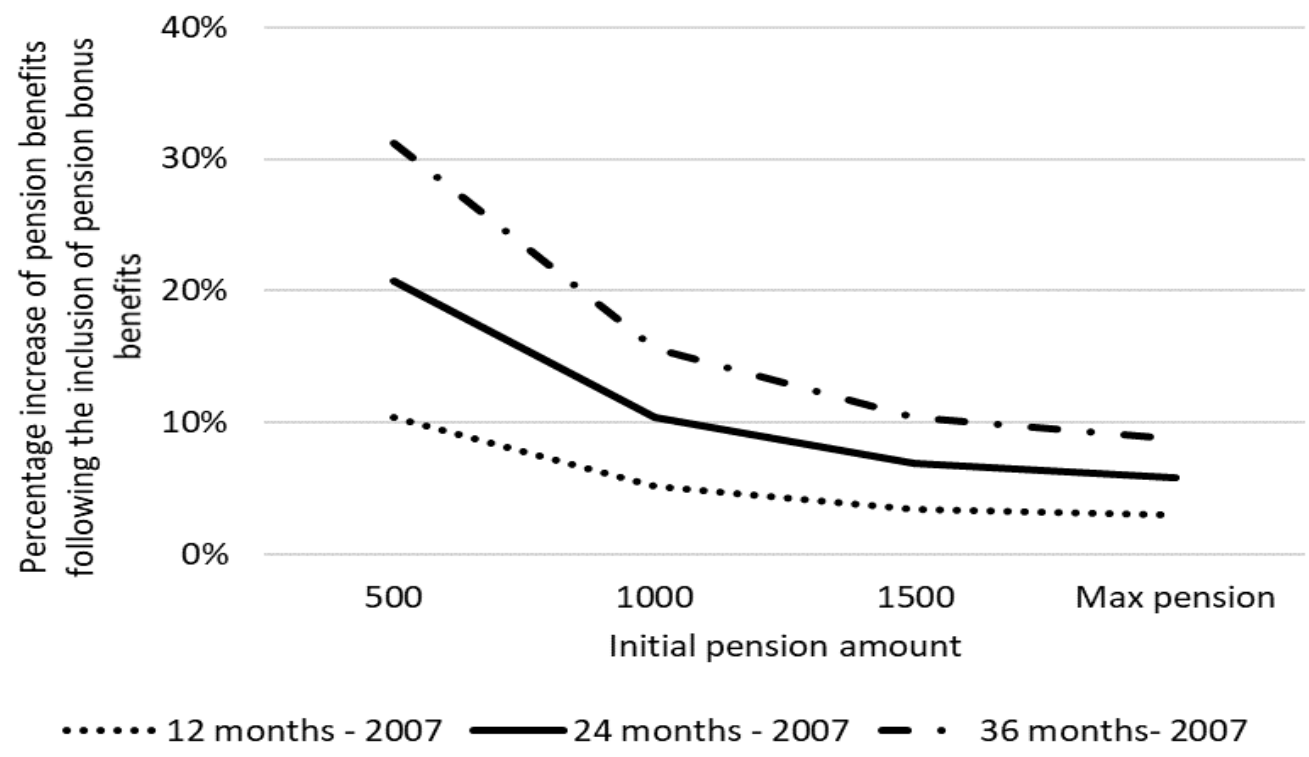

Source: Author's own calculations and National Public Pension Office ${ }^{85}$

Initially, the pension bonus was set up to be a temporary measure, which had to be evaluated by the Pension Reform Committee before the $1^{\text {st }}$ of December 2012. In view of its limited effect on employment rates ${ }^{86}$ and to reduce the financial burden it caused, pension bonus rules were modified for new pensions in 2014. Starting in 2014 ${ }^{87}$, the pension bonus amount increases with the length of the reference period (see table 4). The reference period starts on the first day of the $12^{\text {th }}$ month after the wage-earner becomes eligible for early retirement or after the worker has reached the age of 65 with at least 40 years of career. Besides, under the new regulations, only days of effective work are considered; thus, no assimilated periods count for the eligibility to the pension bonus. Finally, the pension bonus becomes a personal pension entitlement and can no longer be

\footnotetext{
${ }^{85}$ See https://www.sfpd.fgov.be/fr/montant-de-la-pension/calcul/bonus-de-pension\#salarie ${ }^{86}$ See (Pension Reform Committee 2020-2040, 2014) for the analysis of the impact of the pension bonus on the retirement behavior of older Belgian workers.

${ }^{87}$ See the Royal Decree of the $24^{\text {th }}$ of October 2013 - Arrêté Royal portant exécution, en matière de bonus de pension des travailleurs salariés, de l'article 7 bis de la loi du 23 décembre 2005 relative au pacte de solidarité entre les générations., MB 6 November 2013.
} 
transferred to another person in the case of the survivor pension. Since $2015^{88}$, the pension bonus is abolished for new pensioners. However, pensioners who can prove that they fulfilled the 2014 eligibility criteria for pension bonus before December 1, 2014 can still receive pension bonus benefits. Figure 2.9 displays the percentage increase in old-age pension benefits (2014 rules) with pension bonus benefits for different initial pension amounts. As in figure 2.8, the effect of the pension bonus decreases as the pension amount increases. Moreover, we observe that its impact is lower on old-age pension compared to 2007 rules.

Table 2.4: Pension bonus benefits amounts - 2014 rules

\begin{tabular}{cc}
\hline $\begin{array}{c}\text { Amount of pension bonus } \\
\text { per day of effective work } \\
\text { (pivotal index 136.09, base }\end{array}$ & Reference period \\
1996 ) & \\
$€ 1.5$ & First 12 months \\
$€ 1.7$ & From $13^{\text {th }}$ to $24^{\text {th }}$ month \\
$€ 1.9$ & From $25^{\text {th }}$ to $36^{\text {th }}$ month \\
$€ 2.1$ & From $37^{\text {th }}$ to $48^{\text {th }}$ month \\
$€ 2.3$ & From $49^{\text {th }}$ to $60^{\text {th }}$ month \\
$€ 2.5$ & From $61^{\text {st }}$ month onwards \\
\hline \hline
\end{tabular}

Source: Moniteur Belge ${ }^{40}$ and the Royal Decree of the 24th of October 2013 - Arrêté royal portant exécution, en matière de bonus de pension des travailleurs salariés, de l'article $7 b i s$ de la loi du 23 décembre 2005 relative au pacte de solidarité entre les générations, M.B. 6 November 2013.

${ }^{88}$ See the Law of the 19th of December 2014 - Loi-programme, M.B. 29 December 2014. 
Figure 2.9: Impact of the pension bonus on old-age pension amounts- 2014 rules

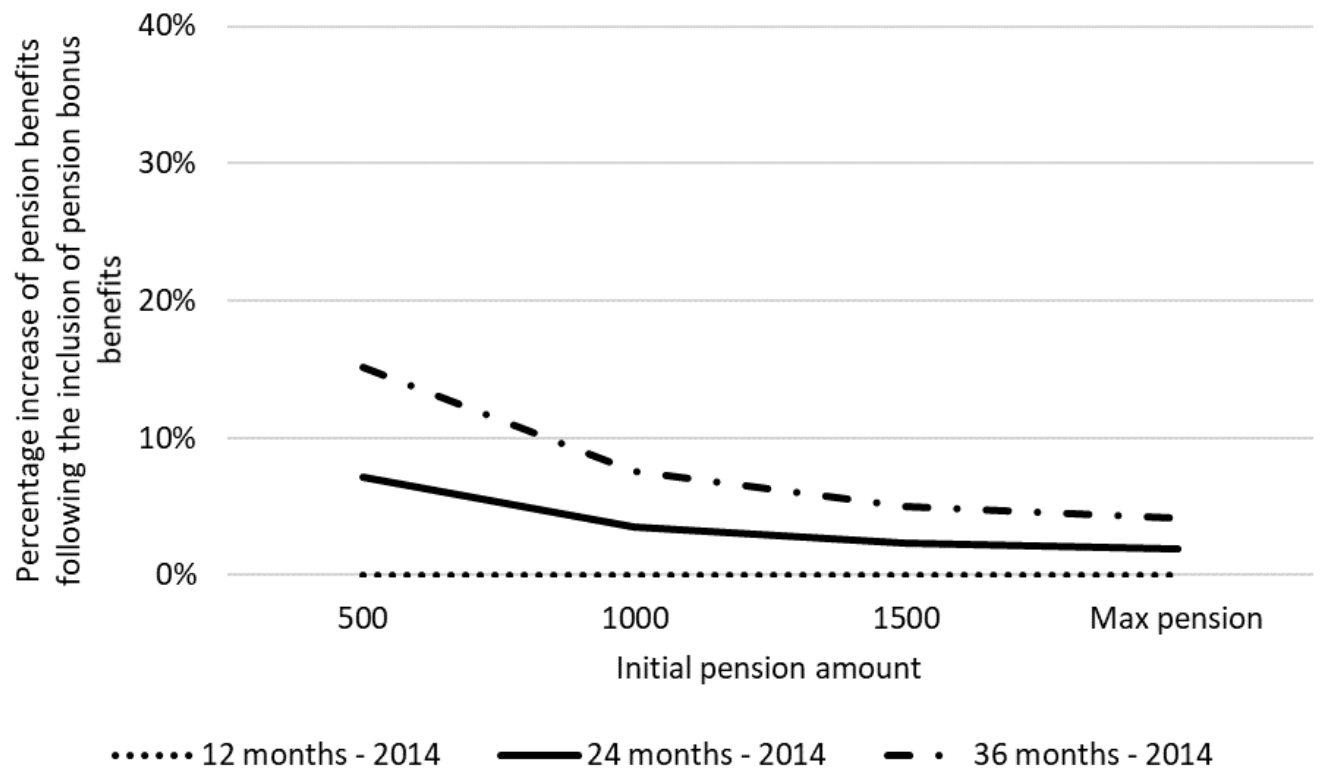

Source: Author's own calculations and the National Public Pension Office ${ }^{85}$

\subsubsection{COMBINATION OF WORK AND OLD-AGE PENSION BENEFITS}

Receiving old-age pension benefits and continuing working is possible. However, partial old-age pension receipt while remaining on the labor market is not. Thus, the additional wage earned will not lead to an increase in pension benefits.

The earnings threshold for work after retirement is introduced in $1967^{89}$. From $2013^{90}$ onwards, the earnings threshold is eliminated for workers above the age of 64 with at

\footnotetext{
${ }^{89}$ See the Royal decree of the $24^{\text {th }}$ of October $1967-$ Arrêté royal relatif à la pension de retraite et de survie des travailleurs salariés, M.B. 27 October 1967.

${ }^{90}$ See the Royal Decree of the $28^{\text {th }}$ of May 2013 - Arrêté royal modifiant diverses dispositions réglementaires relatives au cumul d'une pension dans le régime des travailleurs salariés avec des revenus professionnels ou des prestations sociales, M.B. 20 June 2013.
} 
least 42 years of career ${ }^{91}$. From $2015^{92}$ onwards, the earnings threshold is removed for individuals above the age of 64 or for individuals with more than 44 years of career ${ }^{91}$. If the earnings exceed the threshold by less than 100 percent of the threshold, then pension benefits are reduced by similar percentage points. If the earnings exceed the threshold by more than 100 percent of the threshold, then the pension is suspended. ${ }^{93}$

\subsection{CONVENTIONAL EARLY RETIREMENT ${ }^{94}$}

The conventional early retirement program ${ }^{95}$ is introduced in $1974^{96}$ with the aim of facilitating the restructuration of the industrial sector and the replacement of older workers with younger and unemployed workers. In $1975^{97}$, conventional early retirees are granted a special unemployed status upon which they are entitled to receive unemployment benefits. Next to the standard conventional early retirement regime, numerous sector-specific or job-specific conventional early retirement regimes exist, whose specific rules are established through sector-specific collective bargaining agreements. These regimes deviate sometimes quite substantially from the standard regime - most notably in terms of generally looser career and claiming age conditions,

\footnotetext{
${ }^{91}$ Each year of career must be constituted of at least 104 days of FTE.

${ }^{92}$ See Royal decree of the $20^{\text {st }}$ of January 2015 - Arrêté royal modifiant l'article 64 de l'arrêté royal du 21 décembre 1967 portant règlement général du régime de pension de retraite et de survie des travailleurs salariés, M.B. 21 January 2015.

${ }^{93}$ See the appendix for a list of the earnings test for the year 2020 (pivotal index 147.31).

${ }^{94}$ Sections 2.3, 2.4, 2.5 and 2.7 essentially correspond to a more in-depth analysis of the programs presented in Fraikin, Jousten and Lefebvre (2020).

${ }_{95}$ Another early retirement programs co-existed with the conventional early retirement scheme, the legal early retirement. Contrary to the conventional early retirement, the worker had the legal right to benefit from this program and the supplement to unemployment benefits was paid by the State instead of the company. Because of its generous nature, the program quickly became too expensive and was discontinued in 1982.

${ }^{96}$ See the Collective Labor Agreement 17 of the $19^{\text {th }}$ of December 1974, implemented by the Royal Decree of the $16^{\text {th }}$ of January 1975 - Arrêté royal rendant obligatoire la convention collective de travail $n^{\circ} 17$, M.B. 31 January 1975.

${ }^{97}$ See the Royal Decree of the 19th of February 1975 - Arrêté Royal concernant le droit des travailleurs âgés aux allocations de chômage, M.B. 21 March 1975.
} 
with an eligibility age as low as 50 in some cases. Moreover, special rules prevail for workers in companies in distress, working in dangerous professions or with long careers. This broadening of eligibility rules led to the creation of a large pool of early retirees who benefitted from a generous replacement income, for sometimes more than 10 years, which was financially unsustainable in the long run (Gieselink, Stevens and Van Buggenhout, 2002). In the early eighties, it became clear that the program had become too expensive following its growing success and did not lead to the expected result of diminishing the unemployment rates of younger workers. Consequently, the government initiated a wave of reforms that tightened the eligibility criteria of the program (detailed in the section below).

From $1986^{98}$ until 2011, employers were required to replace a conventional early retiree below age $60^{99}$ by a new worker hired off the unemployment insurance rolls. However, the one-for-one worker substitution rules contained many exceptions, also contributing to a substantially lower aggregate replacement effect at the macro level. In 2011, the minimum duration of the replacement obligation is set at 36 months. In $2015^{107}$, the age criteria of 60 is replaced by 62 .

Until 2015, conventional early retirement beneficiaries were fully exempted from job search and did not have to be available for the labor market. Since 2015, a new notion of "adapted availability" has been introduced in the applicable rules, essentially making numerous early retirees subject to an availability condition up until the statutory

\footnotetext{
${ }^{98}$ See Royal Decree of the 30 $30^{\text {st }}$ of August 1985 - Arrêté Royal portant nouvelle réglementation de l'octroi d'allocations de chômage en cas de prépension conventionnelle, M.B. 7 December 1985.

${ }_{99}$ No replacement obligation exists for early retirees aged more than 60.
} 
eligibility age - though it remains less stringent than for regular unemployment benefits. ${ }^{100}$

\subsubsection{BENEFIT CALCULATION RULES}

Upon satisfying lay-off, career and claiming age conditions, conventional early retirees are entitled to a two-tiered early retirement benefit: an unemployment insurance benefit combined with a company supplement paid by the former employer. Conventional early retirement benefits are payable until reaching the old-age pension statutory retirement age - upon which beneficiaries are automatically rolled over into the old-age pension system. The unemployment insurance part of the benefit consists of a slightly more generous version of the standard unemployment insurance benefit- with the most noticeable deviation being a lack of a degressive function for benefit duration and a more favorable treatment for individuals not living alone. The company supplement corresponds to half the difference between the last net wage and the unemployment benefit.

Until 2011, conventional early retirement benefits were included in the pension calculation at the same rate as the last wage received. Starting from $2012^{101}$, the conventional early retirement benefits that are credited the old-age pension benefits calculation are limited to the legal minimum wage amount for individuals below the age of 58 (except in the case of companies with financial constraints or in restructuration).

\footnotetext{
${ }^{100}$ More recently in 2015, the name of the scheme has been adapted to unemployment scheme with company supplement - mirroring this conceptual shift.

${ }^{101}$ See Royal decree of the $27^{\text {th }}$ of February 2013 - Arrêté royal portant exécution de l'article 122 de la loi du 28 décembre 2011 portant des dispositions diverses et modifiant diverses dispositions en matière de périodes assimilées, M.B. 8 March 2013.
} 


\subsubsection{ELIGIBILITY AGES AND CAREER REQUIREMENTS}

The eligibility age of the conventional early retirement scheme, which is generally lower than the early eligibility age of the old-age pension scheme, has undergone substantial reforms. The conventional early retirement scheme was initially designed to give workers the opportunity to retire 5 years before the statutory eligibility age of the old-age pension system (60 for men and 55 for women). Several collective bargaining agreements rapidly lowered the effectively applicable eligibility ages and other eligibility conditions. As a result, the Belgian reality in the area of conventional early retirement has been characterized from the early days by the coexistence of regimes with different career requirements, minimum ages, etc. Though legislation in the early 1980's tried to harmonize and put constraints, numerous exceptions persist with respect to the general rules.

In response to the success of the program and thus the growing proportion of older workers retiring through the conventional early retirement scheme, the eligibility criteria were tightened over the years. In 1984 ${ }^{102}$, the eligibility age for the conventional early retirement is set at 55 . In $1986^{103}$, the eligibility age increases to 57 and career length requirements are introduced: at least 5 years in the same company, 10 years in the same industry and 20 years of career as a wage earner. In 1987104, the eligibility age increases to 58. In $1990^{105}$, career length requirements are increased to 38 years for workers aged 55, 25 years of career for workers aged 56 to 59 and 20 years for workers aged more than 59 .

\footnotetext{
102 See Royal Decree of the $18^{\text {th }}$ of July 1983

103 See Royal Decree of the $20^{\text {st }}$ of August 1986 - Arrêté royal relative à l'octroi d'allocations de chômage en cas de prépension conventionnelle, M.B. 10 September 1986.

104 See Royal Decree of the 22 ${ }^{\text {nd }}$ of October 1987 - Arrêté Royal portant modification de l'A.R. du 20 août 1986 relatif à l'ctroi d'allocations de chômage en cas de prépension conventionnelle, M.B. 17 November 1987.

105 See Royal Decree of the $16^{\text {th }}$ of November 1990 - Arrêté Royal relative à l'octroi d'allocations de chômage en cas de prépension conventionnelle, M.B. 23 November 1990.
} 
Moreover, assimilated periods can now be included in the eligibility criteria of the conventional early retirement scheme. In $2008^{106}$, the minimum age increases to 60 with at least 30 years of career for men and 26 for women. However, the Collective Bargaining Agreements that were in place since 1986 allowed access before 60 and remained valid until (at most) 2014. Therefore, the conventional early retirement program remained accessible in some industries at age 58 with a career of at least 35 (30) years for men (women), at age 56 with a career of at least 40 years (until 2011) or age 57 with a career of at least 40 years (until 2014). Starting from 2007106, the career length requirements have gradually increased over the years. At age 58, the career length requirements for men increased to 37 in 2010, 38 in 2012 and 40 in 2015. The career requirement for women aged 58 increased to 33 in 2010, 35 in 2012, 38 in 2014, 39 in 2016 and 40 in 2017. At age 60, the career length requirement for men increased to 35 in 2012, 36 in 2013, 37 in 2014 and 40 in 2015. The career requirement for women aged 60 increased to 28 in 2012, 31 in 2015, 32 in 2016, 33 in 2017, 34 in 2018, 35 in 2019, 36 in 2020, 37 in 2021, 38 in 2022, 39 in 2023 and 40 in 2024. Therefore, by 2024 the gender disparity in eligibility criteria will have disappeared. Finally, in $2015^{107}$, the minimum age is raised from 60 to 62 but the program remains accessible at age 58 (until 2017) or age 59 with a career of at least 40 years. Figure 2.10 summarizes the evolution of the career length requirement at different eligibility ages.

\footnotetext{
106 See Royal Decree of the $3^{\text {rd }}$ of May 2007 - Arrêté Royal fixant la prépension conventionnelle dans le cadre du Pacte de Solidarité entre les générations, M.B. 8 June 2007.

107 See Royal Decree of the 23rd of April 2013 - Arrêté royal modifiant l'arrêté royal du 3 mai 2007 fixant le régime de chômage avec complément d'entreprise, M.B. 2 October 2013.
} 
Figure 2.10: Historical evolution of career length requirement for the standard conventional early retirement scheme - by eligibility ages and gender
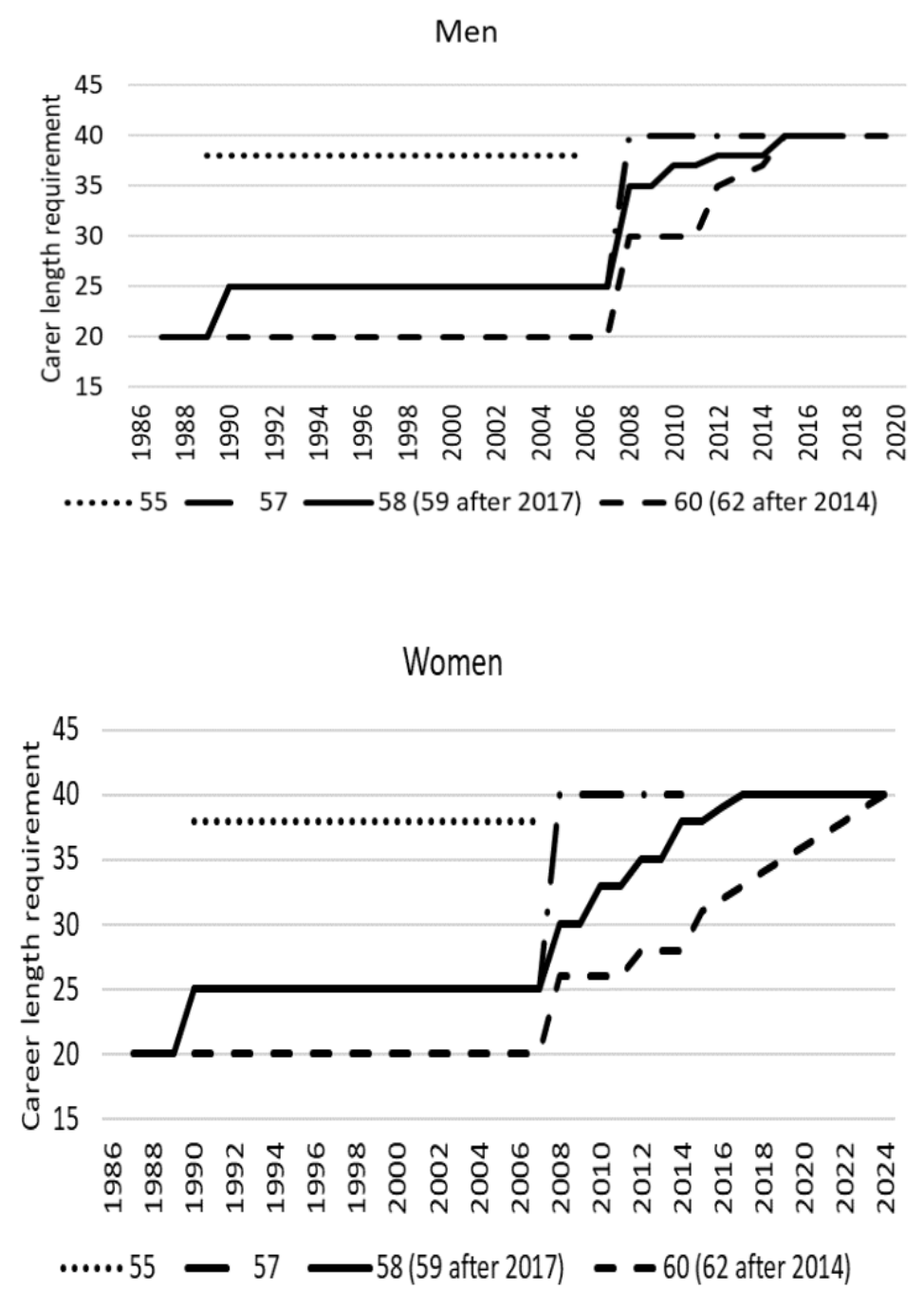

Source: Claes (2012), Gieselink, Stevens and Van Buggenhou (2002) 


\subsection{UNEMPLOYMENT INSURANCE}

Next to the old-age pension scheme and the conventional early retirement, the unemployment insurance system is an important pathway to retirement. Unemployment benefits are available to laid-off workers (for reasons other than serious misconduct) of all ages under the condition of having paid contributions during at least 12 months in employment or having been in assimilated status (sickness, etc.) in the last 18 months. Under the regular system, individuals must be available for the job market and actively look for employment.

The amount of unemployment benefits is based on the last gross wage ${ }^{108}$, the family situation, the number of contribution years and the duration of the unemployment spell. At the beginning of the unemployment spell, the benefits generally represent 60 percent of the last gross wage for unemployed with family dependents, 55 percent for single unemployed and 40 percent for individuals that are sharing their household with others (effective cohabitation). Unemployment benefits are subject to a degressive mechanism whereby as the unemployment duration increases, they are essentially bringing them down to a flat rate amount irrespectively of prior earnings. There are minimum and maximum unemployment benefits that also vary by household status and also decrease for long unemployment duration. Benefits are not time-limited and can in principle be payable until reaching the old-age pension statutory eligibility age - upon which beneficiaries are automatically rolled over into the old-age pension system ${ }^{109}$.

\footnotetext{
${ }^{108}$ The notion of last gross wage differs from the one used in the old-age pension scheme: it is the last gross wage received during the individual's last continuous employment of at least 4 weeks, subject to a set of floor and ceilings.

109 See Royal Decree of the $25^{\text {th }}$ of November 1991 - Arrêté royal portant réglementation du chômage, M.B. 31 December 1991.
} 
Until 2011, periods spent in the unemployment insurance scheme were credited in the old-age pension calculation at the rate of the last wage received for their full duration. Since $2012^{110}$, the credited amount of unemployment benefits that enters the pension benefits calculation is limited to the minimum annual credit starting from the ' 3 rd period' of unemployment (between 12 and 36 months). ${ }^{11}$

\subsubsection{OLD-AGE UNEMPLOYMENT}

The old age unemployment program is introduced in $1984^{112}$ with the objective of providing a stable and decent income to older unemployed workers who are highly unlikely to find a job and are thus likely to remain unemployed for an abnormally long period (Gieselink, Stevens and Van Buggenhout, 2002). The scheme includes a waiver of job search and a seniority supplement.

\subsubsection{WAIVER OF JOB SEARCH}

The waiver of job search is available for workers above the age of 55 or 50 for workers who can prove a permanent work incapacity. In 1995113, the permanent incapacity requirement is dropped for workers aged 50 to 55 and the waiver of job search becomes available for everyone above age 50. Following this reform, the number of beneficiaries of the old-age unemployment scheme skyrocketed and even exceeded the number of beneficiaries of the conventional early retirement scheme (Gieselink, Stevens and Van

\footnotetext{
110 See Royal decree of the $27^{\text {th }}$ of February 2013 - Arrêté royal portant exécution de l'article 122 de la loi du 28 décembre 2011 portant des dispositions diverses et modifiant diverses dispositions en matière de périodes assimilées, M.B. 8 March 2013.

111 The $3^{\text {rd }}$ period of unemployment benefits starts after $[(12+2+(2 \times$ years employed $)]$ months spent on unemployment benefits and corresponds to the start of lump sum unemployment benefits.

112 See Royal Decree of the 29 $9^{\text {th }}$ of December 1984 - Arrêté royal relatif à l'emploi et au chômage, M.B. 18 January 1985.

113 See the Royal Decree of the 22nd of November 1995 - Arrêté Royal modifiant l'arrêté royal du 25 novembre 1911 portant réglementation du chômage dans le cadre du plan pluriannuel pour l'emploi, M.B. 8 December 1995.
} 
Buggenhout, 2002). Indeed, an increasing number of companies made use of the old-age unemployment scheme to lay off their older workers to decrease their operating costs, a practice labelled 'Canada Dry early retirement'. Several factors are at the root of this practice: the relatively flexible eligibility conditions of the old-age unemployment program, the absence of an obligation to replace the laid off worker with an unemployed worker and lower social contributions. In order to decrease the use of the Canada Dry early retirement mechanism, the minimum age for the waiver of job search is raised to 58 in $2004^{114}$ and to 60 in $2013^{115}$. The waiver of job search remains accessible below age 60 for workers with a career of at least 38 years.

\subsubsection{SENIORITY SUPPLEMENT}

The seniority supplement is introduced in $1989^{116}$. It is granted to workers aged more than 50 starting from their second year of unemployment if they have a wage earner career of at least 20 years. The supplement was introduced to replace the adaptation complement, paid to unemployed workers during their first year of unemployment (Gieselink, Stevens and Van Buggenhout, 2002). The amount of the seniority supplement is adjusted for inflation with the pivotal index and is differentiated between individuals according to age and household status (legal cohabitants with dependent family, isolated or legal cohabitants without family dependent). ${ }^{117}$ Seniority supplements are subject to a set of

\footnotetext{
114 See Royal Decree of the 27th of May 2002 - Arrêté Royal modifiant l'arrêté Royal du 25 novembre 1991 portant réglementation du chômage dans le cadre de l'augmentation du taux d'emploi des travailleurs âgés, M.B. 11 June 2002.

115 See Royal Decree of the $10^{\text {th }}$ of November 2012 - Arrêté royal modifiant les articles 59bis et 89 de l' arrêté royal du 25 novembre 1991 portant réglementation du chômage et modifiant l'article 10 de l' arrêté royal du 4 juillet 2004 portant modification de la réglementation du chômage à l'égard des chômeurs complets qui doivent rechercher activement un emploi, M.B. 22 November 2012.

116 See the Royal Decree of the $13^{\text {th }}$ of January 1989 - Arrêté Royal relative à l'octroi d'un complement d'ancienneté aux chômeurs âgés, M.B. 19 January 1989.

117 The seniority supplement for legal cohabitants with dependent family is a daily lump-sum of 3.54 euros (pivotal index 147.31), the supplement for isolated individuals is equal to 5 percent of the daily limited
} 
floor and ceilings. ${ }^{118}$ At present, no seniority supplement subsists: the minimum age was raised to 55 in $2013^{115}$ before being completely dropped in $2015 .{ }^{119}$

\subsection{DISABILITY INSURANCE}

The Disability Insurance (DI) system is the main program targeted at people withdrawing from the labor market for health-related reasons - though it has become an increasingly relevant early retirement route considering ever-tighter eligibility and benefit criteria for the conventional early retirement and unemployment systems (Jousten, Lefebvre and Perelman, 2011). The disability insurance provides disabled workers with a replacement income if they show evidence of a minimum of 66 percent loss of their earning capacity for at least 12 consecutive months. During the first 12 months of work incapacity, disabled workers are eligible for sickness benefits payable by the employer and the sick pay insurance. Both the sickness and the disability benefits enter the old-age pension formula at the same rate as the last gross wage received before the start of the incapacity period.

Sickness benefits take the form of a 60 percent replacement rate of the last gross wage received. ${ }^{122}$ Starting from the seventh month sickness, the household situation ${ }^{120}$ and the

\footnotetext{
wage and the supplement for legal cohabitants without family dependent is equal to 10 percent of the daily limited wage before age 58 and 15 percent after age 57.

118 In 2020 (pivotal index 147.31), the minimum daily amount of unemployment benefits and the seniority supplement is 54.1 in the case of dependent family, 48.52 for isolated individuals, 39.42 for legal cohabitants without family dependent and below age 58 and 43.3 for legal cohabitants without family dependent above age 57. The corresponding maximum daily amount is $60.43,55.37,46.14$ and 50.75, respectively.

119 See Royal Decree of the $1^{\text {st }}$ of June 2015 - Arrêté royal modifiant l'article 89 de l'arrêté royal du 25 novembre 1991 portant réglementation du chômage et insérant un article 89/1 dans le même arrêté royal, M.B. 10 June 2015. ${ }^{120}$ The definition of dependent differs from the definition of one-earner households in the old-age pension calculation. More information on the National disability and sickness insurance institute website (See 'Votre situation familiale', https://www.inami.fgov.be/fr/themes/incapacite-travail/Pages/situationfamiliale.aspx).
} 
status of regular worker determines the guaranteed minimum sickness benefits. ${ }^{121}$ The disability benefits are calculated as 55 percent of the worker's last gross income. ${ }^{122}$ The replacement rate of the disability benefits increases to 65 percent if the individual is legally cohabitating with a partner and has dependent family-members ${ }^{120}$ and decreases to 40 percent for singles and legally cohabitating individuals without family dependent. Both sickness and disability benefits are subject to a set of floors and ceilings. ${ }^{123}$

To be eligible for the benefit, the worker must meet a series of conditions. First, there is a condition in terms of the loss of earnings capacity in the usual job. In order to be eligible, the worker must suffer from a loss of earnings capacity of 66 percent over a period of at least twelve months. After first benefit entitlement, continued eligibility is periodically validated using medical and administrative controls. Second, workers also must satisfy minimum contributory requirements to qualify for benefits. The coverage under the system prior to the onset of the disability must be assured for at least two quarters, combined with at least 120 days of actual or assimilated work (or 400 hours for -part-time workers) before the covered event occurs.

As for the unemployment and conventional early retirement systems, disability benefits are payable up to the statutory eligibility age. Contrary to other social insurance

\footnotetext{
${ }^{121}$ In 2020 (pivotal index 147.31), the daily minimum sickness pay is 62.08 for a regular worker with a dependent spouse or a dependent child, 49.68 for a regular worker who is isolated, 42.6 for a legally cohabitating regular worker, 49.84 for an irregular worker with dependents and 36.88 for an irregular worker without dependent. The benefits are paid on the basis of a 6-days week. (see 'Montant minimal de votre indemnité à partir du $7 \mathrm{e}$ mois d'incapacité de travail', https://www.inami.fgov.be/fr/themes/incapacite-travail/montants/salaries-chomeurs/Pages/indemnite-7emois-min-salarie.aspx).

122 The last gross wage taken into account in the sickness and disability benefits calculation is the gross wage of the last day of the second quarter of the year before the inactivity period.

123 See the appendix for a list of ceilings for sickness or disability benefits.
} 
replacement income programs, periods of benefit receipt continue to fully count toward the build-up of pension rights at the value of the last observed wage to this date.

No major reforms have affected the disability benefits calculation scheme over the last decades.

\subsection{TIME CREDIT}

The old-age time credit scheme is created in $2002^{124}$ for workers aged 50 and over ${ }^{125}$ and allows for part time work and the receipt of a partial replacement income paid by the unemployment insurance system. Two types of time credit plans exist: one that allows for a work interruption of one-half of total work time and the other that allows for a work interruption of one-fifth of total work time. While the latter is rather generally accessible as of age 55, the former requires additional eligibility conditions including a minimum total number of career years. The time credit benefit consists in a lump-sum amount that varies according to type of work interruption (one half or one-fifth), and the household situation (isolated or legal cohabitants, with or without dependent children). ${ }^{126}$

In view of its growing success, the eligibility criteria of the time credit program were tightened over the years. In $2012^{127}$, the minimum eligibility age increases to 55 with at least 25 years of career (assimilated periods included) and 24 continued months working

\footnotetext{
124 See Royal Decree of the 12 $2^{\text {th }}$ of December 2001 - Arrêté royal pris en exécution du chapitre IV de la loi du 10 août 2001 relative à la conciliation entre l'emploi et la qualité de vie concernant le système du crédit-temps, la diminution de carrière et la réduction des prestations de travail à mi-temps, M.B. 18 December 2001.

${ }^{125}$ Derogatory eligibility conditions exist for workers in companies in distress, dangerous professions or with long careers.

126 See the appendix for the historical evolution of time credit benefits.

127 See Royal Decree of the $28^{\text {th }}$ of December 2011 - Arrêté Royal modifiant l'arrêté royal du 12 décembre 2001 pris en exécution du chapitre IV de la loi du 10 août 2001 relative à la conciliation entre l'emploi et la qualité de vie concernant le système du crédit-temps, la diminution de carrière et la réduction des prestations de travail à mitemps, M.B. 30 December 2011.
} 
for the same employer. The minimum age for the receipt of the allowance gradually increased from 55 to $60^{128}$ from 2016 to $2019 .{ }^{129}$ However, it is still possible to benefit from time credit benefits at age 57 in the case of one-half work interruption and at age 55 for one-fifth work interruption if the worker has at least 35 years of career. Since 2003130, periods spent receiving time credit benefits are included in the pension benefit calculation as assimilated periods. Starting from 2012 ${ }^{131}$, only one year spent in the time credit program can be included as an assimilated period in the old-age pension benefits calculation.

\subsection{INCOME TAXES AND SOCIAL SECURITY CONTRIBUTIONS}

In Belgium, both the earnings and social security benefits are subject to income taxes and mandatory social contributions. Employer social contributions amount to 24.92 percent of the gross wage. Personal/employee social contributions include a 13.07 percent employee contribution on wage income, a 3.5 percent contribution on disability benefits and a 6.5 percent contribution on conventional early retirement and unemployment benefits. While

\footnotetext{
${ }^{128}$ It is still possible to benefit from time credit benefits if there exist special rules in a Collective Bargaining Agreement within the sector (age 55 for one-fifth work reduction or age 57 for one-half work reduction).

129 See Royal Decree of the 30st of December 2014 - Arrêté royal modifiant l'arrêté royal du 12 décembre 2001 pris en exécution du chapitre IV de la loi du 10 août 2001 relative à la conciliation entre l'emploi et la qualité de vie concernant le système du crédit-temps, la diminution de carrière et la réduction des prestations de travail à mitemps, M.B. 31 December 2014.

130 See Royal Decree of the $21^{\text {st }}$ of January 2003 - Arrêté royal modifiant l'arrêté royal $n^{\circ} 50$ du 24 octobre 1967 relatif à la pension de retraite et de survie des travailleurs salariés, et l'arrêté royal du 21 décembre 1967 portant règlement général du régime de pension de retraite et de survie des travailleurs salariés, M.B. 3 February 2003. ${ }^{131}$ See Royal decree of the 27th of February 2013 - Arrêté royal portant exécution de l'article 122 de la loi du 28 décembre 2011 portant des dispositions diverses et modifiant diverses dispositions en matière de périodes assimilées, M.B. 8 March 2013.
} 
social security benefits are all capped by means of program-specific ceilings, no contribution ceilings are applicable.

The Belgian personal income tax (PIT) applies to wage, old-age pension and other replacement income. The PIT liability is calculated using a strongly progressive rate structure with marginal tax rates from 0 to 50 percent from 2003 onwards. Before 2003, the tax rates were modified substantially over the years and could go as high as 70.8 percent in 1988. Tax exempted income amounts vary according to the marital status and the number of dependent children of the taxpayer.

There exist two types of tax deductions for social security benefits: a first one that is linked to the household situation and a second one that cancels the tax if the social security benefits is below a certain threshold. ${ }^{132}$ The combination of these deductions leads to an important fiscal exoneration for social security benefits.

The first type of tax deduction is a set of ad hoc tax deductions for the various categories of replacement income. These deductions are effectively granting these types of income a preferential treatment as compared to wages and other types of income and are effectively excluding a substantial share of these benefits from taxation. The deduction amount is similar for the old-age pension system and unemployment but differs for the conventional early retirement scheme and disability benefits system. At first higher, the tax deduction for conventional early retirement benefits decreased in 1999 to equal the level of the deduction for the old-age pension system and unemployment insurance scheme. Since then, the deduction for the disability benefits is the highest (see figure 2.11). The differentiated deduction amount for one-earner households is abolished in 2004 for the

132 See the appendix for a list of the tax deductions for replacement income. 
old-age pension and disability insurance schemes, in 2005 for the conventional early retirement scheme and in 2009 for the old-age unemployment scheme.

The second tax deduction reduces the total tax amounts to zero if a person's income is exclusively composed of old-age pension or other replacement benefits below a given threshold, which has been increasing steadily over the years (see figure 2.12). The income thresholds for the second tax deduction are similar for old-age pension and conventional early retirement benefits (and unemployment benefits until 1988). The income threshold for unemployment benefits increased to the level of the threshold for disability benefits in 1989. Today, the income thresholds for the second social security tax deduction are the highest for disability and unemployment benefits.

Figure 2.11: Evolution of the first deduction for replacement income

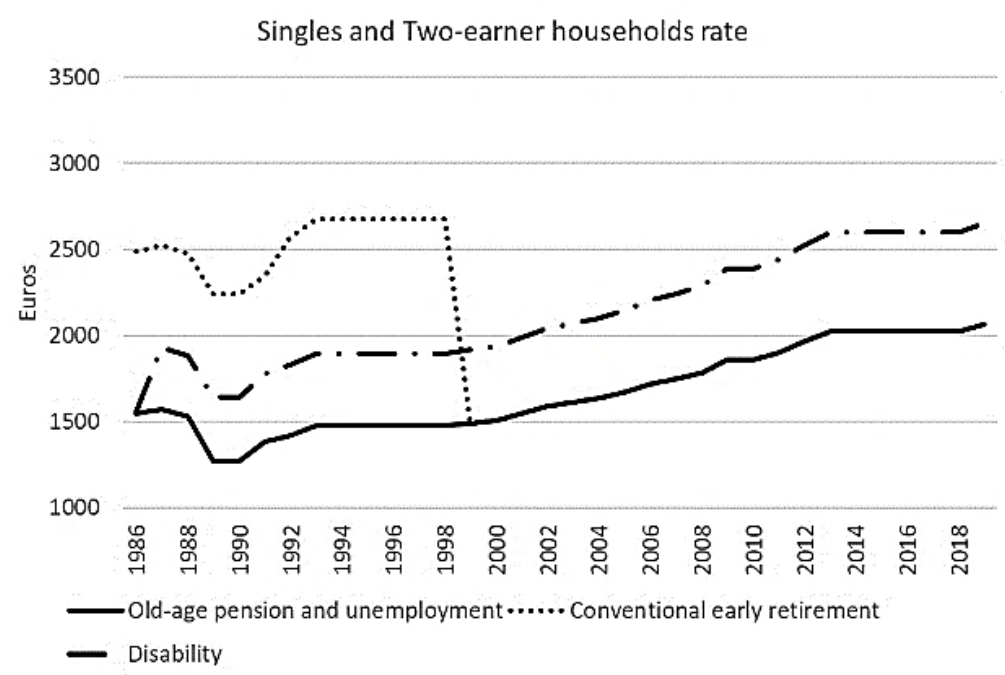




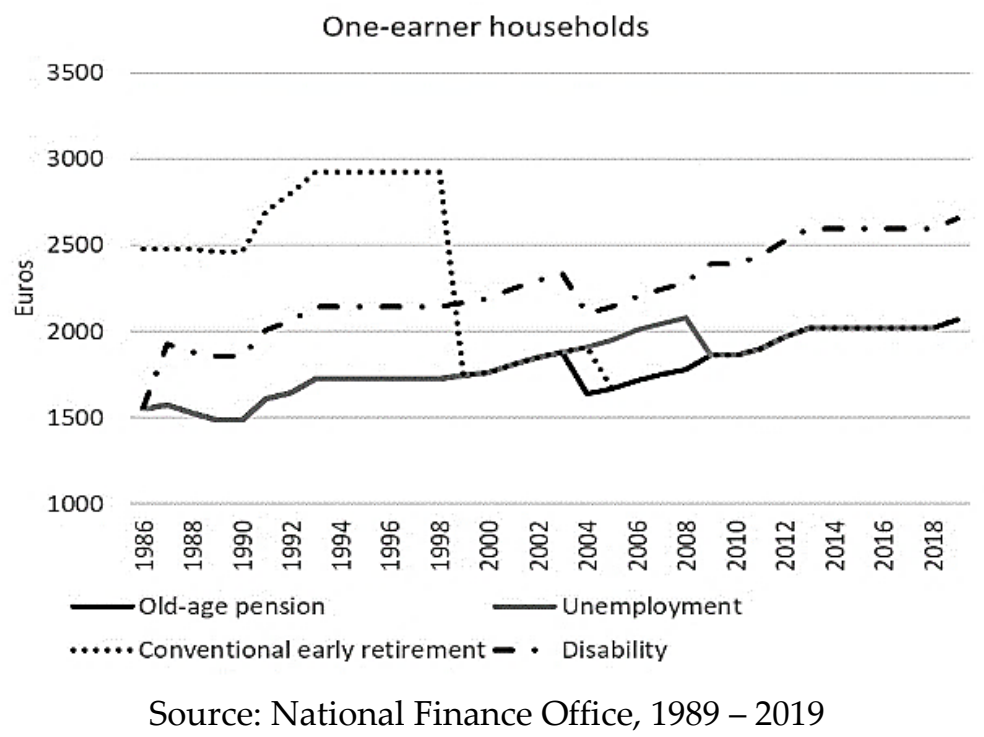

Figure 2.12: Evolution of the second deduction for replacement income

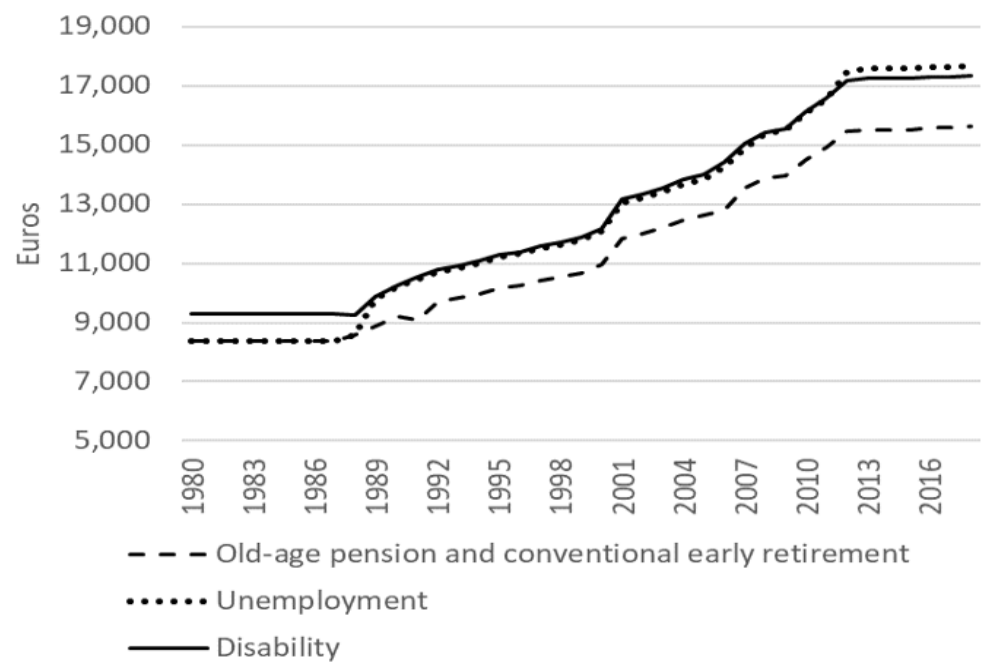

Source: National Finance Office, 1989 - 2019

Additional contributions exist for the old-age pension scheme. The solidarity contribution essentially taxes beneficiaries of higher pensions and varies between 0 to 2 percent of the gross old-age pension amount of the first and the second pillar (see table 2.5) and vary according to the household situation. The Health and Disability contribution is introduced in $1994^{133}$. It amounts to 3.55 percent of the gross old-age pension amount,

${ }^{133}$ See Law of the 30 st of March 1994 - Loi portant des dispositions sociales, M.B. 31 March 1994. 
vary according to whether the pensioner has dependent children and is only applicable for pensions that are above a certain threshold (see table 2.6).

Table 2.5: Solidarity contribution rate (2020 rules)

\begin{tabular}{|c|c|c|c|}
\hline \multicolumn{2}{|c|}{ Two-earner and single households } & \multicolumn{2}{|c|}{ One-earner households } \\
\hline $\begin{array}{l}\text { Old-age pension } \\
\text { benefits bracket }\end{array}$ & $\begin{array}{l}\text { Solidarity contribution } \\
\text { amount (2020 euros) }\end{array}$ & $\begin{array}{l}\text { Old-age pension } \\
\text { benefits bracket }\end{array}$ & $\begin{array}{c}\text { Solidarity contribution } \\
\text { amount (2020 euros) }\end{array}$ \\
\hline 0.01 and $2646.32 €$ & 0 & 0.01 and $3059.47 €$ & 0 \\
\hline $\begin{array}{l}2646.33 \text { and } \\
272815 €\end{array}$ & $(x-2646.32) \times 0.5$ & $\begin{array}{l}3059.48 \text { and } \\
315407 €\end{array}$ & $(x-3154.07) \times 0.5$ \\
\hline $\begin{array}{l}2728.16 \text { and } \\
2931.02 €\end{array}$ & $x \times 0.015$ & $\begin{array}{l}3154.08 \text { and } \\
3353.03 €\end{array}$ & $x \times 0.015$ \\
\hline 2931.03 and & $(x-2931.02) \times 0.5$ & 3353.04 and & $(x-3353.04) \times 0.5$ \\
\hline $2961.55 €$ & +43.97 & $3387.95 €$ & +50.3 \\
\hline $\begin{array}{l}\text { Superior to } 2961.55 \\
€\end{array}$ & $x \times 0.012$ & $\begin{array}{l}\text { Superior to } 3387.95 \\
€\end{array}$ & $x \times 0.02$ \\
\hline
\end{tabular}

Note: $\mathrm{x}=$ gross old-age pension benefits amount

Source: National Pension Office at www.sfpd.fgov.be

Table 2.6: Health and Disability contribution rate (2020 rules)

\begin{tabular}{|c|c|c|c|}
\hline \multicolumn{2}{|c|}{ Without dependent children } & \multicolumn{2}{|c|}{ With dependent children } \\
\hline $\begin{array}{l}\text { Old-age pension } \\
\text { benefits bracket }\end{array}$ & $\begin{array}{l}\text { Health and disability } \\
\text { contribution rate }\end{array}$ & $\begin{array}{l}\text { Old-age pension } \\
\text { benefits bracket }\end{array}$ & $\begin{array}{c}\text { Health and } \\
\text { disability } \\
\text { contribution rate }\end{array}$ \\
\hline Inferior to $1560.97 €$ & 0 & Inferior to $1849.97 €$ & 0 \\
\hline 1560.97 and $1618.4 €$ & $(x-1560.97)$ & 1849.97 and $1918.04 €$ & $(x-1849.97)$ \\
\hline Superior to $1618.4 €$ & $x \times 0.0355$ & Superior to $1918.04 €$ & $x \times 0.0355$ \\
\hline
\end{tabular}

Note: $\mathrm{x}=$ gross old-age pension benefits amount Source: National Pension Office at www.sfpd.fgov.be 


\subsection{CONCLUSION}

This chapter presents a thorough review of the existing social security regulations and their evolution over the eighties until today. In particular, we describe the evolution of the benefit calculation parameters and eligibility rules of the four main labor force exit pathways for older Belgian workers: the old-age pension, the conventional early retirement, the unemployment insurance and the disability insurance. Additionally, we take a close look at the time credit scheme for older workers.

We demonstrate the convolution of the Belgian social security system and the high potential for intertwined effects of its eligibility and calculation rules between different programs. Such complexity creates an opaque and incoherent system that makes it increasingly challenging for citizens and policy makers to gain an understanding of how benefits are calculated. It is clear that complex social security rules are inevitable in today's society as they reflect the historical evolution the Belgian labor market regulation and organization and serve to capture the specificities of different types of workers. However, the lack of cautious ex-ante and ex-post analysis of social security reforms creates an environment in which the intertwined effects between various social security programs are not fully understood, which might sometimes lead to reforms working in opposite directions.

This work is relevant for policy makers and researchers alike. On the one hand, it is crucial that decision makers have a good understanding of the current social security system in order to pave the way for the necessary sustainability reforms that lie ahead. On the other hand, even the tiniest detail in pension benefits calculation may well have a significant role to play in the computation of financial retirement incentives in the analysis of retirement behavior. Simplifying the calculation of old-age pension benefits might lead 
to misleading theoretical benefits, and thus erroneous financial incentives measures and misinforming conclusions. Such a detailed review of the regulation of social security programs that are relevant for the analysis of the retirement decision is still missing upto-date, although some studies exist for some programs separately. Further research on the potential intertwined effects of the various reforms presented in this chapter and their implications for the retirement behavior would be highly valuable and beneficial from a public policy point of view. 


\section{REFERENCES}

Berghman, J. and Peeters, H. (2012). Les trois piliers du paysage belge des pensions de retraite. Aperçu et défis. Revue belge de sécurité sociale, 54(1), 1-54. *

Bollen, N. (2017, February 18). Gunstregime voor landingsbaan na lange loopbaan verdwijnt. De Tijd, 6.*

Bureau fédéral du Plan Economic Policy Committee's Ageing Working Group. (2017, November). Belgium: Country Fiche 2017. Bruxelles : Bureau fédéral du Plan. *

Canazza, C. and Mary, G. (2012). La sécurisation du parcours professionnel des chômeurs âgés. Revue belge de Sécurité Sociale, 54, 55-124. *

Claes, T. (2012). La prépension Conventionnelle (1974-2012). Courier hedomadaire du CRISP, 29(2154-2155), 5-94. *

Colin, C. and Metten, C. (2003). Impact des différents aléas de carrière sur les retraites: inactivité, chômage, travail à temps partiel et préretraite. La Documentation française "Retraite et société", 3(40), 21-51. *

Pension Reform Committee 2020-2040. (2014). Un Contrat Social Performant et Fiable. Bruxelles: SPF Sécurité Sociale.

Conseil Supérieur des Finances. (2012). Avis du Comité d'étude sur le vieillissement: Évaluation du bonus pension. Bruxelles : Bureau fédéral du Plan.

Corbus. (n.d.). Adaptation de la réglementation du chômage à l'égard des chômeurs âgés. Retrieved from Corbus: https://www.corbus.be/documents/newsitems/kl1609221-beschikbaarheidsbeginsel-opgetrokken-tot-60-jaar-.xml?lang=fr * 
De la Croix, D. and Pestieau, P. (2007/1). Réformer le système des retraites belges. Reflets et perspectives de la vie économique, tome xlvi(1), 113-127. *

De vil, G., Fasquelle, N., Joyeux, C., Scholtus, B., and Weemaes, S. (2015, September). Analyse des effets de la réforme de spension et du chômage avec complément d'entreprise. Bureau fédéral du Plan Centre d'expertise des pension REP_CEP1_11080.*

Dekkers, G., Desmet, R., and Van den Bosch, K. (2018). Revalorisation réelle des salaires dans le calcul de pension des travailleurs salariés. Bruxelles: Bureau fédéral du Plan.

Dellis, A., Desmet, R., Jousten, A., and Perelman, S. (2004). Analyse de la cessation d'activité en Belgique. Revue française d'économie, 18(1), 99-132.*

European Commission (DG-EMPL). (2018). The 2018 Pension Adequacy Report: Current and future income adequacy in old age in the EU. country profiles. Luxembourg: Publications Office of the European Union.

Fasquelle, N. and Weemaes, S. (1997, November). Perspectives financières de la Sécurité sociale à l'horizon 2050. Bureau fédéral du Plan Planning Paper 83. *

Feestjens, M. (1997, November). La réforme des pensions. Bureau fédéral du Plan Planning Paper 82.*

Fraikin, A.L., Jousten, A., and Lefèbvre, M. (2020). Social Security Incentives for Retirement in Belgium: An analysis of four decades of change. In Börsch-Supan, Coile, Social security around the world. Chicago: University of Chicago Press and NBER.

Fusulier, B., Moulaert, T., Tremblay, D., and Larivière, M. (2009). Travailler plus longtemps !? L'aménagement des fins de carrière en Belgique et au Québec. November. * 
Gieselink, G., Stevens, Y., and Van Buggenhout, B. (2002, 4th quarter). Départ anticipé des travailleurs âgés en Belgique. (S. p. sociale, Ed.) Revue belge de Sécurité Sociale, 579-618. *

Grolaux, L. (2016). Le régime de chômage avec complément d'entreprise. Mémoire Master en droit. Louvain-La-Neuve: Faculté de Droit et de criminologie Université Catholique de Louvain. *

Leduc, A. (2011). Analyses et études 2011. Retrieved from Cfs ep: http://ep.cfsasbl.be/Lesysteme-des-pensions-en-Belgique-solidarite-ou-responsabilite-individuelle

Lefebvre, M., Orsini, K., and Paszukiewicz, A. (2009/3). La retraite anticipée des salariés en Belgique. Revue économique, 60, 777-785. *

Nevejan, H. and Van Camp, G. (4th quarter 2013). La dégréssnivité renforcée des allocations de chômage belges: un premier bilan. Revue belge de sécurité sociale, 497-562. *

OECD. (2017). Pensions at a Glance 2017: OECD and G20 Indicators. Paris: OECD Publishing.

ONEM. (2010). 75 ans de l'ONEM Un regard sur le passé, le présent et le futur. Bruxelles: ONEM. *

Peeters, H. and Van Camp, G. (2016). Het belang en de samenstelling van gelijkgestelde periodes in de drie pensioenstelsels Een stand van zaken. Bureau fédéral du Plan Centre d'expertise des pensions REP_CEP4_11186. *

Peeters, H. and Van Oorschot, W. (2016). The devil is in the detail. Delving into Belgian pension adequacy. Faculteit sociale Wetenschappen KU Leuven. 
Pestieau, P. and Stijns, J. (1997). Social Security and Retirement in Belgium. NBER Working paper series 6169.*

Poulain, J., Bergeret, P., and Noel, F. (2018). Calcul de la pension de retraite droits dérivés activités après la pension. Séance d'information sur les pensions "Région Wallonne octobre 2018". Service fédéral des Pensions. *

Sanderson, J. (2015). Vieillissement de la population et retraites en Belgique, 19ème20ème siècles. (J. D. v.z.w., Ed.) Revue Belge d'Histoire Contemporaine, 2015(4), 1-15.

Secrétariat social UCM. (2012, Avril). Chômage avec complément d'entreprise situation depuis le 01.04.2012. UCM. *

Secrétariat social UCM. (2017). Le régime de chômage avec complément d'entreprise. Wierde: UCM. *

Service fédéral des Pensions. (2017). Rapport annuel 2017. Bruxelles: Service fédéral des Pension.

Service fédéral des Pensions. (2019). Législation pensions salariés. Retrieved from Service fédéral des Pensions: http://www.onprvp.fgov.be *

Service fédéral des Pensions. (2019). Statistique annuelle des bénéficiaires de prestations . Bruxelles: Service fédéral des Pensions.

Service Public Fédéral Finances. (1989 - 2019). Mémento Fiscal 1989 - 2019. Bruxelles.

Service Public Fédéral Justice. (n.d.). Moniteur belge. Retrieved from Service Public Fédéral Justice: http://www.ejustice.just.fgov.be/cgi/welcome.pl *

* Sources for figures 1-4 


\section{APPENDIX}

\section{A.1 EVOLUTION OF LUMP-SUM WAGES}

\begin{tabular}{|c|cc|}
\hline $\begin{array}{c}\text { Every wage earner } \\
\text { before 1955 (except coal } \\
\text { miners) Annual amount }\end{array}$ & $\begin{array}{l}\text { White-collar workers from 1955 to 1957 } \\
\text { Daily amount for each day of work of }(>4 \\
\text { hours) }\end{array}$ \\
\hline \multirow{2}{*}{$15104.97 €$} & 1955 & $4.9517 €$ \\
& 1956 & $5.2747 €$ \\
& 1957 & $5.6376 €$ \\
\hline
\end{tabular}

Note: These amounts are expressed at the index 147.31 (1 $1^{\text {st }}$ of March 2020). Specific lump-sum wages exist for coal miners, flying staff, seasonal and workers who cross the borders to go to work. Details on these specific flat-rates wages can be found on the website of the National Public Pension Office (See 'Salaire forfaitaire, https://www.sfpd.fgov.be/fr/montant-de-lapension/calcul/types-de-pensions/salaries/salaires/salaireforfaitaire\#SalaireForfaitaireEmployesAvant1958) 


\section{A.2 HISTORICAL EVOLUTION OF THE ASSIMILATED WAGE FOR PERIODS SPENT RECEIVING A REPLACEMENT INCOME}

\begin{tabular}{ccc}
\hline Year & $\begin{array}{c}\text { Blue-collar } \\
\text { workers (daily } \\
\text { amount) }\end{array}$ & $\begin{array}{c}\text { White-collar } \\
\text { workers (daily } \\
\text { amount) }\end{array}$ \\
\hline 1955 & 3.7494 & 4.9517 \\
1956 & 3.9941 & 5.2747 \\
1957 & 4.2687 & 5.6376 \\
1598 & 4.4794 & 4.4794 \\
1959 & 4.6978 & 4.6978 \\
1960 & 4.8815 & 4.8815 \\
1961 & 5.1076 & 5.1076 \\
1962 & 5.3657 & 5.3657 \\
1963 & 5.6782 & 5.6782 \\
1964 & 6.1279 & 6.1279 \\
1965 & 6.6066 & 6.6066 \\
1966 & 7.1302 & 7.1302 \\
1967 & 7.5974 & 7.5974 \\
\hline
\end{tabular}

Note: These amounts are expressed at the index 147.31 (1 $1^{\text {st }}$ of March 2020). Specific lump-sum wages exist for assimilated periods of flying staff, coal miners, seasonal and cross border workers. (See 'Salaire forfaitaire,

https://www.sfpd.fgov.be/fr/montant-de-la-pension/calcul/types-de-pensions/salaries/salaires/salaireforfaitaire\#SalaireForfaitaireEmployesAvant1958) 
2.A.3 VALORISATION COEFFICIENTS FOR A PERSON RETIRING IN 2020 (PIVOTAL INDEX 147.31)

\begin{tabular}{|c|c|c|c|}
\hline Year & $\begin{array}{l}\text { Valorisation } \\
\text { coefficient }\end{array}$ & Year & $\begin{array}{l}\text { Valorisation } \\
\text { coefficient }\end{array}$ \\
\hline 1968 & 5.373152 & 1994 & 1.521279 \\
\hline 1969 & 5.179091 & 1995 & 1.497951 \\
\hline 1970 & 4.984738 & 1996 & 1.473572 \\
\hline 1971 & 4.777162 & 1997 & 1.454396 \\
\hline 1972 & 4.530205 & 1998 & 1.43612 \\
\hline 1973 & 4.235628 & 1999 & 1.422694 \\
\hline 1974 & 3.758936 & 2000 & 1.396436 \\
\hline 1975 & 3.333413 & 2001 & 1.359149 \\
\hline 1976 & 3.053588 & 2002 & 1.335346 \\
\hline 1977 & 2.851011 & 2003 & 1.31622 \\
\hline 1978 & 2.729096 & 2004 & 1.295135 \\
\hline 1979 & 2.612206 & 2005 & 1.267641 \\
\hline 1980 & 2.449358 & 2006 & 1.245603 \\
\hline 1981 & 2.275798 & 2007 & 1.223973 \\
\hline 1982 & 2.093128 & 2008 & 1.174374 \\
\hline 1983 & 1.944172 & 2009 & 1.167487 \\
\hline 1984 & 1.864744 & 2010 & 1.148294 \\
\hline 1985 & 1.813752 & 2011 & 1.114204 \\
\hline 1986 & 1.790545 & 2012 & 1.085444 \\
\hline 1987 & 1.798437 & 2013 & 1.072141 \\
\hline 1988 & 1.777797 & 2014 & 1.067858 \\
\hline 1989 & 1.724215 & 2015 & 1.078333 \\
\hline 1990 & 1.666742 & 2016 & 1.05623 \\
\hline 1991 & 1.614887 & 2017 & 1.037086 \\
\hline 1992 & 1.576587 & 2018 & 1.019101 \\
\hline 1993 & 1.534303 & 2019 & 1.004379 \\
\hline
\end{tabular}

Source: National Public Pension Office (see 'Revalorisation des salaires', https://www.sfpd.fgov.be/fr/montant-de-la-pension/calcul/types-depensions/salaries/salaires/revalorisation-des-salaires) 


\section{A.4 EVOLUTION OF THE PIVOTAL INDEX AND ADD-ON FACTOR}

\begin{tabular}{|c|c|c|c|c|c|}
\hline Date & $\begin{array}{l}\text { Pivotal } \\
\text { index }\end{array}$ & $\begin{array}{l}\text { Add-on } \\
\text { factor }\end{array}$ & Date & $\begin{array}{l}\text { Pivotal } \\
\text { index }^{134}\end{array}$ & $\begin{array}{l}\text { Add-on } \\
\text { factor }\end{array}$ \\
\hline 01.01 .1980 & 219.50 & 1.00 & 01.09 .2000 & 430.37 & 2.00 \\
\hline 01.03.1980 & 223.89 & 1.02 & 01.06.2001 & 438.98 & 2.04 \\
\hline 01.07.1980 & 228.37 & 1.04 & 01.01.2002 & $107.3^{*}$ & 2.08 \\
\hline 01.11 .1980 & 232.94 & 1.06 & 01.02 .2002 & $109.45^{*}$ & 2.12 \\
\hline 01.01.1981 & 237.60 & 1.08 & 01.06 .2003 & $111.64^{*}$ & 2.16 \\
\hline 01.04.1981 & 242.35 & 1.10 & 01.10 .2004 & $113.87^{*}$ & 2.21 \\
\hline 01.09.1981 & 247.20 & 1.13 & 01.08.2005 & $116.15^{*}$ & 2.25 \\
\hline 01.11.1981 & 252.14 & 1.15 & 01.10 .2006 & $118.47^{*}$ & 2.30 \\
\hline 01.02.1982 & 257.18 & 1.17 & 01.09.2007 & $118.47^{*}$ & 2.34 \\
\hline 01.05.1982 & 262.32 & 1.20 & 01.01.2008 & $120.84^{*}$ & 2.39 \\
\hline 01.08.1982 & 267.57 & 1.22 & 01.05 .2008 & $123.26^{*}$ & 2.44 \\
\hline 01.10 .1982 & 272.92 & 1.24 & 01.07.2008 & $123.26^{*}$ & 2.49 \\
\hline 01.12.1982 & 278.38 & 1.27 & 01.09.2008 & $125.73^{*}$ & 2.54 \\
\hline 01.04.1983 & 283.95 & 1.29 & 01.06.2009 & $125.73^{*}$ & 2.59 \\
\hline 01.09.1983 & 289.63 & 1.32 & 01.09 .2010 & $128.24^{*}$ & 2.64 \\
\hline 01.01.1984 & 295.42 & 1.35 & 01.05.2011 & $130.8^{*}$ & 2.69 \\
\hline 01.05.1984 & 301.33 & 1.37 & 01.02 .2012 & $133.42^{*}$ & 2.75 \\
\hline 01.08.1984 & 307.36 & 1.40 & 01.12 .2012 & $136.09^{*}$ & 2.80 \\
\hline 01.01.1985 & 313.51 & 1.43 & 01.06.2016 & $138.81^{*}$ & 2.86 \\
\hline 01.06.1985 & 319.78 & 1.46 & 01.06.2017 & $141.59^{*}$ & 2.91 \\
\hline 01.10 .1985 & 326.18 & 1.49 & & & \\
\hline 01.06.1987 & 332.70 & 1.52 & & & \\
\hline 01.11.1988 & 339.35 & 1.55 & & & \\
\hline 01.08.1989 & 346.14 & 1.58 & & & \\
\hline 01.02.1990 & 353.06 & 1.61 & & & \\
\hline 01.11.1990 & 360.12 & 1.64 & & & \\
\hline 01.03.1991 & 367.32 & 1.67 & & & \\
\hline 01.12.1991 & 374.67 & 1.71 & & & \\
\hline 01.11.1992 & 382.16 & 1.74 & & & \\
\hline 01.07.1993 & 389.80 & 1.78 & & & \\
\hline 01.12.1994 & 397.60 & 1.81 & & & \\
\hline 01.07.1995 & 397.60 & 1.85 & & & \\
\hline 01.05.1996 & 405.55 & 1.88 & & & \\
\hline 01.10.1997 & 413.66 & 1.92 & & & \\
\hline 01.06.1999 & 421.93 & 1.96 & & & \\
\hline
\end{tabular}

Source: National Public Pension Office and Service paiements traitements du secteur public ${ }^{135}$

$134 *$ Base 1996

${ }^{135}$ See 'Ajustement de

l'indice', https://www.traitements.fgov.be/indexation/default.htm\#L\%EF\%BF\%BDindice_pivot_\%EF\%BF\%BD_d\%EF\%BF\%BDpasser 


\section{A.5 HISTORICAL EVOLUTION OF GROWTH COEFFICIENTS}

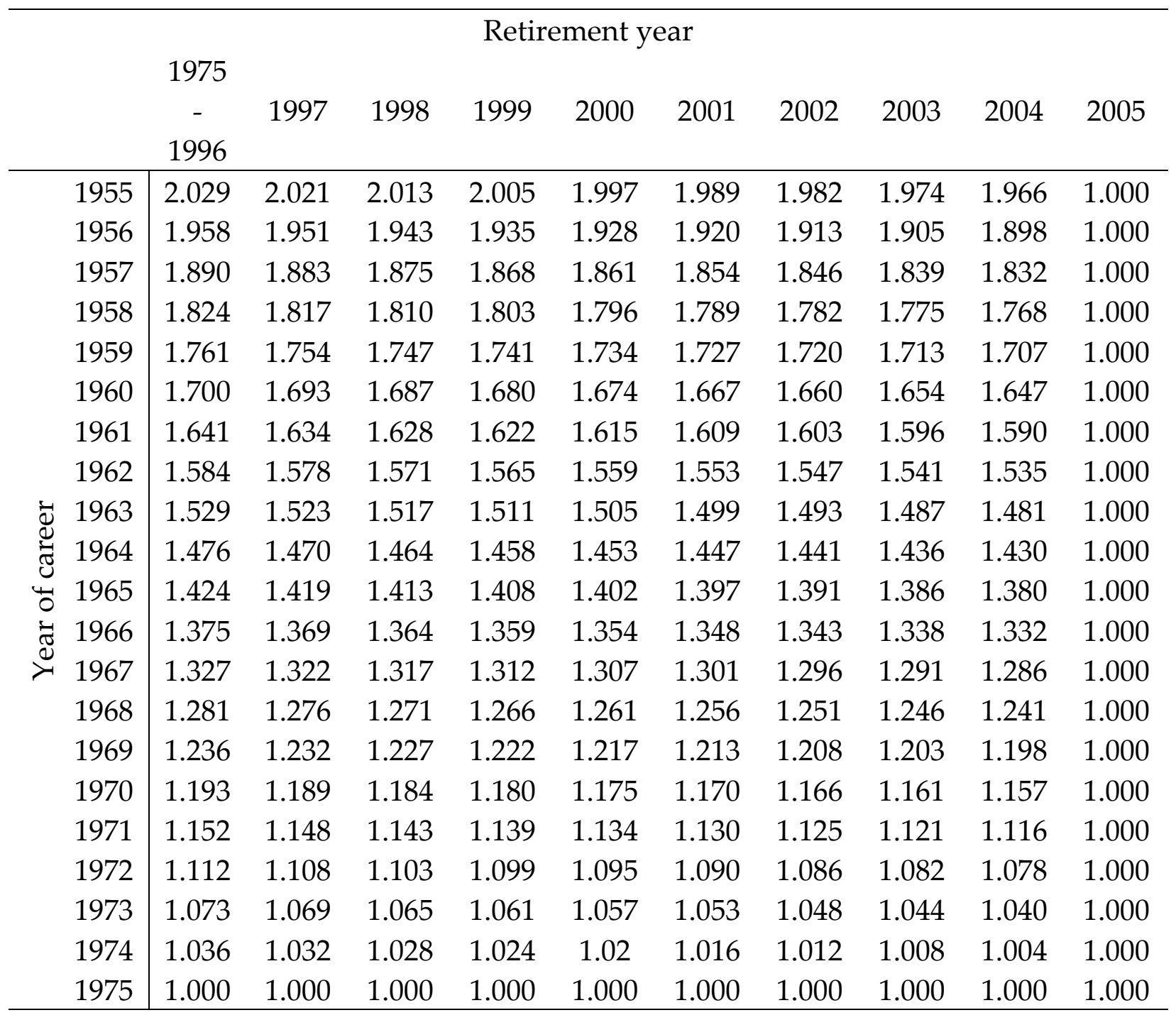

Source : Moniteur belge and Feestjens (1997) 


\section{A.6 EVOLUTION OF CEILINGS FOR PENSIONABLE EARNINGS}

\begin{tabular}{|c|c|c|c|c|}
\hline Year & $\begin{array}{l}\text { Wage } \\
\text { ceiling } \\
(\text { annual) })^{136}\end{array}$ & $+10 \%$ & Year & $\begin{array}{l}\text { Wage } \\
\text { ceiling } \\
\text { (annual) }\end{array}$ \\
\hline 1958 & $2379.76^{*}$ & $2617.76^{*}$ & 1991 & 30954.94 \\
\hline 1959 & $2379.76^{*}$ & $2617.76^{*}$ & 1992 & 31707.02 \\
\hline 1960 & $2469.01^{*}$ & $2715.92^{*}$ & 1993 & 32580.35 \\
\hline 1961 & $2498.76^{*}$ & $2748.64^{*}$ & 1994 & 32859.53 \\
\hline 1962 & $2528.52^{*}$ & $2781.36^{*}$ & 1995 & 33371.67 \\
\hline 1963 & $2558.28^{*}$ & $2814.08^{*}$ & 1996 & 33923.29 \\
\hline 1964 & $2651.22^{*}$ & $2916.34^{*}$ & 1997 & 34371.26 \\
\hline 1965 & $2777.66^{*}$ & $3055.42^{*}$ & 1998 & 34808.09 \\
\hline 1966 & $2885.49^{*}$ & $3174.04^{*}$ & 1999 & 36155.2 \\
\hline 1967 & $2963.58^{*}$ & $3259.92^{*}$ & 2000 & 36835.37 \\
\hline 1968 & $4008.44^{*}$ & $4409.28^{*}$ & 2001 & 38678.5 \\
\hline 1969 & $4714.93^{*}$ & $5186.42^{*}$ & 2002 & 39367.7 \\
\hline 1970 & $5071.91^{*}$ & $5579.09 *$ & 2003 & 40898.3 \\
\hline 1971 & $5270.82^{*}$ & $5797.93^{*}$ & 2004 & 41564.11 \\
\hline 1972 & $5572.03^{*}$ & $6129.27^{*}$ & 2005 & 43314.93 \\
\hline 1973 & $7793.77^{*}$ & & 2006 & 44081.27 \\
\hline 1974 & $9645.53^{*}$ & & 2007 & 44994.88 \\
\hline 1975 & $11623.72^{*}$ & & 2008 & 46895.18 \\
\hline 1976 & $13347.2^{*}$ & & 2009 & 47171.84 \\
\hline 1977 & $14300.96^{*}$ & & 2010 & 47960.29 \\
\hline 1978 & $14955.41^{*}$ & & 2011 & 49773.66 \\
\hline 1979 & $15632.16^{*}$ & & 2012 & 51092.44 \\
\hline 1980 & 16595.23 & & 2013 & 52760.95 \\
\hline 1981 & 20697.62 & & 2014 & 52972.54 \\
\hline 1982 & 22503.5 & & 2015 & 53528.57 \\
\hline 1983 & 24228.12 & & 2016 & 54648.7 \\
\hline 1984 & 25765.9 & & 2017 & 55657.47 \\
\hline 1985 & 27020.1 & & 2018 & 57602.62 \\
\hline 1986 & 27370.25 & & 2019 & 58446.94 \\
\hline 1987 & 27795.38 & & & \\
\hline 1988 & 28118.69 & & & \\
\hline 1989 & 28992.19 & & & \\
\hline 1990 & 29991.7 & & & \\
\hline
\end{tabular}

Source: National Public Pension Office ${ }^{137}$

136 * Only applicable to white-collar workers

${ }_{137}$ See 'Plafond salarial', https://www.sfpd.fgov.be/fr/montant-de-la-pension/calcul/types-depensions/salaries/salaires/plafond-salarial 


\section{A.7 DIFFERENTIATED CEILINGS FOR ASSIMILATED PERIODS FOR WAGE- EARNERS}

\begin{tabular}{cc}
\hline Year & $\begin{array}{c}\text { Differentiated ceiling } \\
\text { for assimilated periods }\end{array}$ \\
\hline 2007 & 44860.29 \\
2008 & 46754.91 \\
2009 & 47030.73 \\
2010 & 47816.83 \\
2011 & 49279.82 \\
2012 & 50585.52 \\
2013 & 51213.21 \\
2014 & 51418.58 \\
2015 & 51958.31 \\
2016 & 53045.58 \\
2017 & 54024.76 \\
2018 & 54978.21 \\
2019 & 55784.07 \\
\hline
\end{tabular}

Source: National Pension Office website ${ }^{137}$ 


\section{A.8 EARNINGS TEST FOR THE COMBINATION OF WORK AND OLD-AGE PENSION RECEIPT, 2020 (PIVOTAL INDEX 147.31)}

\begin{tabular}{|c|c|c|c|}
\hline \multirow{2}{*}{ Eligibility conditions } & \multirow{2}{*}{ Family allowances } & \multicolumn{2}{|c|}{$\begin{array}{l}\text { Earnings threshold by type of activity } \\
\text { (annual) }\end{array}$} \\
\hline & & $\begin{array}{l}\text { Wage-earner or } \\
\text { civil servant }\end{array}$ & $\begin{array}{l}\text { Self-employed } \\
\text { worker }\end{array}$ \\
\hline $\begin{array}{l}\text { Aged more than } 65 \\
\text { (with or without a } \\
\text { survivor pension) }\end{array}$ & N.A & No earnings test & No earnings test \\
\hline $\begin{array}{c}\text { Career of more than } 45 \\
\text { years }\end{array}$ & N.A & No earnings test & No earnings test \\
\hline Aged less than 65 and & No & 8,393 & 6,714 \\
\hline $\begin{array}{c}\text { career of less than } 45 \\
\text { years } \\
\text { OR } \\
\text { Aged less than } 65 \text { and } \\
\text { the spouse receives an } \\
\text { old-age pension benefit } \\
\text { at the household } \\
\text { replacement rate }\end{array}$ & Yes & 12,590 & 10,071 \\
\hline \multirow{2}{*}{$\begin{array}{l}\text { Aged less than } 65 \text { and } \\
\text { receiving a survivor } \\
\text { pension only }\end{array}$} & No & 19,542 & 15,634 \\
\hline & Yes & 24,428 & 19,542 \\
\hline $\begin{array}{l}\text { Aged more than } 65 \text { and } \\
\text { a spouse that receives } \\
\text { the old-age pension } \\
\text { ebenfit at the household } \\
\text { replacement rate } \\
\text { Or } \\
\text { Aged more than } 65 \text { and } \\
\text { receiving a survivor } \\
\text { pension only }\end{array}$ & N.A & 29,489 & 23,591 \\
\hline
\end{tabular}

Source: National pension office website (See 'Puis-je encore travailler si je prends ma pension ou si mon conjoint bénéficie d'une pension au taux ménage ?', https://www.sfpd.fgov.be/fr/montant-de-lapension/travailler\#: :text=Attention\%20\%3A\%20si\%20votre\%20pension\%20de,normalement\%20pour\%20 une $\% 20$ ann $\%$ C3\%A9e\%20compl\%C3\%A8te.) 


\section{A.9 CEILINGS FOR SICKNESS AND DISABILITY BENEFITS}

\begin{tabular}{|c|c|c|c|c|c|c|c|c|c|}
\hline \multirow[b]{2}{*}{$\begin{array}{c}\text { Year of } \\
\text { benefits receipt } \\
\text { (last pivotal } \\
\text { index at the } \\
\text { end of the civil } \\
\text { year) }\end{array}$} & \multicolumn{9}{|c|}{ Start of the sickness or disability benefits } \\
\hline & $\begin{array}{c}< \\
1^{\text {st }} \\
\text { Jan. } \\
2005\end{array}$ & $\begin{array}{c}1^{\text {st Jan. }} \\
2005 \\
- \\
31^{\text {st }} \\
\text { Dec. } \\
2006\end{array}$ & $\begin{array}{c}1^{\text {st }} \text { Jan. } \\
2007 \\
- \\
31^{\text {st }} \\
\text { Dec. } \\
2008\end{array}$ & $\begin{array}{c}1^{\text {st }} \text { Jan. } \\
2009 \\
- \\
31^{\text {st }} \\
\text { Dec. } \\
2010\end{array}$ & $\begin{array}{l}1^{\text {st Jan. }} \\
2011 \\
- \\
31^{\text {st }} \\
\text { Mar. } \\
2013\end{array}$ & $\begin{array}{c}1^{\text {st }} \\
\text { Apr. } \\
2013 \\
- \\
31^{\text {st }} \\
\text { Marc. } \\
2015 \\
\end{array}$ & $\begin{array}{c}1^{\text {st }} \\
\text { Apr. } \\
2015 \\
- \\
31^{\text {st }} \\
\text { Dec. } \\
2017 \\
\end{array}$ & $\begin{array}{c}1^{\text {st }} \text { Jan. } \\
2018 \\
- \\
31 \text { st } \\
\text { Dec. } \\
2019\end{array}$ & $\begin{array}{c}> \\
1^{\text {st }} \text { Jan. } \\
2020\end{array}$ \\
\hline 2000 & 95.37 & 97.28 & 98.25 & 99.04 & 99.73 & 101.72 & 103.00 & 103.82 & 104.96 \\
\hline 2001 & 97.28 & 99.22 & 100.22 & 101.02 & 101.72 & 103.76 & 105.06 & 105.90 & 107.06 \\
\hline 2002 & 99.22 & 101.21 & 102.22 & 103.04 & 103.76 & 105.83 & 107.16 & 108.01 & 109.20 \\
\hline 2003 & 101.21 & 103.23 & 104.27 & 105.10 & 105.84 & 107.95 & 109.30 & 110.18 & 111.39 \\
\hline 2004 & 103.23 & 105.30 & 106.35 & 107.20 & 107.95 & 110.11 & 111.49 & 112.38 & 113.61 \\
\hline 2005 & 105.30 & 107.40 & 108.48 & 109.35 & 110.11 & 112.31 & 113.72 & 114.63 & 115.89 \\
\hline 2006 & 107.40 & 109.55 & 110.64 & 111.53 & 112.31 & 114.56 & 115.99 & 116.92 & 118.20 \\
\hline 2007 & 107.40 & 109.55 & 110.64 & 111.53 & 112.31 & 114.56 & 115.99 & 116.92 & 118.20 \\
\hline 2008 & 113.98 & 116.26 & 117.43 & 118.36 & 119.19 & 121.58 & 123.10 & 124.08 & 125.45 \\
\hline 2009 & 113.98 & 116.26 & 117.43 & 118.36 & 119.19 & 121.58 & 123.10 & 124.08 & 125.45 \\
\hline 2010 & 116.26 & 118.58 & 119.77 & 120.73 & 121.57 & 124.00 & 125.55 & 126.56 & 127.95 \\
\hline 2011 & 118.58 & 120.95 & 122.16 & 123.14 & 124.00 & 126.48 & 128.06 & 129.08 & 130.50 \\
\hline 2012 & 123.37 & 125.84 & 127.10 & 128.12 & 129.01 & 131.59 & 133.24 & 134.31 & 135.78 \\
\hline 2013 & 123.37 & 125.84 & 127.10 & 128.12 & 129.01 & 131.59 & 133.24 & 134.31 & 135.78 \\
\hline 2014 & 123.37 & 125.84 & 127.10 & 128.12 & 129.01 & 131.59 & 133.24 & 134.31 & 135.78 \\
\hline 2015 & 123.37 & 125.84 & 127.10 & 128.12 & 129.01 & 131.59 & 133.24 & 134.31 & 135.78 \\
\hline 2016 & 125.84 & 128.36 & 129.64 & 130.68 & 131.59 & 134.22 & 135.90 & 136.99 & 138.50 \\
\hline 2017 & 128.36 & 130.93 & 132.24 & 133.30 & 134.23 & 136.91 & 138.62 & 139.73 & 141.27 \\
\hline 2018 & 130.93 & 133.55 & 134.88 & 135.96 & 136.91 & 139.65 & 141.40 & 142.53 & 144.10 \\
\hline 2019 & 130.93 & 133.55 & 134.88 & 135.96 & 136.91 & 139.65 & 141.40 & 142.53 & 144.10 \\
\hline 2020 & 133.55 & 136.22 & 137.58 & 138.68 & 139.65 & 142.44 & 144.22 & 145.38 & 146.98 \\
\hline
\end{tabular}

Source: National Sickness and disability benefits Institute website ${ }^{138}$

138 See 'Votre plafond salarial', https://www.inami.fgov.be/fr/themes/incapacite-travail/montants/salarieschomeurs/Pages/indemnite-plafond-salarial-salarie.aspx 


\section{A.10 TIME CREDIT BENEFITS (MONTHLY NET AMOUNTS)}

\begin{tabular}{|c|c|c|c|c|c|}
\hline & \multicolumn{2}{|c|}{$1 / 2$ work interruption } & \multicolumn{3}{|c|}{$1 / 5$ work interruption } \\
\hline & \multirow[b]{2}{*}{$\begin{array}{c}\text { Legal } \\
\text { cohabitants }\end{array}$} & \multirow[b]{2}{*}{ Isolated } & \multirow[b]{2}{*}{$\begin{array}{c}\text { Legal } \\
\text { Cohabitant }\end{array}$} & \multicolumn{2}{|c|}{ Isolated } \\
\hline & & & & $\begin{array}{c}\text { Without } \\
\text { dependent } \\
\text { children }\end{array}$ & $\begin{array}{c}\text { With } \\
\text { dependent } \\
\text { children }\end{array}$ \\
\hline 2002 & 250.42 & 319.19 & 116.32 & 140.37 & 178.92 \\
\hline 2003 & 255.43 & 325.58 & 118.65 & 143.18 & 182.50 \\
\hline 2004 & 260.53 & 332.08 & 121.02 & 146.04 & 186.14 \\
\hline 2005 & 265.75 & 338.73 & 123.44 & 148.97 & 189.87 \\
\hline 2006 & 271.05 & 345.49 & 125.91 & 151.94 & 193.66 \\
\hline 2007 & 271.05 & 345.49 & 125.91 & 151.94 & 193.66 \\
\hline 2008 & 287.67 & 366.67 & 133.62 & 161.25 & 205.53 \\
\hline 2009 & 287.67 & 366.67 & 133.62 & 161.25 & 205.53 \\
\hline 2010 & 293.41 & 373.99 & 136.29 & 164.47 & 209.64 \\
\hline 2011 & 299.27 & 381.45 & 139.01 & 167.76 & 213.82 \\
\hline 2012 & 311.37 & 396.88 & 144.64 & 174.54 & 222.47 \\
\hline 2013 & 311.37 & 396.88 & 144.64 & 174.54 & 222.47 \\
\hline 2014 & 311.37 & 396.88 & 144.64 & 174.54 & 222.47 \\
\hline 2015 & 311.37 & 396.88 & 144.64 & 174.54 & 222.47 \\
\hline 2016 & 317.59 & 404.81 & 147.53 & 178.03 & 226.91 \\
\hline 2017 & 323.95 & 412.92 & 150.48 & 181.59 & 231.46 \\
\hline 2018 & 330.43 & 421.18 & 153.49 & 185.23 & 236.09 \\
\hline 2019 & 337.04 & 429.60 & 156.56 & 188.93 & 240.81 \\
\hline 2020 & 337.04 & 429.6 & 156.56 & 188.93 & 240.81 \\
\hline
\end{tabular}

Source: National employment Office website (' Documentation - Interruption de carrière - Crédit -temps', https://www.onem.be/fr/documentation/montants-baremes/interruption-de-carriere-credit-temps\#16138) 


\section{A.11 HISTORICAL EVOLUTION OF THE FIRST TAX DEDUCTION FOR}

REPLACEMENT INCOME

\begin{tabular}{|c|c|c|c|c|c|c|c|c|}
\hline \multirow[b]{2}{*}{$\begin{array}{l}\text { Year of } \\
\text { income }\end{array}$} & \multicolumn{2}{|c|}{ Old-age pension } & \multicolumn{2}{|c|}{$\begin{array}{c}\text { Conventional early } \\
\text { retirement }\end{array}$} & \multicolumn{2}{|c|}{$\begin{array}{c}\text { Old-age } \\
\text { unemployment }\end{array}$} & \multicolumn{2}{|c|}{ Disability } \\
\hline & Isolated & $\begin{array}{l}\text { Single } \\
\text { earner }\end{array}$ & Isolated & $\begin{array}{l}\text { Single } \\
\text { earner }\end{array}$ & Isolated & $\begin{array}{l}\text { Single } \\
\text { earner }\end{array}$ & Isolated & $\begin{array}{l}\text { Single } \\
\text { earner }\end{array}$ \\
\hline 1986 & 1551.6 & 1551.6 & 2488.5 & 2478.7 & 1551.6 & 1551.6 & 1561.5 & 1561.5 \\
\hline 1987 & 1574.7 & 1574.7 & 2521.6 & 2478.7 & 1574.7 & 1574.7 & 1930.1 & 1930.1 \\
\hline 1988 & 1530.0 & 1530.0 & 2478.7 & 2478.7 & 1530.0 & 1530.0 & 1883.3 & 1883.3 \\
\hline 1989 & 1271.7 & 1488.6 & 2243.3 & 2460.2 & 1271.7 & 1488.6 & 1642.3 & 1859.2 \\
\hline 1990 & 1271.7 & 1488.6 & 2243.3 & 2460.2 & 1271.7 & 1488.6 & 1642.3 & 1859.2 \\
\hline 1991 & 1381.9 & 1611.2 & 2346.1 & 2696.9 & 1381.9 & 1611.2 & 1779.8 & 2009.1 \\
\hline 1992 & 1420.5 & 1643.6 & 2568.5 & 2804.0 & 1420.5 & 1643.6 & 1830.1 & 2065.6 \\
\hline 1993 & 1478.8 & 1726.7 & 2677.6 & 2925.5 & 1478.8 & 1726.7 & 1898.2 & 2146.1 \\
\hline 1994 & 1478.8 & 1726.7 & 2677.6 & 2925.5 & 1478.8 & 1726.7 & 1898.2 & 2146.1 \\
\hline 1995 & 1478.8 & 1726.7 & 2677.6 & 2925.5 & 1478.8 & 1726.7 & 1898.2 & 2146.1 \\
\hline 1996 & 1478.8 & 1726.7 & 2677.6 & 2925.5 & 1478.8 & 1726.7 & 1898.2 & 2146.1 \\
\hline 1997 & 1478.8 & 1726.7 & 2677.6 & 2925.5 & 1478.8 & 1726.7 & 1898.2 & 2146.1 \\
\hline 1998 & 1478.8 & 1726.7 & 2677.6 & 2925.5 & 1478.8 & 1726.7 & 1898.2 & 2146.1 \\
\hline 1999 & 1492.9 & 1743.1 & 1492.9 & 1743.1 & 1492.9 & 1743.1 & 1916.4 & 2166.6 \\
\hline 2000 & 1509.6 & 1762.8 & 1509.6 & 1762.6 & 1509.6 & 1762.6 & 1937.8 & 2190.8 \\
\hline 2001 & 1550.0 & 1810.0 & 1550.0 & 1810.0 & 1550.0 & 1810.0 & 1990.0 & 2250.0 \\
\hline 2002 & 1590.0 & 1850.0 & 1590.0 & 1850.0 & 1590.0 & 1850.0 & 2040.0 & 2300.0 \\
\hline 2003 & 1612.3 & 1882.5 & 1612.3 & 1882.5 & 1612.3 & 1882.5 & 2069.6 & 2339.9 \\
\hline 2004 & 1638.0 & 1638.0 & 1638.0 & 1912.5 & 1638.0 & 1912.5 & 2102.6 & 2102.6 \\
\hline 2005 & 1672.2 & 1672.2 & 1672.2 & 1672.2 & 1672.2 & 1952.3 & 2146.6 & 2146.6 \\
\hline 2006 & 1718.9 & 1718.9 & 1718.9 & 1718.9 & 1718.9 & 2007.0 & 2206.5 & 2206.5 \\
\hline 2007 & 1748.7 & 1748.7 & 1748.7 & 1748.7 & 1748.7 & 2042.6 & 2242.0 & 2242.0 \\
\hline 2008 & 1781.3 & 1781.3 & 1781.3 & 1781.3 & 1781.3 & 2079.9 & 2286.6 & 2286.6 \\
\hline 2009 & 1861.4 & 1861.4 & 1861.4 & 1861.4 & 1861.4 & 1861.4 & 2389.5 & 2389.5 \\
\hline 2010 & 1861.4 & 1861.4 & 1861.4 & 1861.4 & 1861.4 & 1861.4 & 2389.5 & 2389.5 \\
\hline 2011 & 1901.1 & 1901.1 & 1901.1 & 1901.1 & 1901.1 & 1901.1 & 2440.4 & 2440.4 \\
\hline 2012 & 1968.2 & 1968.2 & 1968.2 & 1968.2 & 1968.2 & 1968.2 & 2526.5 & 2526.5 \\
\hline 2013 & 2024.1 & 2024.1 & 2024.1 & 2024.1 & 2024.1 & 2024.1 & 2598.3 & 2598.3 \\
\hline 2014 & 2024.1 & 2024.1 & 2024.1 & 2024.1 & 2024.1 & 2024.1 & 2598.3 & 2598.3 \\
\hline 2015 & 2024.1 & 2024.1 & 2024.1 & 2024.1 & 2024.1 & 2024.1 & 2598.3 & 2598.3 \\
\hline 2016 & 2024.1 & 2024.1 & 2024.1 & 2024.1 & 2024.1 & 2024.1 & 2598.3 & 2598.3 \\
\hline 2017 & 2024.1 & 2024.1 & 2024.1 & 2024.1 & 2024.1 & 2024.1 & 2598.3 & 2598.3 \\
\hline 2018 & 2024.1 & 2024.1 & 2024.1 & 2024.1 & 2024.1 & 2024.1 & 2598.3 & 2598.3 \\
\hline 2019 & 2066.9 & 2066.9 & 2066.9 & 2066.9 & 2066.9 & 2066.9 & 2653.2 & 2653.2 \\
\hline
\end{tabular}

Source: Service Public Fédéral - Mémento Fiscal (1989 - 2019) 


\section{A.12 HISTORICAL EVOLUTION OF THE SECOND TAX DEDUCTION FOR}

REPLACEMENT INCOME

\begin{tabular}{|c|c|c|c|c|}
\hline $\begin{array}{l}\text { Year of } \\
\text { income }\end{array}$ & $\begin{array}{l}\text { Old-age } \\
\text { pension }\end{array}$ & $\begin{array}{c}\text { Conventional } \\
\text { early retirement }\end{array}$ & $\begin{array}{c}\text { Old-age } \\
\text { unemployment }\end{array}$ & Disability \\
\hline 1979 & 8373.6 & 8373.6 & 8373.6 & 9304.0 \\
\hline 1980 & 8373.6 & 8373.6 & 8373.6 & 9304.0 \\
\hline 1981 & 8373.6 & 8373.6 & 8373.6 & 9304.0 \\
\hline 1982 & 8373.6 & 8373.6 & 8373.6 & 9304.0 \\
\hline 1983 & 8373.6 & 8373.6 & 8373.6 & 9304.0 \\
\hline 1984 & 8373.6 & 8373.6 & 8373.6 & 9304.0 \\
\hline 1985 & 8373.6 & 8373.6 & 8373.6 & 9304.0 \\
\hline 1986 & 8373.6 & 8373.6 & 8373.6 & 9304.0 \\
\hline 1987 & 8373.6 & 8373.6 & 8373.6 & 9304.0 \\
\hline 1988 & 8586.3 & 8586.3 & 8586.3 & 9264.9 \\
\hline 1989 & 8871.2 & 8871.2 & 9794.2 & 9856.9 \\
\hline 1990 & 9213.9 & 9213.9 & 10189.0 & 10252.7 \\
\hline 1991 & 9091.7 & 9091.7 & 10479.5 & 10542.6 \\
\hline 1992 & 9711.4 & 9711.4 & 10724.1 & 10790.5 \\
\hline 1993 & 9824.2 & 9824.2 & 10850.4 & 10915.8 \\
\hline 1994 & 9969.5 & 9969.5 & 10997.5 & 11077.2 \\
\hline 1995 & 10168.7 & 10168.7 & 11230.0 & 11298.6 \\
\hline 1996 & 10256.0 & 10256.0 & 11324.9 & 11395.6 \\
\hline 1997 & 10412.7 & 10412.7 & 11498.9 & 11569.6 \\
\hline 1998 & 10531.3 & 10531.3 & 11631.2 & 11701.4 \\
\hline 1999 & 10686.6 & 10686.6 & 11803.9 & 11874.0 \\
\hline 2000 & 10958.7 & 10958.7 & 12105.1 & 12176.3 \\
\hline 2001 & 11849.0 & 11849.0 & 13023.7 & 13165.3 \\
\hline 2002 & 12008.0 & 12008.0 & 13197.7 & 13342.3 \\
\hline 2003 & 12206.4 & 12206.4 & 13415.3 & 13562.6 \\
\hline 2004 & 12452.1 & 12452.1 & 13686.5 & 13835.7 \\
\hline 2005 & 12618.1 & 12618.1 & 13819.2 & 14020.1 \\
\hline 2006 & 12797.0 & 12797.0 & 14250.9 & 14418.9 \\
\hline 2007 & 13546.8 & 13546.8 & 14876.2 & 15052.0 \\
\hline 2008 & 13881.6 & 13881.6 & 15391.3 & 15423.9 \\
\hline 2009 & 13974.0 & 13974.0 & 15491.0 & 15526.6 \\
\hline 2010 & 14498.0 & 14498.0 & 16072.0 & 16108.9 \\
\hline 2011 & 14914.7 & 14914.7 & 16538.9 & 16571.9 \\
\hline 2012 & 15443.1 & 15443.1 & 17477.1 & 17159.0 \\
\hline 2013 & 15518.5 & 15518.5 & 17569.5 & 17242.8 \\
\hline 2014 & 15518.5 & 15518.5 & 17569.5 & 17242.8 \\
\hline 2015 & 15518.5 & 15518.5 & 17569.5 & 17242.8 \\
\hline 2016 & 15568.1 & 15568.1 & 17631.1 & 17297.9 \\
\hline 2017 & 15568.1 & 15568.1 & 17631.1 & 17297.9 \\
\hline 2018 & 15615.9 & 15615.9 & 17681.7 & 17351.0 \\
\hline
\end{tabular}

Source: Service Public Fédéral - Mémento Fiscal (1989 - 2019) 


\section{FINANCIAL RETIREMENT INCENTIVES IN BELGIUM: AN ANALYSIS OF FOUR DECADES OF CHANGE}

Anne-Lore Fraikin, Alain Jousten, Mathieu Lefebvre 


\subsection{INTRODUCTION}

Previous studies have highlighted the decisive role of public social security schemes in explaining the retirement patterns and low labor force participation of the elderly in Belgium (see among others Pestieau and Stijns, 1999; Dellis et al, 2004 or Jousten and Lefebvre, 2013). A common feature of these studies has been that they relied on a crosssectional, micro-based and supply side approach. Relying on detailed modeling of individual retirement incentives in a given reference period, the papers documented the presence of strong retirement incentives stemming from the social security system and their importance in explaining observed labor supply and retirement patterns.

The cross-sectional design of the research question of these papers however limits their ability to explain observed labor supply and retirement patterns of Figure 3.1. Since the start of standardized collection of labor force data by the Labor Force Survey (LFS) in 1984, cohorts of older women have seen a steady upward trend in their employment rates; male employment has gone through a U-shaped pattern, with a first period of decreased employment (up to the early 1980's) being followed by a period of stagnation (late 1980's to late 1990 's) before finally contributing to the previously mentioned significant increase in employment rates of older cohorts since the early 2000's. 
Figure 3.1: Employment rate of older workers (men and women, ages 55-64)
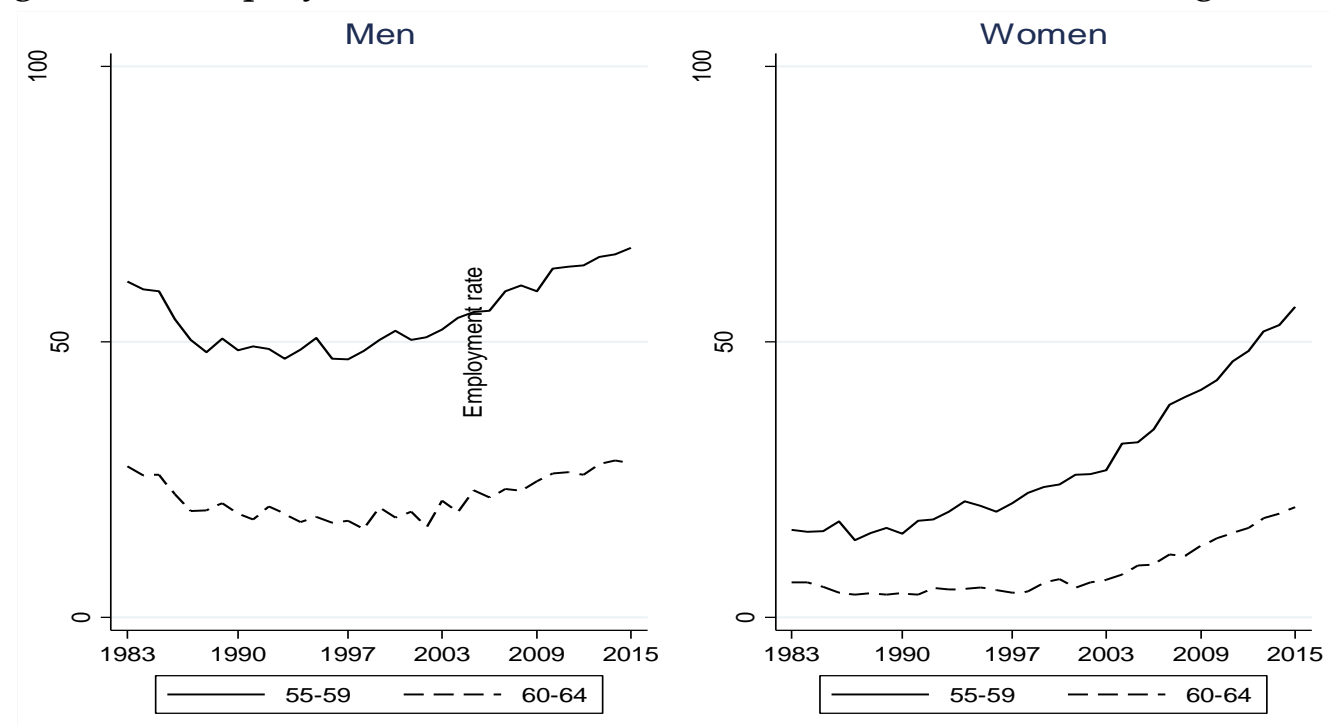

Source : Eurostat Labor Force Survey (2017)

The respective importance of individual incentives and institutional changes in explaining observed labor supply and retirement patterns over the last decades remains unclear. Expressed differently, the decomposition into age and year effects remains an important and so far understudied research question in the Belgian context. Jousten and Lefebvre (2016) delivered a first step towards filling this gap in the literature. First, they provided a summary of institutional changes over the last decades - with a focus not only on pension and early retirement schemes, but also unemployment, disability and time credit. Second, they provided a detailed macro-analysis of observed employment and retirement patterns, looking not only at trends in employment and labor force participation rates, but also at the changing intensity (e.g., more part-time work) and sectoral composition (e.g., a shift to service sectors) of employment in light of institutional changes.

This chapter goes further as we systematically calculate the financial incentives to exit employment for typical workers, by age, year and sex - integrating changes both in benefits and in the tax system. More specifically, we construct a simulation model aimed 
at assessing the incentives to retire for different subgroups of the population and link these incentives with their labor outcomes. The model allows us to separate age and year effects, incidentally also permitting us to take phased reforms and grandfathering provisions into account. The model also permits the study of an array of scenarios in terms of earnings level, earnings growth, mortality, as well as a simulation of modified system parameters.

We focus our attention on the population of wage-earners, leaving aside self-employed and civil servants. We further focus our attention on single workers, hence staying clear of issues of joint or spousal retirement decisions ${ }^{139}$ as well as interactions between individuals participating in different schemes. The reasons for these restrictions are linked to the overall complexity of social insurance and retirement programs in Belgium combined with a lack of structured historical information on some of them. The wage earner scheme is by far the most important scheme in terms of enrolments and scope of coverage - encompassing all private sector workers and contractual workers in the public sector. Furthermore, it is the scheme with the most time-series information available on the applicable institutional setting and on the characteristics of participants. It is also the scheme that has been most extensively studied so far (see Jousten et al, 2012; 2016 and Jousten and Lefebvre; 2016 and 2017). ${ }^{140}$

The structure of the chapter is as follows. Section 3.2 documents the changes in observed retirement patterns in Belgium. Section 2.3 introduces the retirement incentive indicator, with a focus on singles. Section 3.4 explores the link between observed retirement patterns

\footnotetext{
${ }^{139}$ See Jousten and Lefebvre (2017) and chapter six for a study of the role of spousal retirement incentives in the Belgian case.

140 The civil servant scheme displays a large heterogeneity, with only limited historical information available on institutional details as well as participants. The self-employed scheme is the least well documented, as the (substantially more limited) information on participants' earnings histories has only recently been the subject of centralization efforts.
} 
and incentives using a common standardized earnings profile combined with standardized tax parameters across time and countries. The common earnings profile and time-invariant tax parameters are chosen to allow for an easy international comparison of results. Section 3.5 deviates from this common profile by introducing specificities that are more relevant for understanding the Belgian case by using different and more realistic assumptions in terms of earnings levels, career profiles and taxation. It also discusses how benefit floors and ceilings affect incentives given these earnings histories. Section 3.6 concludes.

\subsection{PROGRAM PARTICIPATION}

In this section, we present the evolution of the pathways to retirement over the period 1983 to 2015. Figures 3.2 to 3.5 show for each year, and by age group, the percentage of men and women recorded as beneficiaries of each program. On each figure, we see how the structure changed over time with the implementation of reforms in some programs as well as the introduction of new programs.

Over our observation period, some pathways have gained or lost importance in the distribution of exits, reflecting the varying generosity in eligibility conditions. These figures document the connected vessels aspect of the various social security schemes. For men aged 55-59 we observe an increase in the proportion of recipients of unemployment and disability benefits since 2005, at a time when conventional early retirement rules were tightened. For men aged 60-64, the proportion of old-age pensioners has increased since 2005. For women aged 55-59, the picture is similar than for men except for the fact that we observe an increase in the participation in pathways other than the old-age pension since the end of the nineties. Such increase is due to the increase of the old-age pension statutory eligibility age, hence delaying the mechanical rollover of disability, 
unemployment conventional early retirement beneficiaries into the old-age pension program.

Figure 3.2: Pathways to retirement - men, age 55-59

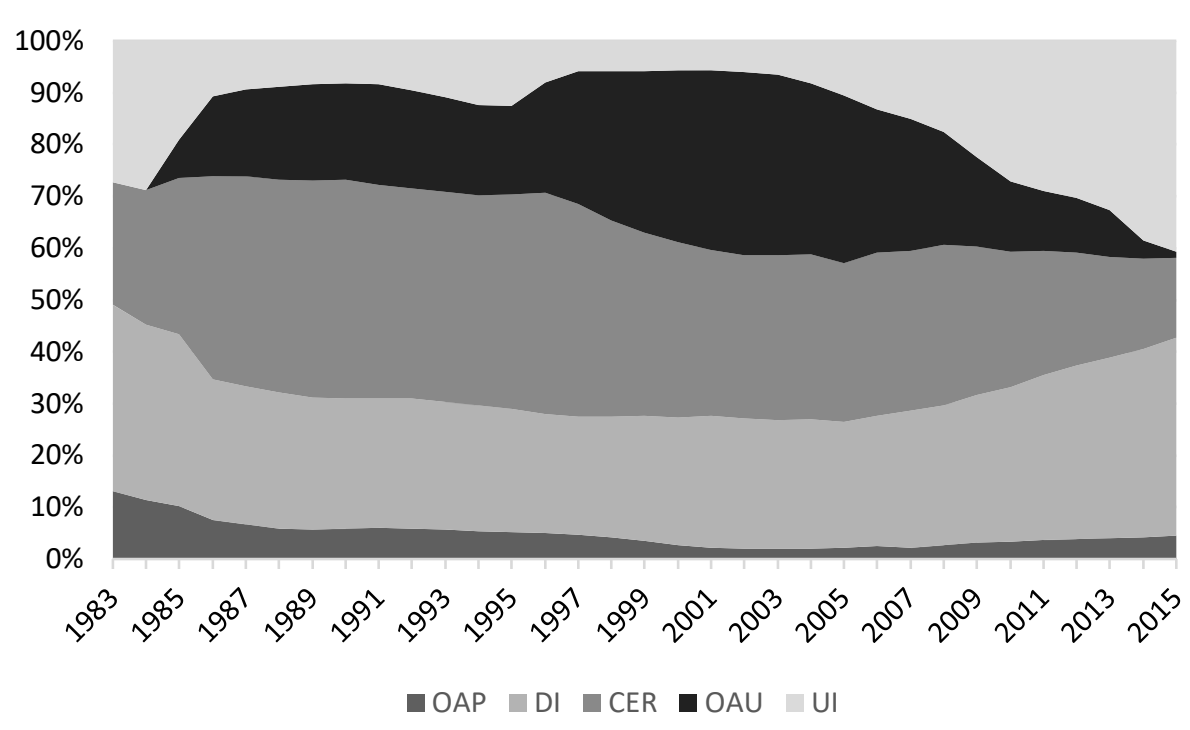

Source: National Pension Office (2019), Office National de l'Emploi (2021), National Institute of Incapacity and Invalidity (2020)

Figure 3.3: Pathways to retirement - men, age 60-64

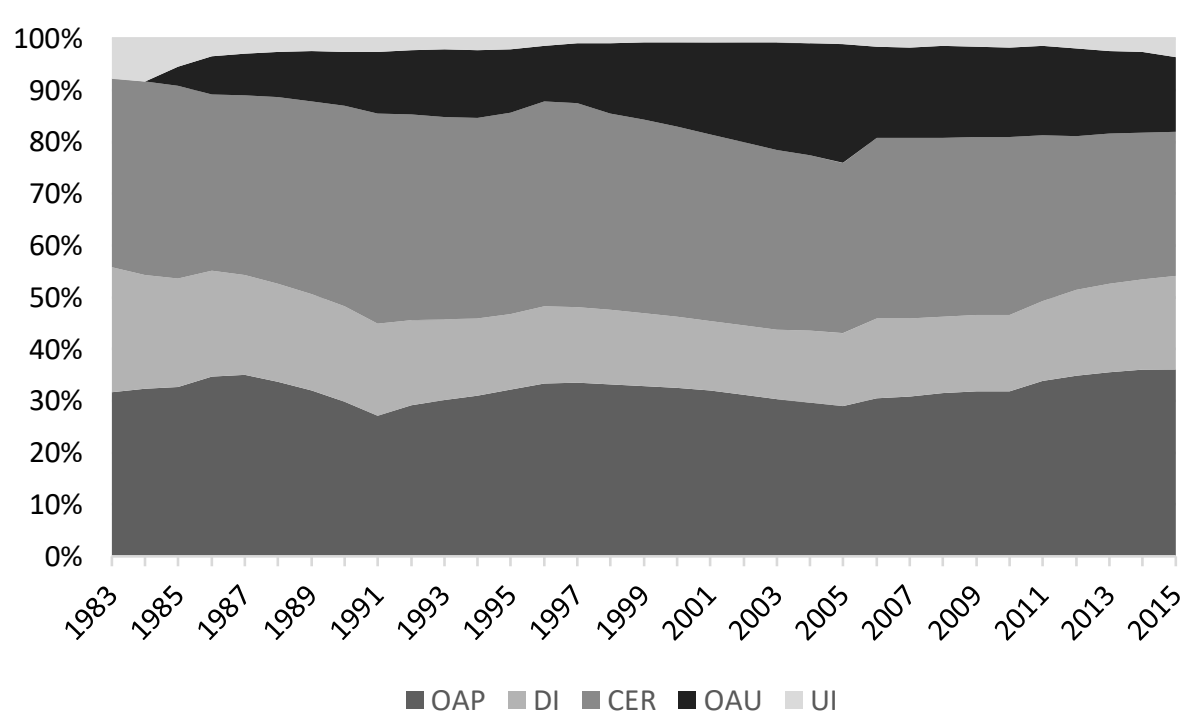

Source: National Pension Office (2019), Office National de l'Emploi (2021), National Institute of Incapacity and Invalidity (2020) 
Figure 3.4: Pathways to retirement - women, age 55-59

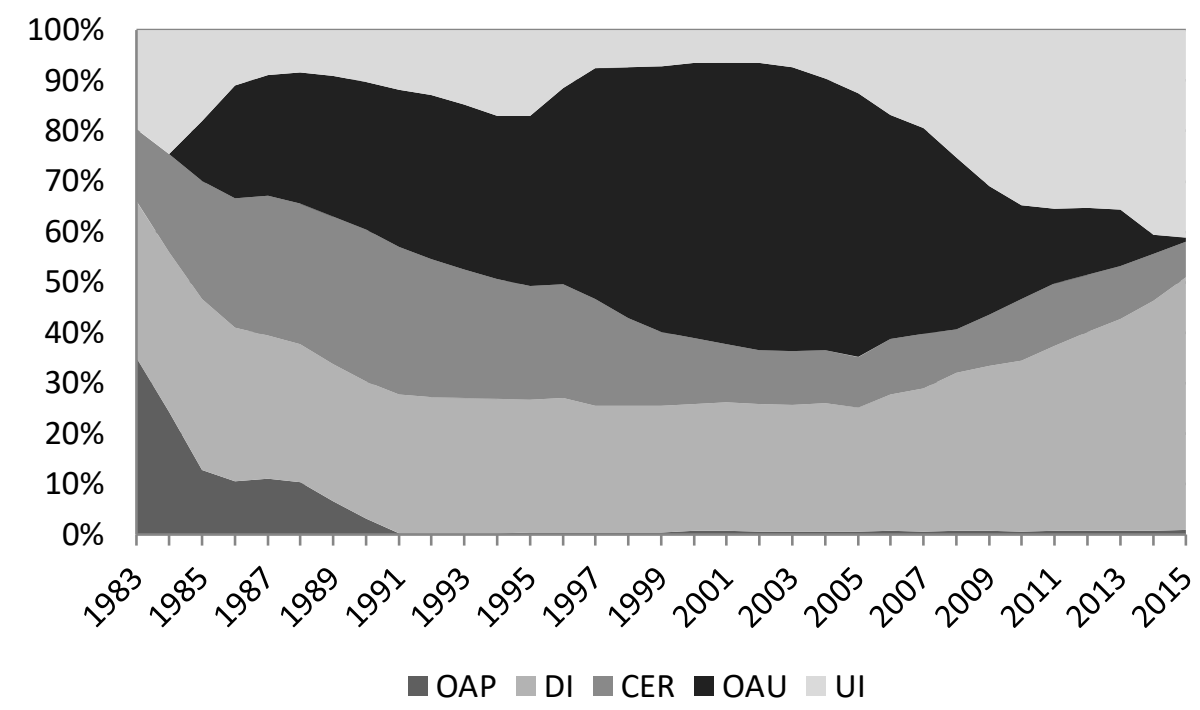

Source: National Pension Office (2019), Office National de l'Emploi (2021), National Institute of Incapacity and Invalidity (2020)

Figure 3.5: Pathways to retirement - women, age 60-64

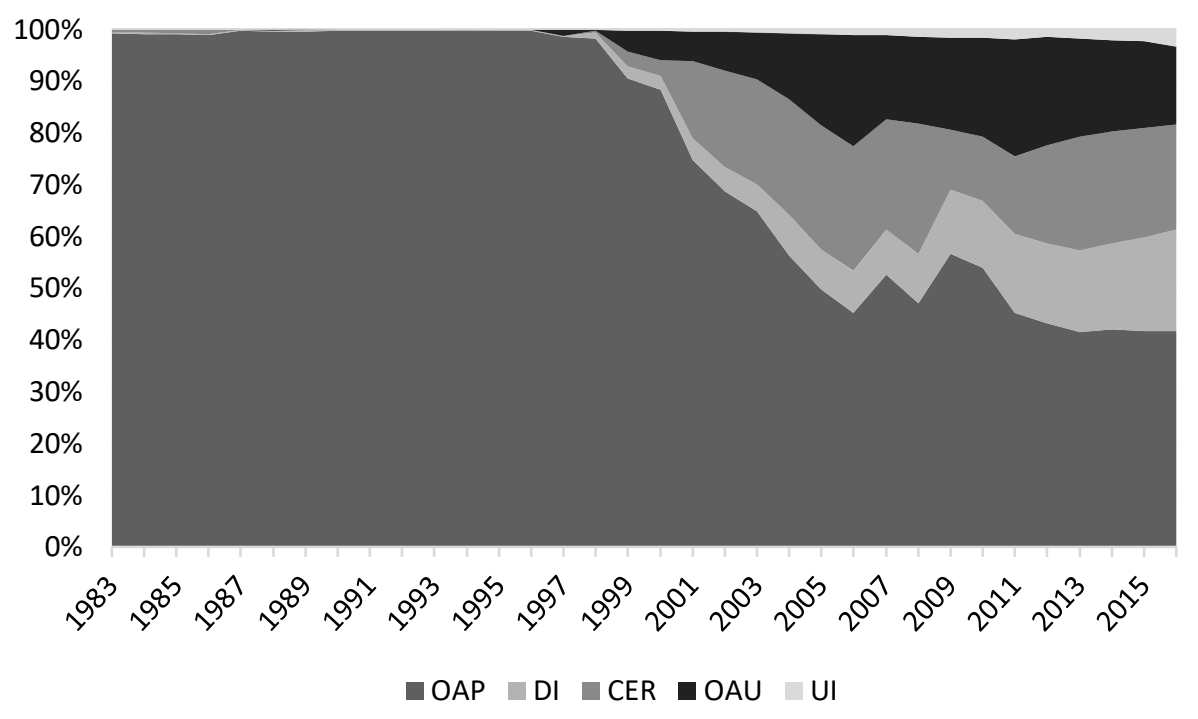

Source: National Pension Office (2019), Office National de l'Emploi (2021), National Institute of Incapacity and Invalidity (2020) 


\subsection{MEASURING RETIREMENT INCENTIVES}

This section describes the simulation model used to quantify the retirement incentives created by the social security system. To observe the effect of changes and reforms over time, calculations are done for the different age cohorts in each year starting in the early 1980 's.

Given the life-cycle dimension of pensions, we define a synthetic earnings history of a cohort through time and scale it to reflect differences in socio-economic status. For each pathway and case, we calculate and aggregate the benefits the representative individual is entitled to at a given age in a given year.

\subsubsection{EARNING HISTORIES}

We consider two scenarios: a common scenario, with key assumptions based on international data used for all countries in the NBER ISS project and a Belgian scenario with assumptions based on Belgian data. We model earnings histories for two types of individuals: single males and single females. We distinguish stylized variants of low, medium and high earners. For each year of observation, each of these 6 cases is associated with specific earnings level, career length and lifetime earnings profiles. We detail the underlying calculations and assumptions below.

First, we use the median income of full-time non-manual wage-earners in 2014 as reported by Statistics Belgium (2016) to define the median worker and attribute this wage to males aged 50 in 2014. We adjust the median wage at the anchoring age of 50 to each simulation year using a fixed deflator, which is the average wage growth rate of wage earners between 1980 and 2016. We then define low earners as persons earning 50 percent of the median and high earners as receiving 200 percent of the median earner's income. We 
assume that female median earnings are 7.6 percent below male median at age 50 for all years of study - with 7.6 percent corresponding to the average hourly wage gap between men and women in 2014 as reported by Statistics Belgium (2016).

Second, we derive earnings histories for these 6 cases in the two scenarios. All earnings histories share a common assumption of no career interruptions - with any variation in career length simply translated into different starting dates.

In the common scenario, we assume that the low earners start working at age 16, the median earners start working at 20 and the high earners start working at 25. Once these career lengths have been determined we derive lifetime earning histories for each case. The common earnings profile uses a time-invariant common synthetic earning profile that is differentiated for men and women and for the three income levels (see figure 3.6). The profiles are calculated with data from the US, Germany and Italy. ${ }^{141}$ Figure 3.6 presents the patterns of the earning profiles - the same for all years concerned - each expressed as a proportion of the anchoring wage at age 50 .

In the Belgian scenario, we shorten the careers of men by 5 years in order to model more incomplete (but more realistic) careers. We further consider an average gap in career length between men and women of 6 years - in line with the results of a report from National Pension Office (2005). This assumption is plausible as the upper age for compulsory schooling has been set at 18 years for several decades, with numerous persons studying well-beyond. Also, other forms of work interruptions are not uncommon. ${ }^{142}$ The Belgian scenario thus assumes that male (female) low earners start

\footnotetext{
${ }^{141}$ See the US chapter of Börsch-Supan and Coile (2021) for details.

${ }_{142}$ Military service obligations for men until the late 1990's also contributed to a later start in the working lives.
} 
working at age 21 (27), male (female) median earners start working at age 25 (31) and male (female) high earners start working at age 30 (36).

In a second step, The Belgian specific earnings profile is built using the average gross monthly conventional wage of non-manual wage earners by age and is time and sexspecific - but its shape does not vary by income level beyond the different starting ages. ${ }^{143}$ Figure 3.7 presents the average of the time-specific Belgian earning profiles, again expressed as a percentage of the wage at age 50. Unlike the common profile - that assumes an important increase of the wage in the first years of the career (see figure 3.6), a decrease in the growth of wages until 50, and constancy thereafter - the Belgian-specific earnings profile displays a continuous and almost constant increase of the wage throughout the career and until age 60. Expressed differently, while the common profile captures an internationally more usual career-wage profile, the Belgian-specific pattern better fits the specificities of the Belgian wage-setting system with its automatic indexing of wages to changes in the Consumer Price Index and collective wage bargaining on wage growth across income levels.

${ }^{143}$ Because of data limitations, the earnings profile is kept constant from 1980 to 1999 and from 2015 to 2016. 


\section{Figure 3.6: Common earnings profiles}
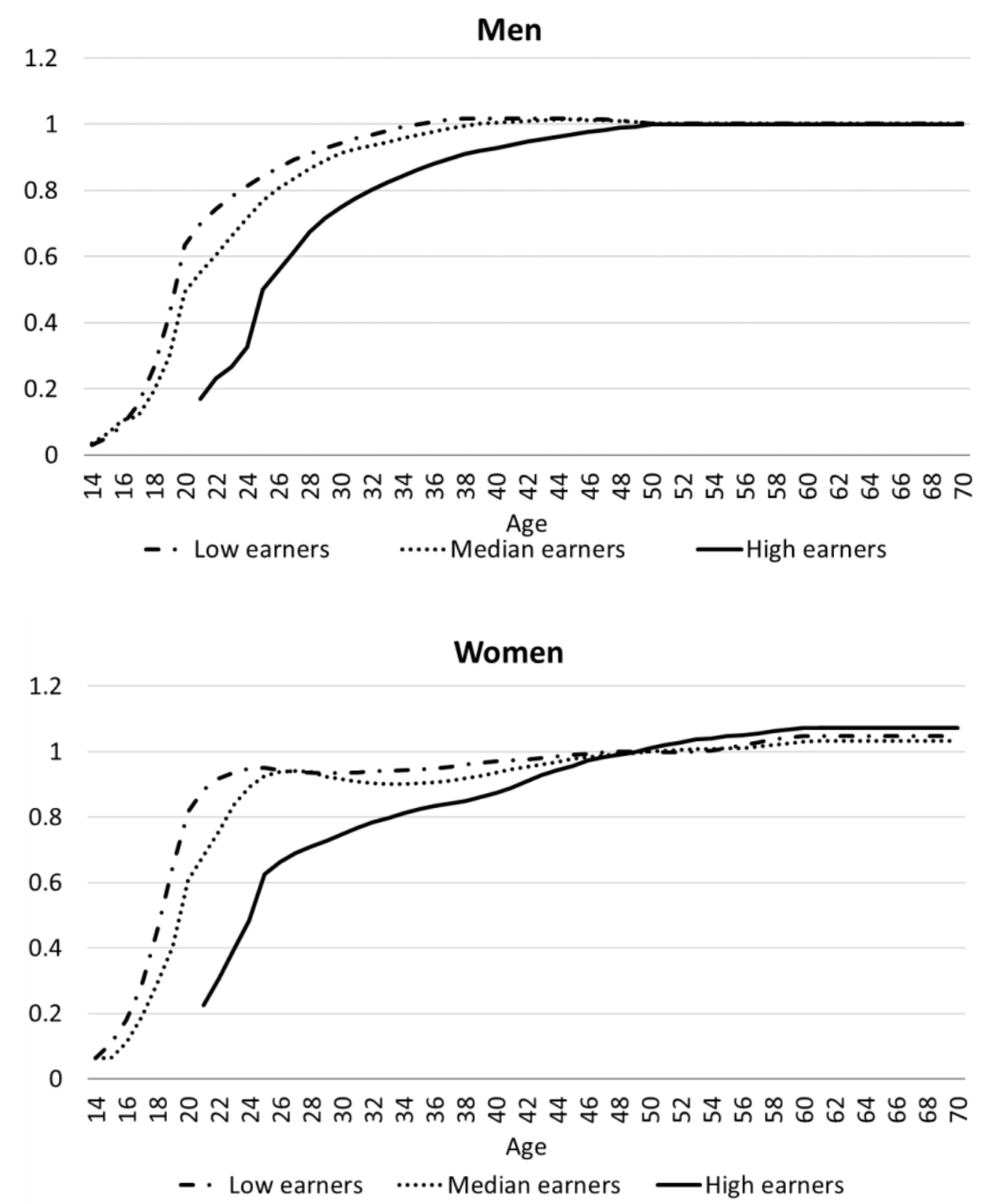

Source: Authors' own calculations based on US Current Population Survey (CPS), German SUF-VSKT 2011 (DRV, 2011) and Italian INPS. 
Figure 3.7: Belgian-specific earnings profiles

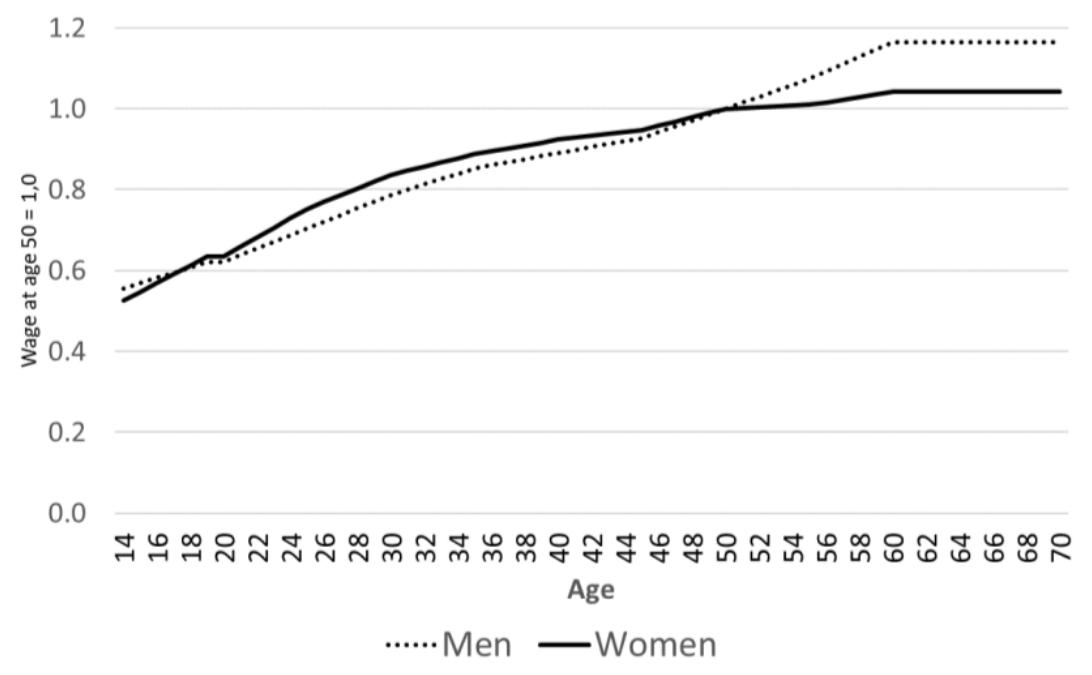

Source: Authors' own calculations using Statistics Belgium ${ }^{144}$

Each scenario is accompanied by an assumption in terms of the applicable tax system. In the common scenario, we use the tax rules applicable in 2016 (in real terms) to all simulation years. The motivation for this approach is to clearly distinguish the pure effects of pension policy from those of general tax policy. ${ }^{145}$ In the Belgian scenario, we apply the tax rules as they were applicable year after year to reflect the changes in the entire taxbenefit landscape as experienced by real-world workers.

\subsubsection{BENEFIT STREAM}

From these earnings profiles, we calculate, for each age-year cohort the after-tax benefit stream from each of the following program: old-age pension, conventional early retirement, unemployment insurance and disability insurance. For an individual $i$, defined according to his or her sex and the level of career earnings, we calculate the after-

\footnotetext{
${ }^{144}$ See 'Salaires mensuels bruts moyens' at https://statbel.fgov.be/fr/themes/emploi-formation/salaires-etcout-de-la-main-doeuvre/salaires-mensuels-bruts-moyens\#figures

145 The tax rates obtained using the 2016 rules are almost identical to those derived by the OECD tax model.
} 
tax benefit $B_{k, t, a}(R, i)$ from the program $k$ in time $\mathrm{t}$ for all ages $a \geq R$, where $R$ is the first year of benefit receipt. Once retired, we assume benefits remain constant in real terms in future years.

We look at retirement ages ranging from 55 to 65 from year 1980 to 2016. Our simulation thus considers any potential cohort differences and changes thereto, as well as transitory and permanent changes over time (index $t$ ) and as a function of age (index $a$ ). The lack of eligibility for pathway $\mathrm{k}$ at an age $a$ at time $t$ conditional on retirement at $R$ is modeled by setting $B_{k, t, a}(R, i)$ equal to 0 .

The simulation model allows for a rich set of scenarios in terms of individual characteristics. Also, it allows for simulations of counterfactuals and system reforms. For example, the common scenario of section 3.4 relies on a counterfactual assumption that only benefit rules have changed but no tax changes have occurred during the entire period of analysis. The motivation for this deviation from the empirically observed institutional setting is to separate out strictly retirement-related changes from broader tax policy changes affecting the wider population. The Belgian scenario of section 3.5 explores the role of tax policy changes, and provides also counterfactual analysis of benefits indexation rules and thresholds.

\subsubsection{SOCIAL SECURITY WEALTH, ACCRUAL AND IMPLICIT TAX}

From the benefits, we calculate different indicators of the incentives created by the social security system. The key concept is the annual accrual of social security wealth. The SSW is the present discounted value of all future benefit flows from a given social security program for a given individual at a given age in a given year. 
SSW for an individual of type $i$ starting to claim benefits from program $k$ at age $R$ in time $t$ is then given by:

$$
S S W_{k, t}(R, i)=B_{k, t, R} \sum_{a=R}^{T} \sigma_{i, a} \beta^{a-R}
$$

Where $B_{k, t, R}$ is the after-tax benefit from pathway $k$ at age $R$ as calculated above. The formula sums up these benefits until the end of life T. Discounting is done allowing both for time preference and mortality adjustments: $\sigma_{i, a}$ is the survival probability ${ }^{146}$ at age $a$ for individual $i$ and $\beta$ is the time discount rate that we assume to be equal to 3 percent real. Since we assume real constant benefit once in the program, the amount stays the same in the forthcoming years if the person retires on old-age pension. In the case of an exit through unemployment, conventional early retirement or disability, the benefits change according to the age since at the statutory eligibility age the individual starts to receive old-age pension benefits instead of the other benefits - essentially splitting the right hand-side of the formula in two separate sums before and after the statutory eligibility age.

Based on this SSW, we then compute a secondary incentive measure that represents the variation in SSW that is obtained by retiring one year later. Postponing claiming by one year has two effects on social security wealth. On the one hand, annual benefits $B_{k, t, a}(R, i)$ can vary with later claiming due to additional earnings entering the benefit formula (and possible actuarial adjustments). On the other hand, however, benefits are received one year later.

\footnotetext{
${ }^{146}$ Obtained from the average survival rates of the EU28 provided by Eurostat.
} 
The accrual of social security wealth is then given by:

$$
A C C_{k, t}(R, i)=S S W_{k, t+1}(R+1, i)-S S W_{k, t}(R, i)
$$

The accrual can thus be positive, zero, or negative. If the accrual is negative, the social security system imposes an implicit tax on working longer. The implicit tax rate is the (negative) accrual of social security wealth divided by the after tax earnings during the additional year of work $Y_{\mathrm{t}+1, \mathrm{I}}$ :

$$
\operatorname{ITAX}_{k, t}(R, i)=\frac{-A C C_{k, t}(R, i)}{Y_{t+1, i}}
$$

This last measure shows the tax on continued activity from retiring one year later. A positive value means that there is a tax on working longer, a negative value represents a subsidy for working longer.

\subsection{RETIREMENT INCENTIVES: COMMON PROFILE}

The present section presents the analysis of the incentive measures defined in the previous section for the 6 "common scenario" cases by sex and income level across time. To simplify the presentation, the general discussion of incentives at the level of the individual focuses on median earner profiles in the context of the old-age pension program. We only present ITAX measures for other benefit programs as they capture the essence of the incentive landscape. When discussing the impact of these individual incentive indicators for aggregate outcomes, all incentive measures are summed across programs and across earnings levels to one representative measure that is confronted with observed employment rates. 


\subsubsection{INCENTIVES BY PATHWAY}

In figures 3.8 to 3.11 , we present the incentive measures for the old-age pension scheme for the median earners. Results are shown for both sexes and two age groups: age 55-59 and age 60-64.

For men aged 55-59, figure 3.8 displays very marked changes in the SSW. The SSW of median earners displays a generally increasing trend over the years thanks to the discretionary increases of ceilings for pensionable earnings ${ }^{147}$. Without such discretionary increases, the SSW would be flat - reflecting the crucial role played by the floors and ceilings in the system. ${ }^{148}$ This is particularly important in the early eighties when the ITAX is increasing rapidly. This is the -result of the combination of both upward changes of ceilings that are more important than in other years and the higher growth rate of median income. Moreover, given that workers receive a benefit of zero before they reach the early eligibility age of 60 , the ITAX is negative for every year.

The ensuing discrete jump of the SSW curves after 1990 can be attributed to the end of the pension penalty of 5 percent for exits before the statutory eligibility age ${ }^{149}$. This translates in a large peak of the accrual and the corresponding drop in the implicit tax as a result of the discrete jump in entitlements for all individuals claiming benefits at the early eligibility age - as is the case of individuals quitting the labor market between ages 55 and 59.

As mentioned in chapter two, the early eligibility age of both men and women gradually increased from 60 to 63 between 2013 and 2018. In our simulation, we consider that if a

\footnotetext{
147 See chapter two for more information on ceilings for pensionable earnings.

148 Implicitly, the discretionary increases - imperfectly - mimic wage-indexing of past earnings.

149 See chapter two for more information on the pension penalty.
} 
worker exits the labor market through the old-age pension before the early eligibility age, he/she receives a benefit of zero until he/she reaches the early eligibility age. Since median earners in the common scenario do not satisfy the long-career exception, they are affected by the reform ${ }^{150}$. Thus, an increase in early eligibility age translates into a decrease of the SSW because the worker starts receiving pension benefits one year later. Therefore, the downward trend in SSW as of 2013 is attributable to the increase in the early eligibility age and the associated career requirements. Accordingly, the ITAX at age 59 decreases in 2013, because there is an incentive for the worker aged 59 to stay on the labor force until 61 because the early eligibility age rises to 61 in 2014.

Figure 3.8: Incentive measures - Old-age pension - men median earners, ages 55-59

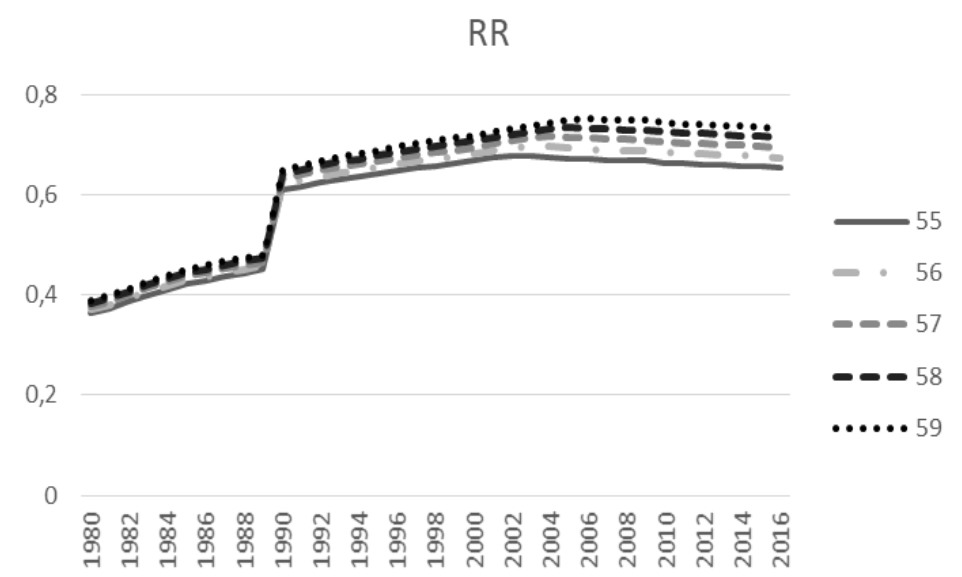

\footnotetext{
${ }^{150}$ See chapter two for the historical evolution of the statutory and early eligibility ages of the old-age pension and the associated career requirements.
} 
SSW
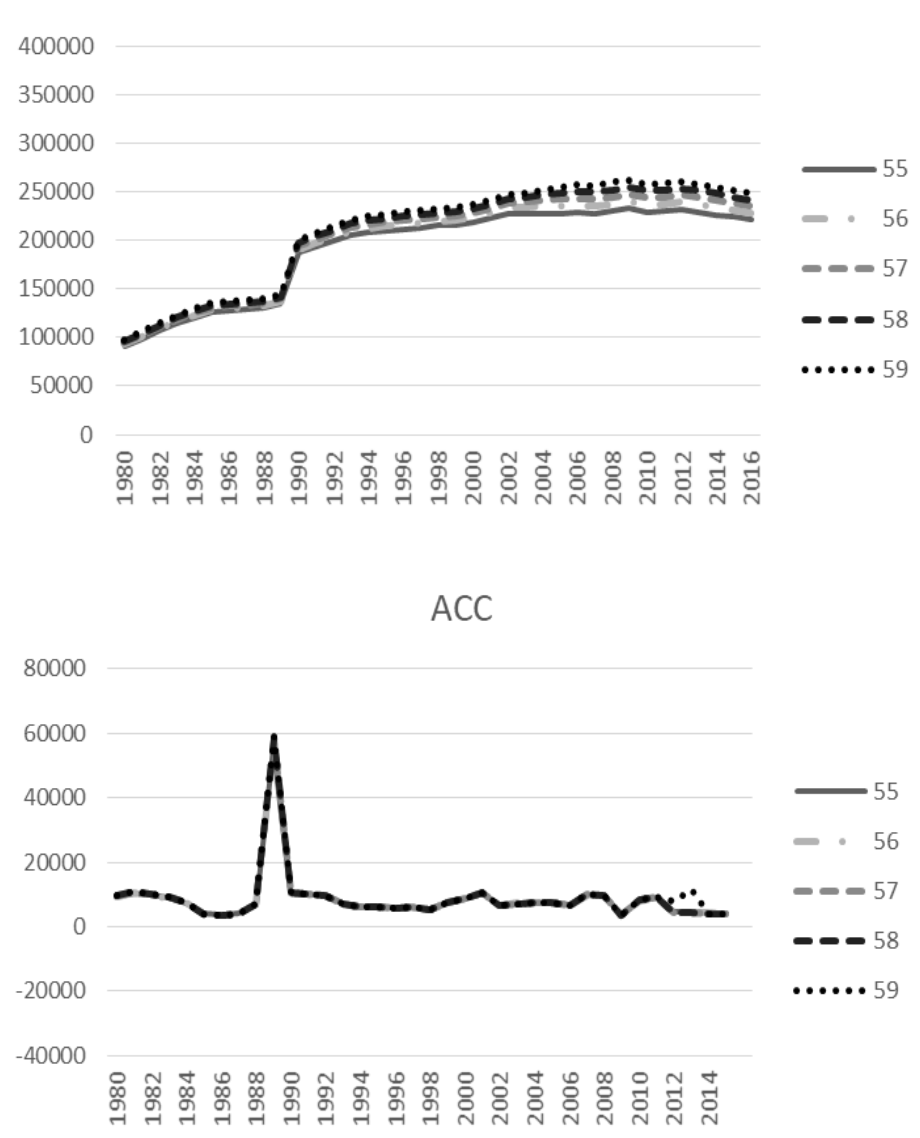

ITAX

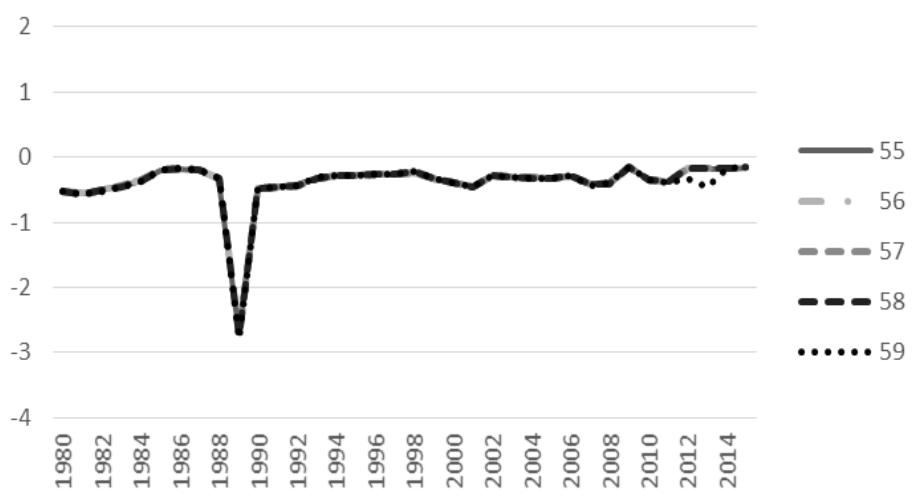

Source: Authors' calculations

Note: RR, SSW, ACC and ITAX correspond to the replacement rate, the social security wealth, the accrual and the implicit tax rate respectively. 
Figure 3.9 reports the social security incentive measures for female median earners retiring between ages 55 and 59. The results are similar to those of men except for the reform of 1987, which increased the early eligibility age from 55 to 60, adding a sharp discontinuity by replacing every potential year of benefits before 60 by zero. Moreover, as the female statutory eligibility age was already set at 60 before 1997, women were not directly impacted by the end of pension penalty in 1991. In addition, other assumptions play out: as women are assumed to have the same career length as men with and only slightly lower median earnings, their higher life expectancies and shorter full career conditions (until 2009 only) lead them to have a significantly higher SSW. This effect is partially cancelled out between 1997 and 2009 because of the increase in full career conditions from 40 to 45 years. The resulting decrease in SSW during the latter period leads to an increased incentive to leave the labor market before each increase in career condition, and thus a positive ITAX at each step.

Figure 3.9: Incentive measures - Old-age pension - women median earners, ages 55-59

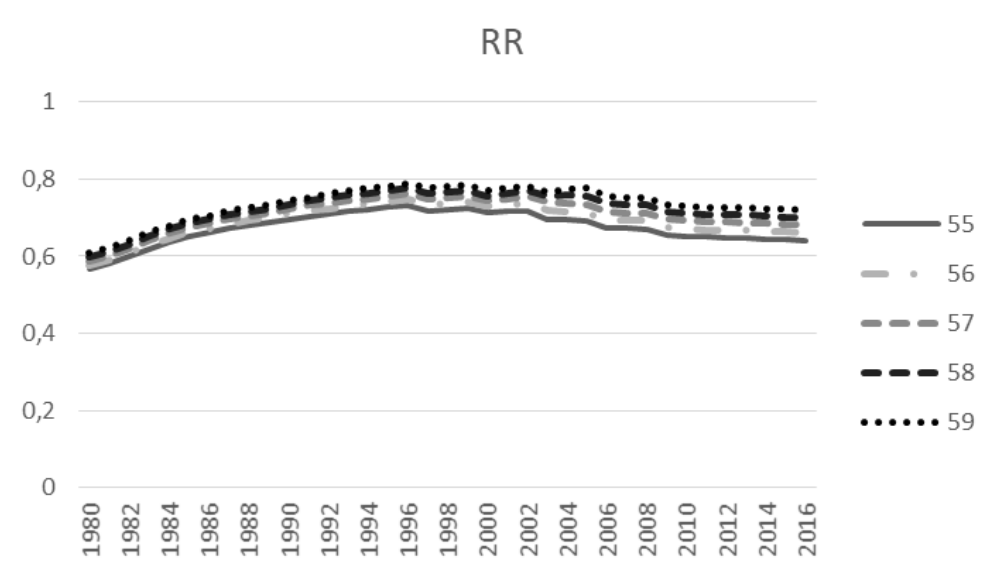


SSW
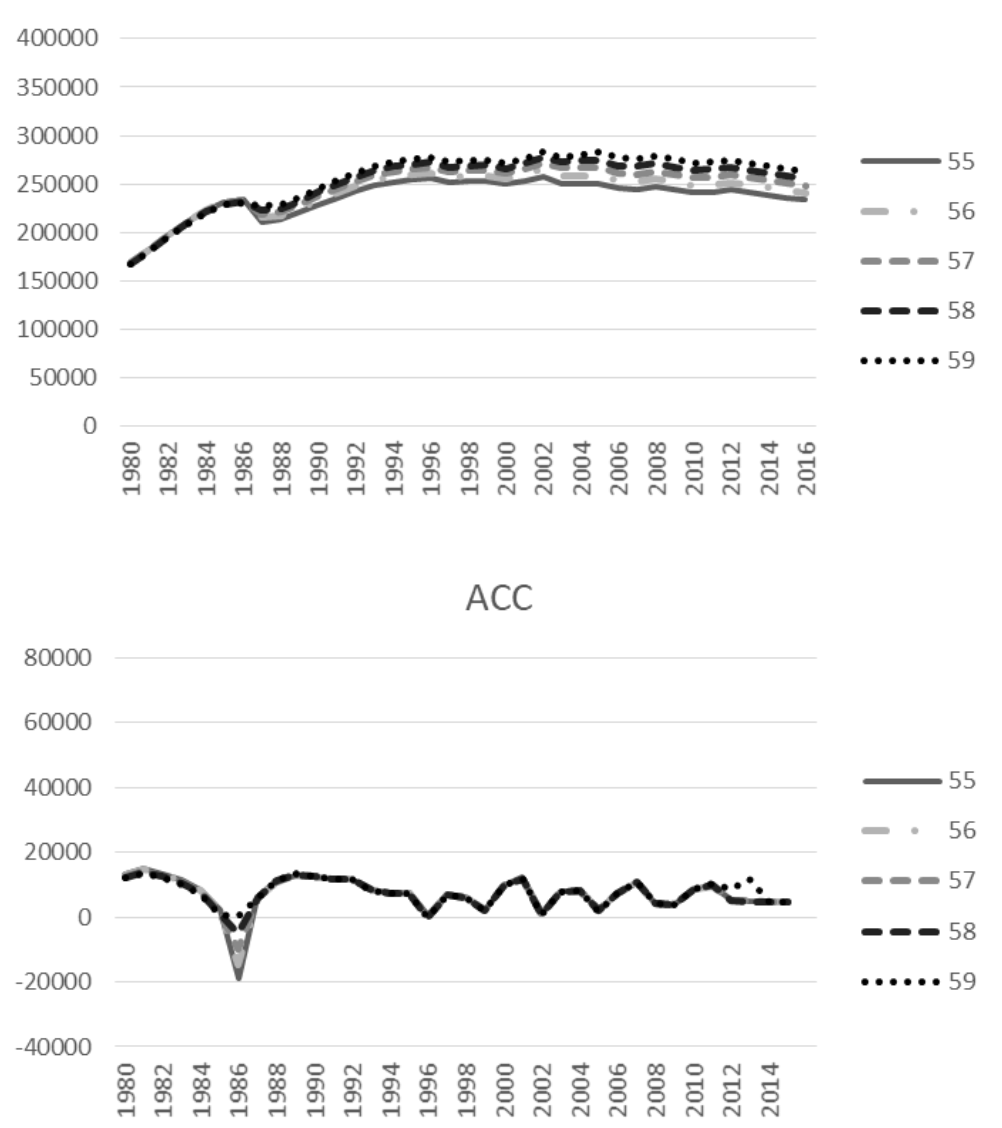

ITAX

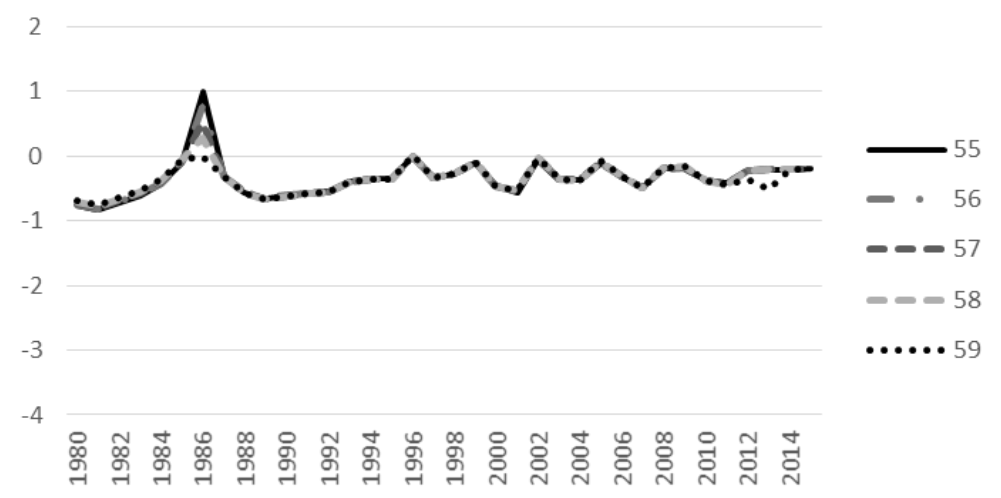

Source: Authors' calculations

Note: RR, SSW, ACC and ITAX correspond to the replacement rate, the social security wealth, the accrual and the implicit tax rate respectively. 
Figure 3.10 reports the social security incentive measures for men who retire between the ages of 60 and 64 . The end of the pension penalty in 1991 is still visible but the older the person is when he retires, the less important is the jump of SSW because his pension benefits were less impacted by the pension penalty. ${ }^{151}$ In 2007, the flat-rate pension bonus was created for continued work after age 62 (or after 44 years of career). The program was discontinued in 2015, which translates into a fall in the accruals and an increase in the ITAX at ages 63 to 65 in 2014. In this simulation, the receipt of a pension bonus increases the pension to a level which is higher than the health and disability insurance contribution threshold for retirement ages of 65 in 2008 and of 64 and 65 starting from 2009 onwards. This additional health and disability contribution limits the increase in pension benefits, and thus the increase in SSW, due to the pension bonus at age 64 and 65. Since we assume that the median earner starts working at age 20 and works without career interruption, he is eligible for the long-career exception of the old-age pension scheme that allows for retirement at age 60 in 2013 and 2014, instead of 60.5 and 61, respectively. ${ }^{152}$ Thus, in practice, workers would only be affected by the reform if their career was insufficiently long - which is not the case for the common profile. At age 60, the SSW only starts decreasing in 2015 because in 2013 and 2014, median workers still had access to an early eligibility age of 60. In 2015, they fall out of these long career conditions that require 41 years of career to access old-age pension benefits at age 60 and the early eligibility age is now set at 61.5 .

\footnotetext{
${ }^{151}$ In figure 3.8, this is not the case as there is a maximum penalty of 5 years.

152 See chapter two for the historical evolution of the early eligibility age of the old-age pension and associated career requirements.
} 
Figure 3.10: Incentive measures - Old-age pension - men median earners, ages 60-64
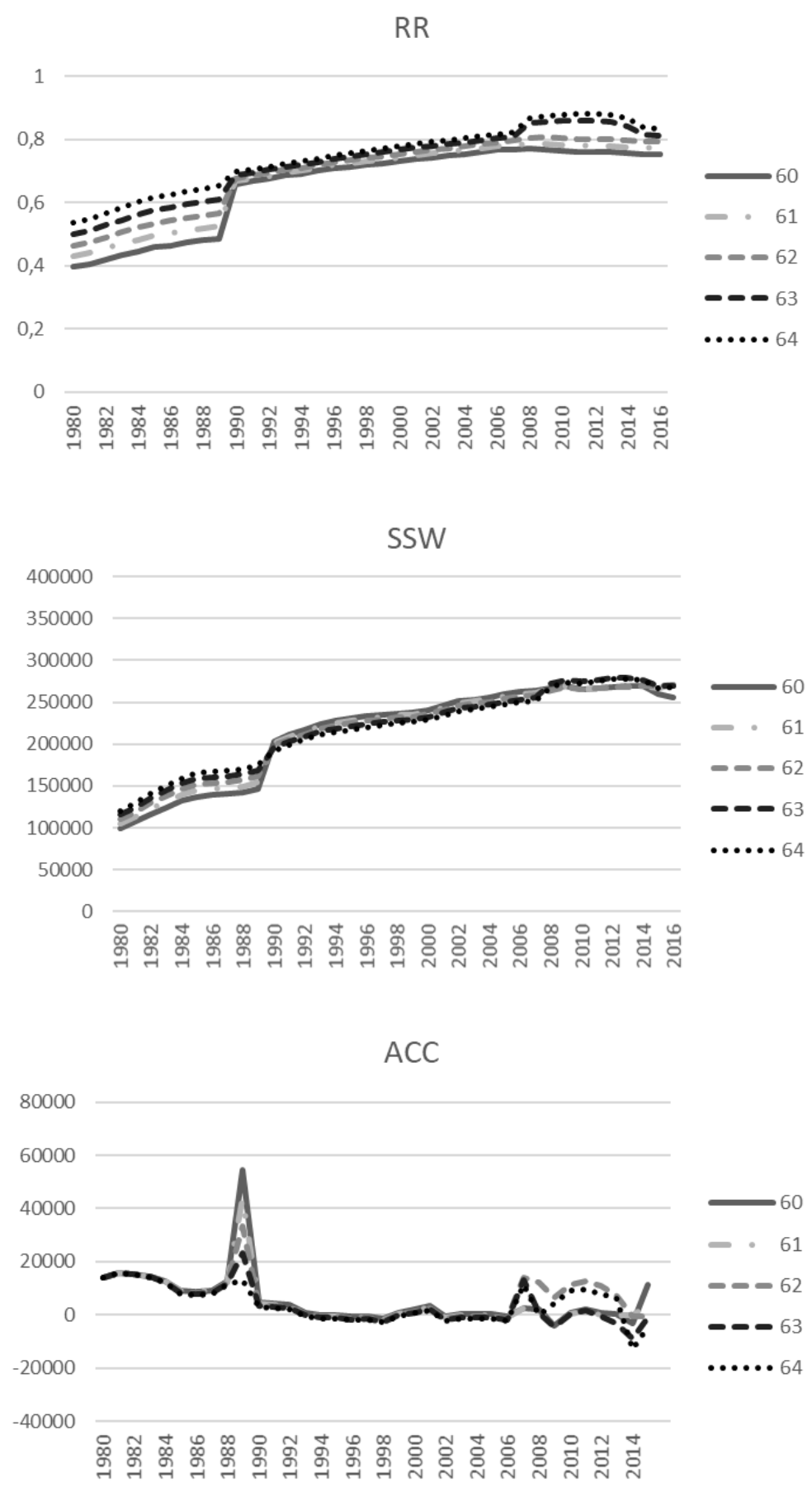
ITAX

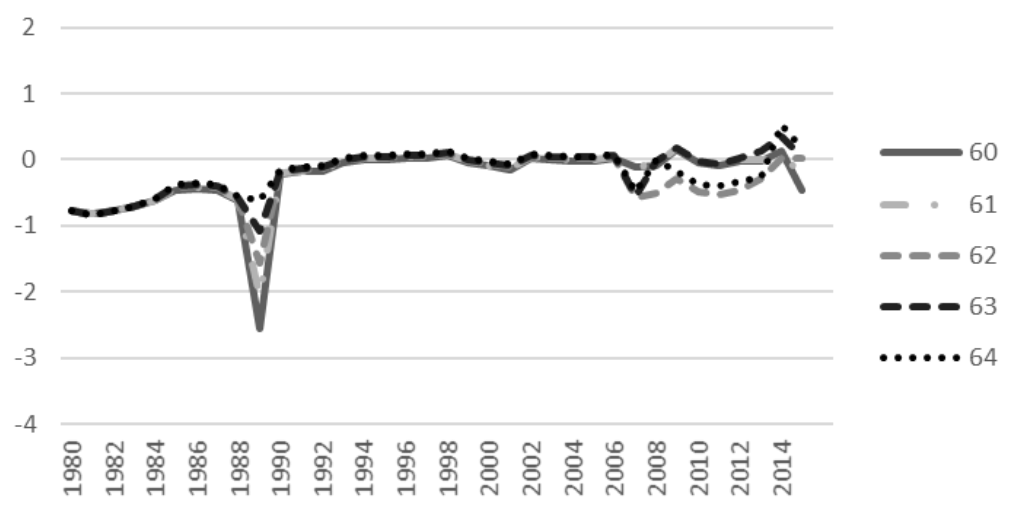

Source: Authors' own calculations

Note: RR, SSW, ACC and ITAX correspond to the replacement rate, the social security wealth, the accrual and the implicit tax rate respectively.

Finally, figure 3.11 depicts the social security incentive measures for women who are median earners and retire between the ages of 60 and 64. Logically, the 1987 reform that increased the early eligibility age of women to 60 has no impact on women who retire after 60. The effect of the increase in full career from 1997 to 2009 and the pension bonus from 2007 to 2015 for retirement ages between 63 and 64 are also visible. Moreover, similarly to men, the receipt of the pension bonus at age 65 also increases the pension to a level higher than the health and disability insurance contribution threshold, which limits the increase in pension benefits. 
Figure 3.11: Incentive measures - Old-age pension - women median earners, ages 60-64
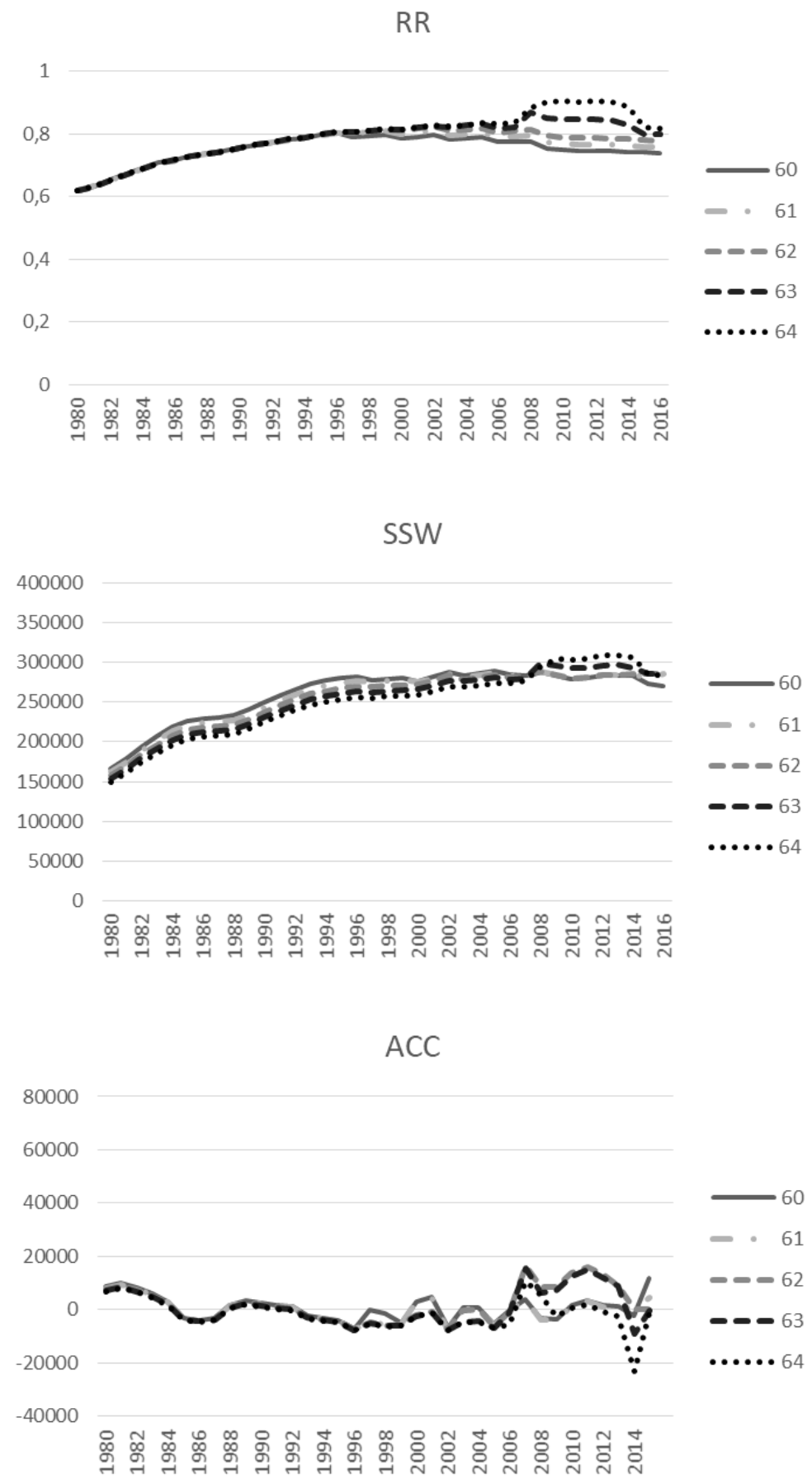


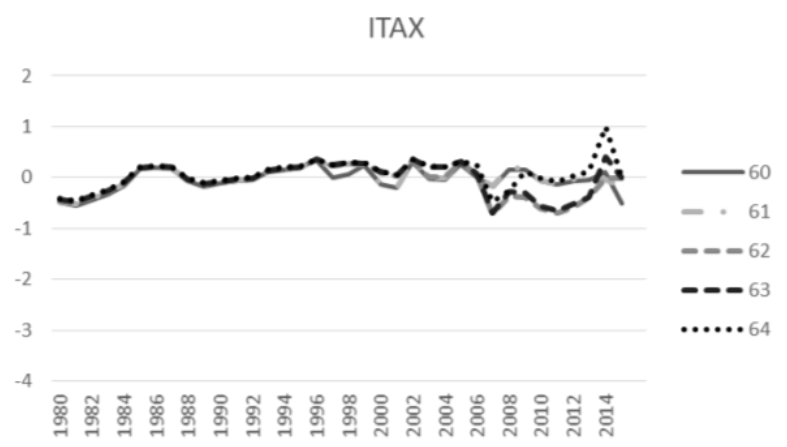

Source: Authors' own calculations.

Note: RR, SSW, ACC and ITAX correspond to the replacement rate, the social security wealth, the accrual and the implicit tax rate respectively.

For the other three pathways, we only present the implicit tax for each sex for reasons of brevity. Similarly, we focus on the age group 55-59 since it is the age-window where most changes in program parameters and employment have happened. Figure 3.12 and Figure 3.13 show the evolution of the ITAX in the conventional early retirement scheme for each age. Contrary to our assumption on the old-age pension, we consider that individuals who lose benefits in the conventional early retirement program would still meet the basic conditions to unemployment benefits - a plausible assumption in the Belgian context. Hence, benefits are not reduced to zero in case of loss of eligibility, but rather replaced by the lower unemployment benefits. The implicit tax for most ages is on average largely positive; this means that there is a strong incentive to exit the labor market through the conventional early retirement. The peak at age 55 in 1986 for both men and women corresponds to the increase of the conventional early retirement eligibility age from 55 to 57. There is an incentive to quit the labor market before the reform takes place in order to be able to access the conventional early retirement pathway at age 55 instead of 57 . The same effect is observed at age 58 in 2008 when the eligibility age increased to 60 . The ITAX at age 56 in 1986 is not impacted by the reform, the worker does not lose access to the conventional early retirement exit pathway if he or she works for one more year. In 1987, the increase in the eligibility to 58 creates a negative ITAX at 57 because the worker now 
has an incentive to stay on the labor market for one more year to have access to the conventional early retirement program rather than exiting at 57 and remaining on lower unemployment benefits until the statutory eligibility age. Thus, from 1987 onwards, the ITAX at 55 and 56 is lower than at other ages because if workers exit before 58 they receive unemployment benefits until the statutory eligibility age. Since the unemployment benefits are less generous than the conventional early retirement benefits, it creates a smaller incentive to leave the labor force. The small drop in 2001 for every age is related to an increase of the median wage that is more important than in previous years and is thus not explained by any changing rules of the system. These changes in the growth of the median wage also explain the small variations we observe between 1987 and 2006 and especially the stiff increase of ITAX in 2009. The same will apply for the results presented below for unemployment and disability programs. In 2008, the increase of the headline entitlement age for conventional early retirement to 60 translates into an double effect: an increase in the ITAX at 57 resulting in an incentive to leave the labor force before the reform; and a decrease in the ITAX at 59 resulting in an incentive to stay on the labor market for one more year to receive conventional early retirement rather than unemployment benefits until the statutory eligibility age. ${ }^{153}$ In 2015, the headline eligibility age increases to 62 and so does the ITAX at age 59.

\footnotetext{
${ }^{153}$ Notice that the impact of the 2008 reform on the ITAX at 59 is less pronounced than the impact of the 1987 reform on the ITAX at 57 simply because the period during which conventional early retirement benefits are replaced by unemployment benefits is longer.
} 
Figure 3.12: Implicit tax of conventional early retirement benefits - men median earners, ages $55-59$

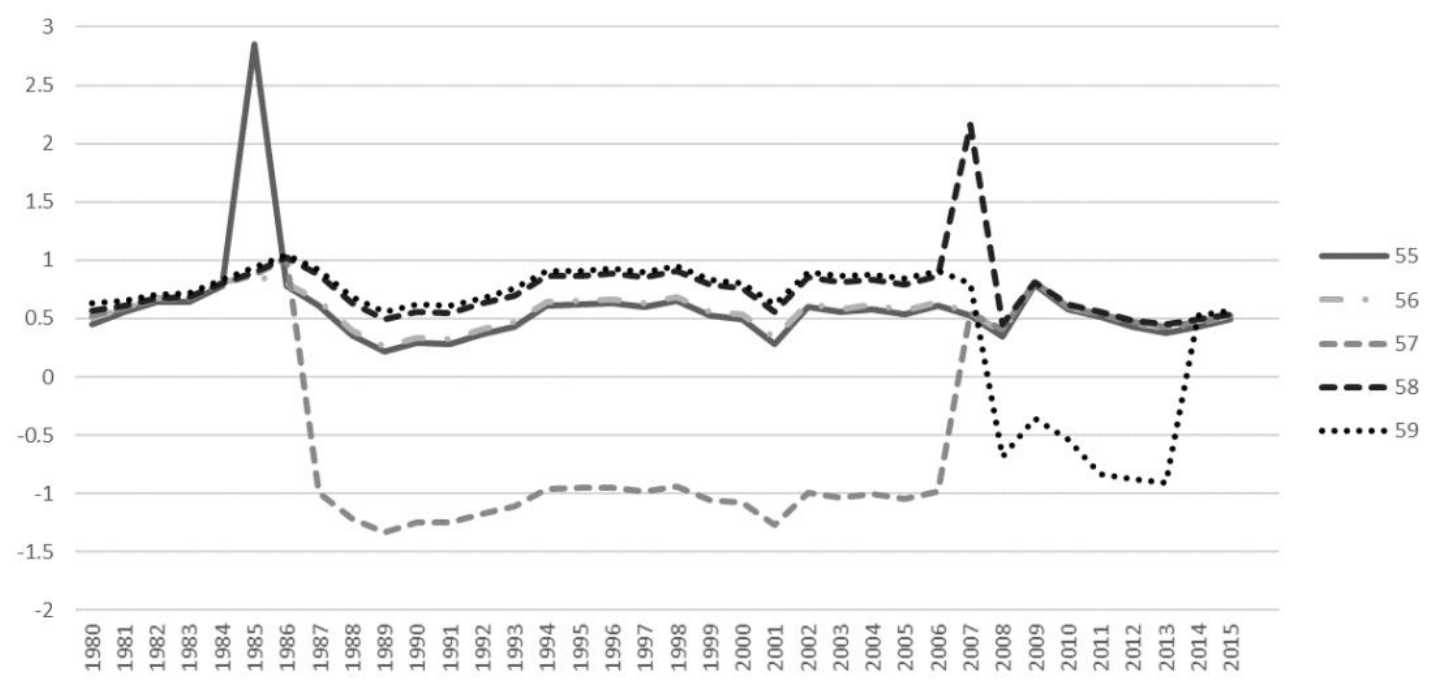

Source: Authors' own calculations

From 2012 onwards, and for exits before age 59, longer periods on conventional early retirement are no longer fully credited at the last observed wage before entering the conventional early retirement program but rather at the legal minimum wage ${ }^{154}$. Though the eligibility age for the conventional early retirement is higher than 59 in the headline regime- the conventional early retirement pathway still is affected. The reason is that exits through unemployment as a fall back option remain possible, with unemployment benefits crediting in the earnings history effectively facing the same limitations as conventional early retirement benefits after the first year of unemployment. Therefore, workers in the headline regime are affected by the reform through the indirect channel of a decrease in the value of unemployment periods in their earnings history whereas those in special conventional early retirement programs (that allows for earlier exit) are directly affected - ultimately to the same effect. As a result, the reform of credited earnings in 2012

${ }^{154}$ See chapter two for more details. 
for long periods of inactivity has led to a decrease of the ITAX at every age from 2012 onwards.

Figure 3.13 illustrates the evolution of the conventional early retirement ITAX for women. The impact of the gradual increase of the statutory eligibility age in old-age pension from 1997 to 2009 is particularly visible. Each stepwise increase in statutory eligibility age translates into an immediate decrease in the ITAX because women receive one more year of conventional early retirement benefits before they are transferred into the old-age pension system. Therefore, an incentive to stay at work for one more year appears at each step.

Figure 3.13: Implicit tax of conventional early retirement benefits - women median

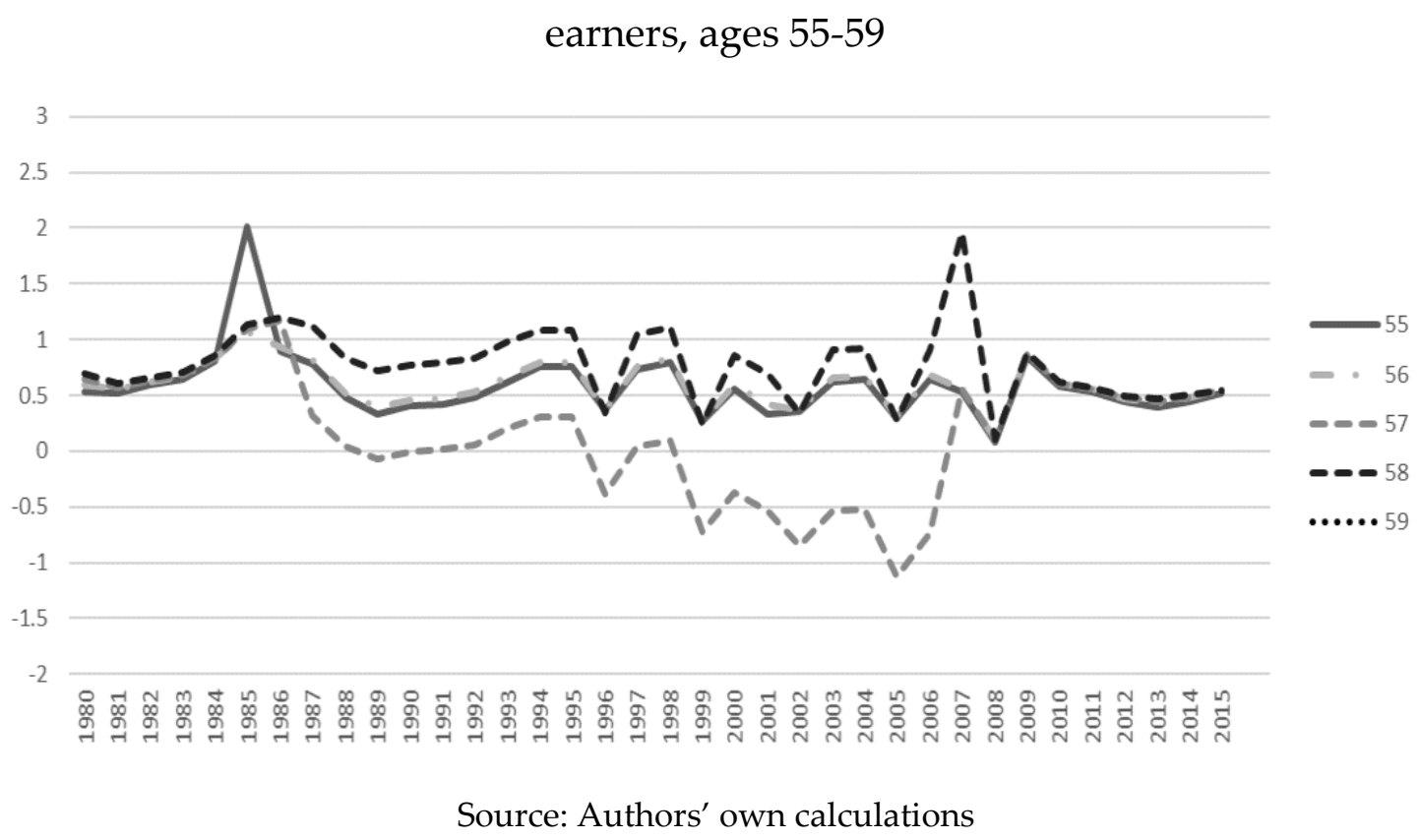

The implicit tax for unemployment also displays some changes, as illustrated in figures 3.14 and 3.15. In 1985, the ITAX decreases sharply in a one-off fashion because of the introduction of the seniority supplement: there is an incentive to wait one more year in order to be eligible for the supplement rather than merely regular unemployment 
benefits. This effect no longer plays out with the same acuity in the following years, where only the increased generosity of the system leaves its mark through a higher implicit tax rate. In 2015, the increase in ITAX at each age is the mirror image of that in 1985: namely the end of the supplement for seniority. For women, the increase of the old-age pension statutory eligibility age also translates into a markedly different incentive pattern at the conventional early retirement level.

Figure 3.14: Implicit tax of unemployment benefits - men median earners, ages 55-59

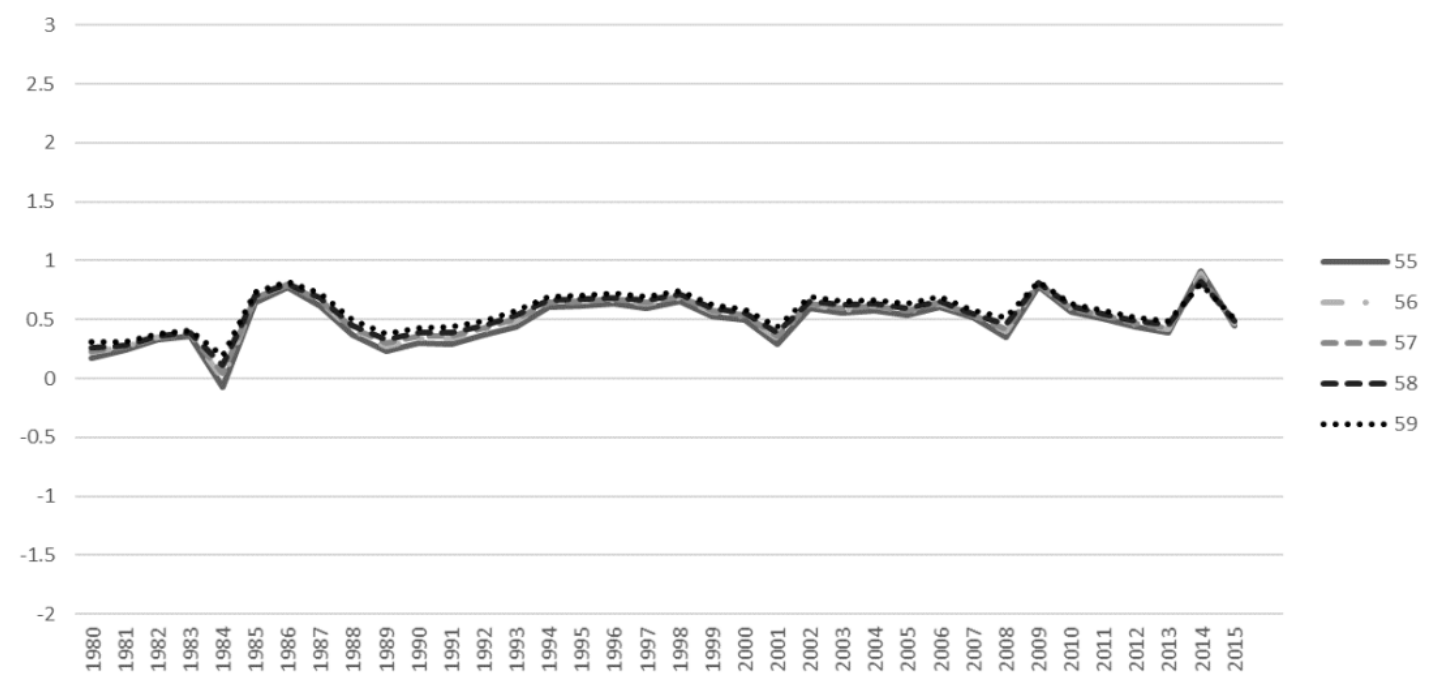

Source: Authors' own calculations 
Figure 3.15: Implicit tax of unemployment benefits - women median earners, ages 55-59

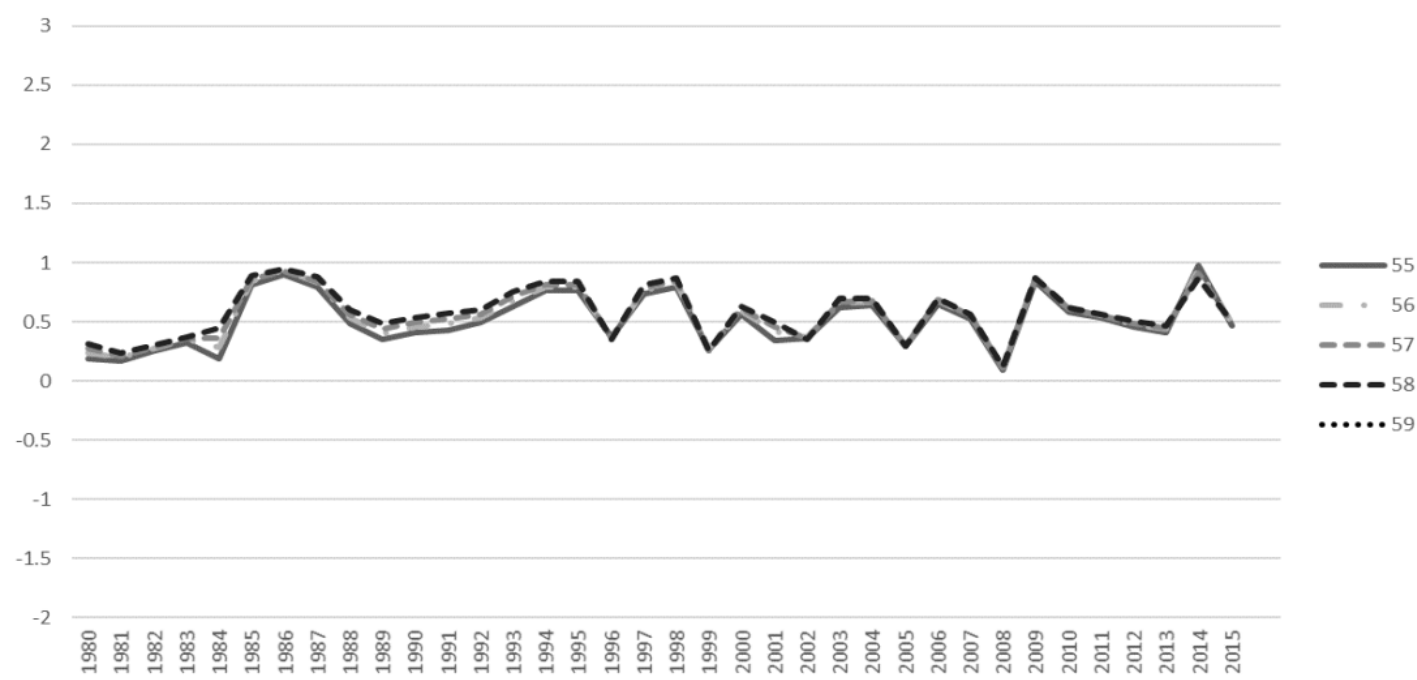

Source: Authors' own calculations

Interestingly, as we already noted in chapter two, there has been little to no change in the benefit calculation in the disability program and the statutory incentives to retire have not been impacted beyond the obvious effect of the increase in the statutory eligibility age for women and the discretionary increases in ceilings for pensionable earnings ${ }^{155}$ (figures 3.16 and 3.17). As mentioned before, and short of information of implementation rules at the level of the institutions managing the disability system, these incentive measures only capture changes in the laws and leave aside changes that could have arisen because of modified implementation over time.

155 The most notable effect is the important discretionary increase in the ceiling of pensionable earnings in 1981. 
Figure 3.16: Implicit tax of disability benefits - men median earners, ages 55-59

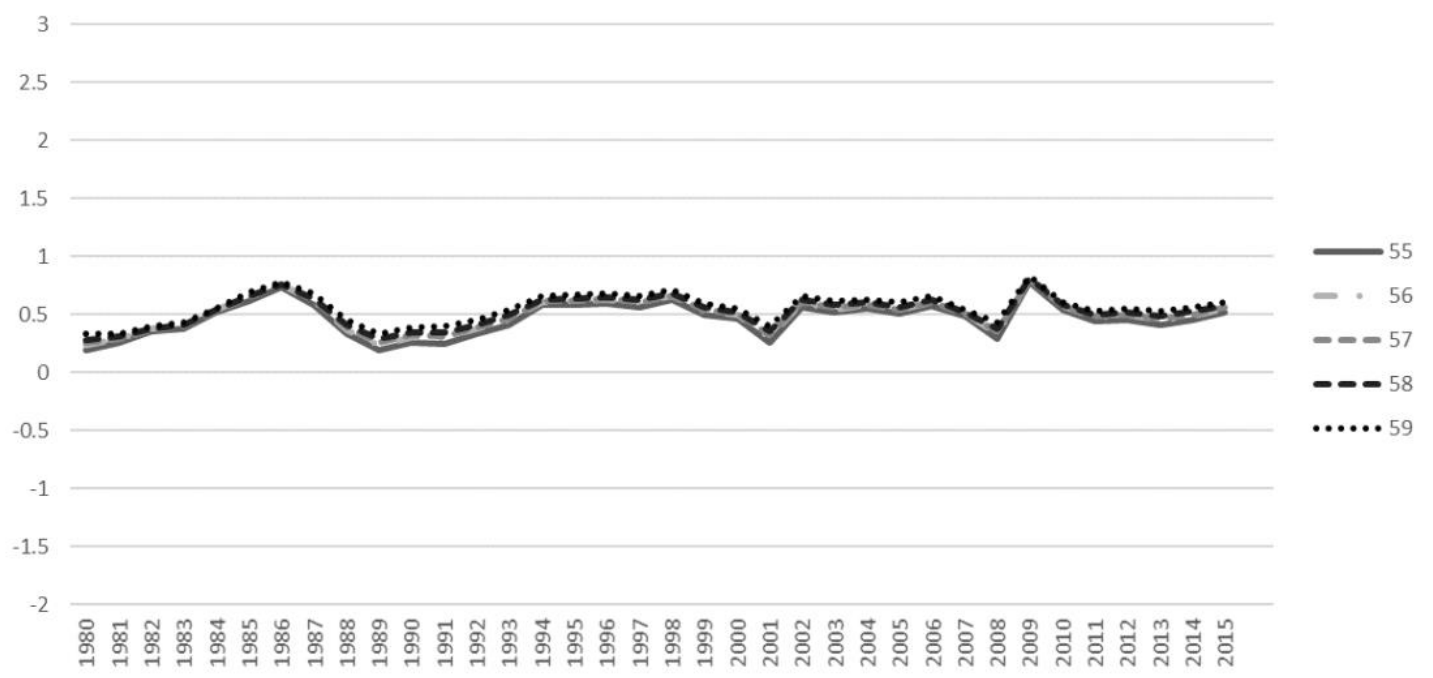

Source: Authors' own calculations

Figure 3.17: Implicit tax of disability benefits - women median earners, age 55-59

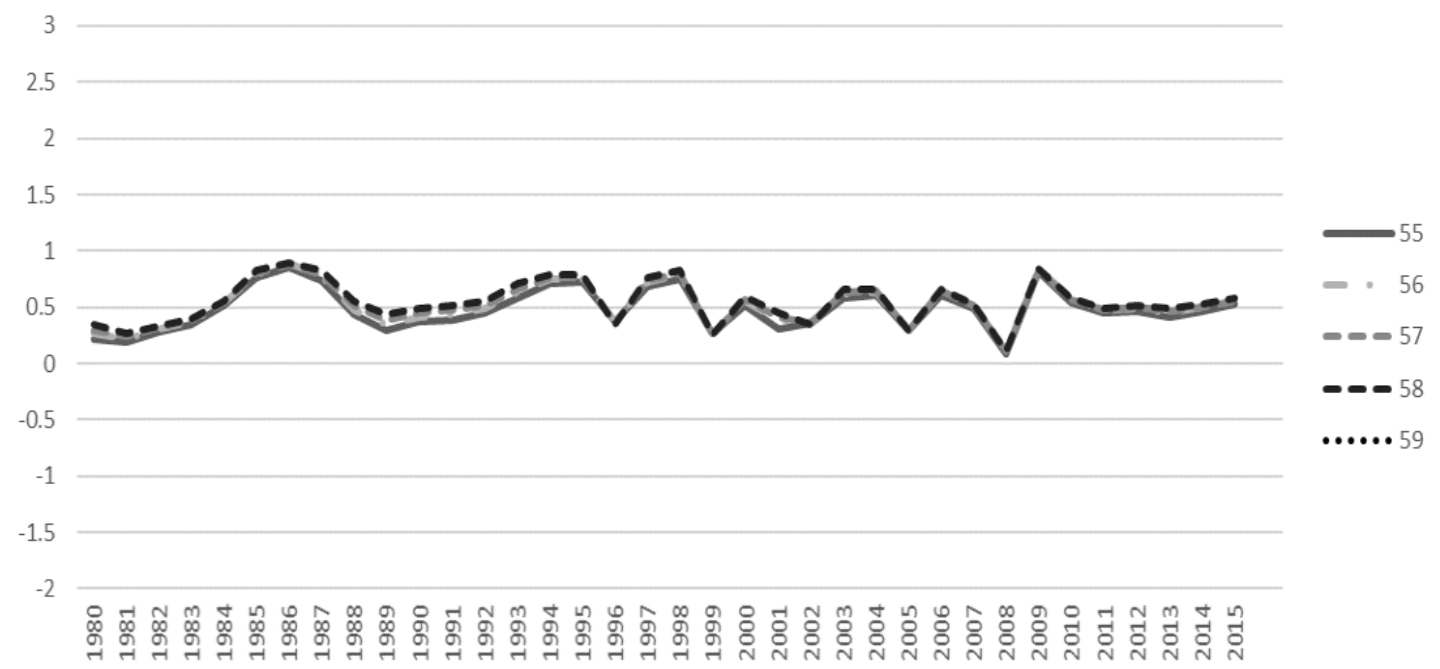

Source: Authors' own calculations 


\subsubsection{AGGREGATE INCENTIVES AND EMPLOYMENT}

To confront the stylized individual incentives with aggregate employment rates, a summary incentive indicator is derived. We rely on the administrative data of figures 3.2 to 3.5 to calculate path-specific weights corresponding to the share of the population that is receiving either unemployment, conventional early retirement or disability benefits. The old-age pension takes the residual weight such that the sum of the weights is equal to one. These weights are obtained by year, age group (55-59 and 60-64), and gender. Finally, incentives are aggregated across income levels.

Figures 3.18 to 3.21 present the results of these aggregate incentive measures for median earners by age, year and sex and include the major reforms that have influenced them since the eighties. Aggregate retirement incentives are heavily influenced by the default option, namely the old-age pension scheme. This is unsurprising as this scheme represents the largest weight (see figures 3.2-3.5) and also affects the benefit that is payable for the longest period of time (from early or statutory eligibility ages until death). Because of the high participation rate of men in the conventional early retirement scheme, this program also has a non-negligible influence on the aggregated ITAX of men for both age groups. 
Figure 3.18: Implicit tax for male median earners by age (55-59)

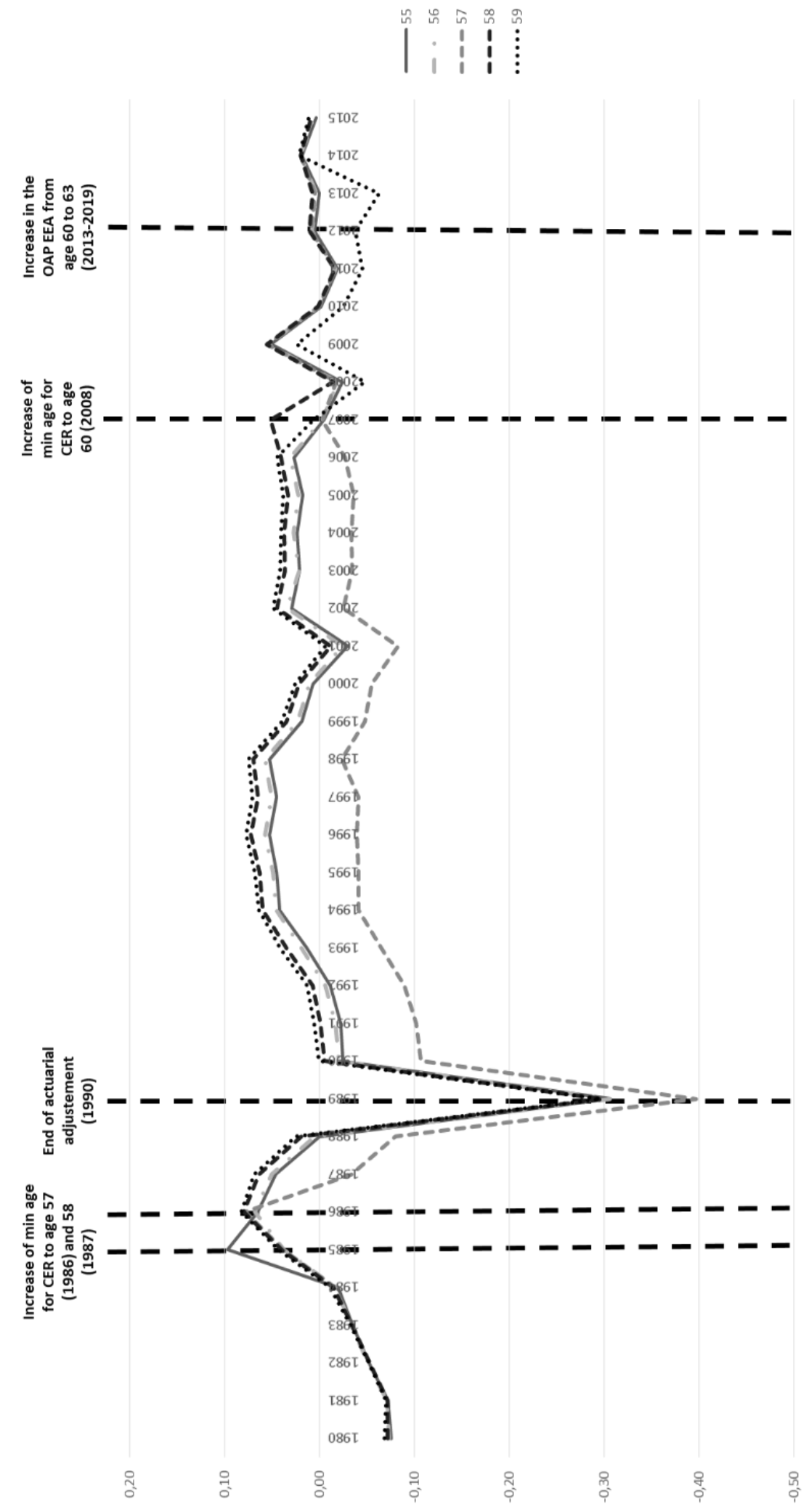

Source: Authors' own calculation 
Figure 3.19: Implicit tax for female median earners by age (55-59)

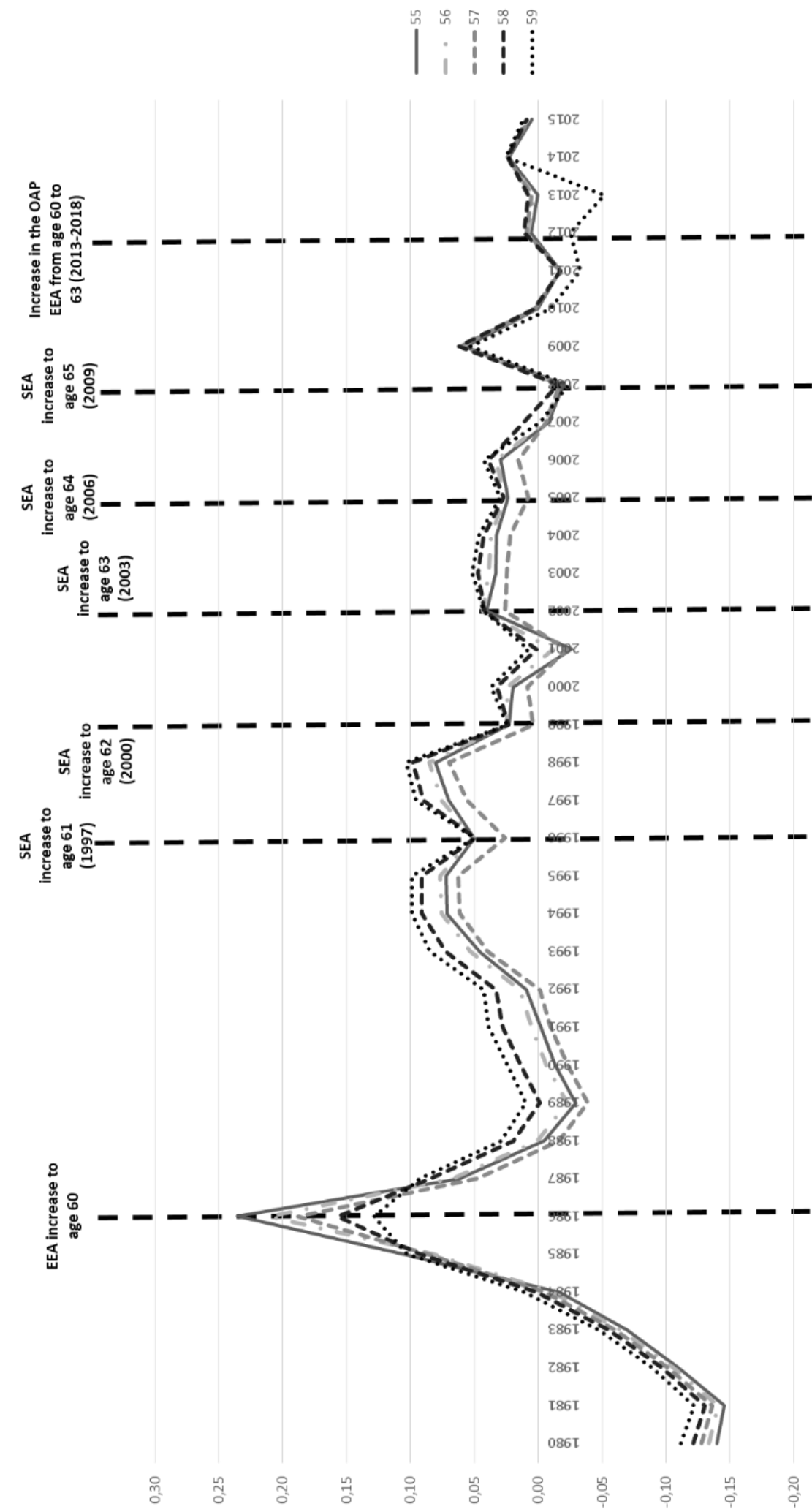

Source: Authors' own calculations 
Figure 3.20: Implicit tax for male median earners by age (60-64)

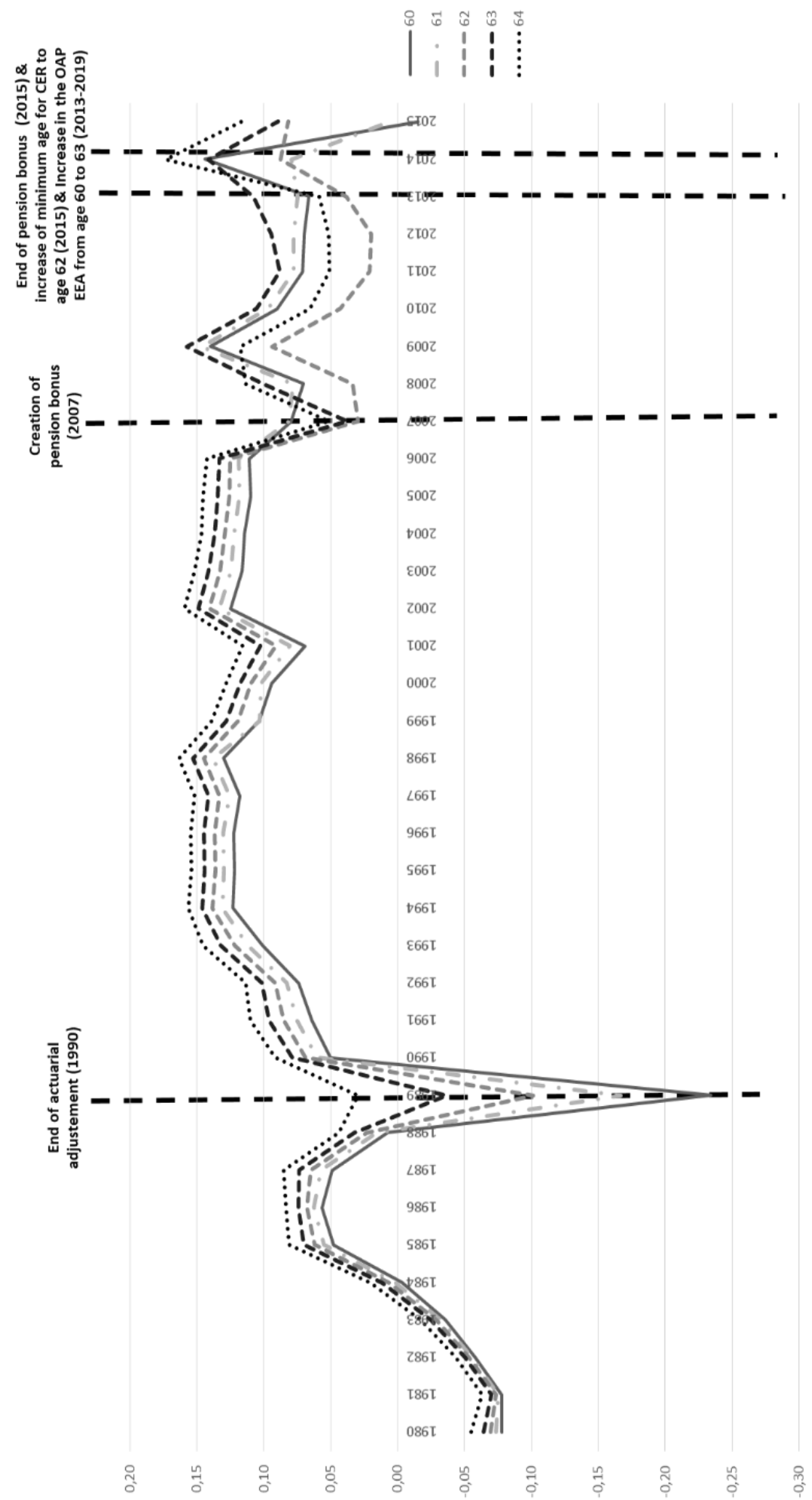

Source: Authors' own calculations 
Figure 3.21: Implicit tax for female median earners by age (60-64)

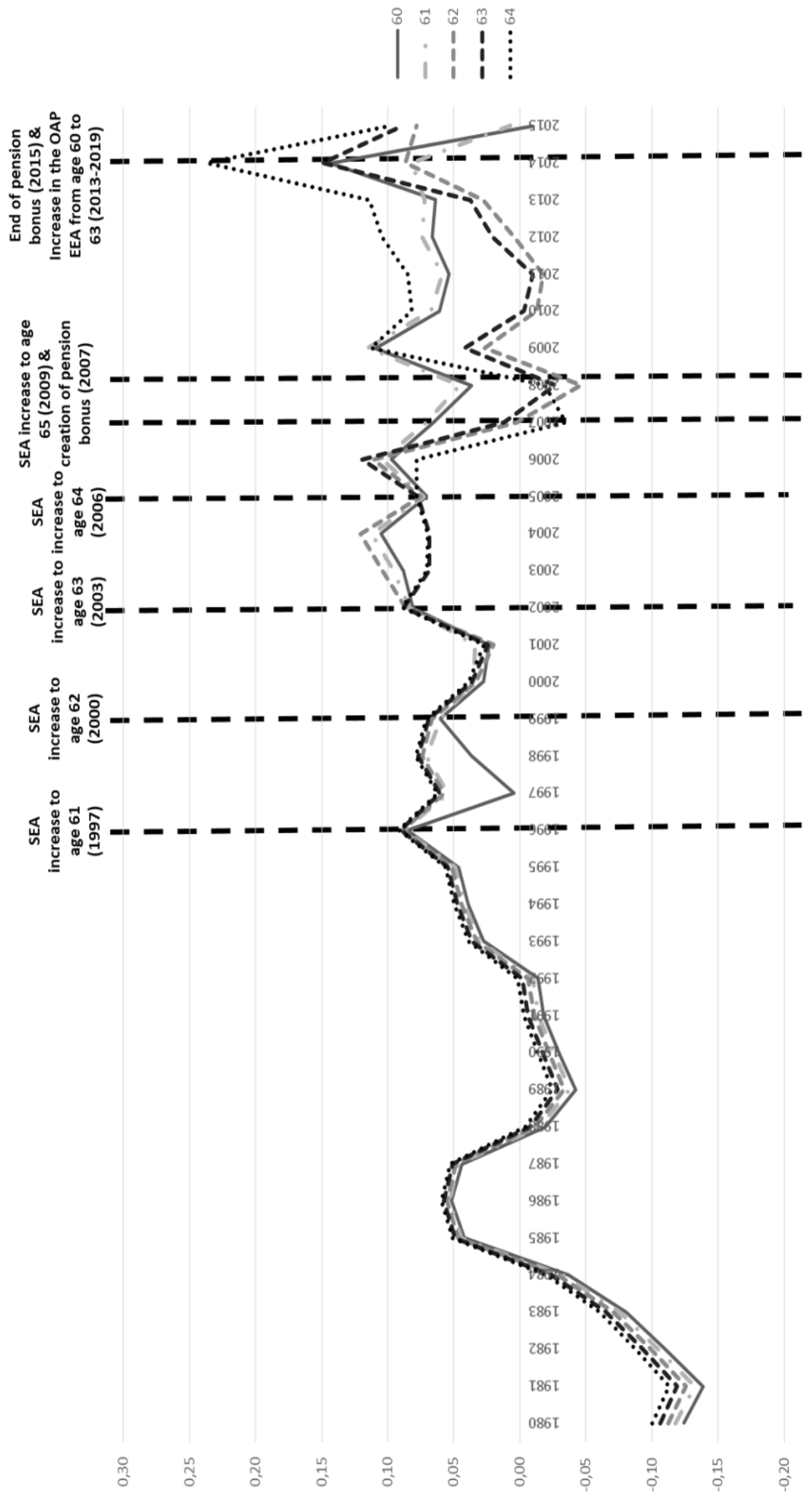

Source: Authors' own calculations 
When aggregating across income levels and focusing on changes in retirement incentives over time, very distinct patterns can be observed. Figure 3.22 and 3.23 present the implicit tax for four reference years, separated by 10 -year intervals each. While reforms during the first few decades have mostly focused on ages 55-59, there has been more recently a clear shift towards the age group 60-64. Results are, however, somewhat surprising: contrary to a general perception of an overall greater reward of longer working lives in the recent years, our results show that incentives are actually leaning heavily against longer working lives. Clearly, these results have to be read and interpreted with some caution: for example, the numerous special early retirement regimes that existed in addition to the headline conventional early retirement setup clearly affected the real-world incentives faced by individuals in ways that were sometimes quite different from the headline regime. Also, the weighting of the pathways remains somewhat contentious - with the residual weight on the old-age pension path likely overemphasizing its relevance. ${ }^{156}$

Figure 3.22: Implicit tax for male median earners by year

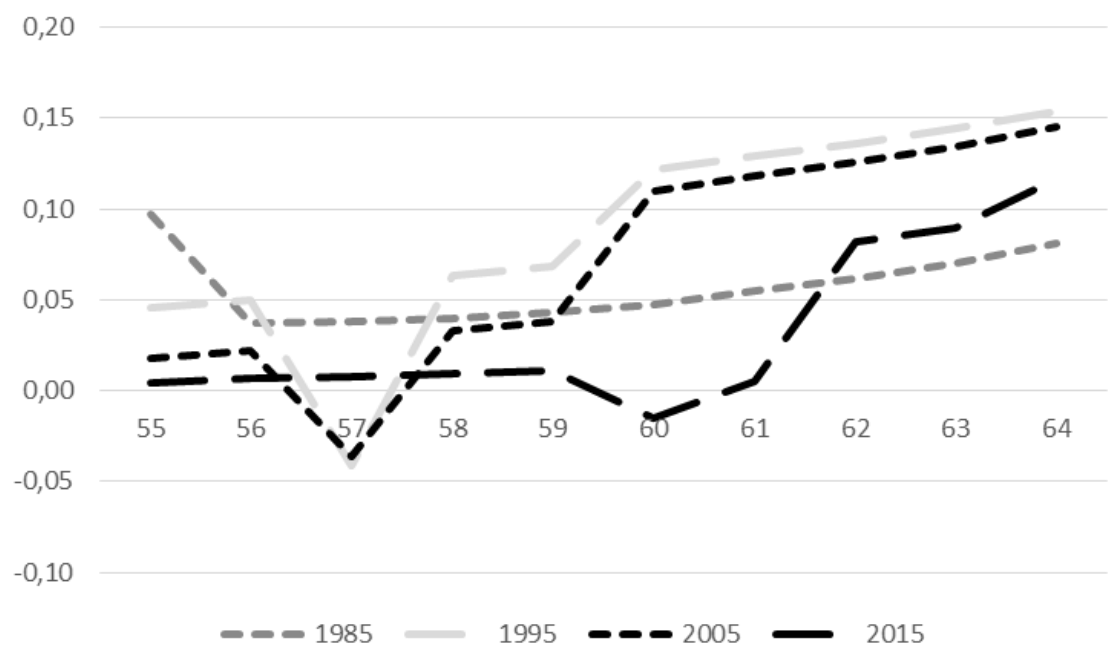

Source: Authors' own calculations

${ }^{156}$ Jousten and Lefebvre (2013) propose an alternative approach to determining the weights of the pathways. 
Figure 3.23: Implicit tax for female median earners by year

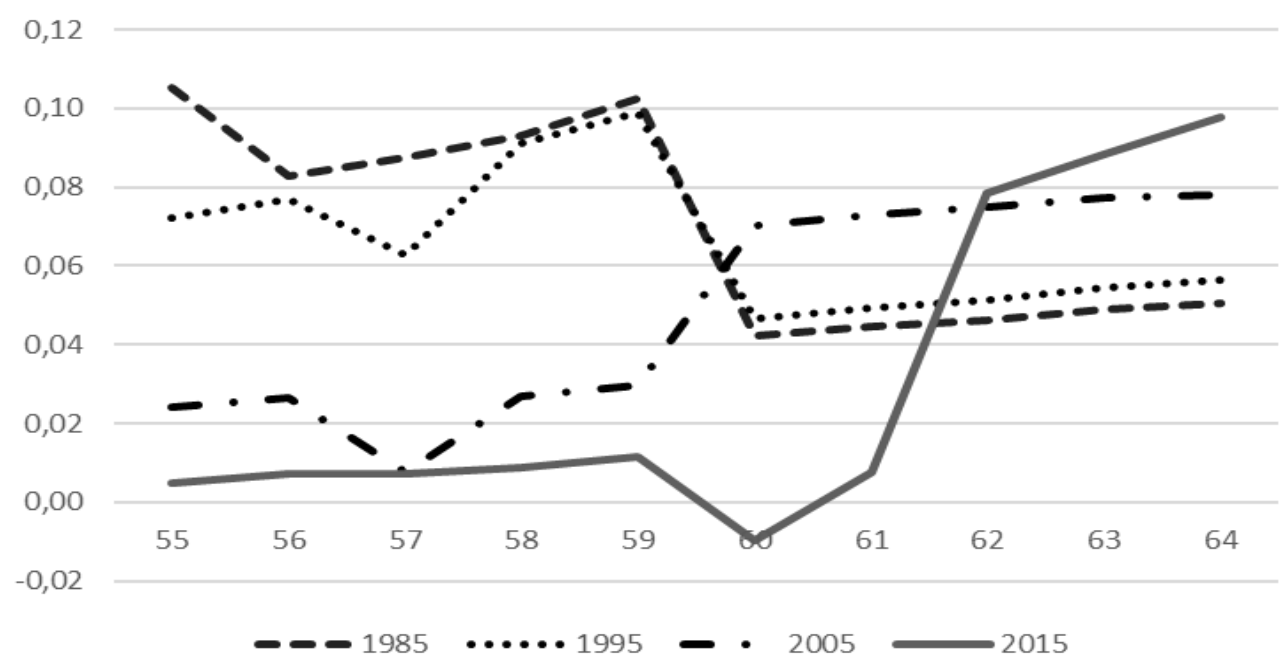

Source: Authors' own calculations

Similar caution should be applied when interpreting figures 3.24 to 3.27 . In these, we relate the average ITAX indicator by age groups of 55-59 and 60-64 in a given year to the employment rate of the same age groups in that year. Overall a negative relationship appears between the employment rate and the ITAX but not in a very strong manner. Clearly, the averaging across ages and the chosen weighting are likely disputable. However, we believe that even when correcting for some of these concerns (for example using a different weighting of exit routes that does not put the default weight on the oldage pension system) no stronger relation will emerge. This is likely due to the fact that individual incentives faced by real world Belgian workers are quite substantially different from those of the typical workers we have chosen. The real world is obviously less single, less complete career, with less stable earnings than our assumptions imply. On the other hand, it is also more diverse as employment, as defined by the labor force survey, also includes civil servants and self-employed. 
Figure 3.24: Employment rate and ITAX - men, ages 55-59

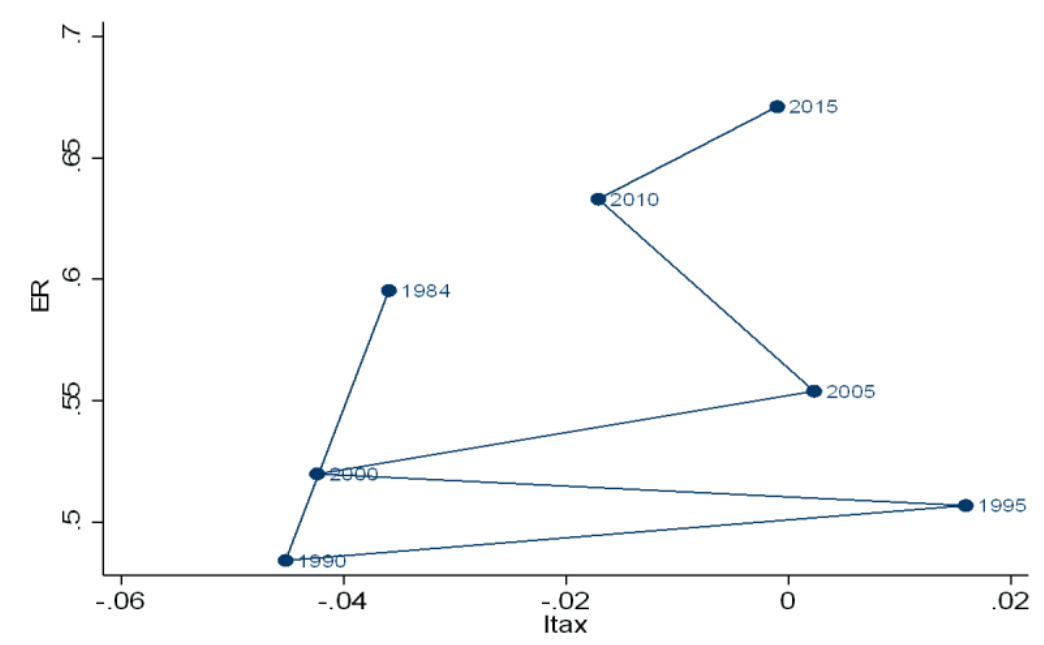

Source: Authors' calculations and Labor Force Survey

Figure 3.25: Employment rate and ITAX - women, ages 55-59

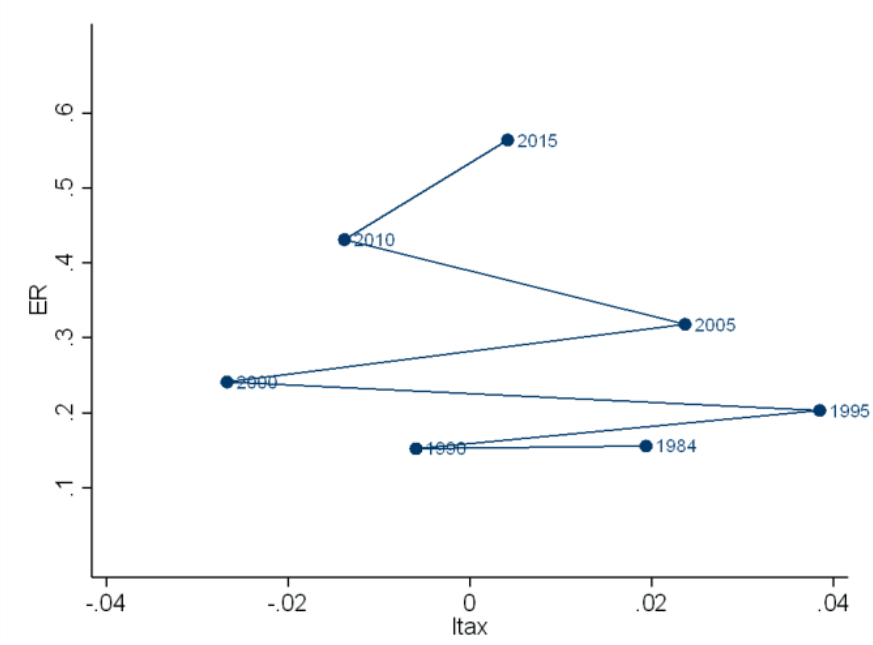

Source: Authors' own calculations and Labor Force Survey 
Figure 3.26: Employment rate and ITAX - men, ages 60-64

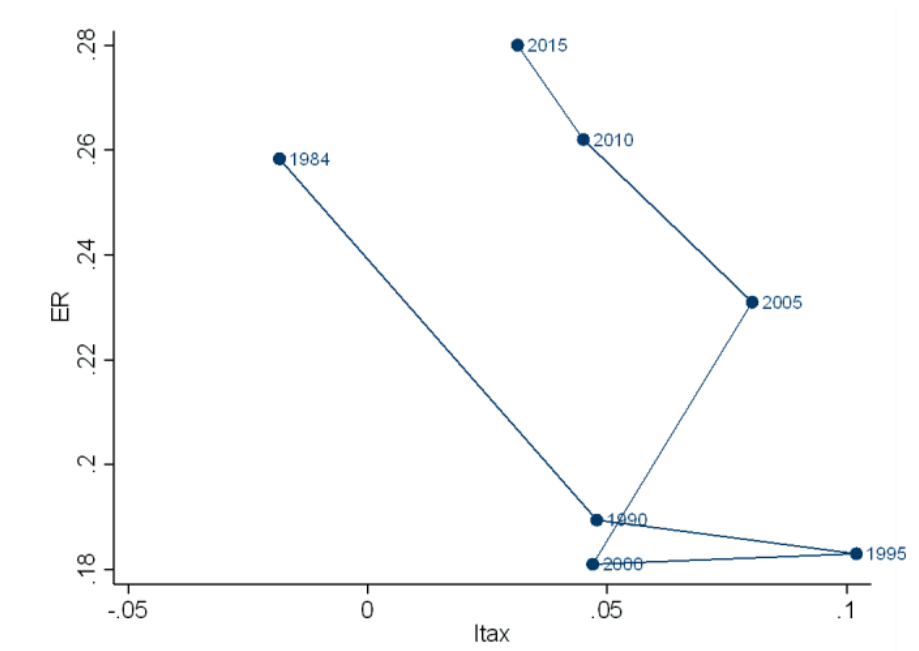

Source: Authors' own calculations and Labor Force Survey

Figure 3.27: Employment rate and ITAX - women, ages 60-64

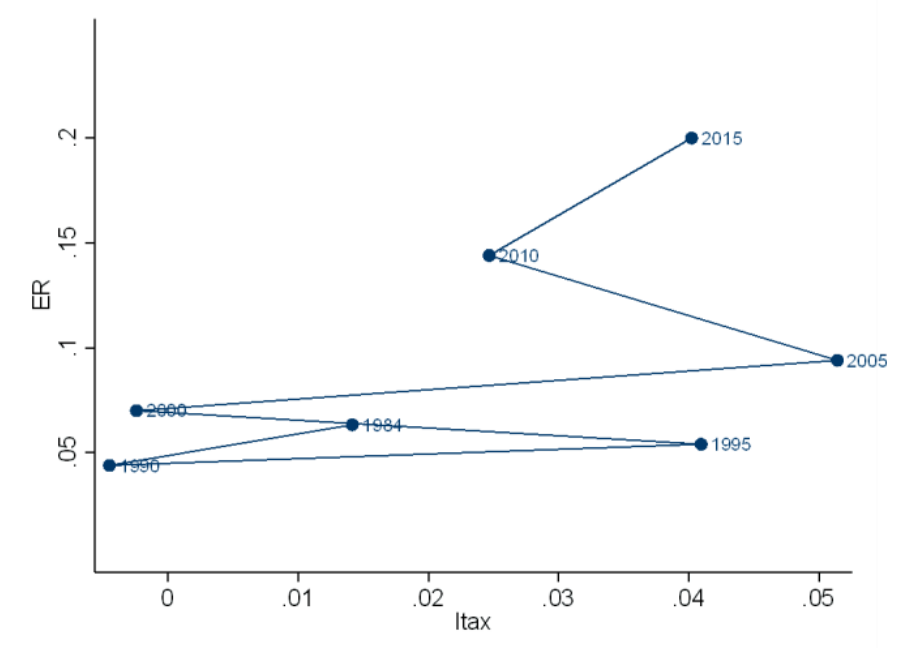

Source: Authors' own calculations and Labor Force Survey 


\subsection{RETIREMENT INCENTIVE: BELGIAN-SPECIFIC PROFILE}

In this section we present the incentive measures for the old-age pension for both sexes using the Belgian-specific scenario of earnings as described in Section 3.3. In addition to the median earner case, we also report the results of low and high earners since our assumptions on career length and earnings profiles may have important effects. For example, low income earners often qualify for the receipt of the minimum pension and some earnings of the median and high earners are replaced with the ceilings for pensionable income in the old-age pension benefit calculation.

Figure 3.28 presents the SSW for the old-age pension scheme by sex for the age group 6064 for the three selected earnings profiles. The results are somewhat different from what we obtained with the common profile. We will emphasize three main points that explain the differences with real-world relevance in the country.

First the Belgian-specific lifetime earnings profile assumes a continuous and almost constant increase of the wage throughout the career while the common profile assumes an important increase of the wage in the first years of career. Since old-age pension benefit are calculated on the average wage over the entire career, in the Belgian-specific scenario many more years with lower wages are included. In figure 3.28, this translates into lower SSW for all typical workers as compared to the results of the previous section. On the other hand, the use of the differently shaped Belgian earnings profile does not impact the build-up of SSW for low earners as they systematically benefit from the minimum pension under both scenarios. 
Second, the Belgian-specific profile assumes shorter careers. In this scenario, each category of worker starts working at a different age: male low earners at age 21 , female low earners at age 27, male median earners at age 25, female median earners at age 31 , male high earners at age 30 and female high earners at age 36. Shorter careers are translated into lower lifetime SSW for every type of worker. This is also true for the low earners for whom the minimum pension is adjusted according to the length of career.

Also, because we have assumed shorter careers in the Belgian-specific scenario, workers less often fulfil the career requirements for early retirement even if they reach the early eligibility age. Most notably, the reform that increased the career conditions for early oldage pension claiming from 20 years in 1997 to 35 in 2005 now directly affects the SSW of low and median-earning women as well as high earners of both sexes. This reform translates into a decrease in SSW for those who do not reach the career requirements for early retirement because each year of potential benefits before the statutory eligibility age is replaced by a zero. ${ }^{157}$ Male low and median earners are not directly impacted by this reform as they have sufficiently long careers to retire at the early eligibility age. However, as a result of the general increase of the early eligibility age, and the increase in stringency of the long-career exception, low and median-earner men also lose access to old-age pension benefits at age 60 as of the year 2013. Hence, their SSW at age 60 starts decreasing

\footnotetext{
157 More specifically: at age 60, low-earner women have 33 years of career and cannot access early retirement at age 60 from 2004 onwards: eligibility is age 61 in 2004, and 62 from 2005 onwards. Similarly, median-earner women have a career of 29 years when they reach the age of 60 and do not meet the career requirements for early retirement from 2002 onwards. High-earner men have a career of 30 years once they reach the age of 60 and hence do not meet the career requirement for early retirement from 2003 onwards. Finally, high earners women count 24 years of career at age 60 do not meet the career requirement for early retirement from 2000 onwards. From 2004 onwards, male high-earners have to wait until age 63 to retire and women high earners do not have any access to early retirement from 2005 onwards.
} 
as of 2013 as a result of the increase in early eligibility age to age 60.5. Similarly, their SSW at age 61 starts decreasing in 2015 when the early eligibility age increases to age 61.5.

Third, the impact of the pension bonus reform on individuals at different earnings levels can easily be identified on these graphs. The effect is comparable in absolute terms across income levels whatever the earnings level as the pension bonus is a constant amount given for any number of additional days worked above age 62 or after more than 44 years of career, independently of earnings levels and unaffected by pension ceilings.

Finally, the Belgian-specific scenario allows for the taxation rules to vary over the years instead of keeping them constant using 2016 tax laws. The specific impact of taxes in explaining differences in SSW between the common and the Belgian-specific scenarios is only visible for high earners above the age of 60: given the relative brevity of assumed careers, no old-age pension pensioner actually has to pay the disability or the solidarity contributions on pensions or income tax; high-earner old-age pensioners merely start paying a small amount of taxes at older ages from the early 1990's onwards; male medianearner old-age pensioners start paying a small amount of taxes as of age 65 starting in 1996; changes in the tax deduction for social security benefits are also mostly visible for high earners only, such as the 1998 decrease of the tax deduction for conventional early retirement benefits but remain trivial compared to the impact of eligibility reforms. ${ }^{158}$ The most important change in taxation happened for taxes on personal income, which has become less progressive over the years. Smaller taxes for high earners have increased their wage, which is translated into decreasing ITAX over the years. Once again the impact of such reform is fairly limited and mostly indistinguishable next to the eligibility reforms hence confirming the general validity of results from the common scenario analysis.

158 See chapter two for more information on the taxation of social security benefits. 
Figure 3.28: SSW- Old-age pension - ages 55-59

Low earners men

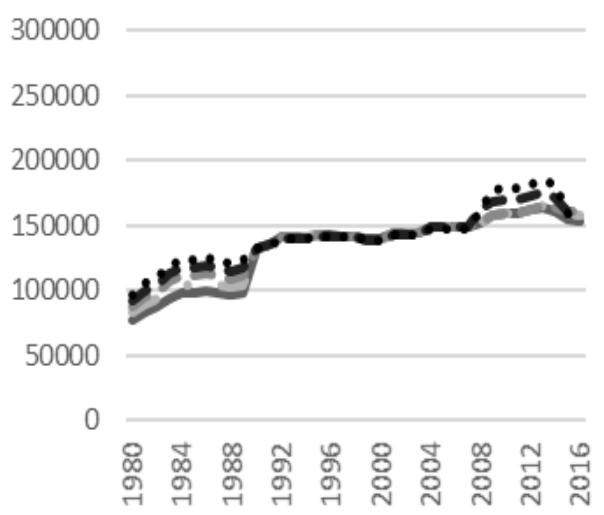

Median earners men

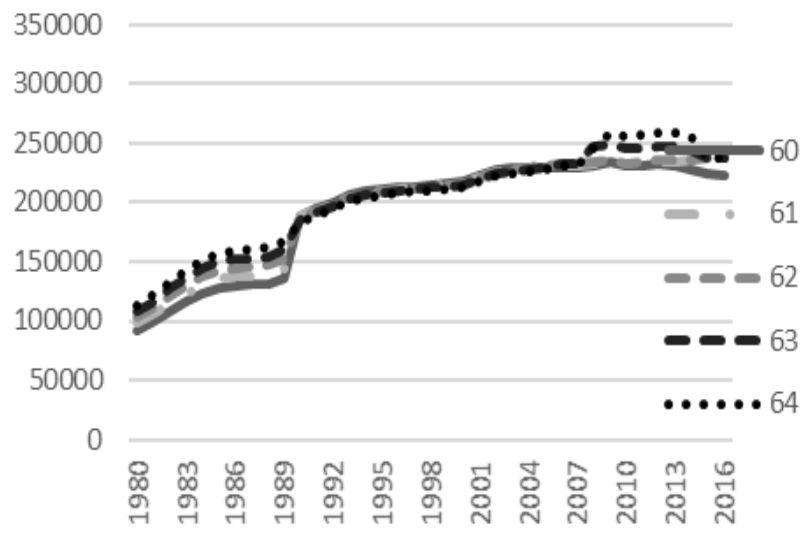

High earners men

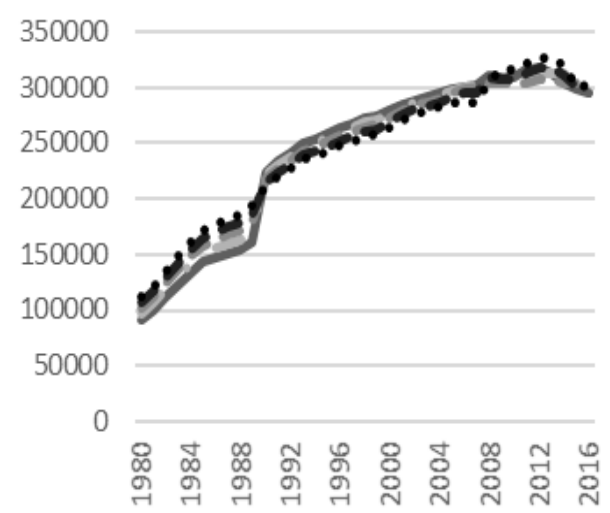

Low earners women

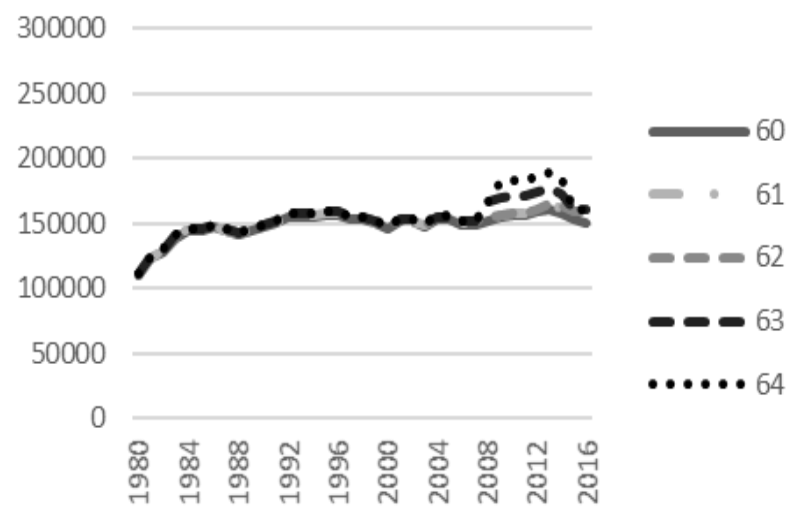

Median earners women

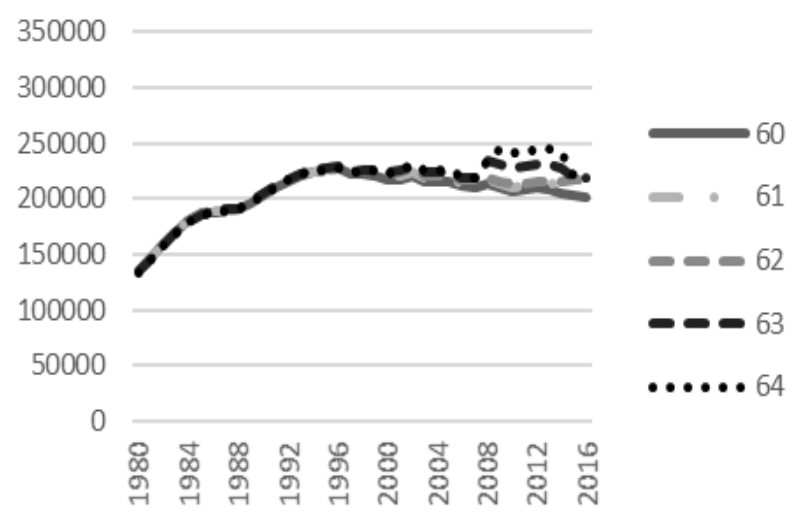

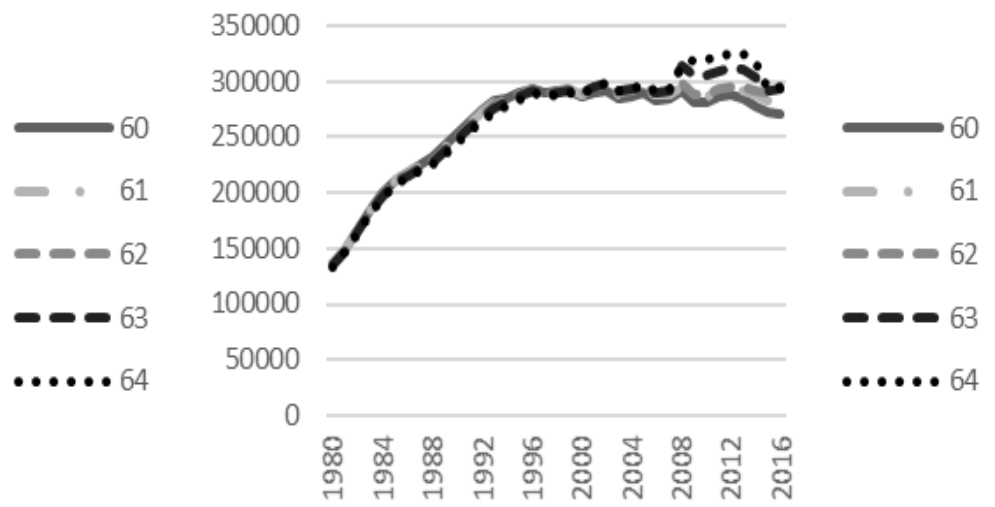

Source: Authors' own calculations 


\subsection{CONCLUSION}

The Belgian labor market has undergone major changes over the last decades. While the country was long characterized by low employment and labor force participation rates of the elderly male population, there was a reversal of this trend as the country witnessed a continuous increase in employment rates of older men from the early 2000's onwards. In this chapter, we explored the main institutional changes that have affected the retirement pathways of older workers from the 1980's until today. We simulated the retirement incentives faced by several typical workers, differentiated by age, sex and level of earnings, for each retirement year between 1980 and 2016, retirement ages ranging from 55 to 65 and for the 4 traditional labor force exit pathways. To do so, we used the concept of social security wealth and implicit tax on continued activity and we relied on two specific scenarios: a common one and a Belgian-specific one.

The results show that the various reforms have affected the incentive to retire through one or another pathway at the micro-level. We found that the tightening of eligibility conditions and the greater variation in the generosity of some benefits translate into important changes of our incentive measures to retire. These in turns appeared to be correlated, although marginally, with variations in the employment rates at the macrolevel.

However, these results are far from being clear-cut and the Belgian institutional landscape is likely to be more complex than the stylized view of the headline retirement and early retirement schemes we analyzed in this chapter. These first results open avenues for further research, better taking into account individual heterogeneity and the related social 
security generosity at the individual level. This would however require better information on eligibility of special or derogatory schemes - a nontrivial task. The results also show that there is room for micro-econometric analysis exploring the large individual variation in earnings histories and incentives. 


\section{REFERENCES}

Dellis, A., R. Desmet, A. Jousten, and S. Perelman (2004). Micro- Modeling of Retirement in Belgium. In Social Security Programs and Retirement around the World: MicroEstimation, edited by J. Gruber and D. Wise, 41-98. Chicago: University of Chicago Press.

Eurostat, (2017). European Labor Force Survey, European Union, Luxembourg.

Human Mortality Database (2017). University of California, Berkeley (USA), and Max Planck Institute for Demographic Research (Germany). www.humanmortality.de.

INAMI-RIZIV (various years). Statistiques des indemnités, INAMI-RIZIV: Brussels.

Jousten A., M. Lefebvre and S. Perelman (2012). Disability in Belgium. There is More than Meets the Eye, in D. Wise, Social Security and Retirement around the World : Historical Trends in Mortality and Health, Employment, and Disability Insurance Participation , University of Chicago Press and NBER.

Jousten, A., Lefebvre, M. and S. Perelman (2016). Health status, disability and retirement incentives in Belgium, in D. Wise, Social Security and Retirement around the World: Disability Insurance Programs and Retirement, 179-209. Chicago: University of Chicago Press and NBER.

Jousten A. and M. Lefebvre (2013). Retirement Incentives in Belgium: Estimations and Simulations Using SHARE Data, De Economist, vol. 161(3), pages 253-276

Jousten, A. and M. Lefebvre (2016). Older men's labor force participation in Belgium, eWorking Paper University of Liège, forthcoming in D. Wise, Social Security and 
Retirement around the World: men's labor force participation, University of Chicago Press and NBER.

Jousten A. and M. Lefebvre (2017). Spousal and survivor benefits in option value model of retirement: an application to Belgium. Journal of Pension Economics and Finance

ONEM-RVA (various years). Bénéficiaires de l'ONEM. ONEM-RVA : Brussels.

ONP-RVP (2005). Etudes Statistiques. ONP-RVP : Brussels.

ONP-RVP (various years). Statistiques annuelles des bénéficiaires de prestations. ONPRVP: Brussels.

Pestieau P. and J.P Stijns (1999).Social Security and Retirement in Belgium, in Social Security and Retirement around the World, edited by J. Gruber and D. Wise, National Bureau of Economic Research. 


\section{CHAPTER FOUR}

\section{TRENDS IN SOCIAL SECURITY INCENTIVES IN BELGIUM}

Anne-Lore Fraikin, Alain Jousten, Mathieu Lefebvre 


\subsection{INTRODUCTION}

Like many other European countries, Belgium has been exposed to a major transformation of its labor market environment over the last decades. At the same time, a large array of social security reforms has been implemented with the overarching goal of increasing the labor force participation through better work incentives - with a special focus on older workers. This chapter aims at assessing the role of those incentive-based reforms as an explanatory factor for the observed changes in older workers' employment patterns.

Previous studies for Belgium have already pointed at the role of social security incentives and institutional changes in explaining the labor force participation of the elderly (see i.e. Pestieau and Stijns, 1999; Dellis et al, 2004; Jousten and Lefebvre, 2013; 2019). However, they have generally covered much shorter time-spans and been unable to separate age effects from those of reforms.

In the third chapter of the thesis, we propose a first analysis over a more extended period. We use a typical-worker simulation approach akin to Pestieau and Stijns (1999) to assess the role of social security incentives on employment outcomes over the last four decades. We suggest that policy reforms in Belgium over the recent decades have focused more on implementation measures rather than on changes in key policy parameters. Relying on these typical worker simulations, we document a rather marginal effect of reforms on employment outcomes at the macro-level. However, these results cannot be considered as definitive, as a typical-worker approach is unable to reflect the institutional complexity - and the associated retirement incentive heterogeneity - of a country like Belgium with its highly segmented institutional landscape. Only micro-simulation and microestimation analysis can capture the prevailing degree of heterogeneity. 
In this chapter we provide a contribution to fill this gap. We investigate how individual social security incentives and particularly their changes over time explain the retirement decision at the micro level. We focus on the population of wage-earners ${ }^{159}$. For a sample of Belgian wage-earners aged 55-64 and for a period going from 2004 to 2010, we systematically calculate indicators of benefit entitlement and financial retirement incentive measures and we relate them to the individual labor force participation. We estimate a series of econometric specifications and use the results to simulate counterfactual labor supplies under a reform scenario.

The structure is as follows. Section 4.2 presents our dataset as well as the microsimulation approach we use to calculate financial retirement incentive measures. In Section 4.3, we show the results of a series of regressions using these financial incentive measures. Section 4.4 provides the counterfactual simulation analysis to scope the effect of social security reforms. Section 4.5 concludes.

\subsection{INCENTIVE MEASURES CALCULATION}

Based on the institutional setup described in chapter two, we compute several individuallevel financial incentive measures that summarize the generosity of the different labor force exit pathways: the old-age pension, the conventional early retirement, the unemployment insurance and the disability insurance programs. Below, we first present the data and then detail the formulae used to calculate financial incentives for each individual.

159 See chapter three for a more through explanation of why we choose to focus on wage-earners. 


\subsubsection{DATA}

The analysis relies on a representative administrative panel dataset drawn in 2004 and coming from the "Datawarehouse Labor Market and Social Protection". The data were provided by the Belgian Crossroad Bank for Social Security (CBSS) ${ }^{160}$. Information on individuals' labor market status are retrieved from the administrative records of various social security institutions and personal characteristics are retrieved from the national registry. The panel includes information on a quarterly basis for cohorts born between 1941 and 1949 for the period 2004 to 2010. For the purpose of this study, we use the calendar year as a period of reference, and hence merge the quarterly income data into a single yearly observation, using that status of the last day of the last quarter of each year.

We use a subsample of the original population that satisfies the double condition of working and of belonging to the wage-earner scheme in 2004, the start of the observation period. ${ }^{161}$ Although we focus our attention on the population of wage-earners, our sample inevitably includes people who currently have mixed employment histories as wage earners, civil-servants or self-employed. Also, current pure wage-earners may have had earlier spells as civil-servants or self-employed. For the purpose of our study we select an initial sample of individuals aged between 55 and 64 who were in active wage-earner employment in 2004 (part-time or full-time). ${ }^{162}$ Moreover, in each of the following sample years 2005-2009, we add individuals aged 55 in the given sample year, who satisfy the

\footnotetext{
${ }^{160}$ This dataset was initially created for the EMPOV project (Employment and Poverty in a Changing Society), financed by the Belgian Science Policy Administration (BELSPO research project TA/00/45). ${ }^{161}$ A similar condition is applied to spouses in each year of analysis.

162 The workers who are still actively employed but receive some forms of (retirement-related) social security benefit are included. Coincidentally, the sample includes no persons aged 64 in 2004.
} 
double condition of having been in active wage-earner employment in 2004 and still being in this status. We follow each individual until 2010, age 65 or until retirement. ${ }^{163}$

We obtain a sample of 86,666 observations with possible retirement ages ranging from 56 to 65 in years from 2005 to 2010, which corresponds to 26,880 distinct individuals. Table 4.1 presents descriptive statistics of the 26,880 individuals, as they appear in their first observation year. Based on the active employment condition at older ages that we impose, we obtain a sample with a stronger male component than for the Belgian population as a whole. In line with the wider population, women in our sample have shorter careers than men. ${ }^{164}$ The breakdown of the sample by region implies that older Flemish men are more frequently still at work than in other regions. The majority of individuals are married.

Table 4.1: Main Characteristics of the individuals present in the sample, in their first

\begin{tabular}{cccc}
\multicolumn{3}{c}{ observation year } \\
\hline All & Men & Women \\
\hline$N$ & & & \\
\hline Breakdown by Region & 26,880 & 16,981 & 9,899 \\
Flanders & & & \\
Wallonia & $61.7 \%$ & $63.9 \%$ & $58 \%$ \\
Brussels & $29.6 \%$ & $28.5 \%$ & $31.4 \%$ \\
Married & $8.7 \%$ & $7.6 \%$ & $10.6 \%$ \\
Breakdown by Marital Status & & & \\
Unmarried or single & $72.8 \%$ & $77.1 \%$ & $62.7 \%$ \\
Average years of career & $28.2 \%$ & $22.9 \%$ & $37.3 \%$ \\
& 33.3 & 34.6 & 31.1 \\
\hline
\end{tabular}

Note: Author's calculations based on CBSS data.

\footnotetext{
${ }^{163}$ Individuals who die or transfer to civil servant or self-employed status are also withdrawn from the sample.

164 Because of the activity condition at older ages that we imposed, the sample of women we have selected have relatively longer working careers than in the total population of Belgian women at older ages.
} 
Figures 4.4 and 4.5 present the observed exit rates by age for years 2005-2010 at possible retirement ages 56 to 65 . These exit rates correspond to the transition from paid employment to inactivity through one of the four main exit pathways (residual exits ${ }^{165}$ are included in the old-age pension pathway). Some key ages are important: the ages of 58 (the early eligibility age for conventional early retirement), 60 (old-age pension early eligibility age and conventional early retirement eligibility age) and 65 (old-age pension statutory eligibility age) are modal labor market exit ages. For women the situation is slightly different at the upper end of the age spectrum as the statutory eligibility age of the old-age pension increased during our sample period from 63 to 65 (reform of 1997) hence limiting the pivotal role of age 65 .

Figure 4.4: Exit rates by age for men - all sample

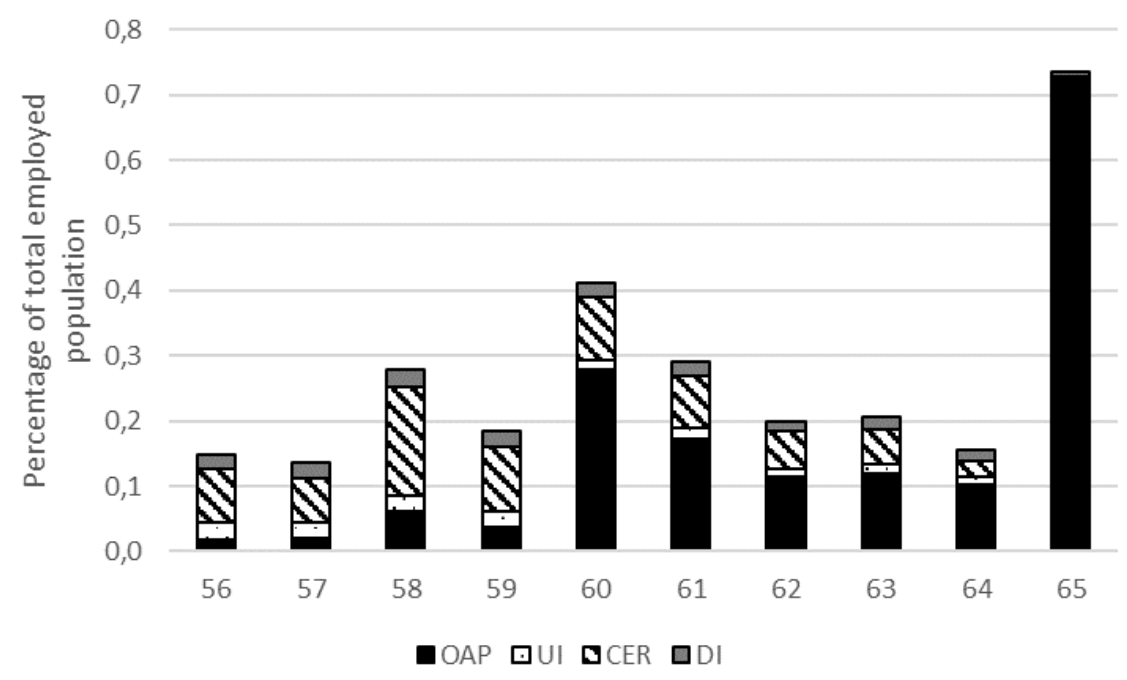

Note: Full sample of person-year observations 2005-2010. Author's calculations based on CBSS data.

165 These includes exits through social aid, professional diseases, work injury, etc. 
Figure 4.5: Exit rates by age for women - all sample

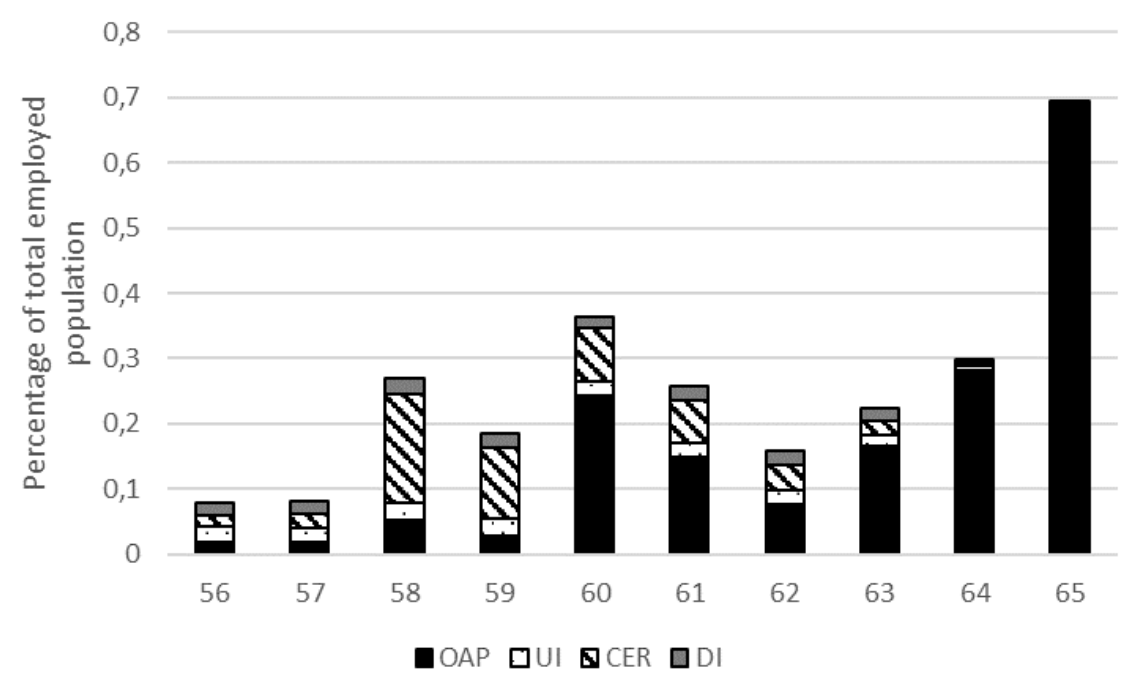

Note: Full sample of person-year observations 2005-2010. Author's calculations based on CBSS data.

\subsubsection{BENEFITS CALCULATION}

For each individual in the sample and for each year of observation, we compute the aftertax stream of benefits that he or she could claim in case of exit through each of the four exit routes applying the rules at this possible retirement age- also using tax rules as applicable in the possible year of retirement. Expressed differently, for each individual $i$ of age $a$, we calculate the after-tax benefit $B_{k, a}(R, i)$ from the exit pathway $k$ in case of retirement at age $R$, where $R \in[56,65]$. Benefits are equal to the benefit entitlement from exit pathway $k$ for all ages $a \geq R$.

For exits through the unemployment and disability programs, no early entitlement ages exist. For conventional early retirement exits, we do not verify the eligibility conditions as there are many exceptions to these rules that we cannot validate with our data. Hence positive benefits are applied for all $a \geq R$ in the unemployment, disability and conventional early retirement exit pathways with automatic rollover into old-age pension at the 
statutory eligibility age. For old-age pension exits, given strict eligibility conditions, zero benefits are imputed in periods $t$ where the eligibility conditions are not yet met, applicable positive benefits are counted otherwise. Once positive benefits are claimed, we assume they remain constant in real terms in future years as long as benefits are payable.

We apply the social security rules for eligibility and benefit calculations as they were applicable for each year of first entitlement based on each individual's career history, marital status and occupation as well as the partner's occupation. Because social security and taxation rules differ greatly according to the spouse's occupational status, we combine information from the national registry and individual occupational status to identify one-earner and two-earner couples in order to calculate the social security benefits accordingly.

To validate our old-age pension benefit simulator, we compute old-age pension benefit entitlements using our simulation tool and we compare them with the observed realworld old-age pension entitlements that individuals in our sample, who retired before the end of our observation period, are granted as indicated in the pension administration administrative records. As shown in Figure 4.6, both distributions are very close. For individuals who belong to our sample and who retired between 2005 and 2010, we find an average simulated pension of 1417.7 euros and an average real pension of 1388.1 ( euros the first year of retirement). ${ }^{166}$ In total, $87.6 \%$ of our simulated pensions are less than 200 euros from the corresponding observed real pension amount.

\footnotetext{
166 The remaining differences between our simulations and the real pensions are plausibly caused by missing data on personal characteristics and special career histories. For instance, we lack information on years on self-employment before 2004, those years are used in the accessibility criteria for and calculation of the minimum pension, which leads to an underestimation of the amount of minimum pension received by some individuals.
} 
Figure 4.6: Comparison of observed and simulated old-age pension benefits, old-age pension retirees in the sample 2005-2010

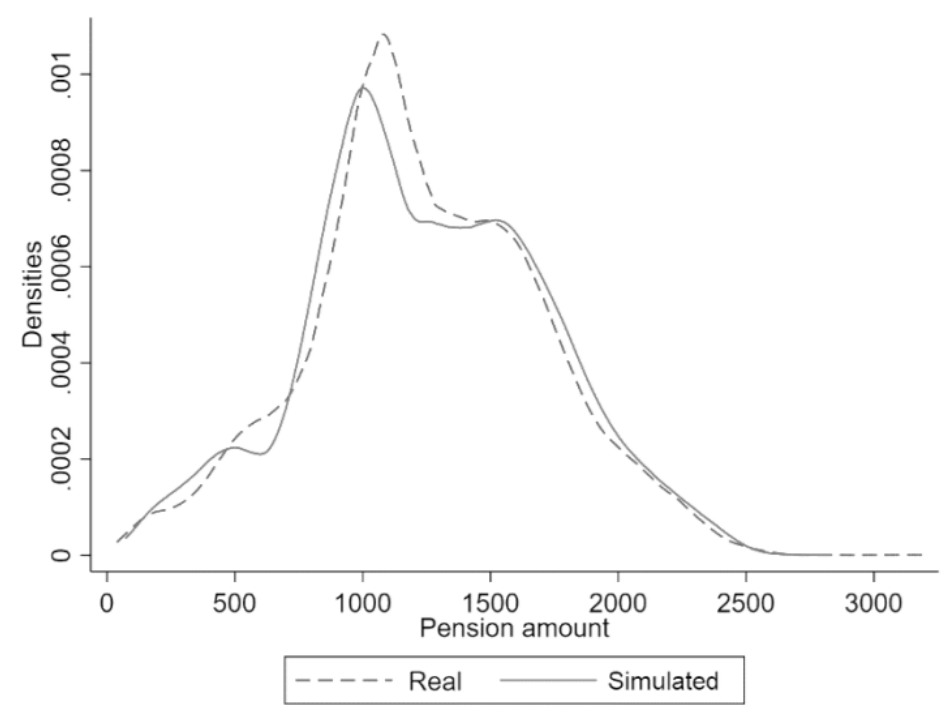

Note: Author's calculations based on CBSS data.

\subsubsection{INCENTIVE MEASURES}

From these annual benefit amounts by scheme, we calculate three aggregate indicators of incentives for each individual: the aggregate social security wealth (SSW), the aggregate accrual of SSW (ACC) and the implicit tax on continued activity (ITAX). ${ }^{167}$

Figure 4.7 presents the average SSW for each exit pathway by age for every year of observation and separately for men and women. The conventional early retirement pathway is the most generous scheme in terms of benefits for men and women. The disability presents the second highest average benefits thanks to relatively generous benefits ceilings (at least as compared to the unemployment and conventional early retirement). The third most favorable exit pathway is the unemployment, which includes the seniority supplement from age 58 onwards, but has relatively low benefits ceilings.

\footnotetext{
${ }^{167}$ See Chapter three for a description of the social security wealth, the accrual and the implicit tax on continued activity.
} 
The receipt of these benefits is combined with continued pension accruals until the statutory eligibility age. The least favorable exit pathway is the old-age pension scheme that is only available from age 60 onwards. Thus, the SSW of the old-age pension exit pathway increases with age before 60 because we assume that individuals receive an income of zero until they become eligible for the old-age pension. From age 60 until the statutory eligibility age, individuals can access the old-age pension if they meet the minimum career requirement (34 years in 2004 and 35 years from 2005 until 2013) and the SSW decreases as age increases. This reversal in average SSW amounts is caused by the fact that delaying claiming now has an instantaneous cost in terms of benefits payable the latter cost being effectively larger than the gain from adding one year of earnings to the old-age pension benefits calculation. At age 65 , it is not possible to receive unemployment, conventional early retirement or disability benefits anymore and workers are automatically transferred to the old-age pension system. Thus, the difference in SSW between the old-age pension and the other exit pathways decreases as we get closer to age 65 because there are fewer years spent in the unemployment, conventional early retirement or disability schemes. 
Figure 4.7: Average SSW by exit pathway (in 2010 euros)
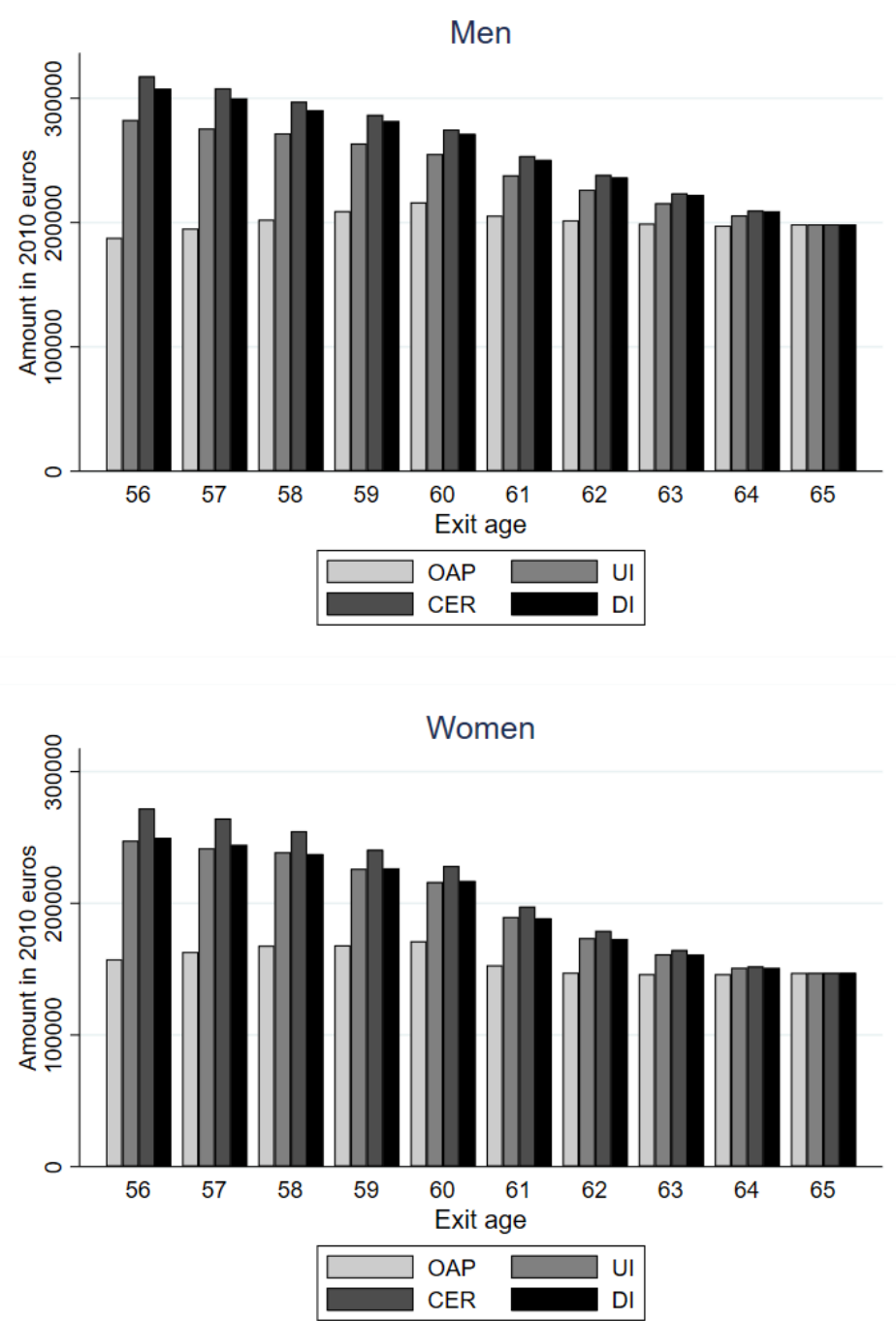

Note: Full sample of person-year observations 2005-2010. Author's calculations based on CBSS data.

We then aggregate these scheme-specific SSW measures into one aggregated SSW indicator, which sums the previously scheme-specific incentive measures. We use an aggregation method that is similar to the one applied by Dellis et al (2004): For each pathway except old-age pension, we use the number of observed exits into each route from a certain age until the statutory eligibility age as a proportion of the total number of 
employed individuals at each age as weights, and we further differentiate the weights by sex and year. ${ }^{168}$ The old-age pension scheme takes the residual weight.

Figure 4.8 presents the aggregated SSW measure for men and women separately. The incentives are mostly driven by the SSW of the old-age pension and of the conventional early retirement (from age 56 to 60 only). The aggregated SSW of men and women increases at age 58 following an increase in the conventional early retirement participation rate, and then decreases until age 64 because of the growing influence of the SSW of the old-age pension. Unsurprisingly, the SSW is lower for women than for men because of lower average pension entitlements.

168 See appendix for the weights used in the aggregation method. 
Figure 4.8: Average aggregated individual SSW, by gender (in 2010 euros)
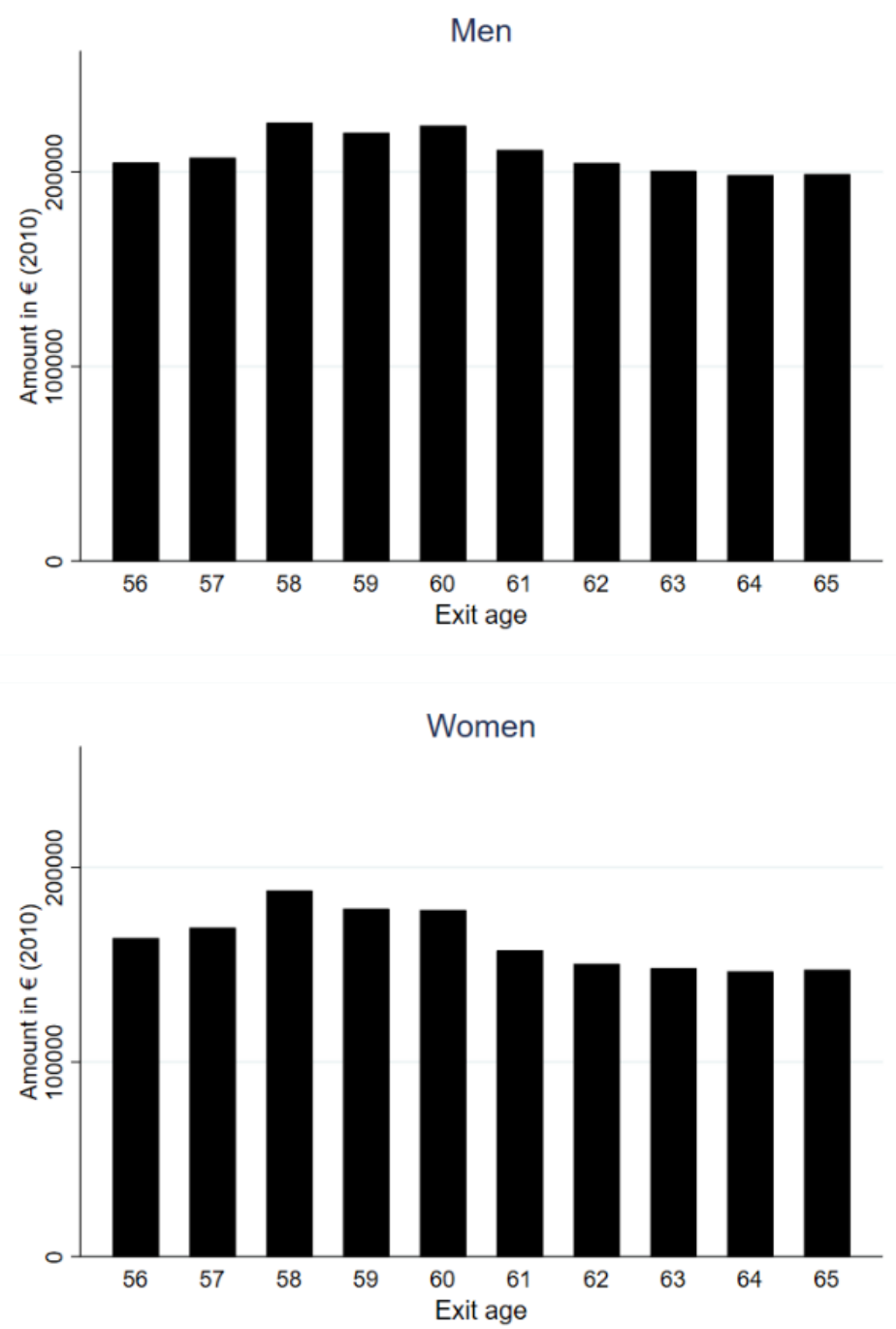

Note: Full sample of person-year observations 2005-2010. Author's calculations based on CBSS data.

Figure 4.9 and 4.10 illustrate the aggregated ACC and ITAX measures for both sexes. ${ }^{169}$ The ITAX of men and women is negative for exits between 56 and 59. This is mainly due to the influence of the old-age pension system, which is not available at those ages. Individuals are imputed a pension of zero until the old-age pension system becomes available at the early eligibility age of 60 if they have a career that is long enough or at the

${ }^{169}$ See Chapter three for a description of the accrual and the implicit tax on continued activity. 
statutory eligibility age. The aggregated ITAX of men is less negative than that of women because of the larger influence of the conventional early retirement program, associated with large positive ITAX. From age 60 onwards, the ITAX turns positive for men as they have largely reached the old-age pension early eligibility age. As for women, since they are less likely to access the early eligibility age old-age pension because of career conditions, the ITAX generated by the old-age pension remains negative for the same reasons mentioned above. At age 65, the ITAX is only composed of benefits from the OAP pathway since other pathways are not available anymore.

Figure 4.9: Average ACC, by gender (in 2010 euros)
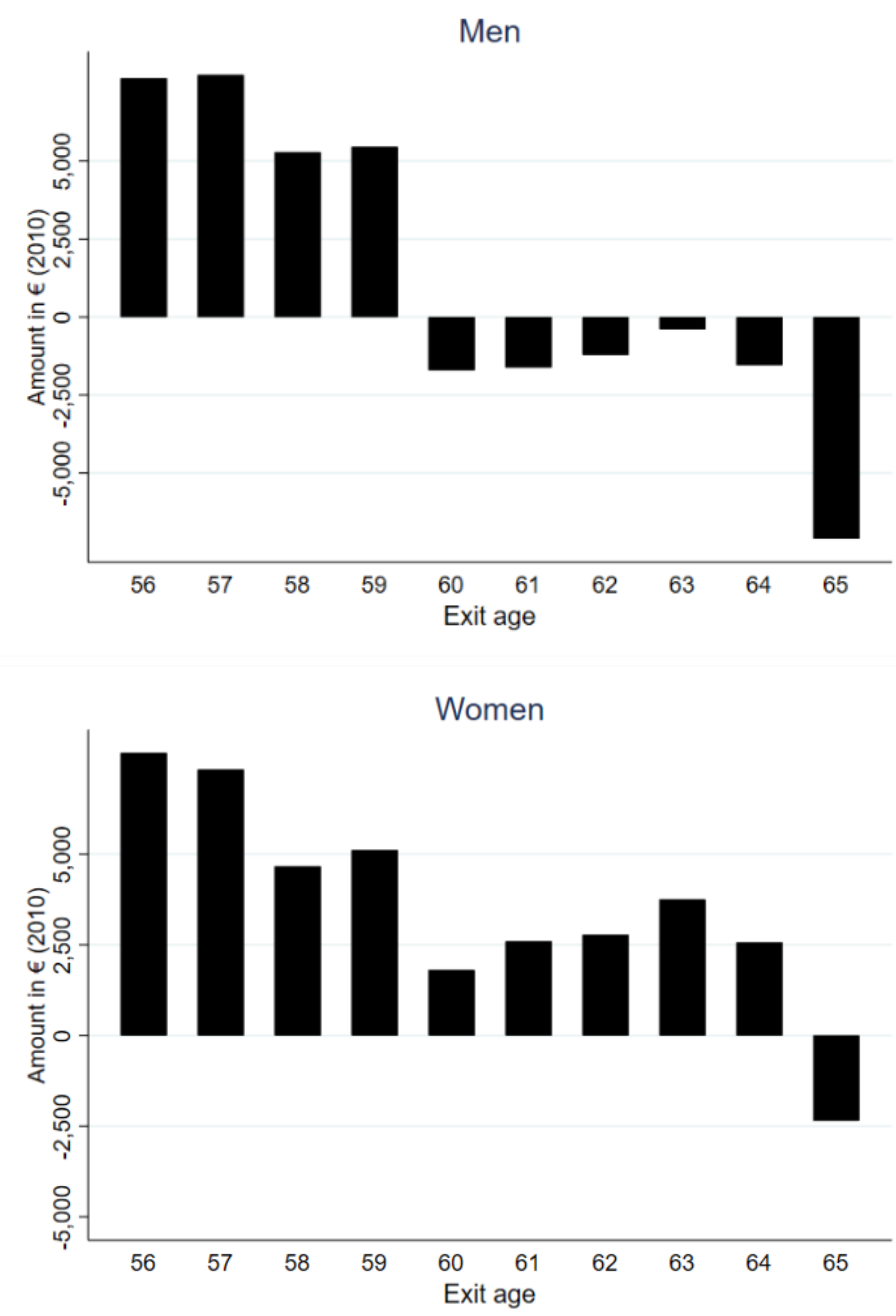

Note: Full sample of person-year observations 2005-2010. Author's calculations based on CBSS data. 
Figure 4.10: Average ITAX, by gender (in 2010 euros)
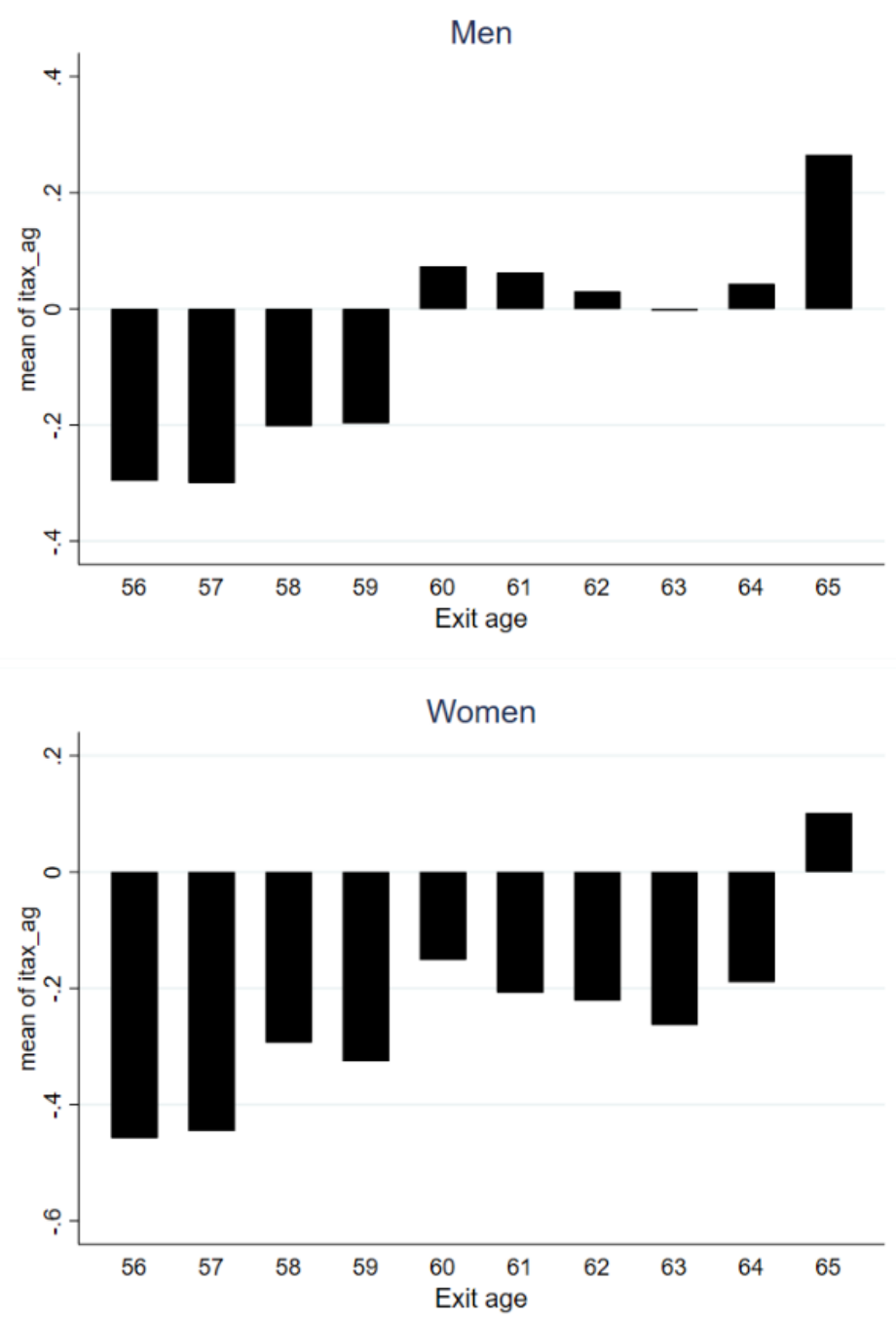

Note: Full sample of person-year observations 2005-2010. Author's calculations based on CBSS data. 


\subsection{REGRESSION RESULTS}

In this section we present the regression results we obtained from five different models: a standard linear probability model, the same model with fixed effects and random effects and a probit model with and without random effects.

The dependent variable is retirement; it is equal to one if the individual exits employment through one of the four exit pathways within the year, and zero otherwise. It corresponds to the observed exit patterns presented in figures 4.4 and 4.5 .

We use the previously derived three incentive measures as explanatory variables, and control for a large set of personal and socio-demographic variables: being a female, having a partner, having an active partner, a dummy if the individual lives in Wallonia or Brussels (living in Flanders is the reference) and age (and its squared form). We control for the individual's earnings and of his or her partner by including indicators of average lifetime earnings ${ }^{170}$ and the current salary (and their squared forms).

We control for job related characteristics: part-time work, white-collar worker (with bluecollar worker as reference) and two binary variables indicating if the individual works in the primary or the tertiary industry sectors (working in the secondary sector is the reference). Finally, we add an eligibility binary variable that indicates whether the individual has reached the statutory eligibility age of old-age pension, set at age 65 for men and 63, 64, 65 for women between 2003 and 2005, 2006 and 2008 and after 2009,

\footnotetext{
170 The average lifetime earnings for the reference person is calculated as $\frac{\text { lifetime earnings }}{(\text { age-16) }}$ and the average lifetime earnings for the partner is calculated as $\frac{\text { lifetime earnings }}{45}$ because of a lack of data on the partner's age (45 is the standard complete career).
} 
respectively. ${ }^{171}$ For the probit regressions, we report marginal effects estimated at the mean.

Table 4.2 presents the results of our five models using the total sample of individuals aged 56 to 65 between the year 2005 to $2010 .{ }^{172}$ We report the results for models using the SSW and the ITAX measures of incentive in the body of the text. ${ }^{173}$ The marginal effect of ITAX is positive and significant in every model, which means that a larger tax on continued activity leads to higher probability of retirement. In all models except for the fixed effect model, the effect of SSW is positive and significant. The fixed effect model removes the impact of invariant unobserved individual characteristics such as demand for leisure and time preferences. It is likely that the SSW measure in the other models is positively correlated with these unobserved variables and thus captures their positive effect on the retirement probability. We are thus left with a negative and significant impact of the SSW on the retirement probability in the fixed effects model - a result that is in line with previous findings for Belgium (Dellis et al, 2004). The fixed effects results should, however, be read with caution as we only have a limited number of periods of observation for each person in our sample (3.33 on average) and some unobserved characteristics (health trends, e.g.) might also be age-varying.

\footnotetext{
${ }^{171}$ While age dummies are potentially well equipped to capture age-based focal points and bounded rationality concerns as in the behavioral economics literature on the subject (Seibold, 2017), eligibility variables also have their role to play as they capture factors going beyond age and financial incentives. This includes, for example, issues of liquidity constraints. In chapter six, we explore the effect of different age specifications in details.

${ }^{172}$ Because of the dynamic setting of our dependent variable, we do not include the first observation year of individuals, as the dependent variable cannot vary at age 55 or in year 2004.

173 The regressions that include the accrual measure as explanatory variable instead of the ITAX measure present very similar results and are presented in the appendix.
} 
Table 4.2: Retirement - Total sample

\begin{tabular}{|c|c|c|c|c|c|}
\hline & LPM & Fixed effect & $\begin{array}{c}\text { Random } \\
\text { effect }\end{array}$ & Probit & $\begin{array}{l}\text { Probit } \\
\text { random } \\
\text { effects }\end{array}$ \\
\hline ITAX & $\begin{array}{c}0.079^{* * *} \\
(0.005)\end{array}$ & $\begin{array}{c}0.148^{* * *} \\
(0.007)\end{array}$ & $\begin{array}{c}0.093^{* * *} \\
(0.005)\end{array}$ & $\begin{array}{c}0.063^{* * *} \\
(0.005)\end{array}$ & $\begin{array}{c}0.705^{* * *} \\
(0.006)\end{array}$ \\
\hline SSW/100,000 & $\begin{array}{c}0.162^{* * *} \\
(0.004)\end{array}$ & $\begin{array}{c}-0.178^{* * *} \\
(0.014)\end{array}$ & $\begin{array}{c}0.202^{* * *} \\
(0.006)\end{array}$ & $\begin{array}{c}0.172^{* * *} \\
(0.004)\end{array}$ & $\begin{array}{c}0.194^{* * *} \\
(0.005)\end{array}$ \\
\hline Female & $\begin{array}{c}-0.053^{* * *} \\
(0.003)\end{array}$ & & $\begin{array}{c}-0.069^{* * *} \\
(0.005)\end{array}$ & $\begin{array}{c}-0.055^{* * *} \\
(0.003)\end{array}$ & $\begin{array}{c}-0.063^{* * *} \\
(0.004)\end{array}$ \\
\hline Age & $\begin{array}{c}0.559^{* * *} \\
(0.029)\end{array}$ & $\begin{array}{c}0.461^{* * *} \\
(0.036)\end{array}$ & $\begin{array}{c}0.393^{* * *} \\
(0.029)\end{array}$ & $\begin{array}{c}0.610^{* * *} \\
(0.029)\end{array}$ & $\begin{array}{c}0.633^{* * *} \\
(0.030)\end{array}$ \\
\hline Age squared & $\begin{array}{c}-0.005^{* * *} \\
(0.000)\end{array}$ & $\begin{array}{c}-0.003^{* * *} \\
(0.000)\end{array}$ & $\begin{array}{c}-0.003^{* * *} \\
(0.000)\end{array}$ & $\begin{array}{c}-0.005^{* * *} \\
(0.000)\end{array}$ & $\begin{array}{c}-0.005^{* * *} \\
(0.000)\end{array}$ \\
\hline SEA & $\begin{array}{c}0.354^{* * *} \\
(0.010)\end{array}$ & $\begin{array}{c}0.408^{* * *} \\
(0.011)\end{array}$ & $\begin{array}{c}0.335^{* * *} \\
(0.010)\end{array}$ & $\begin{array}{c}0.381^{* * *} \\
(0.016)\end{array}$ & $\begin{array}{c}0.248^{* * *} \\
(0.009)\end{array}$ \\
\hline Partner & $\begin{array}{c}-0.012^{* * *} \\
(0.003)\end{array}$ & $\begin{array}{c}0.001 \\
(0.015)\end{array}$ & $\begin{array}{c}-0.015^{* * *} \\
(0.005)\end{array}$ & $\begin{array}{c}-0.018^{* * *} \\
(0.003)\end{array}$ & $\begin{array}{c}-0.021^{* * *} \\
(0.004)\end{array}$ \\
\hline Active partner & $\begin{array}{c}0.000 \\
(0.003)\end{array}$ & $\begin{array}{c}-0.017^{* * *} \\
(0.005)\end{array}$ & $\begin{array}{c}0.005 \\
(0.004)\end{array}$ & $\begin{array}{c}0.004 \\
(0.003)\end{array}$ & $\begin{array}{c}0.006^{*} \\
(0.003)\end{array}$ \\
\hline Brussels & $\begin{array}{c}-0.021^{* * *} \\
(0.004)\end{array}$ & & $\begin{array}{c}-0.036^{* * *} \\
(0.007)\end{array}$ & $\begin{array}{c}-0.020^{* * *} \\
(0.004)\end{array}$ & $\begin{array}{c}-0.024^{* * *} \\
(0.005)\end{array}$ \\
\hline Wallonia & $\begin{array}{c}-0.012^{* * *} \\
(0.003)\end{array}$ & & $\begin{array}{c}-0.017^{* * *} \\
(0.004)\end{array}$ & $\begin{array}{c}-0.011^{* * *} \\
(0.003)\end{array}$ & $\begin{array}{c}-0.013^{* * *} \\
(0.003)\end{array}$ \\
\hline Part-time & $\begin{array}{c}0.021^{* * *} \\
(0.003)\end{array}$ & $\begin{array}{c}-0.064^{* * *} \\
(0.006)\end{array}$ & $\begin{array}{c}0.004 \\
(0.004)\end{array}$ & $\begin{array}{c}0.022^{* * *} \\
(0.003)\end{array}$ & $\begin{array}{c}0.022^{* * *} \\
(0.003)\end{array}$ \\
\hline White collar & $\begin{array}{c}-0.036^{* * *} \\
(0.003)\end{array}$ & $\begin{array}{c}-0.082^{* * *} \\
(0.024)\end{array}$ & $\begin{array}{c}-0.053^{* * *} \\
(0.005)\end{array}$ & $\begin{array}{c}-0.035^{* * *} \\
(0.003)\end{array}$ & $\begin{array}{c}-0.039^{* * *} \\
(0.003)\end{array}$ \\
\hline Primary sector & $\begin{array}{c}-0.038^{* * *} \\
(0.014)\end{array}$ & & $\begin{array}{c}-0.059^{* * *} \\
(0.023)\end{array}$ & $\begin{array}{c}-0.036^{* * *} \\
(0.011)\end{array}$ & $\begin{array}{c}-0.046^{* * *} \\
(0.016)\end{array}$ \\
\hline Tertiary sector & $\begin{array}{c}0.003 \\
(0.002)\end{array}$ & & $\begin{array}{c}0.020^{* * *} \\
(0.004)\end{array}$ & $\begin{array}{c}0.001 \\
(0.002)\end{array}$ & $\begin{array}{c}0.002 \\
(0.003)\end{array}$ \\
\hline $\begin{array}{l}\text { Average lifetime } \\
\text { earnings/1000 }\end{array}$ & $-0.195^{* * *}$ & $-2.001^{* * *}$ & $-0.254^{* * *}$ & $-0.213^{* * *}$ & $-0.243^{* * *}$ \\
\hline Squared form & $\begin{array}{c}(0.010) \\
0.019^{* * *} \\
(0.002)\end{array}$ & $\begin{array}{c}(0.144) \\
0.261^{* * *} \\
(0.017)\end{array}$ & $\begin{array}{c}(0.014) \\
0.028^{* * *} \\
(0.003)\end{array}$ & $\begin{array}{c}(0.010) \\
0.019^{* * *} \\
(0.002)\end{array}$ & $\begin{array}{l}(0.012) \\
0.22^{* * *} \\
(0.002)\end{array}$ \\
\hline $\begin{array}{l}\text { Current } \\
\text { salary/1000 }\end{array}$ & $-0.033^{* * *}$ & $-0.088^{* * *}$ & $\begin{array}{c}-0.054^{* * *} \\
(0.004)\end{array}$ & $-0.028^{* * *}$ & $\begin{array}{c}-0.030^{* * *} \\
(0.003)\end{array}$ \\
\hline Squared form & $\begin{array}{c}0.004^{* * *} \\
(0.000)\end{array}$ & $\begin{array}{c}0.006^{* * *} \\
(0.000)\end{array}$ & $\begin{array}{c}0.005^{* * *} \\
(0.000)\end{array}$ & $\begin{array}{c}0.004^{* * *} \\
(0.000)\end{array}$ & $\begin{array}{c}0.004^{* * *} \\
(0.000)\end{array}$ \\
\hline
\end{tabular}




\begin{tabular}{lccccc}
$\begin{array}{l}\text { Average lifetime } \\
\text { earnings of }\end{array}$ & $0.070^{* * *}$ & $0.059^{* * *}$ & $0.092^{* * *}$ & $0.073^{* * *}$ & $0.081^{* * *}$ \\
partner/1000 & & & & & \\
& & & & & \\
\multicolumn{1}{c}{ Squared form } & $-0.011^{* * *}$ & -0.014 & $-0.015^{* * *}$ & $-0.012^{* * *}$ & $-0.014^{* * *}$ \\
& $(0.002)$ & $(0.009)$ & $(0.003)$ & $(0.002)$ & $(0.002)$ \\
Current salary of & $-0.028^{* * *}$ & $-0.045^{* * *}$ & $-0.038^{* * *}$ & $-0.027^{* * *}$ & $-0.030^{* * *}$ \\
partner/1000 & & & & & \\
\multicolumn{1}{c}{ Squared form } & $(0.002)$ & $(0.006)$ & $(0.003)$ & $(0.002)$ & $(0.003)$ \\
& $0.002^{* * *}$ & $0.004^{* * *}$ & $0.003^{* * *}$ & $0.002^{* * *}$ & $0.002^{* * *}$ \\
Observations & $(0.000)$ & $(0.001)$ & $(0.001)$ & $(0.000)$ & $(0.000)$ \\
& 86,666 & 86,666 & 86,666 & 86,666 & 86,666
\end{tabular}

Note: Full sample of person-year observations 2005-2010. Standard errors are in parentheses. For Probit regressions, the table reports marginal effects estimated at the mean. ${ }^{* * *} \mathrm{p}<0.01,{ }^{* *} \mathrm{p}<0.05,{ }^{*} \mathrm{p}<0.1$.

Reaching the statutory eligibility age increases the probability of retirement. This effect hints at the existence of a corner solution for numerous individuals: employment legislation and social protection legislation are such that individuals face hurdles to continuing work after the statutory eligibility age. If individuals did not have to face such hurdles, their choices would likely be smoother with overall later retirement.

Being a woman significantly decreases the probability of retirement. Being older increases the probability to retire but at a progressively decreasing rate. Having a partner has a negative and significant impact on retirement, except in the fixed effect model. Having an active partner decreases the probability of retirement in the fixed effect model. We observe higher instantaneous retirement probabilities in Flanders than in Brussels and Wallonia. Working part-time significantly increases the probability to retire, except in the fixed effect model where the opposite prevails. Both results need not be contradictory. While the part-time variable captures a status in the other models (being a part-time worker), in the fixed effect specification it captures the transition into part-time status 
(shifting into part-time status). Being a white-collar worker and working in the primary sector (as compared to the secondary sector) decreases the retirement probability in every model. Higher average lifetime earnings and higher current salary decrease the probability to retire at an accelerating rate. Finally, the average lifetime earnings of the partner increase the probability to retire at a decelerating rate and the current salary of the partner decreases the probability to retire at an accelerating rate.

In Table 4.3, we report regressions separately for men and women. We only present the results for the probit model in the body of the text for the sake of simplicity and because the probit model presents the best fit in terms of its predictions of retirement rates compared to the observed retirement rates. ${ }^{174} \mathrm{We}$ find a positive and significant effect of ITAX and SSW for both sexes and the effect of ITAX and SSW is slightly stronger for women than for men. For the rest, the results are qualitatively similar to Table 4.2 and we observe stronger effects of age, reaching the statutory eligibility age and the region on the retirement probability of men. Having a partner significantly decreases the retirement probability of men but it significantly increases the retirement probability of women. However, having an active spouse decreases the retirement probability of men but it is not the case for women as it increases their retirement probability. This opposite effect on men and women explains the effect of having an active spouse in table 4.2 that is nonsignificant. The positive effect of having an active spouse for men, and the negative effect for women would indicate that women follow their partner's decision, while men do not follow their partner's. Being a white-collar worker, working part-time and working in the primary sector (as compared to the secondary sector) significantly decreases the retirement probability of men but not of women (part-time work is only significant at the $10 \%$ level for women). Interestingly, the effect of the average lifetime earnings and of the

${ }^{174}$ All results are presented in the appendix. 
current earnings is stronger for women than for men. However, the effect of the average earnings and the current earnings of the partner is stronger for men than for women.

Table 4.3: Retirement using the probit model - by sex

\begin{tabular}{|c|c|c|}
\hline & Male & Female \\
\hline ITAX & $\begin{array}{c}0.061^{* * *} \\
(0.008)\end{array}$ & $\begin{array}{c}0.075^{* * *} \\
(0.008)\end{array}$ \\
\hline SSW/100,000 & $\begin{array}{c}0.158^{* * *} \\
(0.005)\end{array}$ & $\begin{array}{c}0.247^{* * *} \\
(0.008)\end{array}$ \\
\hline Age & $\begin{array}{c}0.610^{* * *} \\
(0.037)\end{array}$ & $\begin{array}{c}0.590^{* * *} \\
(0.049)\end{array}$ \\
\hline Age squared & $\begin{array}{c}-0.005^{* * * *} \\
(0.000)\end{array}$ & $\begin{array}{c}-0.005^{* * *} \\
(0.000)\end{array}$ \\
\hline SEA & $\begin{array}{c}0.470^{* * *} \\
(0.021)\end{array}$ & $\begin{array}{c}0.269^{* * *} \\
(0.026)\end{array}$ \\
\hline Partner & $\begin{array}{c}-0.028^{* * *} \\
(0.005)\end{array}$ & $\begin{array}{c}0.020^{* * *} \\
(0.005)\end{array}$ \\
\hline Active spouse & $\begin{array}{l}0.010^{* *} \\
(0.004)\end{array}$ & $\begin{array}{c}-0.022^{* * *} \\
(0.005)\end{array}$ \\
\hline Brussels & $\begin{array}{c}-0.028^{* * *} \\
(0.005)\end{array}$ & $\begin{array}{l}-0.011^{*} \\
(0.006)\end{array}$ \\
\hline Wallonia & $\begin{array}{c}-0.012^{* * *} \\
(0.003)\end{array}$ & $\begin{array}{l}-0.010^{* *} \\
(0.004)\end{array}$ \\
\hline Part-time & $\begin{array}{c}0.028^{* * *} \\
(0.004)\end{array}$ & $\begin{array}{l}0.008^{*} \\
(0.004)\end{array}$ \\
\hline White collar & $\begin{array}{c}-0.051^{* * *} \\
(0.004)\end{array}$ & $\begin{array}{l}-0.007 \\
(0.005)\end{array}$ \\
\hline Primary sector & $\begin{array}{c}-0.043^{* * *} \\
(0.013)\end{array}$ & $\begin{array}{c}0.010 \\
(0.038)\end{array}$ \\
\hline Tertiary sector & $\begin{array}{c}0.008^{* * *} \\
(0.003)\end{array}$ & $\begin{array}{c}-0.011^{* * *} \\
(0.004)\end{array}$ \\
\hline $\begin{array}{l}\text { Average salary } \\
\text { of the reference } \\
\text { person/1000 }\end{array}$ & $-0.201^{* * *}$ & $-0.309^{* * *}$ \\
\hline Squared form & $\begin{array}{c}(0.012) \\
0.019^{* * *} \\
(0.002)\end{array}$ & $\begin{array}{c}(0.021) \\
0.020^{* * * *} \\
(0.005)\end{array}$ \\
\hline
\end{tabular}




\begin{tabular}{|c|c|c|}
\hline $\begin{array}{l}\text { Current salary of } \\
\text { the reference } \\
\text { person/1000 }\end{array}$ & $-0.021^{* * *}$ & $-0.055^{* * *}$ \\
\hline Squared form & $\begin{array}{c}(0.004) \\
0.003^{* * * *} \\
(0.000)\end{array}$ & $\begin{array}{c}(0.006) \\
0.011^{* * *} \\
(0.001)\end{array}$ \\
\hline $\begin{array}{l}\text { Average salary } \\
\text { of the } \\
\text { partner/1000 }\end{array}$ & $0.090^{* * *}$ & $0.028^{* * *}$ \\
\hline Squared form & $\begin{array}{c}(0.010) \\
-0.014^{* *} \\
(0.006)\end{array}$ & $\begin{array}{l}(0.007) \\
-0.003 \\
(0.003)\end{array}$ \\
\hline $\begin{array}{l}\text { Current salary of } \\
\text { the partner/1000 }\end{array}$ & $\begin{array}{c}-0.037^{* * *} \\
(0.005)\end{array}$ & $\begin{array}{r}-0.011^{* * *} \\
(0.003)\end{array}$ \\
\hline Squared form & $\begin{array}{c}0.002 \\
(0.002)\end{array}$ & $\begin{array}{l}0.001^{* *} \\
(0.000)\end{array}$ \\
\hline Observations & 56,377 & 30,289 \\
\hline
\end{tabular}

Note: Full sample of person-year observations 20052010. Standard errors are in parentheses. The table reports marginal effects estimated at the mean. ${ }^{* * *} \mathrm{p}<0.01,{ }^{* *} \mathrm{p}<0.05,{ }^{*} \mathrm{p}<0.1$.

\subsection{SIMULATIONS}

In order to illustrate the effect of incentive measures on the probability to exit the labor market, we present the results of counterfactual simulations. In this exercise, we rely on the regression results of Table 4.2 using the probit model. Figure 4.11 plots separately the actual exit rates and the predictions by the model - by sex and age. We find an increasing probability of retirement over age, that spikes at the statutory eligibility age of the oldage pension system. However, our predictions do not fully capture the spikes that appear at the early eligibility age of the old-age pension (60). 
Figure 4.11: Retirement rate for men and women- actual and predicted baseline
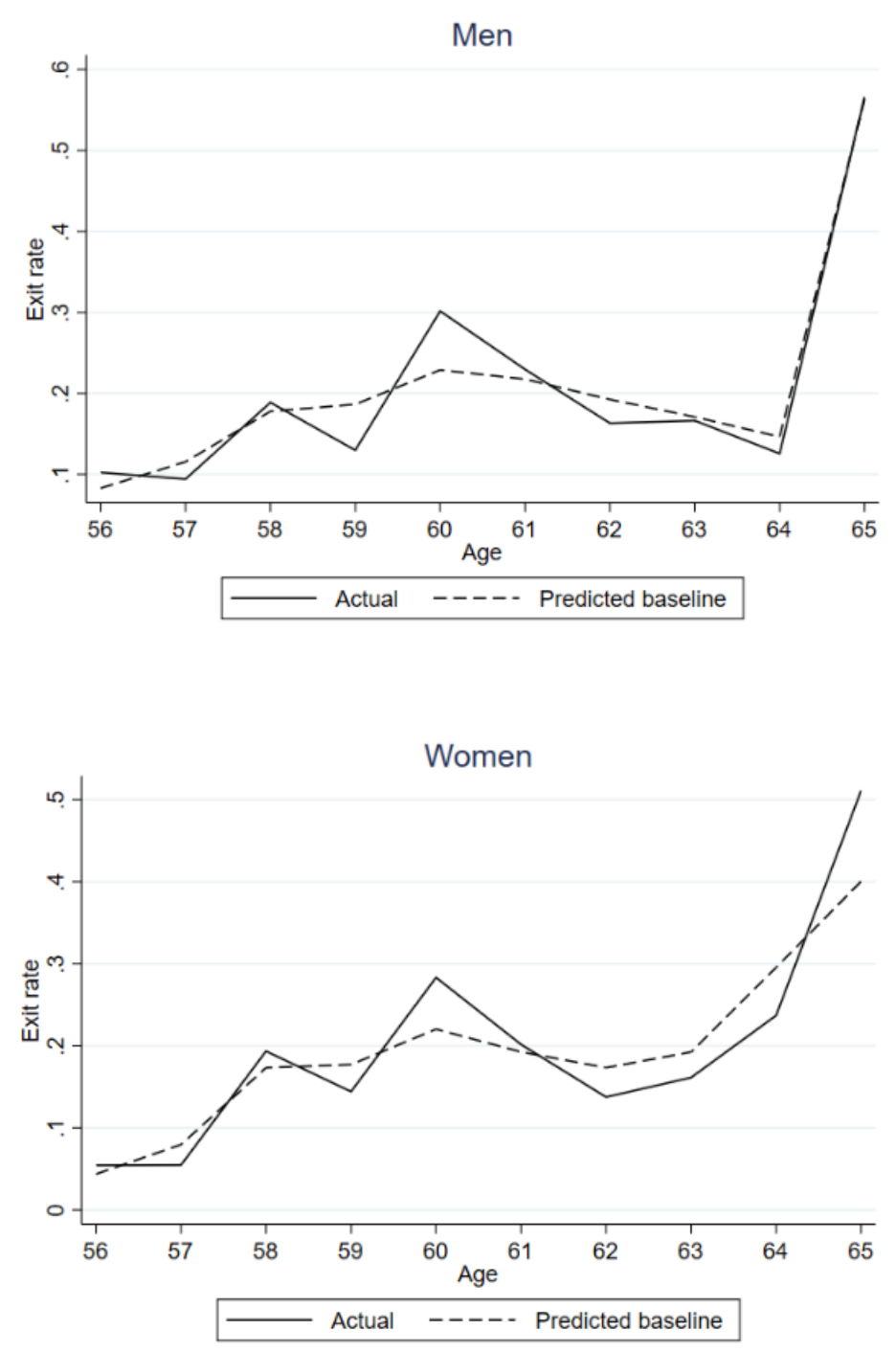

Author's calculations based on CBSS data.

In a second step, we use these predictions as a baseline for our counterfactual simulations. In this simulation, we predict the value of the incentive measures that would have prevailed if the social security schemes had remained unreformed and in their 2004 format. The reforms include the delaying of the statutory eligibility age old-age pension for women in 2006 and 2009, the one-year increase in the career conditions for the early eligibility age old-age pension in 2005 and the introduction of the pension bonus in 2007 . 
All other covariates are kept at their actual value. The aim of such a counterfactual simulation is to eradicate all changes due to policy reforms and to examine what would have been the exit rate of older Belgian workers without the reforms that happened between 2005 and 2010.

Figure 4.12 presents the average probability of retirement for the total population aged 56-65 for years 2005 to 2010 . We observe that the counterfactual is higher than the baseline for every year of observation, which means that the reforms implemented during these years have been efficient in decreasing opportunity to exit the labor market. However, these averages can hide different situations with respect to age. In the following figures we show the evolution of the exit rate by sex and year for certain key eligibility ages.

Figures 4.13 shows simulated counterfactual exit rates for men aged 60 and 65 . We see that for men aged 60, the counterfactual is higher than the baseline for every year of observation, indicating that the reform that increased the career conditions for the early eligibility of the old-age pension has fulfilled its role of closing the early exit through the old-age pension. ${ }^{175}$ Nevertheless, we observe an increase in the retirement rate of the baseline scenario starting in 2008, while the counterfactual simulation displays a decreasing retirement rate. This is due to the introduction of the pension bonus with an effective date in 2008, granted for continued work after age 62 or 44 years of career. The bonus directly increases the SSW with its strongly positive sign, leading to higher counterfactual exit rates through an income effect. The dynamic incentive effect through a reduced ITAX that would lead to lower retirement rates remains moderate as we find that the SSW has a stronger impact on the retirement probability than the ITAX does (see table 4.3). For men at retirement age 65, while the counterfactual is higher during the first

\footnotetext{
175 In 2010, the average years of career per worker aged 60 decreases in our sample compared to previous years, leading to a decrease in the simulated ITAX and thus a decrease in the predicted retirement rate of the counterfactual simulation.
} 
years of simulation, the baseline dominates in all later years due to the introduction of the pension bonus. This result contradicts the simulation of incentive measures in chapter three, in which the pension bonus was expected to create an incentive to stay on the labor market through a reduced ITAX effect.

Figure 4.12: Counterfactual simulation - total population aged 56-65
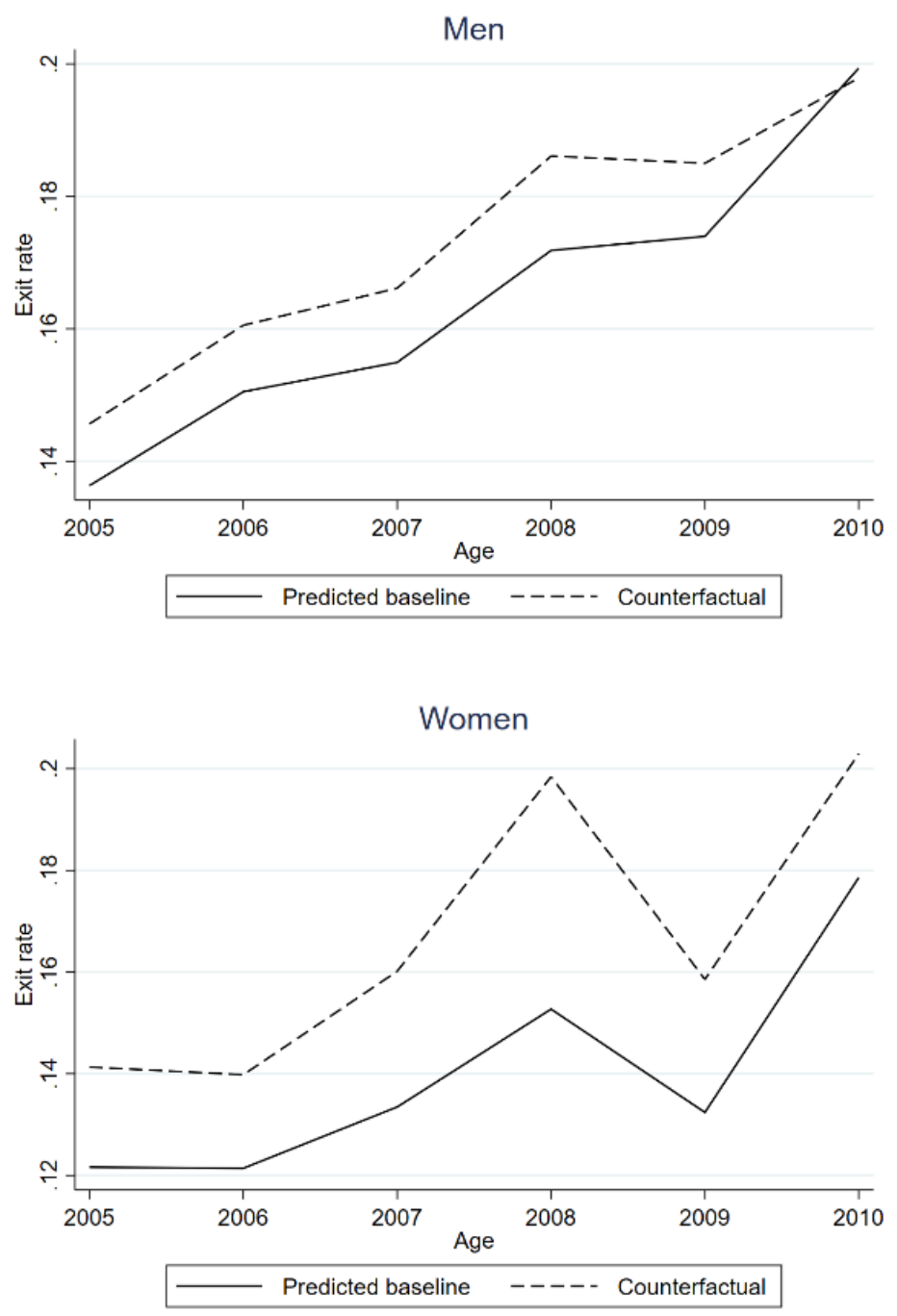

Author's calculations based on CBSS data. 
Figure 4.13: Counterfactual simulation- Men aged 60 and 65 - by year
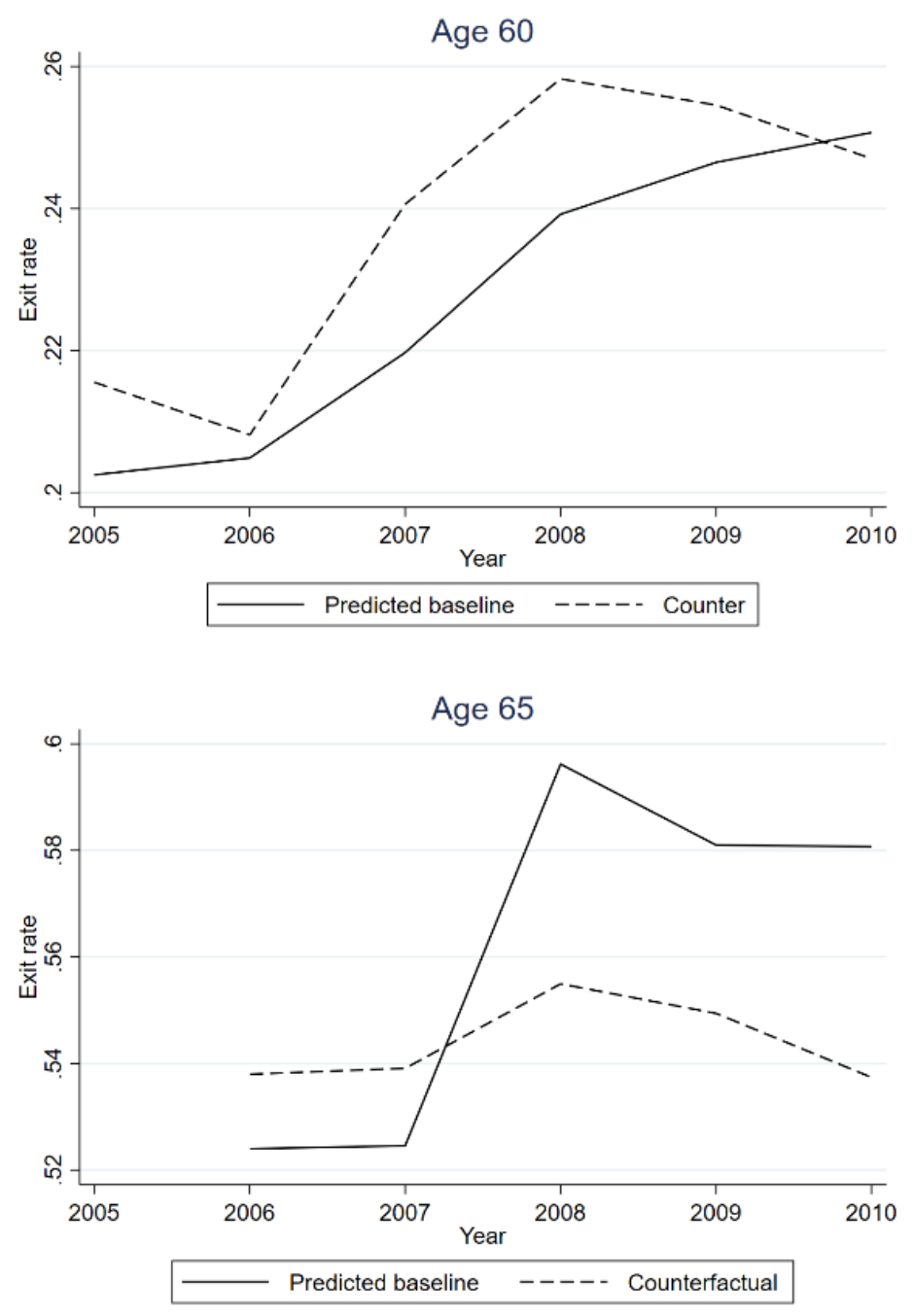

Note: The baseline is obtained from the regressions from Table 2 using the ITAX specification and the probit model. Due to the structure of the dataset, no one is aged 65 in 2005. Author's calculations based on CBSS data.

Figure 4.14 presents the simulations of the baseline and the counterfactual scenarios for women at ages $60,63,64$ and 65 where we vary the ITAX and the SSW measures and distinguish the effect of the statutory eligibility age changes. Ages 63 to 65 correspond to the statutory eligibility age for the successive cohorts under analysis. Looking at the simulation for women aged 60 , we observe similar results as for men. The counterfactual simulation shows that the retirement rates would have been higher without the reform that increased the career conditions for the early eligibility for the old-age pension system. 
Here however, the effect of the pension bonus is not as strong as it was for men because women have shorter careers on average and were thus less likely to satisfy the eligibility conditions for the pension bonus at age 60 . At the age of 65 , as for the men, we observe the impact of the pension bonus as a result of the increase of SSW.

At ages 63 and 64, in addition to the ITAX and the SSW variable the statutory eligibility variable matters in the counterfactual simulation. When taking statutory eligibility age explicitly into the analysis, we document the key role of eligibility variables. There are important decreases in the retirement probability of women of the baseline simulation as compared to the counterfactual including the statutory eligibility age dummy at the age of 63 in 2006 and 64 in 2009. These simulations indicate that the 1997 reform that equalized the statutory eligibility age of men and women fulfilled its role of limiting the access of women to the old-age pension system before the statutory eligibility age, mostly through a gate-keeping logic rather than through financial incentives themselves in line with Jousten and Tarantchenko (2014). 
Figure 4.14: Counterfactual simulation without SEA change- Women - by year
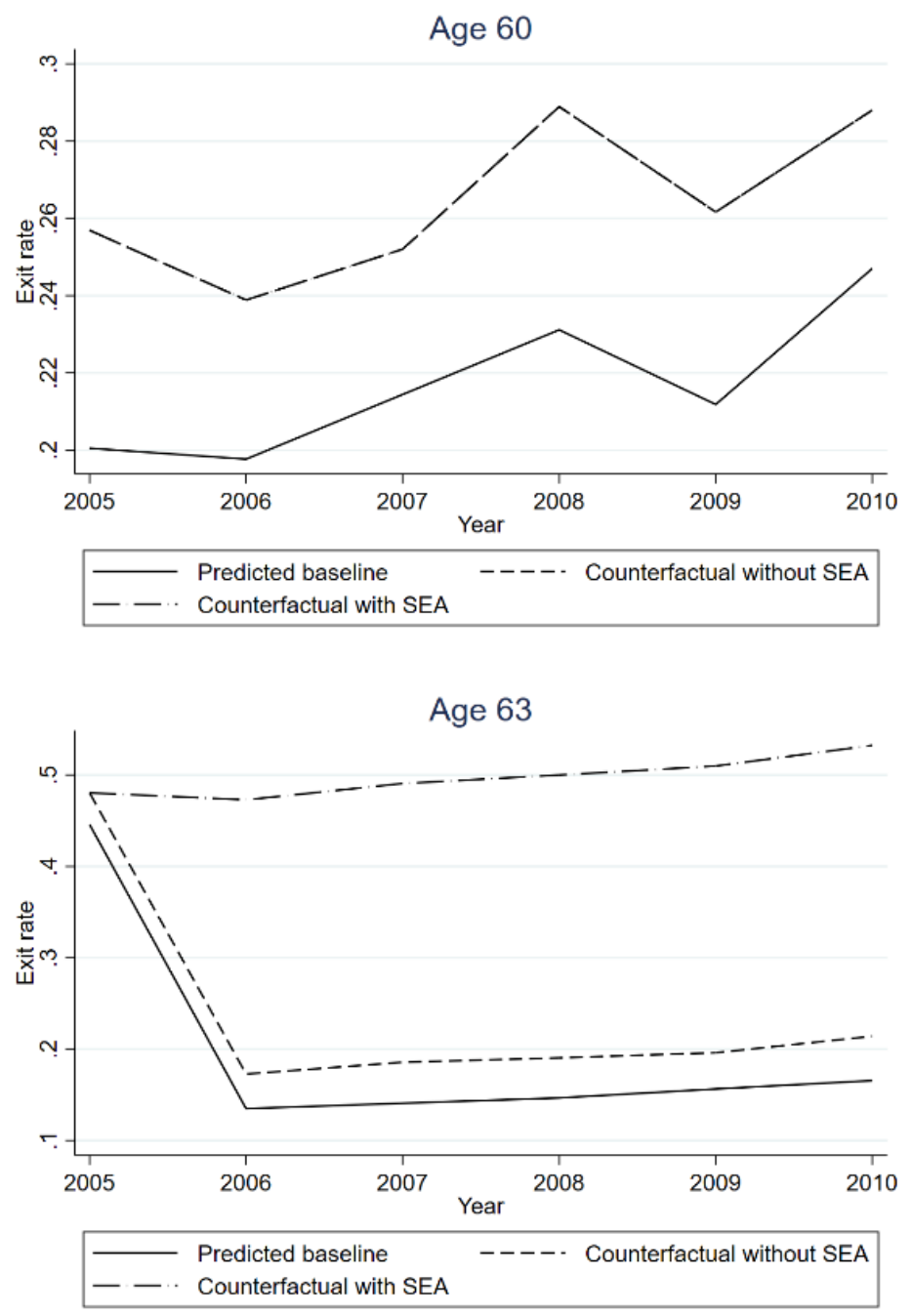

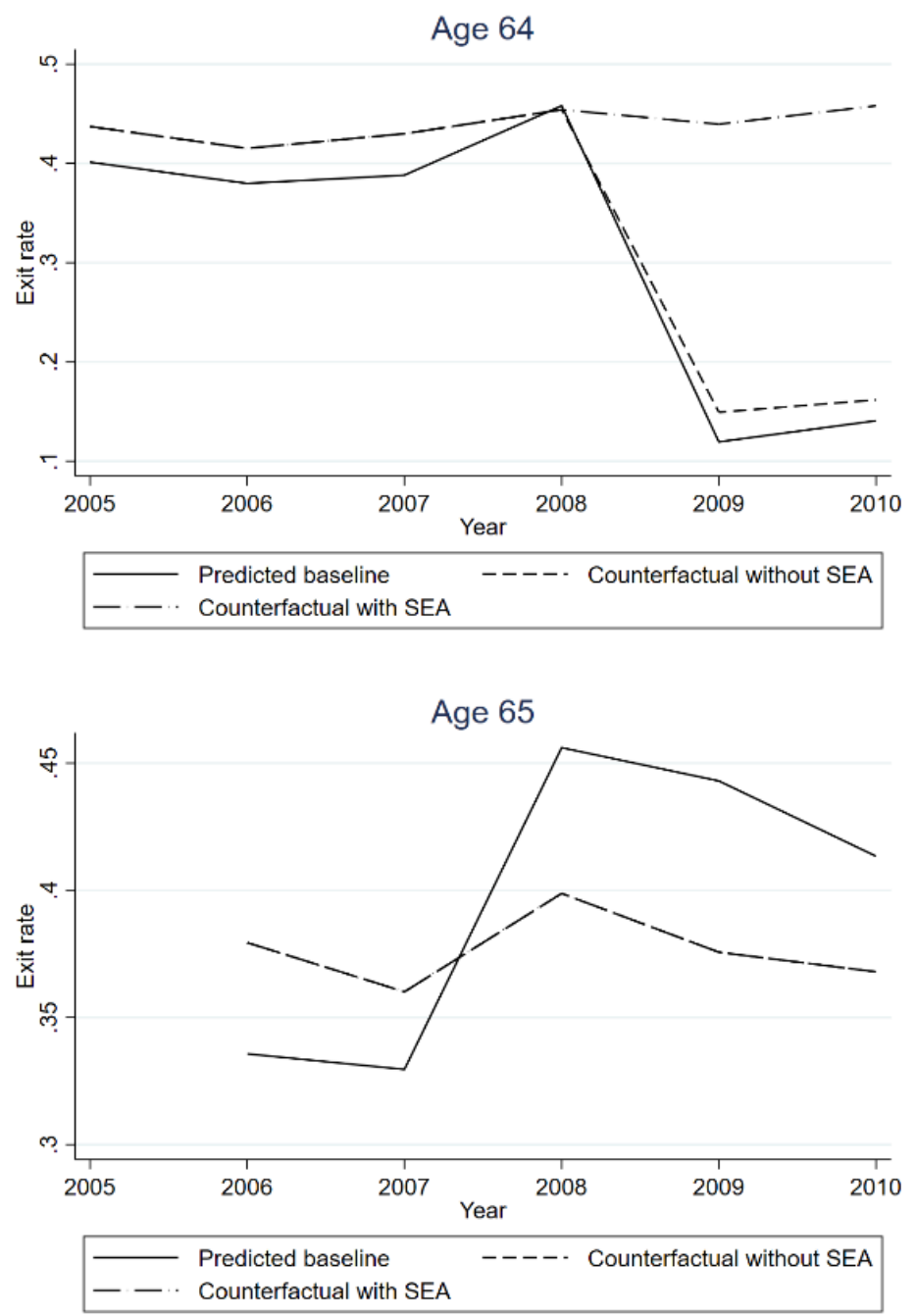

Note: The baseline is obtained from the regressions from Table 2 using the ITAX specification and the probit model. Coincidentally, no sample-member is aged 65 in 2005.

Author's calculations based on CBSS data.

Finally, in Figure 4.15, we present the employment survival curves according to the predicted probability of exit and our counterfactual simulation. These curves show the proportion of workers who remain in employment between ages 56 and 65 if we start with a fully employed population at age 56. We compare the situations in 2005 and 2010 for which we have data with the counterfactual of 2010 in which no reforms happened between 2005 and 2010. We observe that the reforms have been effective in keeping 
workers in employment since both the 2005 and the counterfactual display lower cumulative probability to be active.

Figure 4.15: Employment survival curve

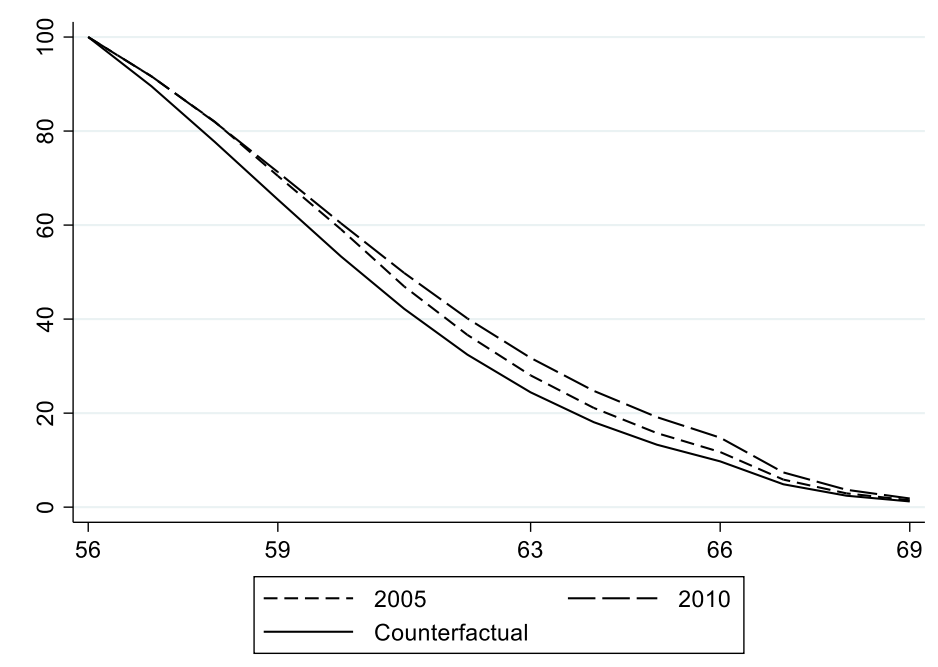

Author's calculations based on CBSS data.

\subsection{CONCLUSION}

In this chapter, we explore the link between social security incentives and labor supply using data from an administrative panel data set. Following a set of Belgian workers from the private sector for at most ten years, we calculate how the various reforms implemented during the period have affected the financial incentives to retire from the labor market.

We consider both the wealth effect as captured by the Social Security Wealth and the dynamic incentive effect when changing retirement behavior at the margin (social security accrual and implicit tax on continued activity). Given the Belgian landscape that offers older workers several possibilities of exiting, we aggregate the social security scheme specific measures into one single incentive indicator. 
Our general regression analysis shows that the effect of SSW and ITAX are positive, for example more generous measures induce higher retirement rates. When we consider men and women separately, we find a stronger effect of the dynamic incentive (ITAX) and SSW for women.

Based on these results we perform a series of simulations in which we neutralize the various reforms that took place since the beginning of our period of observation. While the overarching guiding principle behind these reforms was to increase older workers' labor force participation, they were rather different in their effects on incentive variables: the tightening of eligibility conditions unambiguously led to lower labor market retirement rates, the introduction of the pension bonus for continued work led to a positive wealth effect - hence increasing retirement hazards. Our results are ambiguous, with strongly incentivizing effects at lower ages and more mixed results at higher ages particularly for men. From a policy perspective, the abolition of the pension bonus in 2015 can be considered as a consistent measure if the primary aim of successive governments was the increase of the effective retirement age. Also, closing early entitlement routes remains a powerful policy tool - as illustrated by the lower retirement hazards in the baseline versus the counterfactual situation. 


\section{REFERENCES}

Dellis, A., R. Desmet, A. Jousten, and S. Perelman (2004). Micro- Modeling of Retirement in Belgium. In Social Security Programs and Retirement around the World: MicroEstimation, edited by J. Gruber and D. Wise, 41-98. Chicago: University of Chicago Press.

Eurostat, (2019). European Labor Force Survey, European Union, Luxembourg.

Fraikin, A.L., Jousten, A., and Lefèbvre, M. (2020). Social Security Incentives for Retirement in Belgium: An analysis of four decades of change. In Börsch-Supan, A. and Coile, C., Social security around the world. Chicago: University of Chicago Press and NBER.

Jousten A. and M. Lefebvre (2013). Retirement Incentives in Belgium: Estimations and Simulations Using SHARE Data, De Economist, vol. 161(3), pages 253-276.

Jousten A. and M. Lefebvre (2019). Spousal and survivor benefits in option value models of retirement: an application to Belgium, Journal of Pension Economics and Finance, vol. 18(1), pages 66-87.

Jousten, A. and M. Lefebvre (2016). Older men's labor force participation in Belgium, eWorking Paper University of Liège, forthcoming in D. Wise, Social Security and Retirement around the World: men's labor force participation, University of Chicago Press and NBER.

Jousten, A., Perelman, S., Sigismondi, F., and Tarantchenko, E. (2012). Accrued Pension Rights in Belgium: Micro-Simulation of Reforms. International Journal of Microsimulation, 5(2), 22-39. 
Jousten, A. and Tarantchenko, E. (2014). New evidence on the social security incentives as drivers of retirement behavior. SSRN Electronic Journal. 10.2139/ssrn.2429287.

Pestieau P. and J.P Stijns (1999).Social Security and Retirement in Belgium, in Social Security and Retirement around the World, edited by J. Gruber and D. Wise, National Bureau of Economic Research.

Seibold, A. (2017) Reference Dependence in Retirement Behavior: Evidence from German Pension Discontinuities. mimeo, London School of Economics. 


\section{APPENDIX}

4.A.1. AGGREGATION WEIGHTS BY EXIT PATHWAYS FOR MEN (AVERAGED OVER YEARS)

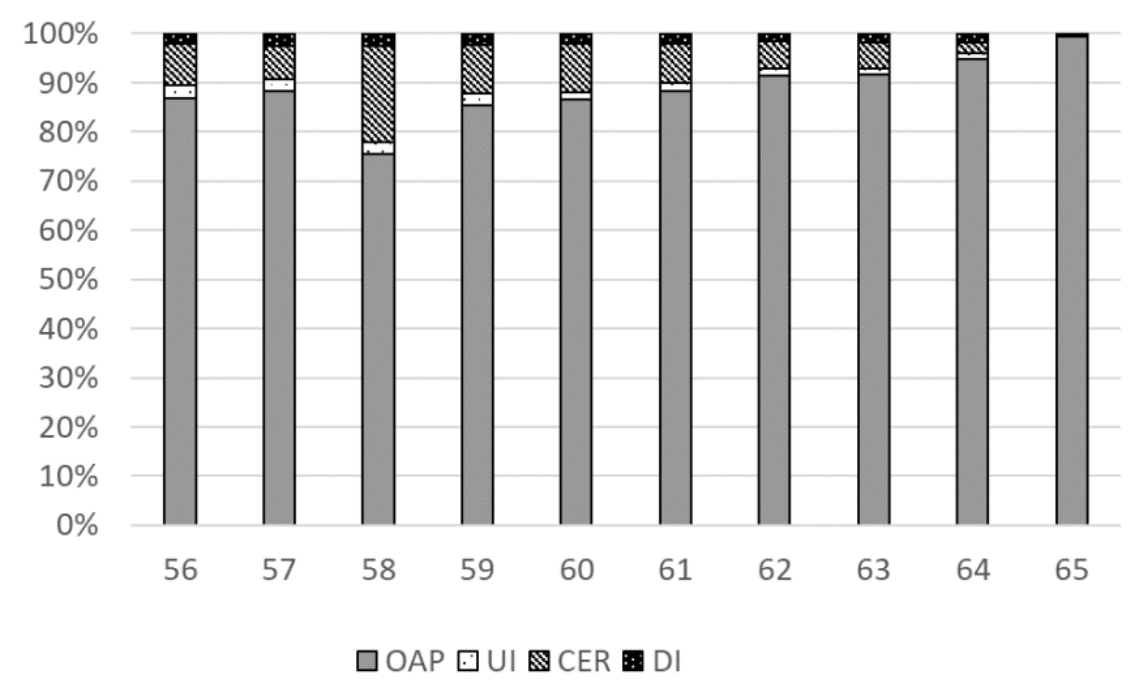

4.A.2. AGGREGATION WEIGHTS BY EXIT PATHWAYS FOR WOMEN (AVERAGED OVER YEARS)

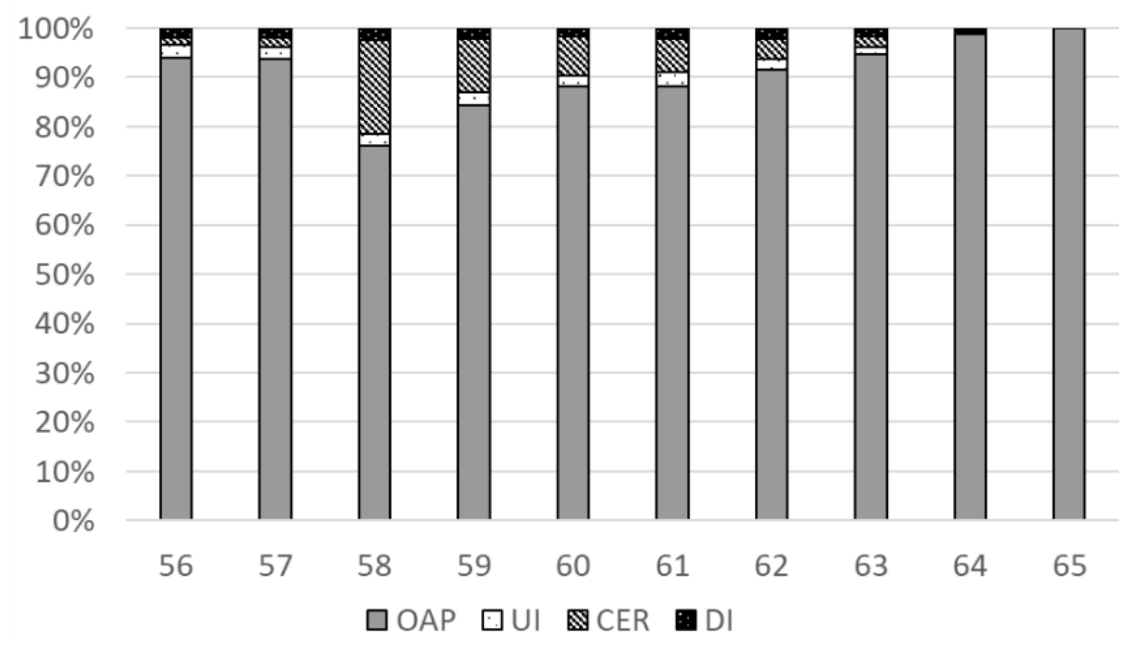


4.A.3. REGRESSION RESULTS - TOTAL SAMPLE WITH ACCRUAL

\begin{tabular}{|c|c|c|c|c|c|}
\hline & LPM & Fixed effect & $\begin{array}{c}\text { Random } \\
\text { effect }\end{array}$ & Probit & $\begin{array}{l}\text { Probit } \\
\text { random } \\
\text { effects }\end{array}$ \\
\hline \multirow{2}{*}{$\begin{array}{l}\text { Accrual/ } \\
100,000\end{array}$} & $-0.191^{* * *}$ & $-0.290^{* * *}$ & $-0.135^{* * *}$ & $-0.283^{* * *}$ & $-0.293^{* * *}$ \\
\hline & $(0.017)$ & $(0.020)$ & $(0.017)$ & $(0.022)$ & $(0.023)$ \\
\hline SSW/100,000 & $\begin{array}{c}0.161^{* * *} \\
(0.004)\end{array}$ & $\begin{array}{c}-0.155^{* * *} \\
(0.015)\end{array}$ & $\begin{array}{c}0.207^{* * *} \\
(0.006)\end{array}$ & $\begin{array}{c}0.168^{* * *} \\
(0.004)\end{array}$ & $\begin{array}{c}0.184^{* * *} \\
(0.002)\end{array}$ \\
\hline Female & $\begin{array}{c}-0.059^{* * *} \\
(0.003)\end{array}$ & & $\begin{array}{c}-0.079^{* * *} \\
(0.005)\end{array}$ & $\begin{array}{c}-0.057^{* * *} \\
(0.003)\end{array}$ & $\begin{array}{c}-0.065^{* * *} \\
(0.004)\end{array}$ \\
\hline Age & $\begin{array}{c}0.588^{* * *} \\
(0.029)\end{array}$ & $\begin{array}{c}0.482^{* * *} \\
(0.037)\end{array}$ & $\begin{array}{c}0.428^{* * * *} \\
(0.029)\end{array}$ & $\begin{array}{c}0.622^{* * *} \\
(0.029)\end{array}$ & $\begin{array}{c}0.642^{* * *} \\
(0.030)\end{array}$ \\
\hline Age squared & $\begin{array}{c}-0.005^{* * *} \\
(0.000)\end{array}$ & $\begin{array}{c}-0.004^{* * *} \\
(0.000)\end{array}$ & $\begin{array}{c}-0.003^{* * *} \\
(0.000)\end{array}$ & $\begin{array}{c}-0.005^{* * *} \\
(0.000)\end{array}$ & $\begin{array}{c}-0.005^{* * *} \\
(0.000)\end{array}$ \\
\hline SEA & $\begin{array}{c}0.362^{* * *} \\
(0.010)\end{array}$ & $\begin{array}{c}0.422^{* * *} \\
(0.011)\end{array}$ & $\begin{array}{c}0.347^{* * * *} \\
(0.010)\end{array}$ & $\begin{array}{c}0.386^{* * *} \\
(0.016)\end{array}$ & $\begin{array}{c}0.251^{* * *} \\
(0.009)\end{array}$ \\
\hline Partner & $\begin{array}{c}-0.012^{* * *} \\
(0.003)\end{array}$ & $\begin{array}{c}0.000 \\
(0.015)\end{array}$ & $\begin{array}{c}-0.016^{* * *} \\
(0.005)\end{array}$ & $\begin{array}{c}-0.018^{* * *} \\
(0.003)\end{array}$ & $\begin{array}{c}-0.019^{* * *} \\
(0.004)\end{array}$ \\
\hline Active partner & $\begin{array}{c}0.000 \\
(0.003)\end{array}$ & $\begin{array}{c}-0.017^{* * *} \\
(0.005)\end{array}$ & $\begin{array}{c}0.005 \\
(0.004)\end{array}$ & $\begin{array}{c}0.004 \\
(0.003)\end{array}$ & $\begin{array}{l}0.005^{*} \\
(0.003)\end{array}$ \\
\hline Brussels & $\begin{array}{c}-0.021^{* * *} \\
(0.004)\end{array}$ & & $\begin{array}{c}-0.036^{* * *} \\
(0.007)\end{array}$ & $\begin{array}{c}-0.020^{* * *} \\
(0.004)\end{array}$ & $\begin{array}{c}-0.023^{* * *} \\
(0.005)\end{array}$ \\
\hline Wallonia & $\begin{array}{c}-0.012^{* * *} \\
(0.003)\end{array}$ & & $\begin{array}{c}-0.017^{* * *} \\
(0.004)\end{array}$ & $\begin{array}{c}-0.011^{* * *} \\
(0.003)\end{array}$ & $\begin{array}{c}-0.012^{* * *} \\
(0.003)\end{array}$ \\
\hline Part-time & $\begin{array}{c}0.021^{* * *} \\
(0.003)\end{array}$ & $\begin{array}{c}-0.064^{* * *} \\
(0.006)\end{array}$ & $\begin{array}{c}0.004 \\
(0.004)\end{array}$ & $\begin{array}{c}0.021^{* * *} \\
(0.003)\end{array}$ & $\begin{array}{c}0.021^{* * * *} \\
(0.003)\end{array}$ \\
\hline White collar & $\begin{array}{c}-0.033^{* * *} \\
(0.003)\end{array}$ & $\begin{array}{c}-0.081^{* * *} \\
(0.024)\end{array}$ & $\begin{array}{c}-0.049^{* * *} \\
(0.005)\end{array}$ & $\begin{array}{c}-0.032^{* * *} \\
(0.003)\end{array}$ & $\begin{array}{c}-0.035^{* * *} \\
(0.003)\end{array}$ \\
\hline Primary sector & $\begin{array}{c}-0.037^{* * *} \\
(0.014)\end{array}$ & & $\begin{array}{c}-0.056^{* *} \\
(0.023)\end{array}$ & $\begin{array}{c}-0.035^{* * *} \\
(0.011)\end{array}$ & $\begin{array}{c}-0.043^{* * *} \\
(0.015)\end{array}$ \\
\hline Tertiary sector & $\begin{array}{c}0.004 \\
(0.002)\end{array}$ & & $\begin{array}{c}0.020^{* * * *} \\
(0.004)\end{array}$ & $\begin{array}{c}0.002 \\
(0.002)\end{array}$ & $\begin{array}{c}0.003 \\
(0.003)\end{array}$ \\
\hline \multirow[t]{2}{*}{$\begin{array}{l}\text { Average salary } \\
\text { of the reference } \\
\text { person/1000 }\end{array}$} & $-0.183^{* * *}$ & $-2.170^{* * *}$ & $-0.254^{* * *}$ & $-0.198^{* * *}$ & $-0.221^{* * *}$ \\
\hline & $(0.010)$ & $(0.145)$ & $(0.014)$ & $(0.010)$ & $(0.012)$ \\
\hline Squared form & $0.017^{* * *}$ & $0.293^{* * *}$ & $0.028^{* * *}$ & $0.017^{* * *}$ & $0.019^{* * *}$ \\
\hline
\end{tabular}




\begin{tabular}{|c|c|c|c|c|c|}
\hline & $(0.002)$ & $(0.017)$ & $(0.003)$ & $(0.002)$ & $(0.002)$ \\
\hline \multirow{2}{*}{$\begin{array}{l}\text { Current salary } \\
\text { of the reference } \\
\text { person/1000 }\end{array}$} & $-0.031^{* * *}$ & $-0.089^{* * *}$ & $-0.053^{* * *}$ & $-0.026^{* * *}$ & $-0.028^{* * *}$ \\
\hline & $(0.003)$ & $(0.006)$ & $(0.004)$ & $(0.003)$ & $(0.003)$ \\
\hline \multirow[t]{2}{*}{ Squared form } & $0.004^{* * *}$ & $0.006^{* * *}$ & $0.005^{* * *}$ & $0.003^{* * *}$ & $0.004^{* * *}$ \\
\hline & $(0.000)$ & $(0.000)$ & $(0.000)$ & $(0.000)$ & $(0.000)$ \\
\hline \multirow{2}{*}{$\begin{array}{l}\text { Average salary } \\
\text { of the } \\
\text { partner/1000 }\end{array}$} & $0.067^{* * *}$ & $0.060^{* * * *}$ & $0.089^{* * *}$ & $0.069^{* * *}$ & $0.075^{* * *}$ \\
\hline & $(0.006)$ & $(0.022)$ & $(0.008)$ & $(0.005)$ & $(0.006)$ \\
\hline \multirow[t]{2}{*}{ Squared form } & $-0.010^{* * *}$ & -0.014 & $-0.014^{* * *}$ & $-0.011^{* * *}$ & $-0.012^{* * *}$ \\
\hline & $(0.002)$ & $(0.009)$ & $(0.003)$ & $(0.002)$ & $(0.002)$ \\
\hline \multirow{2}{*}{$\begin{array}{l}\text { Current salary } \\
\text { of the } \\
\text { partner/1000 }\end{array}$} & $-0.027^{* * *}$ & $-0.045^{* * *}$ & $-0.038^{* * *}$ & $-0.026^{* * *}$ & $-0.103^{* * *}$ \\
\hline & $(0.002)$ & $(0.006)$ & $(0.003)$ & $(0.002)$ & $(0.003)$ \\
\hline \multirow[t]{2}{*}{ Squared form } & $0.002^{* * *}$ & $0.004^{* * *}$ & $0.003^{* * *}$ & $0.002^{* * *}$ & $0.002^{* * *}$ \\
\hline & $(0.000)$ & $(0.001)$ & $(0.001)$ & $(0.000)$ & $(0.000)$ \\
\hline Observations & 86,666 & 86,666 & 86,666 & 86,666 & 86,666 \\
\hline
\end{tabular}

Note: Full sample of person-year observations 2005-2010. Standard errors are in parentheses. For Probit regressions, the table reports marginal effects estimated at the mean. ${ }^{* *} \mathrm{p}<0.01,{ }^{* *} \mathrm{p}<0.05,{ }^{*} \mathrm{p}<0.1$. 


\section{A.4 REGRESSION RESULTS - MEN WITH ITAX}

\begin{tabular}{|c|c|c|c|c|c|}
\hline & LPM & Fixed effect & $\begin{array}{c}\text { Random } \\
\text { effect }\end{array}$ & Probit & $\begin{array}{l}\text { Probit } \\
\text { random } \\
\text { effects }\end{array}$ \\
\hline Accrual/ 100,000 & $\begin{array}{c}0.075^{* * *} \\
(0.007)\end{array}$ & $\begin{array}{c}0.146^{* * *} \\
(0.008)\end{array}$ & $\begin{array}{c}0.081^{* * *} \\
(0.007)\end{array}$ & $\begin{array}{c}0.061^{* * *} \\
(0.008)\end{array}$ & $\begin{array}{c}0.067^{* * *} \\
(0.008)\end{array}$ \\
\hline SSW/100,000 & $\begin{array}{c}0.143^{* * *} \\
(0.005)\end{array}$ & $\begin{array}{c}-0.226^{* * *} \\
(0.017)\end{array}$ & $\begin{array}{c}0.179^{* * *} \\
(0.007)\end{array}$ & $\begin{array}{l}0.158^{* * *} \\
(0.005)\end{array}$ & $\begin{array}{c}0.182^{* * *} \\
(0.007)\end{array}$ \\
\hline Age & $\begin{array}{c}0.578^{* * *} \\
(0.036)\end{array}$ & $\begin{array}{c}0.643^{* * *} \\
(0.045)\end{array}$ & $\begin{array}{c}0.471^{* * *} \\
(0.036)\end{array}$ & $\begin{array}{c}0.610^{* * *} \\
(0.037)\end{array}$ & $\begin{array}{c}0.654^{* * * *} \\
(0.039)\end{array}$ \\
\hline Age squared & $\begin{array}{c}-0.005^{* * *} \\
(0.000)\end{array}$ & $\begin{array}{c}-0.005^{* * *} \\
(0.000)\end{array}$ & $\begin{array}{c}-0.004^{* * *} \\
(0.000)\end{array}$ & $\begin{array}{c}-0.005^{* * *} \\
(0.000)\end{array}$ & $\begin{array}{c}-0.005^{* * *} \\
(0.000)\end{array}$ \\
\hline SEA & $\begin{array}{c}0.437^{* * *} \\
(0.014)\end{array}$ & $\begin{array}{c}0.441^{* * *} \\
(0.013)\end{array}$ & $\begin{array}{c}0.413^{* * *} \\
(0.013)\end{array}$ & $\begin{array}{c}0.470^{* * *} \\
(0.021)\end{array}$ & $\begin{array}{c}0.311^{* * *} \\
(0.013)\end{array}$ \\
\hline Partner & $\begin{array}{c}-0.020^{* * *} \\
(0.004)\end{array}$ & $\begin{array}{l}-0.002 \\
(0.016)\end{array}$ & $\begin{array}{c}-0.025^{* * *} \\
(0.006)\end{array}$ & $\begin{array}{c}-0.028^{* * *} \\
(0.005)\end{array}$ & $\begin{array}{c}-0.032^{* * *} \\
(0.005)\end{array}$ \\
\hline Active partner & $\begin{array}{c}0.006 \\
(0.004)\end{array}$ & $\begin{array}{c}-0.020^{* * *} \\
(0.007)\end{array}$ & $\begin{array}{l}0.011^{* *} \\
(0.005)\end{array}$ & $\begin{array}{l}0.010^{* *} \\
(0.004)\end{array}$ & $\begin{array}{c}0.013^{* * *} \\
(0.004)\end{array}$ \\
\hline Brussels & $\begin{array}{c}-0.028^{* * *} \\
(0.006)\end{array}$ & & $\begin{array}{c}-0.049^{* * * *} \\
(0.009)\end{array}$ & $\begin{array}{c}-0.028^{* * *} \\
(0.005)\end{array}$ & $\begin{array}{c}-0.035^{* * *} \\
(0.007)\end{array}$ \\
\hline Wallonia & $\begin{array}{c}-0.013^{* * *} \\
(0.003)\end{array}$ & & $\begin{array}{c}-0.019^{* * * *} \\
(0.005)\end{array}$ & $\begin{array}{c}-0.012^{* * *} \\
(0.003)\end{array}$ & $\begin{array}{c}-0.013^{* * *} \\
(0.004)\end{array}$ \\
\hline Part-time & $\begin{array}{c}0.027^{* * *} \\
(0.004)\end{array}$ & $\begin{array}{c}-0.075^{* * *} \\
(0.008)\end{array}$ & $\begin{array}{c}0.007 \\
(0.005)\end{array}$ & $\begin{array}{c}0.028^{* * *} \\
(0.004)\end{array}$ & $\begin{array}{c}0.027^{* * *} \\
(0.004)\end{array}$ \\
\hline White collar & $\begin{array}{c}-0.051^{* * *} \\
(0.004)\end{array}$ & $\begin{array}{c}-0.099^{* * *} \\
(0.031)\end{array}$ & $\begin{array}{c}-0.077^{* * *} \\
(0.006)\end{array}$ & $\begin{array}{c}-0.051^{* * *} \\
(0.004)\end{array}$ & $\begin{array}{c}-0.058^{* * *} \\
(0.004)\end{array}$ \\
\hline Primary sector & $\begin{array}{c}-0.044^{* * *} \\
(0.016)\end{array}$ & & $\begin{array}{c}-0.067^{* * *} \\
(0.025)\end{array}$ & $\begin{array}{c}-0.043^{* * *} \\
(0.013)\end{array}$ & $\begin{array}{c}-0.055^{* * *} \\
(0.018)\end{array}$ \\
\hline Tertiary sector & $\begin{array}{c}0.009^{* * *} \\
(0.003)\end{array}$ & & $\begin{array}{c}0.031^{* * *} \\
(0.005)\end{array}$ & $\begin{array}{c}0.008^{* * *} \\
(0.003)\end{array}$ & $\begin{array}{c}0.011^{* * *} \\
(0.003)\end{array}$ \\
\hline $\begin{array}{l}\text { Average salary } \\
\text { of the reference } \\
\text { person/1000 }\end{array}$ & $-0.176^{* * *}$ & $-1.091^{* * *}$ & $-0.231^{* * *}$ & $-0.201^{* * *}$ & $-0.235^{* * *}$ \\
\hline Squared form & $\begin{array}{c}(0.012) \\
0.017^{* * *} \\
(0.002)\end{array}$ & $\begin{array}{c}(0.192) \\
0.175^{* * *} \\
(0.021)\end{array}$ & $\begin{array}{c}(0.017) \\
0.027^{* * *} \\
(0.003)\end{array}$ & $\begin{array}{c}(0.012) \\
0.019^{* * *} \\
(0.002)\end{array}$ & $\begin{array}{c}(0.015) \\
0.0222^{* * *} \\
(0.003)\end{array}$ \\
\hline Current salary of & $-0.025^{* * *}$ & $-0.094^{* * *}$ & $-0.051^{* * *}$ & $-0.021^{* * *}$ & $-0.024^{* * *}$ \\
\hline
\end{tabular}


person/1000

\begin{tabular}{lccccc} 
& $(0.004)$ & $(0.006)$ & $(0.005)$ & $(0.004)$ & $(0.004)$ \\
\multicolumn{1}{c}{ Squared form } & $0.003^{* * *}$ & $0.006^{* * *}$ & $0.004^{* * *}$ & $0.003^{* * *}$ & $0.003^{* * *}$ \\
& $(0.000)$ & $(0.000)$ & $(0.000)$ & $(0.000)$ & $(0.000)$ \\
$\begin{array}{l}\text { Average salary } \\
\text { of the }\end{array}$ & $0.087^{* * *}$ & 0.008 & $0.118^{* * *}$ & $0.090^{* * *}$ & $0.104^{* * *}$ \\
partner/1000 & & & & & \\
& $(0.010)$ & $(0.046)$ & $(0.014)$ & $(0.010)$ & $(0.011)$ \\
Squared form & $-0.014^{* *}$ & 0.002 & $-0.023^{* * *}$ & $-0.014^{* *}$ & $-0.016^{* * *}$ \\
& $(0.005)$ & $(0.027)$ & $(0.008)$ & $(0.006)$ & $(0.006)$ \\
Current salary of & $-0.038^{* * *}$ & $-0.030^{* * *}$ & $-0.050^{* * *}$ & $-0.037^{* * *}$ & $-0.043^{* * *}$ \\
the partner/1000 & & & & & \\
& $(0.005)$ & $(0.011)$ & $(0.007)$ & $(0.005)$ & $(0.006)$ \\
\multicolumn{1}{c}{ Squared form } & $0.003^{* *}$ & 0.001 & $0.005^{* * *}$ & 0.002 & 0.002 \\
& $(0.002)$ & $(0.003)$ & $(0.002)$ & $(0.002)$ & $(0.002)$ \\
& & & & & \\
Observations & 56,377 & 56,377 & 56,377 & 56,377 & 56,377 \\
\hline
\end{tabular}

Note: Full sample of person-year observations 2005-2010. Standard errors are in parentheses. For Probit regressions, the table reports marginal effects estimated at the mean. ${ }^{* * *} \mathrm{p}<0.01,{ }^{* *} \mathrm{p}<0.05,{ }^{*} \mathrm{p}<0.1$. 
4.A.5. REGRESSION RESULTS - WOMEN WITH ITAX

\begin{tabular}{|c|c|c|c|c|c|}
\hline & LPM & Fixed effect & $\begin{array}{c}\text { Random } \\
\text { effect }\end{array}$ & Probit & $\begin{array}{l}\text { Probit } \\
\text { random } \\
\text { effects }\end{array}$ \\
\hline Accrual/ 100,000 & $\begin{array}{l}0.096^{* * *} \\
(0.008)\end{array}$ & $\begin{array}{l}0.159^{* * *} \\
(0.011)\end{array}$ & $\begin{array}{l}0.117^{* * *} \\
(0.009)\end{array}$ & $\begin{array}{c}0.075^{* * *} \\
(0.008)\end{array}$ & $\begin{array}{l}0.080^{* * *} \\
(0.008)\end{array}$ \\
\hline SSW/100,000 & $\begin{array}{l}0.256^{* * *} \\
(0.009)\end{array}$ & $\begin{array}{l}-0.060^{* *} \\
(0.025)\end{array}$ & $\begin{array}{l}0.294^{* * *} \\
(0.012)\end{array}$ & $\begin{array}{c}0.247^{* * *} \\
(0.008)\end{array}$ & $\begin{array}{c}0.234^{* * *} \\
(0.011)\end{array}$ \\
\hline Age & $\begin{array}{l}0.494^{* * *} \\
(0.052)\end{array}$ & $\begin{array}{l}0.126^{* *} \\
(0.063)\end{array}$ & $\begin{array}{c}0.181^{* * *} \\
(0.052)\end{array}$ & $\begin{array}{c}0.590^{* * *} \\
(0.049)\end{array}$ & $\begin{array}{c}0.577^{* * * *} \\
(0.051)\end{array}$ \\
\hline Age squared & $\begin{array}{c}-0.004^{* * *} \\
(0.000)\end{array}$ & $\begin{array}{l}-0.001^{*} \\
(0.001)\end{array}$ & $\begin{array}{l}-0.001^{* * *} \\
(0.000)\end{array}$ & $\begin{array}{c}-0.005^{* * *} \\
(0.000)\end{array}$ & $\begin{array}{c}-0.004^{* * *} \\
(0.000)\end{array}$ \\
\hline SEA & $\begin{array}{c}0.255^{* * *} \\
(0.016)\end{array}$ & $\begin{array}{l}0.322^{* * *} \\
(0.019)\end{array}$ & $\begin{array}{c}0.203^{* * *} \\
(0.016)\end{array}$ & $\begin{array}{c}0.269^{* * *} \\
(0.026)\end{array}$ & $\begin{array}{c}0.169^{* * * *} \\
(0.014)\end{array}$ \\
\hline Partner & $\begin{array}{c}0.022^{* * *} \\
(0.006)\end{array}$ & $\begin{array}{c}0.062 \\
(0.049)\end{array}$ & $\begin{array}{c}0.022^{* * *} \\
(0.008)\end{array}$ & $\begin{array}{l}0.020^{* * *} \\
(0.005)\end{array}$ & $\begin{array}{c}0.021^{* * *} \\
(0.006)\end{array}$ \\
\hline Active partner & $\begin{array}{c}-0.027^{* * *} \\
(0.005)\end{array}$ & $\begin{array}{l}-0.014 \\
(0.009)\end{array}$ & $\begin{array}{c}-0.021^{* * *} \\
(0.006)\end{array}$ & $\begin{array}{c}-0.022^{* * *} \\
(0.005)\end{array}$ & $\begin{array}{c}-0.0233^{* * *} \\
(0.005)\end{array}$ \\
\hline Brussels & $\begin{array}{l}-0.012^{*} \\
(0.006)\end{array}$ & & $\begin{array}{l}-0.018^{*} \\
(0.010)\end{array}$ & $\begin{array}{l}-0.011^{*} \\
(0.006)\end{array}$ & $\begin{array}{l}-0.012^{*} \\
(0.006)\end{array}$ \\
\hline Wallonia & $\begin{array}{l}-0.010^{* *} \\
(0.004)\end{array}$ & & $\begin{array}{l}-0.013^{*} \\
(0.007)\end{array}$ & $\begin{array}{l}-0.010^{* *} \\
(0.004)\end{array}$ & $\begin{array}{l}-0.010^{* *} \\
(0.004)\end{array}$ \\
\hline Part-time & $\begin{array}{l}0.007 \\
(0.005)\end{array}$ & $\begin{array}{c}-0.043^{* * *} \\
(0.011)\end{array}$ & $\begin{array}{l}-0.003 \\
(0.006)\end{array}$ & $\begin{array}{l}0.008^{*} \\
(0.004)\end{array}$ & $\begin{array}{l}0.009^{*} \\
(0.004)\end{array}$ \\
\hline White collar & $\begin{array}{l}-0.010^{*} \\
(0.005)\end{array}$ & $\begin{array}{l}-0.050 \\
(0.038)\end{array}$ & $\begin{array}{l}-0.012 \\
(0.008)\end{array}$ & $\begin{array}{l}-0.007 \\
(0.005)\end{array}$ & $\begin{array}{l}-0.007 \\
(0.005)\end{array}$ \\
\hline Primary sector & $\begin{array}{c}0.001 \\
(0.036)\end{array}$ & & $\begin{array}{l}-0.010 \\
(0.056)\end{array}$ & $\begin{array}{c}0.010 \\
(0.038)\end{array}$ & $\begin{array}{c}0.008 \\
(0.038)\end{array}$ \\
\hline Tertiary sector & $\begin{array}{l}-0.008^{*} \\
(0.004)\end{array}$ & & $\begin{array}{c}0.001 \\
(0.006)\end{array}$ & $\begin{array}{c}-0.011^{* * *} \\
(0.004)\end{array}$ & $\begin{array}{c}-0.011^{* * *} \\
(0.004)\end{array}$ \\
\hline $\begin{array}{l}\text { Average salary } \\
\text { of the reference } \\
\text { person/1000 }\end{array}$ & $-0.314^{* * *}$ & $-3.416^{* * *}$ & $-0.343^{* * *}$ & $-0.309^{* * *}$ & $-0.332^{* * *}$ \\
\hline Squared form & $\begin{array}{c}(0.022) \\
0.021^{* * *} \\
(0.005)\end{array}$ & $\begin{array}{c}(0.326) \\
0.464^{* * *} \\
(0.053)\end{array}$ & $\begin{array}{l}(0.031) \\
0.017^{* *} \\
(0.008)\end{array}$ & $\begin{array}{c}(0.021) \\
0.020^{* * *} \\
(0.005)\end{array}$ & $\begin{array}{c}(0.024) \\
0.022^{* * *} \\
(0.006)\end{array}$ \\
\hline $\begin{array}{l}\text { Current salary of } \\
\text { the reference }\end{array}$ & $-0.067^{* * *}$ & $-0.089^{* * *}$ & $-0.092^{* * *}$ & $-0.055^{* * *}$ & $-0.059^{* * *}$ \\
\hline
\end{tabular}




$\begin{array}{lccccc}\text { person/1000 } & & & & \\ & (0.007) & (0.016) & (0.009) & (0.006) & (0.006) \\ \text { Squared form } & 0.013^{* * *} & 0.012^{* * *} & 0.016^{* * *} & 0.011^{* * *} & 0.0011^{* * *} \\ & (0.001) & (0.003) & (0.002) & (0.001) & (0.001) \\ \text { Average salary } & 0.033^{* * *} & 0.080^{* * *} & 0.053^{* * *} & 0.028^{* * *} & 0.029^{* * *} \\ \text { of the } & & & & & \\ \text { partner/1000 } & & & & & \\ & (0.007) & (0.026) & (0.010) & (0.007) & (0.007) \\ \text { Squared form } & -0.003 & -0.017^{*} & -0.007^{*} & -0.003 & -0.003 \\ & (0.003) & (0.009) & (0.004) & (0.003) & (0.026) \\ \text { Current salary of } & -0.015^{* * *} & -0.055^{* * *} & -0.028^{* * *} & -0.011^{* * *} & -0.012^{* * *} \\ \text { the partner/1000 } & & & & & \\ & (0.003) & (0.007) & (0.004) & (0.003) & (0.003) \\ \text { Squared form } & 0.001^{* *} & 0.004^{* * *} & 0.002^{* * *} & 0.001^{* *} & 0.001^{* *} \\ & (0.000) & (0.001) & (0.001) & (0.000) & (0.000)\end{array}$

$\begin{array}{llllll}\text { Observations } & 30,289 & 30,289 & 30,289 & 30,289 & 30,289\end{array}$

Note: Full sample of person-year observations 2005-2010. Standard errors are in parentheses. For Probit regressions, the table reports marginal effects estimated at the mean. ${ }^{* *} \mathrm{p}<0.01,{ }^{* *} \mathrm{p}<0.05,{ }^{*} \mathrm{p}<0.1$. 


\section{WORK, LABOR FORCE EXIT AND BENEFIT CLAIMING PATTERNS IN BELGIUM \\ Anne-Lore Fraikin and Alain Jousten}




\subsection{INTRODUCTION}

In the literature and in the public debate, the transition from employment to inactivity is often summarized into a binary decision: retirement. Said differently, the decision to retire is frequently equaled to simultaneously stop working and claiming (early-) social security benefits. This is the case in numerous studies that analyze the impact of incentives generated by the social security system on the retirement decision (see Diamond and Gruber, 1999; Blondal and Scarpetta, 1999; Dellis et. al., 2004, among others).

Increasingly, however, individuals face more complex choice sets, that sometimes lead them to - optimally or not - disjoin labor market exit from social security benefit claiming. Few papers document the differences between the decisions to exit the labor force and to claim social security benefit. The standard definition of retirement is usually based on the working status of the individual and on the simultaneous receipt of social security benefits. Said differently, an individual is usually defined as retired once he stops working for pay and starts claiming social security benefits. However, MacInnis (2009) reminds that retirement and retirement benefit claiming are two separate decisions that individuals often approach simultaneously. Coile et. al. (2002) illustrate that there exist substantial retirement incentives embedded in the US Social Security system that encourage people to optimally disjoin the labor force exit and benefit claiming decisions. In fact, part of the benefit of continued work on pension wealth is tied to delayed benefit claiming. Therefore, assuming that both decisions are simultaneous overstates the effect

of labor force exit on pension wealth. Gustman and Steinmeier (2015) develop a structural model of benefit claiming, wealth and labor force exit that includes the option to reverse from states of lesser to greater work. 
Research that focuses on a single binary decision yields an incomplete and biased view of how the social security system influences the behavior of older workers for three reasons.

First, social security reforms can potentially affect the decision to exit the labor force and the decision to claim social security benefits differently - possibly disjoining them. Gustman and Steinmeier (2015) and Benítez-Silva and Yin (2009) analyze the impact of social security reforms ${ }^{176}$ and find a differentiated effect of the reforms on full-time work and benefit receipt. In another paper, Gustman and Steinmeier (2004) study the impact of the elimination of an earnings test for work above the early eligibility age and find that the reform increased the employment rate of older workers but resulted in an earlier flow of benefit claiming. Further, they study the effect of an increase in the early eligibility age and find that it increased the employment rate but delayed the flow of benefit claiming. Haben (2010) studies the impact of the introduction of permanent benefit reductions for early retirees and finds that this reform delayed benefit claiming by an average of 14 months and employment exit by an average of 10 months. Staubli and Zweimüller (2013) study the impact of an increase in the early retirement age in Austria and find that it had a positive impact on the employment rate of high wage and healthy workers. However, they also find that it increased unemployment benefit claims of low-wage and unhealthy workers, who tend to use these benefits to bridge the newly created gap to the early retirement age.

Second, the option of combining (part-time) work with social security benefits claiming influences labor market decisions at older ages, arguably encouraging some workers to stay in the labor force longer (Schmid, 1998). For instance, the time credit scheme allows

\footnotetext{
${ }^{176}$ Gustman and Steinmeier (2015) simulate the effect of an increase in the statutory and early eligibility ages of the old-age pension and the removal of the payroll tax after an individual has reached the statutory retirement age. Benítez-Silva and Yin (2009) analyze the impact of an increase in the statutory retirement age, the elimination of an earnings test and an increase in the delayed retirement credit on the benefit claiming decision and on the level of benefit receipt.
} 
for part-time work and part-time unemployment benefit receipt at older ages ${ }^{177}$. Such program was implemented with the objective of keeping older workers on the labor force longer.

On the upside, Duval (2003) notes that because of the decreasing marginal utility of work, part-time work (with or without benefit claiming) can improve the welfare of older workers and be a valuable policy tool to reduce the incentives to leave the labor force early. Moreover, Benítez-Silva and Heiland (2008) use US data and a duration analysis framework and find that the longer a worker who claims early old-age pension benefits stays on the labor force, the less likely he is to exit the labor force before the statutory eligibility age.

On the downside, Albanese et. al. (2015) analyze the impact of the Belgian time credit scheme and find that this program has a positive effect on labor market participation for the first two (four) years of continuous benefit receipt for men (women), after which the opposite effect is observed. Nevertheless, once individuals become eligible for the early old-age pension, they find that the time credit scheme has a negative effect on work. Finally, using empirical data from nine OECD countries (including Belgium), BörschSupan et. al. (2017) find that allowing for the combination of work and partial old-age pension benefits claiming has a small positive impact on total labor force participation but a negative impact on the total number of hours worked in the economy as some workers who would have remained employed full-time until retirement might now opt for part-time work.

Third, distinguishing labor force exit from benefit claiming decisions and identifying both short-and long-run term fiscal costs of benefit claims are two issues of prime policy

\footnotetext{
177 See chapter two for a detailed review of the time credit scheme.
} 
importance in Belgium. The country has a highly complex landscape of labor force exit and benefit claiming options - that are not always fine-tuned. Indeed, the ubiquitous nature of partial or full labor force exit options - even at rather low ages - and the absence of penalty for early claimers of old-age pension benefits renders the short and the longterm fiscal stakes particularly high. From a public finance point of view, periods of social security benefits receipt generate immediate costs through benefit payouts (e.g., unemployment benefits) but may also generate substantial future fiscal costs as most social security benefits are associated with uninterrupted pension accruals combined with an absence of contribution payments ${ }^{178}$. This is in sharp contrast to many other social security systems in Europe and beyond (e.g., Germany and the US) where the strict reliance on a Bismarckian insurance logic ensures that benefit accrual is conditional on contribution payment. ${ }^{179}$

The purpose of this chapter is to document the evidence of the split-up of retirement into two distinct decisions: the exit of the labor force and the claim of social security benefits. Such distinction leads us to consider four different statuses of individuals, based on work and benefit claiming. We demonstrate the relevance of such classification by displaying descriptive evidence of each status using an administrative dataset of the Crossroad Bank for Social Security. Further, we analyze the determinants of both decisions separately and test the assumption that the combination of work and benefit claiming encourages older workers to stay on the labor force longer. The aim of the chapter is to pave the groundwork for more refined future analysis of individual retirement behavior and to raise awareness on potential distinct impact of reforms on both decisions and on fiscal

\footnotetext{
178 See chapter two for more information on the social security programs that allow for continued accruals in pension benefits.

${ }^{179}$ In Germany, as well as in large parts of Central and Eastern Europe, contribution payment by an individual or on his behalf is required for periods of activity or covered inactivity. In the US, benefit accrual is linked to earnings only, simply excluding periods on other types of (replacement) income from pension accrual.
} 
cost considerations of different types of workers. Such analysis will serve to inform and facilitate the policy making process regarding the reforming of the old-age pension system.

The remainder of the chapter is structured as follows. The next section defines a classification of the population into four statuses that allows for the distinction between the labor exit and benefit claiming decisions and summarizes the key characteristics of the dataset. Section 5.3 documents the employment rates of older Belgian workers from 2004 to 2010 and presents empirical evidence of the differences between labor exits and benefit claiming decisions. Section 5.4 introduces the methodology for section 5.5 that contains the analysis of the determinants of labor force exit and benefit claiming. Finally, section 5.6 concludes.

\subsection{KEY CONCEPTS AND DATA}

\subsubsection{LABOR FORCE EXIT AND THE BENEFIT CLAIMING DECISIONS}

In the literature and in the public debate, the transition from work to inactivity at the end of working life is often summarized into a single decision: retirement, which involves both stopping to work and starting to claim some form of social security benefits. While such a view on transitions might have been appropriate in the past, this is not necessarily the case anymore when individuals face complex incentives generated by modern-day social security programs.

The Belgian social security system clearly generates an environment where labor force exit and social security benefit claiming are no longer necessarily synchronized, be it optimally or de facto. There is a wide array of pathways from work into retirement, some of which allow for full or part-time periods of social security benefits claims combinable 
with (continued or new) streams of work income. As a result, four general statuses of individuals emerge: (1) work and no claim, (2) work and claim, (3) no work and claim, (4) no work and no claim.

1. The first status, work and no claim, includes individuals who work (full or parttime) without claiming any type of social security benefits based on their own earnings records. It also comprises workers who receive derived old-age pension benefits, such as the survivor pension or those workers whose spouse receive the old-age pension benefit at the household replacement rate.

2. The second status, work and claim, includes individuals who work (full or parttime) and claim social security benefits based on their own earnings records. ${ }^{180}$ For instance, older workers can claim time credit benefits or receive benefits from temporary or part-time unemployment insurance, part-time conventional early retirement, part-time disability insurance, etc. ${ }^{181}$ Individuals who receive old-age pension benefits can also work and receive an income, which is subject to an earnings limit.

3. The third status, no work and claim, contains individuals who do not work but claim social security benefits based on their own earnings record. These social security benefits include benefits from the old-age pension program but also from programs that serve as early retirement pathways such as the disability insurance, the conventional early retirement and the unemployment insurance.181 Individuals in this

\footnotetext{
${ }^{180}$ Different social security institutes have different registration timing of their variables. For instance, the old-age pension office records the status information at the end of the period while the employment office records the information at the beginning of the period. Therefore, in order to avoid that individuals transferring from a status to another are classified in the status work and claim, we impose a condition of a minimum of two years in the status work and claim. In the case an individual is identified for only one year in the status work and claim, we transform the observation into the destination status. For example, if an individual is labelled as working (year 1) - working and receiving disability benefits (year 2) - receiving disability benefits (year 3), then we relabel the second year as receiving disability benefits only.

${ }^{181}$ Older workers can also receive (part-time) social assistance benefits, (part-time) work injury or (part-time) professional disease benefits but these programs play a less prominent role in the retirement analysis debate.
} 
status can combine social security benefits based on their own earnings records with derived old-age pension benefits (survivor pension for instance).

4. The fourth and last status, no work and no claim, includes people who neither work nor claim any benefits based on their own earnings records. Such individuals can either be financially dependent on their spouse or benefit from derived old-age pension benefits such as the survivor pension or have a spouse who receives old-age pension benefits at the household replacement rate.

From these statuses, we define the labor force exit and the benefit claiming decisions (see figure 5.1). The labor force exit decision can be interpreted as a transfer from either of the two work statuses (work and no claim or work and claim), to either of the two non-work statuses (no work and claim or no work and no claim). The social security benefit claiming decision can be interpreted as a transfer from either of the two no claim statuses (work and no claim or no work and no claim), to either of the two claim statuses (work and claim or no work and claim).

Figure 5.1: Classification of individuals according to their work and claim status and the labor force exit and benefit claiming decisions

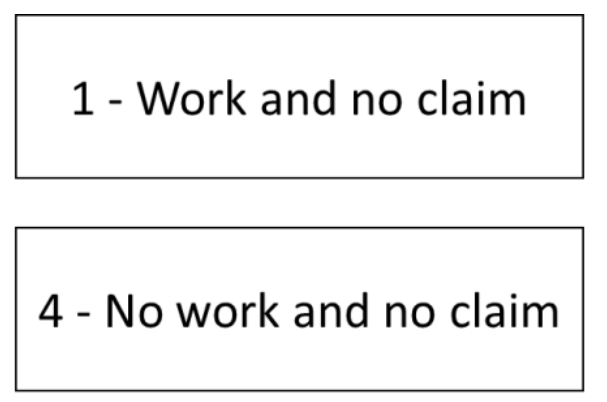

No claim

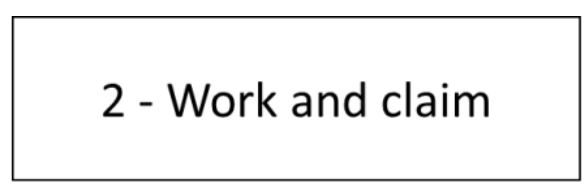

\section{3 - No work and claim}
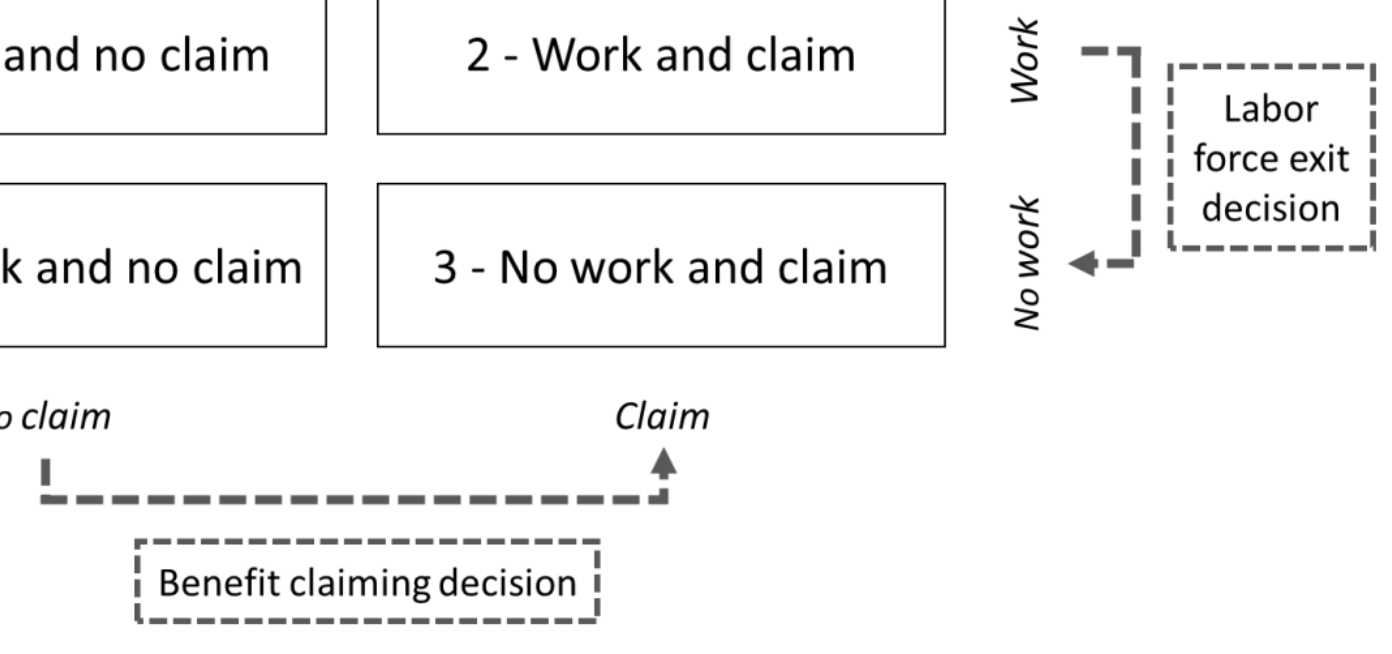

Source: Authors' own realization 
When introducing this classification, the idea is clearly not a pure accounting one. This type of grouping of individuals matters for real world policy problems. First, for researchers and policymakers interested in labor markets, it is key to know the characteristics of workers to gauge how they are influenced by different retirement options and to predict how labor might respond to policy changes. For example, when considering policies targeting a reduced tax wedge on labor, it is essential to understand what type of jobs are being created: are these new pure full-time jobs, or are they parttime jobs that allow for the claim of social security benefits. If the latter, the beneficial effect of extra workers is likely somewhat lower than in the first case and any possible self-financing effects of labor tax wedge reforms might be severely hampered. ${ }^{182}$

Second, for researchers interested in the long-term financial sustainability of the pension system or the broader social security system, the distinction between labor force exit and benefit claiming also matters. For example, while partial pension arrangements might induce some people to work longer (see Benítez-Silva and Heiland, 2008), they may also have large fiscal costs if benefits are not adjusted in (at least) an actuarially fair way. Indeed, in Belgium, benefit claiming is often not only associated with current fiscal costs but also with differed fiscal liabilities, as periods spent on benefits count towards the pension history, be it at the rate of the last wage or a lower lump-sum amount. In fact, differed fiscal costs can easily outweigh current costs because of non-linear accrual of future pension entitlements. ${ }^{183}$

\footnotetext{
182 The analysis of Capéau et al (2018) on the self-financing nature of recent labor tax reforms in Belgium could be further strengthened by our classification.

${ }^{183}$ For example, as a result of pension accrual for periods of inactivity, individuals may pass some hurdles in terms of minimum pension eligibility.
} 


\subsubsection{DATA AND SAMPLE SELECTION}

We use a detailed administrative panel dataset retrieved from the Datawarehouse Labor Market and Social Protection of the Belgian Crossroad Bank for Social Security that combines data from various social security institutions and the national registry. ${ }^{184}$ The dataset initially contains quarterly information for the years 2004 to 2010 on the labor market status, social security benefit receipt status and personal characteristics of 150,000 individuals aged between 51 and 63 in 2004. The dataset also contains detailed information on the earnings and social security benefit receipt histories. Finally, the dataset includes similar information for each partner ${ }^{185}$ of the sampled individuals, if applicable.

From these initial cohorts, we select the observations of individuals aged between 55 and 64 between 2004 and 2010 and we drop the observations of individuals after they die. We collapse the data into one single observation per year and per individual, using the information of the last quarter of each year. Similarly to previous chapters, we focus our attention on individuals in the wage-earner regime since they represent the majority of workers in Belgium and because of the sheer complexity and differences between the different working regimes. ${ }^{186}$ Therefore, we select only individuals who were working (full or part-time) as a wage earner ${ }^{187}$ in the first year of observation (year 2004 or age 55 for those who were younger than 55 in 2004), regardless of their benefit claiming status. Further, we add the condition of a total minimum wage-earner career of 10 years (of at

\footnotetext{
184 This dataset was initially created for the EMPOV project (Employment and Poverty in a Changing Society), financed by the Belgian Science Policy Administration (BELSPO research project TA/00/45)

185 Married or legally cohabitating.

${ }^{186}$ See chapter two for a thorough explanation of why we focus on wage earner workers.

187 Individuals with mixed careers, i.e. that have been affiliated with the civil servant or the self-employed regime in addition to their wage earner status are also included.
} 
least one third of a full-time occupation ${ }^{188}$ each). Finally, we discard the observations of workers who transfer to the self-employed or civil-servant working regime before they retire or before the end of our observation period.

Our final sample is composed of 27,666 individuals and 146,522 observations for years ranging from 2004 to 2010 and ages ranging from 54 to $64 .{ }^{189}$ In Table 5.1, we report key personal characteristics of our sampled individuals as they appear in the first year of observation. Unsurprisingly, our sample is composed of mostly males because of the career condition we impose on our sample.

Table 5.1: Main characteristics of sampled individuals in the first year of observation

\begin{tabular}{cccc} 
& & Number of observations & Proportion of total sample \\
\hline \multirow{2}{*}{ Sex } & Male & 10,215 & $36.92 \%$ \\
& Female & 17,451 & $63.08 \%$ \\
\hline \multirow{3}{*}{ Region } & Flanders & 16,121 & $58.247 \%$ \\
& Wallonia & 9,202 & $33.26 \%$ \\
& Brussels & 2,340 & $8.47 \%$ \\
\hline \multirow{2}{*}{ Marital status } & Married & 19,987 & $72.24 \%$ \\
& Unmarried & 7,679 & $27.76 \%$ \\
\hline
\end{tabular}

Source: CBSS dataset

\footnotetext{
${ }^{188}$ This corresponds to at least 104 working or assimilated days (ie. days spent on replacement income) in a year, which is the condition for a year of career to be taken into account in the early eligibility criteria of the old-age pension benefit.

${ }^{189}$ Because of the structure of the data, we do not have data on each age-year combination. We are missing information on individuals aged 55 in 2009 and 2010, aged 56 in 2010, 64 and 65 in 2004 and 65 in 2005. Thus, in section 5.3, we keep the number of observations constant for these missing cases, based on the closest year observable of the same age. We do this in order to present figures that are formed of the same number of cohorts for each year and age.
} 


\subsection{DESCRIPTIVE ANALYSIS OF EMPLOYMENT AND RETIREMENT BEHAVIOR}

In this section, we first present a description analysis of the employment rates of older Belgian workers using our classification method that separates workers based on the benefit claiming status. Then, we document the retirement behavior of older Belgian workers before looking at the labor force exit and benefit claiming hazard rates separately.

\subsubsection{EMPLOYMENT AND BENEFIT CLAIMING}

The Belgian labor market has undergone profound changes over the last three decades. The employment rate of older men was declining until the mid-1990's, after which it started to rise again, most importantly for the 55 to 59 age group. The employment rate of older women follows a long-term upward trend and has been growing at a faster pace than the employment rate of men. In line with Jousten and Lefebvre (2016), we find a general increasing trend of the employment rate between 2004 and 2010 for both sexes and age groups (55 to 59 and 60 to 64 ). Accordingly, we observe a decrease in the proportion of older individuals who are out of the labor force over the same period.

In figure 5.2, we present the employment rates of older workers by age and its decomposition into our two groups differentiated by the benefits claiming status: work and no claim and work and claim. Predictably, the proportion of workers decreases continuously with age - particularly at age 58 and 60, mostly because of transfers to the conventional early retirement program at age 58 and to the conventional early retirement or old-age pension programs at age 60 . Overall, we observe that about one-fourth of workers aged 55 to 64 claim some type of social security benefits: 28.8 (resp. 31.1) percent of working men (resp. women) aged 55 to 59 and 18.3 (resp. 19) percent of working men 
(resp. women) aged 60 to 64 are benefits claimers. These benefit claims mostly include time credit, unemployment and disability benefits.

Figure 5.2: Decomposition of employment rates by claiming status and age (men and women, period average 2004-2010)

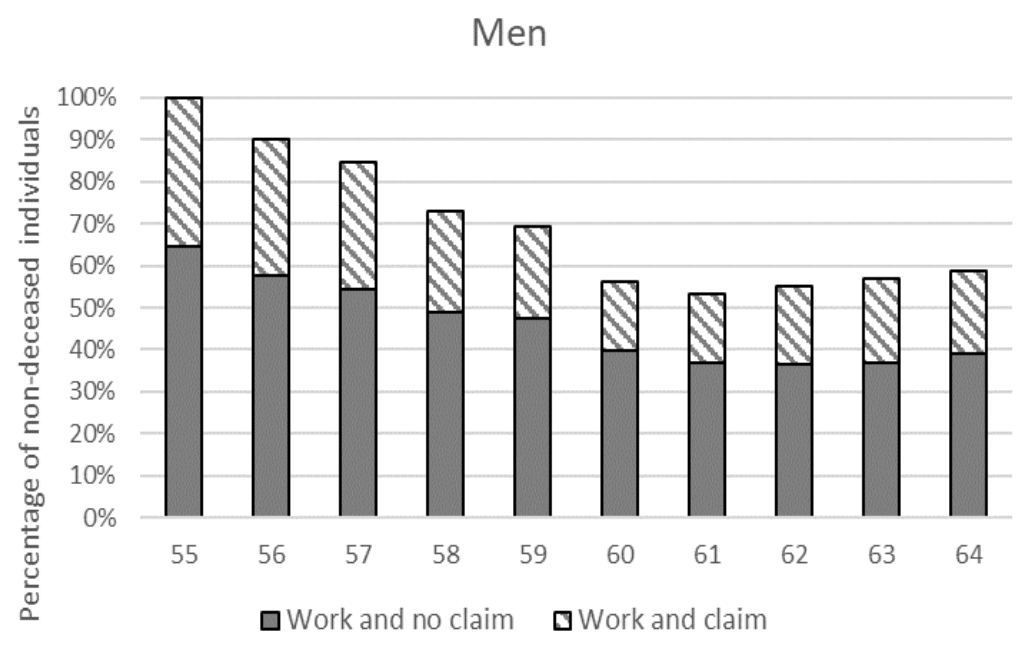

Women

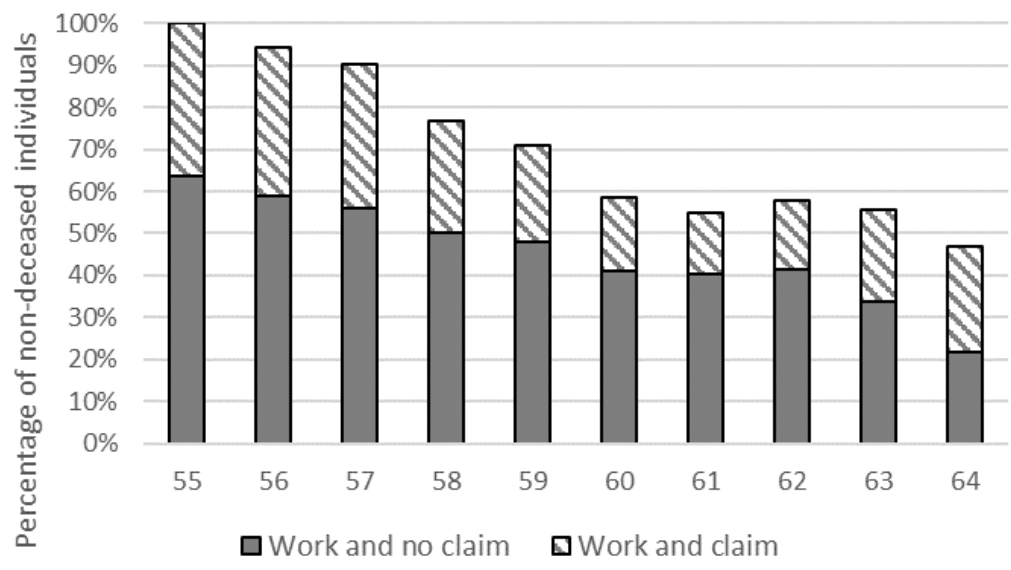

Source: Authors' own calculations using CBSS dataset

In figure 5.3, we decompose our two work groups, differentiated by benefit claiming status, by work intensity (part and full-time work) and by age. We find that part-time work at older ages is frequent in Belgium, particularly for women. In total, 68.7 (resp. 
23.3) percent of working women (resp. men) aged 55 to 59 and 67.2 (resp. 25.8) percent of working women (resp. men) aged 60 to 64 work part-time, regardless of their claiming status. Furthermore, we observe that more than one-third of older workers work parttime. Indeed, 30 (resp. 37.5) percent of men (resp. women) in the group work and no claim and 38.4 (resp. 56) percent of men (resp. women) in the group work and claim work for less than 51 percent of the equivalent of a full-time job. Our results are concomitant with the work of the OECD (2017) that raises Belgium well above the OECD average of 21 percent of part-time work for individuals between the ages of 55 and $64 .{ }^{190}$ Using labor force survey data, Jousten and Lefebvre (2016) find similar results for women, and attribute it partly to a pre-existing larger stock of part-time workers at younger ages hence less an issue of increased transitions from full to part time employment later in life rather than the legacy of previously taken decisions. While male part-timers are mostly claimers, female part-timers are evenly distributed in both claim categories. Finally, we observe that the share of workers (full and part-timers) who do not claim benefits decreases with age, while the share of part-time workers who claim benefits increases with age.

\footnotetext{
${ }^{190}$ In the OECD, only the Netherlands and Switzerland have higher proportions of part-time work at older ages (OECD, 2017).
} 
Figure 5.3: Decomposition of employment rates by claiming status, work intensity and age (men and women, period average 2004-2010)
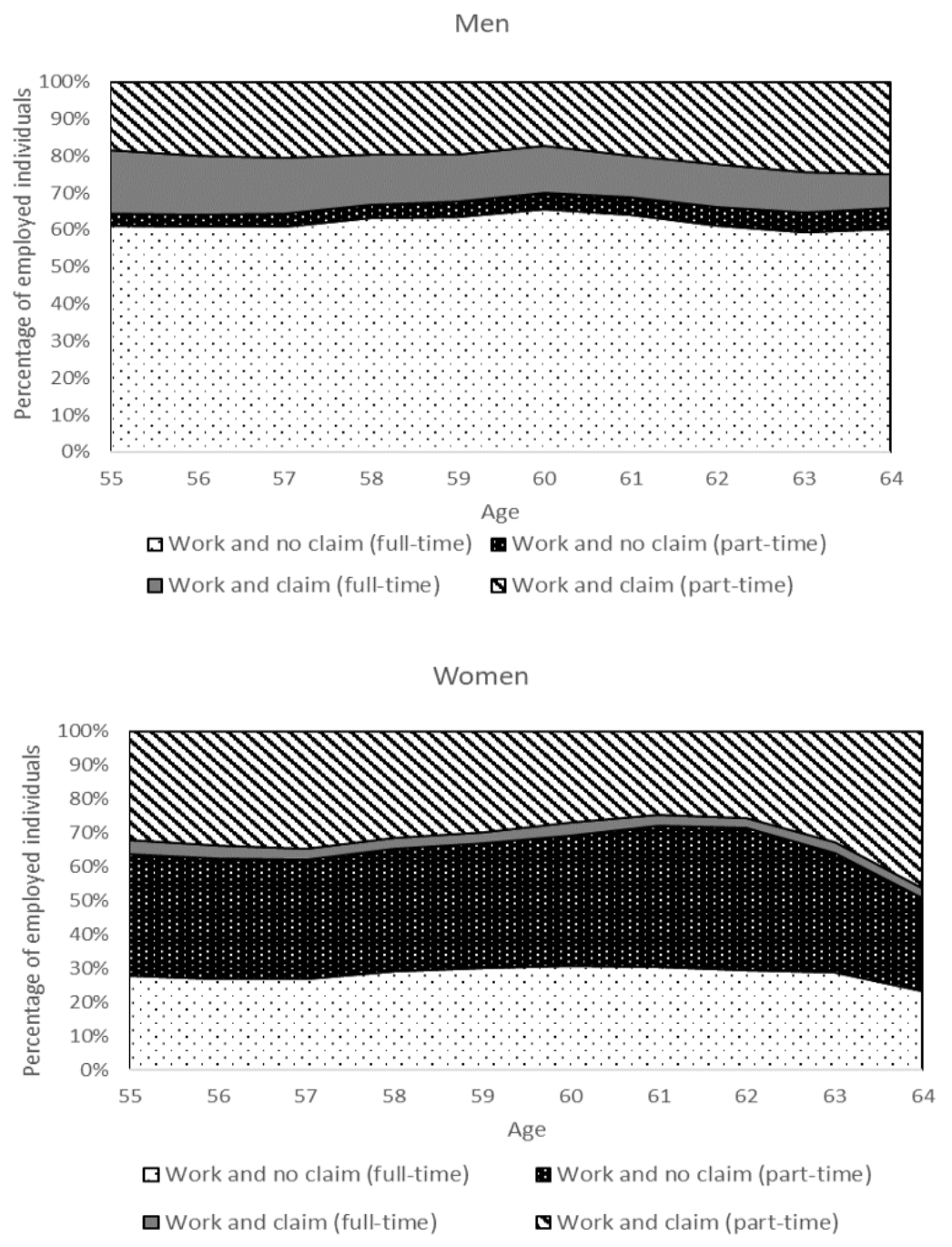

Source: Authors' own calculations using CBSS dataset

In the next figure, we decompose the claims of workers in the group work and claim into five main claims: time credit, disability, unemployment, old-age pension, and other claims. ${ }^{191}$ We find that part-time workers mostly claim time credit and old-age pension benefits (above age 60) while full-time workers mostly claim (temporary) unemployment

${ }^{191}$ Other claims include part-time conventional early retirement benefits, social assistance benefits, professional injury or disease benefits, etc. These claims are far smaller in terms of the number of claimers. 
benefits. Overall, we find that there is a higher proportion of women who claim time credit benefits compared to men, a higher proportion of men who combine work and unemployment benefits compared to women and a similar proportion of men and women who work and claim disability benefits. Finally, we find that men are more likely to combine work and old-age pension benefits than women, which coincides with the results of the OECD (2017).

Figure 5.4: Decomposition of claims in work and claim, by sex and work intensity
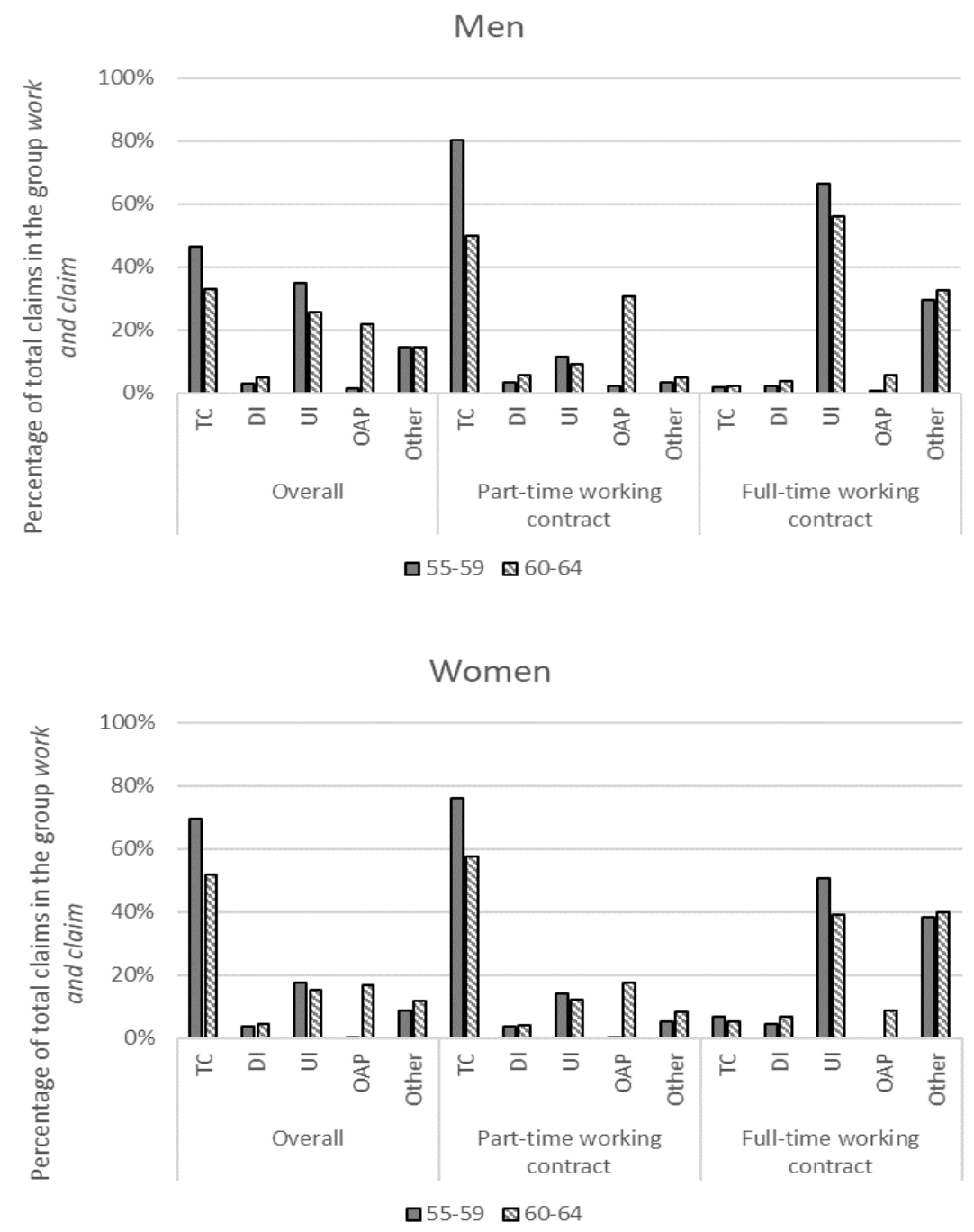

Source: Authors' own calculations using CBSS dataset.

Note: TC stands for time credit, DI stands for disability insurance, UI stands for unemployment insurance, OAP stands for old-age pension. 
Between 2004 and 2010, we note an increase in the share of part-timers in the group work and claim for both men and women aged 55 to 59 , caused by a rise in time credit claims. We also observe an increase in the share of part-timers in the group work and claim for women aged more than 59, mostly caused by a rise in disability benefits claims. Finally, and most importantly, we note a faster increase in the proportion of workers in the group work and claim (except for women above age 59) compared to the proportion of workers in the group work and no claim.

In conclusion, the decomposition of work patterns into our different groups paints a less favorable picture than the mere aggregate employment trends that show an encouraging increasing trend in elderly employment in the last few years : not only have employment levels changed but also the types of employment. Thus, our results coincide with the findings of Aliaj et. al. (2016) that total employment in Belgium has increased over time but the average number of work hours per worker has decreased following a rise in parttime work arrangements. In our case, full-time workers who do not claim any social security benefits only make up approximately 61 percent of total employment of men aged 55 to 59 and 62.7 percent of total employment of men aged 60 to 64. Most importantly, the same proportion drops to approximately 27.6 percent for employed women aged 55 to 59 and to 29.4 percent for employed women aged 60 to 64 .

\subsubsection{LABOR FORCE EXIT AND BENEFIT CLAIMING HAZARD RATES}

In this section, we present the labor force exit and the benefit claiming hazard rates of older Belgian workers. We define the labor force exit hazard rate as the probability that a 
worker (regardless of his benefit claiming status) exits the labor force at a given age. ${ }^{192} \mathrm{We}$ find an average labor force exit age of 59 for men and 59.2 for women. ${ }^{193}$

We define the benefit claiming hazard rate as the probability that an individual (regardless of his working status) starts claiming social security benefits at a given age. ${ }^{194}$ We find an average benefit claiming age of 59.2 for men and 59.3 for women. ${ }^{193}$

Figure 5.5 presents the labor force exit and the benefit claiming hazard rates by age for men and women. We observe a general increase in both the labor force exit and the benefit claiming hazard rates over the age and two major peaks at age 58 (the early eligibility age of the conventional early retirement) and 60 (the early eligibility age of the old-age pension). There is an additional increase in both hazard rates for women starting at age 63 caused by the 1997 reform that raised their statutory eligibility age of the old-age pension from 60 in 1997 to 65 in $2009 .{ }^{195}$ We observe that the labor force exits and the benefit claiming hazard rates of women are generally lower than those of men, which is again caused by our sample selection method. For both men and women, we observe that the labor force exit hazard rate is slightly higher compared to the benefit claiming hazard rate above age 60, caused by a higher prevalence of transfers from the group work and no claim to the group work and claim compared to labor force exits.

\footnotetext{
192 The labor force exit hazard rate is calculated as the number of labor force exits at a certain age over the employed population (statuses work and no claim and work and claim) at end of the precedent age.

193 The relatively high average labor force exit and benefit claiming ages of women compared to men is plausibly caused by our sample selection method according to which we select working women who count at least 10 years of career as a wage-earner and who were still employed at an older age, which is not representative of the actual population of working women in Belgium.

${ }^{194}$ The benefit claiming hazard rate is calculated as the number of individuals who start claiming social security benefits at a certain age over the non-benefit claiming population (statuses work and no claim and no work and no claim) at the end of the precedent age.

195 This reform has raised the retiring peak from 63 to 64 between 2006 and 2008 and from 64 to 65 starting from 2009. The same peak in labor force exit and claiming hazard rates would be observed at men's statutory eligibility age (65), were it part of our observed ages.
} 
If we look at the evolution of the hazard rates over time, we observe that the labor force exit hazard rate has decreased between 2004 and 2010 in both age groups and for both sexes, regardless of the initial claiming status (not graphically represented). Such a trend is caused by a lower number of transfers from the group work and no claim to the group no work and claim caused by a decrease in transfers to the conventional early retirement (before age 60), the unemployment programs (before age 60) and the old-age pension (after age 59). ${ }^{196}$ The benefit claiming hazard rate also displays a decreasing trend over the period 2004 to 2010 for workers aged 60 to 64 but it is increasing for workers aged 56 to 59. The increase in benefit claiming hazard rate before age 60 is caused by a higher number of transfers from the status work and no claim to work and claim (caused by the time credit and part-time unemployment claims) compared to transfers from the groups work and no claim to no work and claim.

\footnotetext{
${ }^{196}$ We also observe a slight increase in the transfers to the conventional early retirement after age 59, which is not high enough to absorb the decrease in transfers to the old-age pension.
} 
Figure 5.5: Labor force exit and benefit claiming hazard rates by age and sex (period
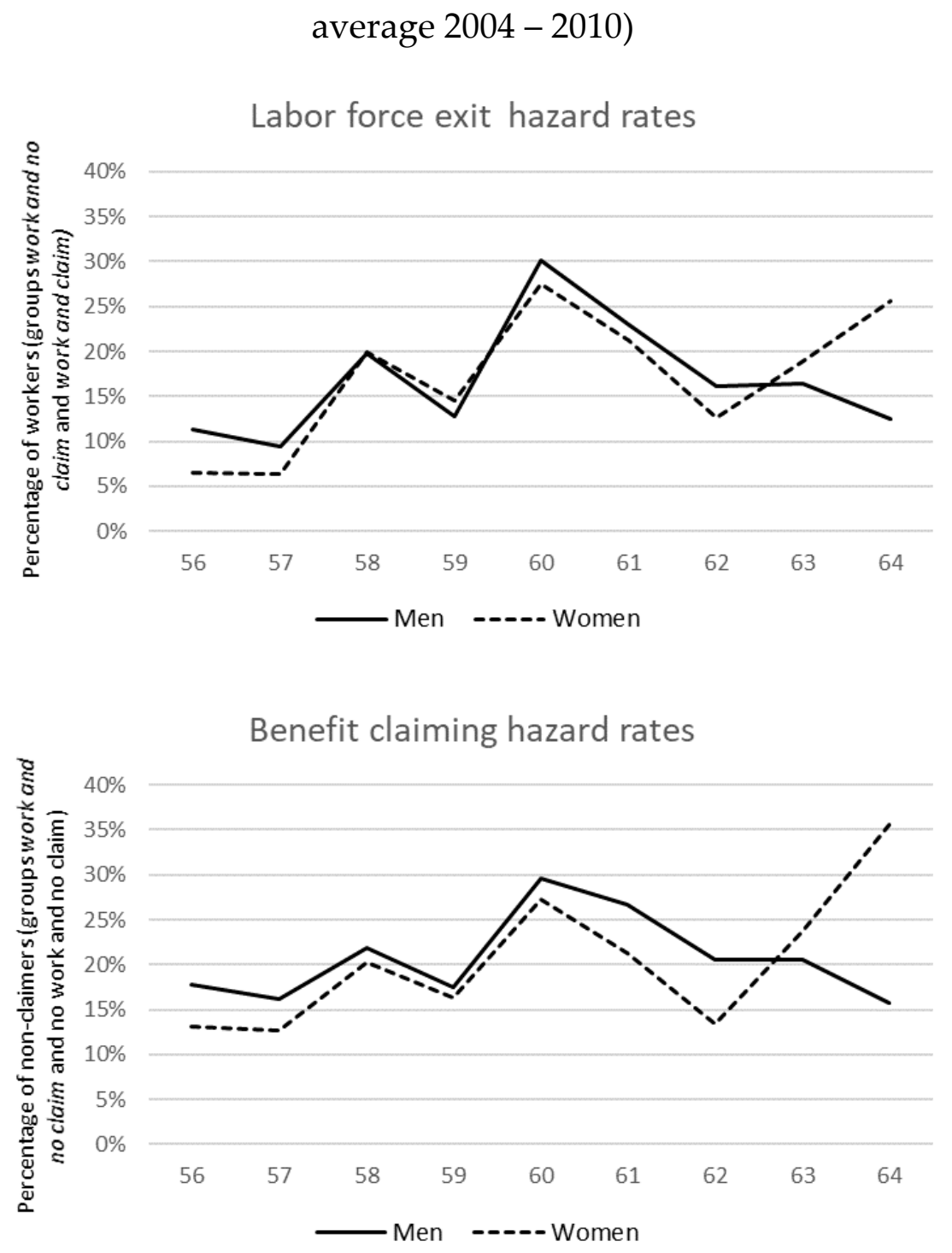

Source: Authors' own calculations using CBSS dataset

In Figure 5.6, we present the decomposition of the labor force exit hazard rate into its two main components: the proportion of transfers from (i) work and no claim to no work and claim and (ii) work and claim to no work and claim. ${ }^{197}$ First, we observe that the proportion of

197 The proportion of transfer from the status work and (no) claim to the status no work and claim is calculated as the number of exits to the status no work and claim over the number of individuals in the status work and (no) claim at the precedent age. Transfers to the status no work and no claim remain minor and are mostly confined to women. 
labor force exit of workers who are claimers is higher than the proportion of labor force exit of workers who are not claimers at every age and for both sexes (except at age 64 for women). In particular, workers who are claimers are more likely to exit the labor force through the conventional early retirement, the unemployment insurance or to the old-age pension (after age 60 only) systems. Second, we observe that the difference of labor force exit hazard rates between our two claiming groups decreases with age for men.

Figure 5.6: Decomposition of labor force exit hazard rates by claiming status, age and sex (period average 2004-2010)
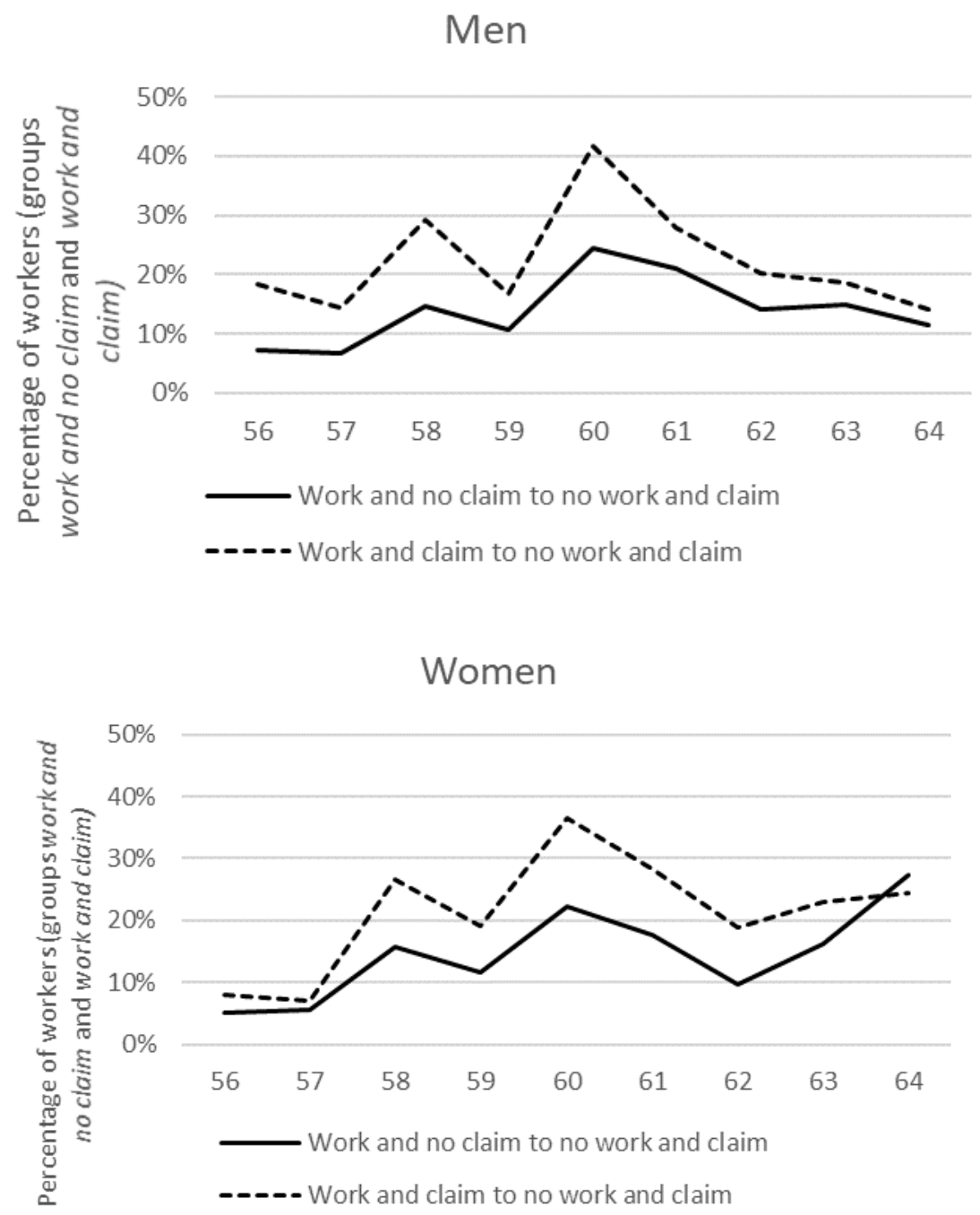

Source: Authors' own calculations using CBSS dataset 
In figure 5.7, we present the decomposition of the benefit claiming hazard rate into its two main components: transfers from the statuses (i) work and no claim to no work and claim and (ii) work and no claim to work and claim. ${ }^{198}$ The proportion of transfer from status work and no claim to status work and claim fluctuates around 7.4 percent for men and 6.3 percent for women and decreases over the age for both sexes (except women aged 63 and above). Moreover, these transfers do not display the same peaks as the labor force exit hazard rate and are mostly caused by time credit and unemployment insurance claims.

\footnotetext{
198 The proportion of transfer from the status work and no claim to the status (no) work and claim is calculated as the number of new claims in the (no) work and claim status from the work and no claim status over the number of individuals in the status work and no claim at the precedent age. Labor force exits to the status no work and no claim remain minor and are mostly confined to women. See above for an explanation of the transfers from the group work and no claim to the group no work and claim.
} 
Figure 5.7: Decomposition of benefit claiming hazard rates by working status, age and sex (period average $2004-2010$ )
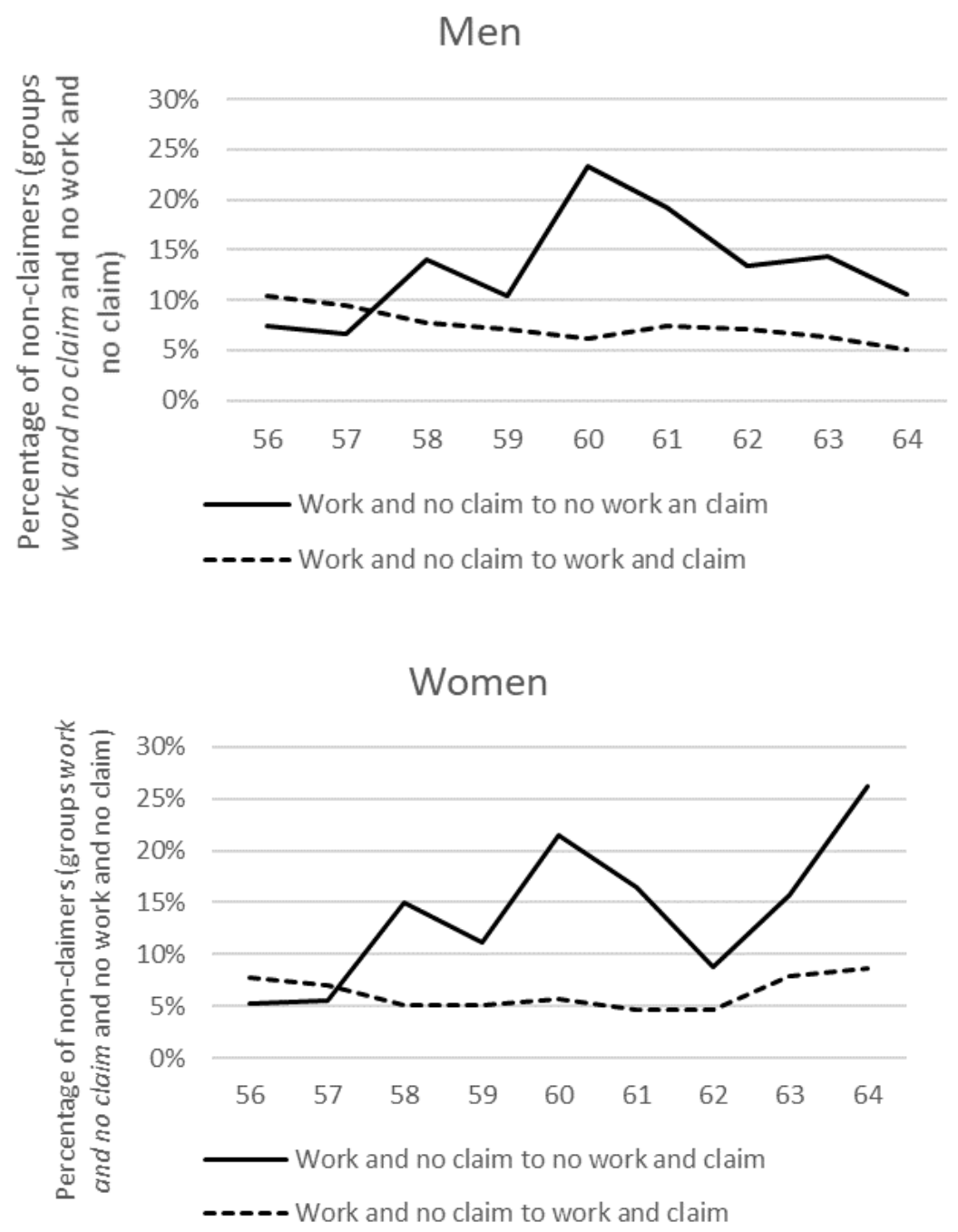

Source: Authors' own calculations using CBSS dataset

\subsection{EMPIRICAL ANALYSIS}

The previous section illustrated the relevance of separating workers by their benefit claiming status and to distinguish between the decisions of labor force exit and (early-) retirement benefit claiming. We now look at the determinants of each decision separately. We use an econometrics model to study the link between our dependent variable and a series of explanatory variables that we define below. 


\subsubsection{VARIABLE DEFINITION AND SUBSAMPLES}

We use three models, each with different dependent variables, one for the labor force exit decision and the other two for the benefit claiming decision. Each individual is observed for 1 to 7 periods between 2004 and 2010. ${ }^{199}$

The labor force exit dependent variable takes a value of 1 in period $t$ if an individual transfers from either of the two work statuses (work and no claim or work and claim) in period $(\mathrm{t}-1)$ to either of the two non-work statuses (no work and claim or no work and no claim) in period t. ${ }^{200}$ For this analysis, we use a subsample that includes individuals in the group work and no claim or work and claim in period $\mathrm{t}$. Following the previous literature (see Dellis et. al. (2004) and Jousten and Tarantchenko (2014)), we consider the decision to exit the labor force as absorbing. Therefore, the observations of individuals are dropped after they exit the labor force. ${ }^{201}$ We obtain a total of 88,217 observations for 27,351 individuals.

$$
\begin{aligned}
& \text { Labor force exit }{ }_{i t} \\
& \qquad\left\{\begin{array}{r}
1 \text { if work status in period }(t-1) \text { and non }- \text { work status in period } t \\
0 \text { otherwise }
\end{array}\right. \\
& t=2, \ldots, 7
\end{aligned}
$$

The first benefit claiming dependent variable takes a value of 1 if an individual transfers from the status work and no claim in period (t-1) to either of the two claim statuses (work

\footnotetext{
199 The number of observation periods per individual depends on the cohort they belong to and on whether they retire or die during our observation period.

${ }^{200}$ Because of the dynamic configuration of our dependent variables, the baseline period $t=1$, which corresponds to year 2004 or age 55, is not included in the analysis.

${ }^{201}$ Additionally, we do not include individuals who exit and reenter the labor force during our observation period as they only represent a minor part of the initial sample.
} 
and claim or no work and claim) in period t. ${ }^{202}$ The second benefit claiming dependent variable takes a value of 1 if an individual transfers from the group work and no claim in period ( $\mathrm{t}-1)$ to the group work and claim in period t. For this analysis, we use a subsample of individuals who are in the group work and no claim in period t. We drop the observations of individuals after they exit the labor force. We allow individuals to claim social security benefits and to revert to a non-claimer status as it occurs often in our data. ${ }^{203}$ We obtain a total of 59,998 observations for 19,808 individuals for the model using the first benefit claiming dependent variable and 52,560 observations for 17,944 individuals for the model using the second benefit claiming dependent variable. ${ }^{204}$

$$
\begin{aligned}
& \text { Claiming }_{\text {it }} \\
& =\left\{\begin{array}{c}
1 \text { if work and no claim status in period }(t-1) \text { and claim status in period } t \\
0 \text { otherwise }
\end{array}\right.
\end{aligned}
$$

$$
\begin{aligned}
& \text { Claiming in work and claim } \text { it } \\
& =\left\{\begin{array}{c}
1 \text { if work and no claim status in period }(t-1) \text { and work and claim status } \\
\text { in period } t \\
0 \text { otherwise }
\end{array}\right.
\end{aligned}
$$

We control for personal characteristics such as gender ( 1 if male), age (using a full set of binary variables to capture age-based focal points see Seibold (2017)), marital status (1 if married) and the region where the individual lives (Wallonia, Brussels or Flanders). We add partner characteristics such as the partners' 205 employment status ( 1 if currently

\footnotetext{
202 We leave aside transfers from the status no work and no claim because of its relatively small size. Moreover, we only observe a very low number of transfers to and from this status. See chapter six for the analysis of the retirement incentives faced by individuals in one-earner households.

${ }^{203}$ For instance, an individual might be considered as incapacitated following health concerns for a year and then start working again. We cannot differentiate between longer-term claims that are related to a retirement strategy and shorter-term claims.

204 The difference of subsample between the two dependent variables comes from the fact that we exclude the claims in the group no work and claim when using the second benefit claiming dependent variable. 205 Partners include married couples and legal cohabitants.
} 
employed) and the partner's social security claiming status (1 if claiming benefits). We add an interaction term between the employment and the claiming status of the partner, indicating if the partner is in the group work and claim. These partner characteristics variables are set to zero if the individual does not have a partner.

We add job related characteristics variables as they were observed at the end of the previous observation period (t-1). ${ }^{206}$ We include the work category ( 1 if the worker is white collar, 0 if the worker is blue collar), the sector of activity of the worker (primary, secondary or tertiary), the sum of lifetime earnings that enter the old-age pension benefit calculation expressed in 100,000 euros (and its squared form) ) $^{207}$ and the average number of assimilated days (days spent on unemployment, disability, etc.) per year during the individual's wage-earner career. We add a variable that indicates the intensity of working hours: full time (reference category), part-time of less than or equal to $50 \%$ of a full-time equivalent (FTE) and part-time of more than $50 \%$ of a FTE.

In the labor force exit model, we use two model specifications differentiated by the benefit claiming indicator. In the first specification, we add a benefit claiming variable (1 if the individual is claiming benefits ${ }^{208}$ ) and we interact it with the working intensity variable defined above. In a second model specification, we split the benefit claiming variable and distinguish between old-age pension, unemployment insurance, disability insurance, time credit and other claims ${ }^{209}$.

\footnotetext{
206 Since our dependent variables indicate a status modification between the end of the precedent period $(t-1)$ and the end of the current period $t$, we include the information at the end of period $(t-1)$ as an indicator of the job characteristics at the beginning of period $t$, which supposedly influences a transfer in period $t$.

${ }^{207}$ Unlike other job characteristics variables, we use the lifetime earnings at the end of period $t$.

${ }^{208}$ This variable differs from the benefit claiming dependent variable: the benefit claiming explanatory variable indicates whether the individual is currently claiming benefits and the benefit claiming dependent variable indicates a transfer from a non-claim status to a claim status.

209 The group "other" captures all of the other claims in the group work and claim that are not time credit, unemployment, disability or old-age pension benefits. Conventional early retirement claims are included
} 
Following the findings of Jousten and Tarantchenko (2014) that program eligibility plays a major role in the retirement decision, we add eligibility variables for the early and the statutory eligibility age of the old-age pension system. ${ }^{210}$ Following our findings in chapter four, which indicate that the pension bonus does not lead to later retirement, we add an eligibility variable for the old-age pension bonus. ${ }^{211}$

Finally, we add a series of variables indicating the year (2005 to 2010) in order to control for any secular changes in the legislation not directly modeled and to avoid spurious regression.

We anticipate an important omitted variable bias in our model because of a lack of data on the health status and wealth (other than earnings-related) of individuals. However, we follow Albanese et. al. (2015) and add an indicator of the average number of days receiving a replacement income for a year over the worker's entire career since we expect health problems and unobservable variables such as ability and motivation to work to be reflected by these career gaps. Furthermore, we expect unobservable characteristics such as productivity and preferences for leisure to be correlated with both the labor force exit and the benefit claiming decision. Indeed, Benítez-Silva and Heiland (2008) expect workers who are more productive to have a lower probability to exit the labor force and to claim benefits later compared to less productive workers. Additionally, individuals

\footnotetext{
in the other category because there are a very low number of conventional early retirement claims in the group work and claim. The combination of disability claims and work is in theory not allowed but is possible in certain situations (see the National Disability Office website at https://www.inami.fgov.be/fr/themes/incapacite-travail/salaries-chomeurs/Pages/reprendre-travailadapte-incapacite-travail.aspx)

210 The early eligibility age of the old-age pension is set at 60 with at least 35 years of career as wage-earner for men and women between 2005 and 2010. The statutory eligibility age is set at 65 for men and 63 for women before 2006, 64 for women between 2006 and 2008 and 65 for women from 2009 onwards. See chapter two for more detailed information on the statutory and early eligibility ages of the old-age pension.

${ }^{211}$ An individual is eligible for the pension bonus if he is aged more than 62 or has more than 44 years of career after 2007. See chapter two for more information on the pension bonus.
} 
who value leisure more have a higher probability to exit the labor force and to claim benefits early than individuals who value leisure less. We control for the effect of these unobservable variables using a correlated random effects model that we present in the appendix since the use of such a model does not alter our results significantly.

Table 5.2 presents the mean values of our three dependent variables and independent variables differentiated by sex. We present the mean values separately for our two subsamples: the sample for the analysis of the labor force exit decision (groups work and no claim and work and claim) and the sample for the analysis of the benefit claiming decisions (group work and no claim). 
Table 5.2: Mean value of dependent and independent variables by status and sex

\begin{tabular}{|c|c|c|c|c|c|c|}
\hline & \multicolumn{3}{|c|}{$\begin{array}{l}\text { Groups work and no claim } \\
\text { and work and claim }\end{array}$} & \multicolumn{3}{|c|}{ Group work and no claim } \\
\hline & Overall & Men & Women & Overall & Men & Women \\
\hline Labor force exit & 0.15 & 0.16 & 0.14 & & & \\
\hline Benefit claim & 0.14 & 0.15 & 0.12 & 0.21 & 0.22 & 0.19 \\
\hline $\begin{array}{l}\text { Benefit claim in the group work } \\
\text { and claim }\end{array}$ & 0.06 & 0.06 & 0.05 & 0.09 & 0.09 & 0.07 \\
\hline Full time contract & 0.60 & 0.77 & 0.32 & 0.75 & 0.94 & 0.44 \\
\hline Part-time contract $(\leq 50 \%$ of FTE) & 0.18 & 0.09 & 0.32 & 0.09 & 0.02 & 0.22 \\
\hline Part-time contract ( $>50 \%$ of FTE) & 0.22 & 0.14 & 0.36 & 0.15 & 0.04 & 0.34 \\
\hline Claiming & 0.34 & 0.33 & 0.35 & 0.03 & 1 & 0 \\
\hline Time credit & 0.18 & 0.15 & 0.23 & & & \\
\hline Unemployment & 0.09 & 0.11 & 0.06 & & & \\
\hline Disability & 0.01 & 0.01 & 0.01 & & & \\
\hline Old-age pension & 0.01 & 0.01 & 0.01 & & & \\
\hline Other & 0.04 & 0.05 & 0.03 & & & \\
\hline $\begin{array}{l}\text { Working and claiming ( } \leq 50 \% \text { of } \\
\text { FTE) }\end{array}$ & 0.12 & 0.08 & 0.18 & & & \\
\hline $\begin{array}{l}\text { Working and claiming (> 50\% of } \\
\text { FTE) }\end{array}$ & 0.12 & 0.11 & 0.13 & & & \\
\hline Male & 0.62 & 1 & 0 & 0.63 & 1 & 0 \\
\hline Age & 58.5 & 58.53 & 58.42 & 58.49 & 58.50 & 58.45 \\
\hline Married & 0.72 & 0.78 & 0.62 & 0.72 & 0.78 & 0.61 \\
\hline Flanders & 0.57 & 0.59 & 0.53 & 0.55 & 0.57 & 0.51 \\
\hline Wallonia & 0.34 & 0.33 & 0.36 & 0.35 & 0.33 & 0.37 \\
\hline Brussels & 0.09 & 0.08 & 0.11 & 0.10 & 0.09 & 0.12 \\
\hline Partner employed & 0.25 & 0.25 & 0.24 & 0.25 & 0.25 & 0.24 \\
\hline Partner claiming & 0.59 & 0.64 & 0.50 & 0.58 & 0.64 & 0.49 \\
\hline Partner working and claiming & 0.07 & 0.07 & 0.07 & 0.06 & 0.06 & 0.07 \\
\hline White collar & 0.61 & 0.56 & 0.69 & 0.68 & 0.67 & 0.70 \\
\hline Primary sector of activity & 0.3 & 0.04 & 0.02 & 0.03 & 0.04 & 0.02 \\
\hline Secondary sector of activity & 0.27 & 0.37 & 0.10 & 0.25 & 0.35 & 0.09 \\
\hline Tertiary sector of activity & 0.69 & 0.59 & 0.87 & 0.71 & 0.61 & 0.89 \\
\hline Mean of assimilated days & 24.32 & 22.45 & 27.43 & 18.02 & 16.02 & 21.40 \\
\hline Lifetime earnings/100,000 & 66.24 & 77.36 & 47.83 & 69.74 & 83.56 & 46.36 \\
\hline Eligible EEA & 0.19 & 0.23 & 0.14 & 0.19 & 0.22 & 0.13 \\
\hline Eligible SEA & 0.01 & 0 & 0.02 & 0.01 & 0 & 0.02 \\
\hline Eligible pension bonus & 0.12 & 0.12 & 0.10 & 0.11 & 0.11 & 0.10 \\
\hline Number of observations & 88,217 & 55,002 & 33,215 & 60,127 & 38,007 & 22,120 \\
\hline Number of individuals & 27,351 & 17,247 & 10,104 & 19,816 & 12,676 & 7,140 \\
\hline
\end{tabular}




\subsubsection{ECONOMETRIC MODEL}

We use a probit model in order to study the determinants of the labor force exit and the benefit claiming decisions ${ }^{212}$.

$$
\begin{aligned}
Y_{i t}^{*}=\alpha+\beta W_{i}^{\prime} & +\beta W_{i t}^{\prime}+\beta X_{i t-1}^{\prime}+\beta Z_{i t}^{\prime}+\sum_{j=56}^{64} \beta \text { age } j_{i t} \\
& +\sum_{j=2005}^{2010} \beta \text { year }_{i t}+\varepsilon_{i t}
\end{aligned}
$$

Where $Y_{i t}^{*}$ refers to the latent probability of labor force exit or benefit claiming for individual $\mathrm{i}$ in period t. $W_{i}^{\prime}$ is a vector of time-constant personal characteristics, $W_{i t}^{\prime}$ is a vector of time-varying personal and partner's characteristic, $X_{i t-1}^{\prime}$ is a vector of timevarying job-related characteristics and $Z_{i t}^{\prime}$ is a vector of time-varying social security eligibility variables. The observation period is $2005-2010$ and each individual is observed for a maximum of 7 periods. ${ }^{213} \varepsilon_{i t}$ is the idiosyncratic error term. We cluster the standard errors at the individual level in order to correct for heteroskedasticity.

\footnotetext{
212 Although the results of the Breusch-Pagan-Lagrange multiplier test indicate that panel data methods should be used, we choose not to use panel methods such as the standard fixed and random effects models because we find little variation of our variables within individuals (which may cause selection bias) and because we expect the individual heterogeneity to be correlated with our explanatory variables. We perform extension and validity checks by using a correlated random effect model (CRE). The results from this model essentially confirm our findings with the probit model. Since the results from our probit and CRE probit are very similar, we present the results of the CRE model in the appendix.

213 The average number of observations per individual is 4.45 for individuals in the sample for the labor force exit decision analysis and 4.25 for individuals in the benefit claiming decision analysis (see section 5.1.3).
} 


\subsection{RESULTS}

\subsubsection{DETERMINANTS OF LABOR FORCE EXIT}

In this section, we present the results of our analysis on the determinants of the labor force exit decision. Table 5.3 displays the average marginal effects differentiated by sex for our two model specifications. The first specification includes a binary variable indicating whether the individual is claiming social security benefits at the end of period t- 1 and its interaction with the work intensity variable (full-time, part-time and less than or equal to 50 percent of FTE and part-time and more than 50 percent of FTE). The second specification splits up the above-mentioned claiming variable into five different claims: old-age pension, unemployment, disability, time credit and other ${ }^{214}$.

In the first model specification, we find that working part-time (regardless of the claim status) and claiming benefits (regardless of work intensity) significantly increases the probability of labor force exit for both sexes (with a stronger effect for men than for women). We find a negative sign for the interaction of working part-time and claiming, indicating that the combination of the two variables decreases the labor force exit probability of the distinct and separate effects of working part-time and claiming benefits. Overall, we find that men who are claimers and work part-time (regardless of the proportion of FTE) have the highest labor force exit probability among men (see figure 5.8). Moreover, we find that women who are claimers and work full-time have the highest labor force exit probability among women. These results coincide with the empirical

\footnotetext{
${ }^{214}$ Other claims in the group work and claim mostly include conventional early retirement or work injury benefits and a smaller part of these claims include work disease and social assistance. Conventional early retirement claims are absent from this variable because these claims are very rare in the work and claim status. Thus they are very low and are thus included in the other category. In the second model specification, we do not include an interaction term between the types of claim and the work intensity because of multicollinearity concerns.
} 
evidence of section 5.3.2 that showed higher labor force exit hazard rates for workers in the group work and claim compared to workers in the group work and no claim for both fulltimers and part-timers.

Figure 5.8: Predicted labor force exit probability by claiming status, part-time work and sex

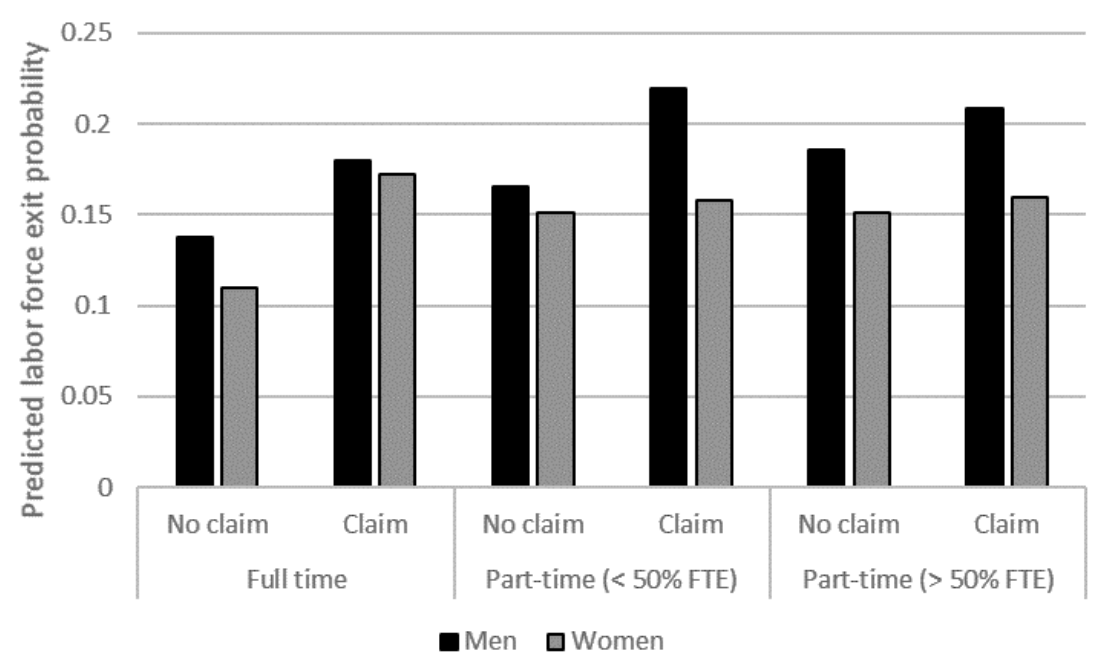

Source: Authors' own calculations using CBSS dataset

We take the analysis one step further in the second specification as we split up the claiming variable into five different types of claims. We find that the effect of benefit claiming on labor force exit probability varies according to the type of claim. Figure 5.9 displays different predicted labor force exit probability by claiming status and sex. For men, it is mostly the time credit and the unemployment claims that lead to the highest predicted probability of labor force exit. For women, it is mostly the unemployment and the disability claims that have the highest predicted labor force exit probability. Our results thus coincide with the results of Albanese et. al. (2015) who find that time credit claimers who are eligible for old-age pension have a higher labor force exit probability compared to other workers. 
Figure 5.9: Predicted labor force exit probabilities of workers in the group work and claim by types of claim and sex

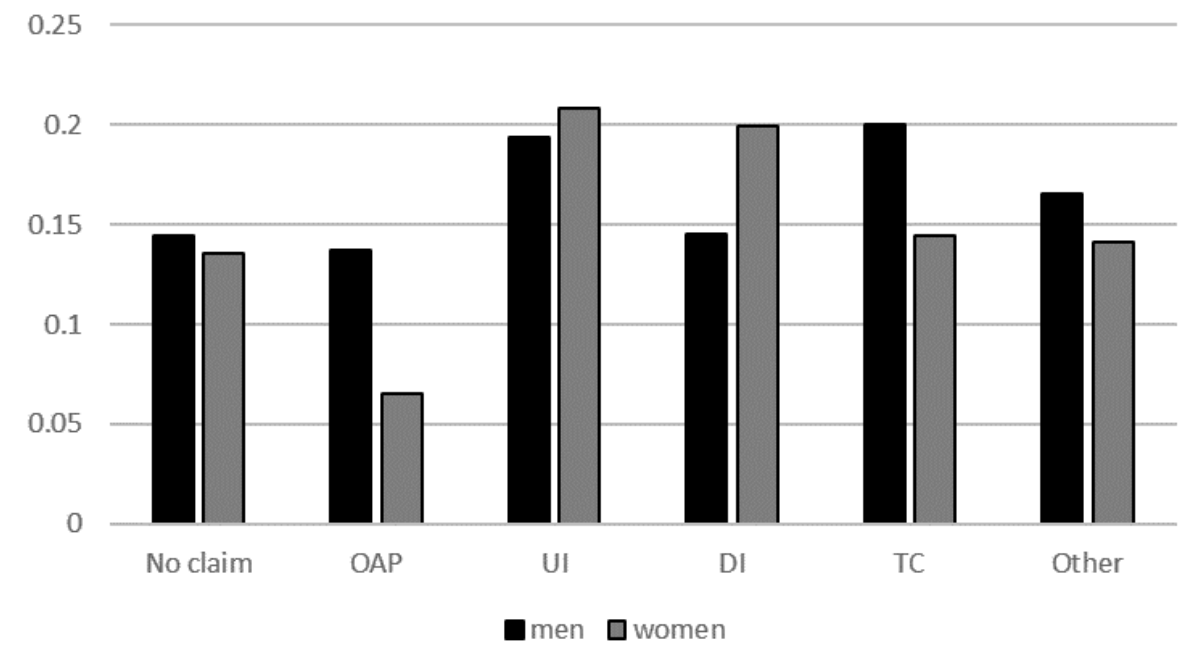

Source: Authors' own calculations using CBSS dataset.

Note: TC stands for time credit, DI stands for disability insurance, UI stands for unemployment insurance, OAP stands for old-age pension.

We find that being a male corresponds to a lower probability of labor force exit. Living in Wallonia or Brussels significantly decreases the labor force exit probability compared to living in Flanders ${ }^{215}$. Being married does not have a significant impact on labor force exit. Nevertheless, having a partner who is employed significantly decreases the labor force exit probability of men. Having a partner who is claiming benefits significantly increases the probability of labor force exit for women but not for men. Having a partner who is working and claiming benefits increases the probability of labor force exit for men but not for women.

For men, being a white-collar worker decreases the probability of labor force exit as opposed to being a blue-collar worker. Working in the primary (for men only) or tertiary

\footnotetext{
${ }^{215}$ This arises from the fact that the intensity of part-time work is not included in the model. Indeed, while the proportion of part-time work is only slightly higher outside of Flanders than inside, the intensity of work inside the group of part-timers differs largely, with part-timers in Flanders working for a higher proportion of a full-time equivalent compared to part-timers outside of Flanders. Therefore, the coefficient of Flanders becomes negative because it captures the effect of work intensity in the part-time category.
} 
rather than in the secondary sector of activity decreases the labor force exit probability of workers. Higher lifetime earnings increases the probability of labor force exit, but at a decreasing rate. A higher number of average assimilated days per year increases the labor force exit probability, which might be an indicator of its correlation with health and unobservables such as productivity, demand for leisure, motivations, etc.

As expected, being eligible for (early) retirement significantly increases the probability of labor force exit, which concurs with the findings of Jousten and Tarantchenko (2014) and Benítez-Silva and Heiland (2008). Even after controlling for eligibility at the early and statutory eligibility ages of old-age pension, there are still peaks of the labor force exit probability at ages 58 and 60, the key eligibility ages in the conventional early retirement and in the old-age pension programs. There are two reasons that could explain this phenomenon. First, since we do not have access to the necessary job and career characteristics needed to build a "true" eligibility indicator for conventional early retirement, our age indicators might capture this "true" eligibility effect on the retirement decision of workers. Second, this result might be a representation of the age reference point theory according to which retirement bunching at the statutory eligibility age cannot only be explained by eligibility alone as this age also serves as a retirement reference point for workers (see chapter four and Seibold, 2017). Therefore, some individuals might exit the labor force at these ages, even though they are not eligible to claim early retirement benefits because the timing is endorsed by the government.

Finally, we observe that the labor force exit probability generally increases with age, possibly because of a worsening of the individual's health status, which is itself negatively correlated with age. Moreover, the labor force exit probability decreases with time, possibly indicating that the reforms aimed at reducing early retirement have fulfilled their role of closing early labor force exit pathways. 
Table 5.3: Determinants of labor force exit

\begin{tabular}{|c|c|c|c|c|c|c|}
\hline & \multicolumn{3}{|c|}{$\begin{array}{c}\text { Model specification } \\
(1) \\
\end{array}$} & & \multicolumn{2}{|c|}{$\begin{array}{c}\text { Model specification } \\
(2) \\
\end{array}$} \\
\hline & \multirow{2}{*}{ Main } & \multicolumn{2}{|c|}{ By sex } & \multirow{2}{*}{ Main } & \multicolumn{2}{|c|}{ By sex } \\
\hline & & Male & Female & & Male & Female \\
\hline $\begin{array}{l}\text { Part-time contract }(\leq 50 \% \\
\text { of FTE) }\end{array}$ & $\begin{array}{l}0.035^{* * *} \\
(0.005)\end{array}$ & $\begin{array}{l}0.031^{* * *} \\
(0.011)\end{array}$ & $\begin{array}{l}0.021^{* * *} \\
(0.006)\end{array}$ & $\begin{array}{l}0.041^{* * *} \\
(0.005)\end{array}$ & $\begin{array}{c}0.035^{* * *} \\
(0.008)\end{array}$ & $\begin{array}{l}0.039^{* * *} \\
(0.006)\end{array}$ \\
\hline $\begin{array}{l}\text { Part-time contract (> 50\% } \\
\text { of FTE) }\end{array}$ & $\begin{array}{l}0.039^{* * *} \\
(0.004)\end{array}$ & $\begin{array}{l}0.039 * * * \\
(0.006)\end{array}$ & $\begin{array}{l}0.022^{* * *} \\
(0.006)\end{array}$ & $\begin{array}{l}0.041^{* * *} \\
(0.004)\end{array}$ & $\begin{array}{l}0.023^{* * *} \\
(0.007)\end{array}$ & $\begin{array}{l}0.038^{* * *} \\
(0.005)\end{array}$ \\
\hline Claiming & $\begin{array}{l}0.038^{* * *} \\
(0.003)\end{array}$ & $\begin{array}{l}0.039^{* * *} \\
(0.004)\end{array}$ & $\begin{array}{l}0.023^{* * *} \\
(0.005)\end{array}$ & & & \\
\hline Old-age pension (OAP) & & & & $\begin{array}{c}-0.027^{* * *} \\
(0.009)\end{array}$ & $\begin{array}{l}-0.008 \\
(0.014)\end{array}$ & $\begin{array}{c}-0.093^{* * *} \\
(0.024)\end{array}$ \\
\hline Unemployment (UI) & & & & $\begin{array}{l}0.059^{* * *} \\
(0.005)\end{array}$ & $\begin{array}{l}0.047^{* * *} \\
(0.005)\end{array}$ & $\begin{array}{l}0.065^{* * *} \\
(0.009)\end{array}$ \\
\hline Disability (DI) & & & & $\begin{array}{l}0.033^{* *} \\
(0.014)\end{array}$ & $\begin{array}{c}0.001 \\
(0.018)\end{array}$ & $\begin{array}{l}0.058^{* * *} \\
(0.017)\end{array}$ \\
\hline Time credit (TC) & & & & $\begin{array}{c}0.031^{* * *} \\
(0.005)\end{array}$ & $\begin{array}{c}0.053^{* * *} \\
(0.007)\end{array}$ & $\begin{array}{c}0.009 \\
(0.006)\end{array}$ \\
\hline Other & & & & $\begin{array}{l}0.019^{* * *} \\
(0.006)\end{array}$ & $\begin{array}{l}0.021^{* * *} \\
(0.007)\end{array}$ & $\begin{array}{c}0.006 \\
(0.011)\end{array}$ \\
\hline Male & $\begin{array}{l}-0.007^{* *} \\
(0.003)\end{array}$ & & & $\begin{array}{l}-0.008^{* *} \\
(0.003)\end{array}$ & & \\
\hline Married & $\begin{array}{l}0.006 \\
(0.005)\end{array}$ & $\begin{array}{l}0.005 \\
(0.006)\end{array}$ & $\begin{array}{l}0.006 \\
(0.007)\end{array}$ & $\begin{array}{c}0.006 \\
(0.005)\end{array}$ & $\begin{array}{c}0.005 \\
(0.006)\end{array}$ & $\begin{array}{c}0.006 \\
(0.007)\end{array}$ \\
\hline Wallonia & $\begin{array}{l}-0.016^{* * *} \\
(0.003)\end{array}$ & $\begin{array}{l}-0.020^{* * *} \\
(0.003)\end{array}$ & $\begin{array}{l}-0.011^{* * *} \\
(0.004)\end{array}$ & $\begin{array}{c}-0.016^{* * *} \\
(0.003)\end{array}$ & $\begin{array}{c}-0.020^{* * *} \\
(0.003)\end{array}$ & $\begin{array}{c}-0.012^{* * *} \\
(0.004)\end{array}$ \\
\hline Brussels & $\begin{array}{l}-0.022^{* * *} \\
(0.004)\end{array}$ & $\begin{array}{l}-0.027^{* * *} \\
(0.006)\end{array}$ & $\begin{array}{l}-0.019^{* * *} \\
(0.007)\end{array}$ & $\begin{array}{c}-0.022^{* * *} \\
(0.004)\end{array}$ & $\begin{array}{c}-0.028^{* * *} \\
(0.006)\end{array}$ & $\begin{array}{c}-0.020^{* * *} \\
(0.007)\end{array}$ \\
\hline Partner employed & $\begin{array}{l}-0.021^{* * *} \\
(0.005)\end{array}$ & $\begin{array}{l}-0.026^{* * *} \\
(0.008)\end{array}$ & $\begin{array}{l}-0.011 \\
(0.009)\end{array}$ & $\begin{array}{c}-0.020^{* * *} \\
(0.005)\end{array}$ & $\begin{array}{c}-0.026^{* * *} \\
(0.008)\end{array}$ & $\begin{array}{l}-0.009 \\
(0.009)\end{array}$ \\
\hline Partner claiming & $\begin{array}{l}0.017^{* * *} \\
(0.005)\end{array}$ & $\begin{array}{l}0.009 \\
(0.007)\end{array}$ & $\begin{array}{l}0.033^{* * *} \\
(0.008)\end{array}$ & $\begin{array}{l}0.017^{* * *} \\
(0.005)\end{array}$ & $\begin{array}{c}0.009 \\
(0.007)\end{array}$ & $\begin{array}{l}0.035^{* * *} \\
(0.008)\end{array}$ \\
\hline $\begin{array}{l}\text { Partner working and } \\
\text { claiming }\end{array}$ & $\begin{array}{l}0.011 \\
(0.008)\end{array}$ & $\begin{array}{l}0.026^{* * *} \\
(0.010)\end{array}$ & $\begin{array}{l}-0.014 \\
(0.012)\end{array}$ & $\begin{array}{c}0.011 \\
(0.008)\end{array}$ & $\begin{array}{l}0.025^{* * *} \\
(0.010)\end{array}$ & $\begin{array}{l}-0.016 \\
(0.012)\end{array}$ \\
\hline White collar & $\begin{array}{l}-0.043^{* * *} \\
(0.003)\end{array}$ & $\begin{array}{l}-0.055^{* * *} \\
(0.004)\end{array}$ & $\begin{array}{l}-0.019^{* * *} \\
(0.005)\end{array}$ & $\begin{array}{c}-0.044^{* * *} \\
(0.003)\end{array}$ & $\begin{array}{c}-0.056^{* * *} \\
(0.004)\end{array}$ & $\begin{array}{c}-0.019^{* * *} \\
(0.005)\end{array}$ \\
\hline $\begin{array}{l}\text { Primary sector of } \\
\text { activity }\end{array}$ & $\begin{array}{l}-0.039^{* * *} \\
(0.007)\end{array}$ & $\begin{array}{l}-0.043^{* * *} \\
(0.008)\end{array}$ & $\begin{array}{l}-0.003 \\
(0.012)\end{array}$ & $\begin{array}{c}-0.038^{* * *} \\
(0.007)\end{array}$ & $\begin{array}{c}-0.042^{* * *} \\
(0.008)\end{array}$ & $\begin{array}{l}-0.002 \\
(0.012)\end{array}$ \\
\hline $\begin{array}{l}\text { Tertiary sector of } \\
\text { activity }\end{array}$ & $\begin{array}{l}-0.068^{* * *} \\
(0.003)\end{array}$ & $\begin{array}{l}-0.069^{* * *} \\
(0.003)\end{array}$ & $\begin{array}{l}-0.040^{* * *} \\
(0.006)\end{array}$ & $\begin{array}{c}-0.067^{* * *} \\
(0.003)\end{array}$ & $\begin{array}{c}-0.068^{* * *} \\
(0.003)\end{array}$ & $\begin{array}{c}-0.038^{* * *} \\
(0.006)\end{array}$ \\
\hline Mean of assimilated days & $0.001^{* * *}$ & $0.001^{* * *}$ & $0.000^{* * *}$ & $0.000^{* * *}$ & $0.001^{* * *}$ & $0.000^{* * *}$ \\
\hline
\end{tabular}




\begin{tabular}{|c|c|c|c|c|c|c|}
\hline \multirow{4}{*}{$\begin{array}{l}\text { Lifetime } \\
\text { earnings/100,000 }\end{array}$} & $(0.000)$ & $(0.000)$ & $(0.000)$ & $(0.000)$ & $(0.000)$ & $(0.000)$ \\
\hline & $0.002^{* * *}$ & $0.002^{* * *}$ & $0.002^{* * *}$ & $0.002^{* * *}$ & $0.001^{* * *}$ & $0.002^{* * *}$ \\
\hline & $(0.000)$ & $(0.000)$ & $(0.000)$ & $(0.000)$ & $(0.000)$ & $(0.000)$ \\
\hline & $-0.000^{* * *}$ & $-0.000^{* * *}$ & $-0.000^{* * *}$ & $-0.000^{* * *}$ & $-0.000^{* * *}$ & $-0.000^{* * *}$ \\
\hline & $(0.000)$ & $(0.000)$ & $(0.000)$ & $(0.000)$ & $(0.000)$ & $(0.000)$ \\
\hline \multirow[t]{2}{*}{ Eligible EEA } & $0.066^{* * *}$ & $0.048^{* * *}$ & $0.071^{* * *}$ & $0.068^{* * *}$ & $0.048^{* * *}$ & $0.073^{* * *}$ \\
\hline & $(0.006)$ & $(0.007)$ & $(0.007)$ & $(0.006)$ & $(0.007)$ & $(0.007)$ \\
\hline \multirow[t]{2}{*}{ Eligible SEA } & $0.265^{* * *}$ & & $0.168^{* * *}$ & $0.270^{* * *}$ & & $0.178^{* * *}$ \\
\hline & $(0.023)$ & & $(0.018)$ & $(0.022)$ & & $(0.018)$ \\
\hline \multirow{2}{*}{$\begin{array}{l}\text { Eligible pension } \\
\text { bonus }\end{array}$} & $0.016^{* * *}$ & $0.010^{*}$ & $0.026^{* * *}$ & $0.014^{* * *}$ & 0.009 & $0.022^{* *}$ \\
\hline & $(0.005)$ & $(0.006)$ & $(0.008)$ & $(0.005)$ & $(0.006)$ & $(0.009)$ \\
\hline \multirow{2}{*}{$\begin{array}{l}\text { Number of observations } \\
\text { Pseudo } \mathrm{R}^{2}\end{array}$} & 88,217 & 55,002 & 33,215 & 88,217 & 55,002 & 33,215 \\
\hline & 0.0868 & 0.0874 & 0.0937 & 0.0887 & 0.0896 & 0.0979 \\
\hline
\end{tabular}

Note: Clustered standard errors are in parentheses. The table reports the average marginal effects. ${ }^{* * *}$ $\mathrm{p}<0.01,{ }^{* *} \mathrm{p}<0.05,{ }^{*} \mathrm{p}<0.1$. All regressions contain age and year binary variables, not presented for space reasons. The first specification contains the interaction between the working regime and the claiming variable, not presented in the table.

\subsubsection{DETERMINANTS OF BENEFIT CLAIMING}

In this section, we present our analysis of the determinants of benefit claiming. Table 5.4 displays the average marginal effects differentiated by sex for two dependent variables: one indicating a new claim in the work and claim or no work and claim groups, and the other indicating a new claim in the work and claim group only.

We first look at the determinants of new claims in the work and claim or no work and claim groups. We find that working part-time with a work intensity of less than or equal to 50 percent of a FTE significantly decreases the probability of benefit claim of women, possibly because these women do not meet the eligibility criteria of the majority of early retirement programs (often based on a minimum number of years of career). Being a male slightly decreases the probability of benefit claiming. Living in Wallonia or Brussels decreases the probability of benefit claiming. Being married does not have an impact on benefit claiming but having an employed partner decreases the probability of benefit claiming of men. Having a partner who is claiming benefits increases the claiming 
probability of women. Having a partner who is working and claiming benefits increases the probability of benefit claiming.

Being a white-collar worker, working in the primary or tertiary sectors of activity decreases the probability of benefit claiming compared to being a blue-collar worker or working in the secondary sector of activity, respectively. Having higher lifetime earnings increases the probability of benefit claim at a decreasing pace and a higher number of average assimilated days per year increases the benefit claiming probability. Finally, we find that being eligible for old-age pension at the early or the statutory eligibility age of old-age pension increases the benefit claiming probability of both men and women.

Once again, we find an increasing benefit claiming probability at some specific ages: 58 , 60 and 61 . We do not find a decreasing benefit claiming probability over time, except for the year 2009, which has a significantly higher benefit claiming probability than the year 2005.

Using our second dependent variable, which indicates a new claim from the group work and no claim to the group work and claim, we mostly find similar results except for a few points, which we summarize below. We find that working part-time, regardless of work intensity, decreases the benefit claiming probability and this effect is stronger for women and for part-time work of less than or equal to 50 percent of FTE. Regarding the partner's characteristics, having a partner who works and claims benefits increases the benefit claiming probability of men but not of women. For women, the sector of activity and being eligible at the early eligibility age of old-age pension does not influence the claiming probability. 
Table 5.4: Determinants of benefit claiming

\begin{tabular}{|c|c|c|c|c|c|c|}
\hline & \multicolumn{3}{|c|}{$\begin{array}{l}\text { New claims in group work and } \\
\text { claim or no work and claim }\end{array}$} & \multicolumn{3}{|c|}{ New claims in group work and claim } \\
\hline & \multirow{2}{*}{ Main } & \multicolumn{2}{|c|}{ By sex } & \multirow{2}{*}{ Main } & \multicolumn{2}{|c|}{ By sex } \\
\hline & & Male & Female & & Male & Female \\
\hline $\begin{array}{l}\text { Part-time contract }(\leq 50 \% \\
\text { of FTE) }\end{array}$ & $\begin{array}{c}-0.045^{* * *} \\
(0.007)\end{array}$ & $\begin{array}{l}-0.027 \\
(0.018)\end{array}$ & $\begin{array}{c}-0.025^{* * *} \\
(0.008)\end{array}$ & $\begin{array}{c}-0.067^{* * *} \\
(0.004)\end{array}$ & $\begin{array}{c}-0.038^{* * *} \\
(0.012)\end{array}$ & $\begin{array}{c}-0.061^{* * *} \\
(0.006)\end{array}$ \\
\hline $\begin{array}{l}\text { Part-time contract (> 50\% } \\
\text { of FTE) }\end{array}$ & $\begin{array}{l}-0.004 \\
(0.006)\end{array}$ & $\begin{array}{c}0.004 \\
(0.011)\end{array}$ & $\begin{array}{c}0.004 \\
(0.007)\end{array}$ & $\begin{array}{c}-0.042^{* * *} \\
(0.004)\end{array}$ & $\begin{array}{l}-0.033^{* * *} \\
(0.008)\end{array}$ & $\begin{array}{c}-0.037^{* * *} \\
(0.006)\end{array}$ \\
\hline Male & $\begin{array}{l}-0.009^{*} \\
(0.005)\end{array}$ & & & $\begin{array}{c}0.000 \\
(0.004)\end{array}$ & & \\
\hline Married & $\begin{array}{l}-0.001 \\
(0.007)\end{array}$ & $\begin{array}{c}0.009 \\
(0.008)\end{array}$ & $\begin{array}{l}-0.015 \\
(0.011)\end{array}$ & $\begin{array}{l}-0.002 \\
(0.005)\end{array}$ & $\begin{array}{c}0.007 \\
(0.007)\end{array}$ & $\begin{array}{l}-0.012^{*} \\
(0.007)\end{array}$ \\
\hline Wallonia & $\begin{array}{l}-0.016^{* * *} \\
(0.004)\end{array}$ & $\begin{array}{c}-0.017^{* * *} \\
(0.005)\end{array}$ & $\begin{array}{c}-0.016^{* * *} \\
(0.006)\end{array}$ & $\begin{array}{l}-0.003 \\
(0.003)\end{array}$ & $\begin{array}{l}-0.002 \\
(0.004)\end{array}$ & $\begin{array}{l}-0.006 \\
(0.004)\end{array}$ \\
\hline Brussels & $\begin{array}{c}-0.036 * * * \\
(0.006)\end{array}$ & $\begin{array}{c}-0.044^{* * *} \\
(0.007)\end{array}$ & $\begin{array}{c}-0.036^{* * *} \\
(0.008)\end{array}$ & $\begin{array}{c}-0.019 * * * \\
(0.004)\end{array}$ & $\begin{array}{c}-0.026^{* * *} \\
(0.006)\end{array}$ & $\begin{array}{c}-0.017^{* * *} \\
(0.006)\end{array}$ \\
\hline Partner employed & $\begin{array}{c}-0.023^{* * *} \\
(0.008)\end{array}$ & $\begin{array}{l}-0.019^{*} \\
(0.010)\end{array}$ & $\begin{array}{l}-0.019 \\
(0.013)\end{array}$ & $\begin{array}{l}-0.009 \\
(0.006)\end{array}$ & $\begin{array}{l}-0.001 \\
(0.008)\end{array}$ & $\begin{array}{l}-0.010 \\
(0.009)\end{array}$ \\
\hline Partner claiming & $\begin{array}{c}0.012 \\
(0.007)\end{array}$ & $\begin{array}{c}0.013 \\
(0.009)\end{array}$ & $\begin{array}{l}0.025^{* *} \\
(0.011)\end{array}$ & $\begin{array}{l}-0.004 \\
(0.005)\end{array}$ & $\begin{array}{c}0.003 \\
(0.007)\end{array}$ & $\begin{array}{l}-0.003 \\
(0.008)\end{array}$ \\
\hline $\begin{array}{l}\text { Partner working and } \\
\text { claiming }\end{array}$ & $\begin{array}{c}0.031^{* * *} \\
(0.011)\end{array}$ & $\begin{array}{l}0.035^{* *} \\
(0.014)\end{array}$ & $\begin{array}{c}0.018 \\
(0.017)\end{array}$ & $\begin{array}{c}0.025^{* * *} \\
(0.008)\end{array}$ & $\begin{array}{l}0.023^{* *} \\
(0.011)\end{array}$ & $\begin{array}{c}0.018 \\
(0.012)\end{array}$ \\
\hline White collar & $\begin{array}{c}-0.076^{* * *} \\
(0.004)\end{array}$ & $\begin{array}{c}-0.090^{* * *} \\
(0.006)\end{array}$ & $\begin{array}{c}-0.037^{* * *} \\
(0.007)\end{array}$ & $\begin{array}{c}-0.056^{* * *} \\
(0.003)\end{array}$ & $\begin{array}{c}-0.066^{* * *} \\
(0.005)\end{array}$ & $\begin{array}{c}-0.030^{* * *} \\
(0.005)\end{array}$ \\
\hline $\begin{array}{l}\text { Primary sector of } \\
\text { Activity }\end{array}$ & $\begin{array}{c}-0.041^{* * *} \\
(0.010)\end{array}$ & $\begin{array}{c}-0.047^{* * *} \\
(0.012)\end{array}$ & $\begin{array}{l}-0.004 \\
(0.019)\end{array}$ & $\begin{array}{c}-0.018^{* *} \\
(0.007)\end{array}$ & $\begin{array}{c}-0.020^{* *} \\
(0.009)\end{array}$ & $\begin{array}{l}-0.007 \\
(0.014)\end{array}$ \\
\hline $\begin{array}{l}\text { Tertiary sector of } \\
\text { Activity }\end{array}$ & $\begin{array}{c}-0.061^{* * *} \\
(0.004)\end{array}$ & $\begin{array}{c}-0.067^{* * *} \\
(0.005)\end{array}$ & $\begin{array}{l}-0.021^{* *} \\
(0.010)\end{array}$ & $\begin{array}{c}-0.019^{* * *} \\
(0.003)\end{array}$ & $\begin{array}{c}-0.022^{* * *} \\
(0.004)\end{array}$ & $\begin{array}{l}-0.004 \\
(0.007)\end{array}$ \\
\hline Mean of assimilated days & $\begin{array}{l}0.001^{* * *} \\
(0.000)\end{array}$ & $\begin{array}{c}0.001^{* * *} \\
(0.000)\end{array}$ & $\begin{array}{c}0.000^{* * *} \\
(0.000)\end{array}$ & $\begin{array}{c}0.000^{* * *} \\
(0.000)\end{array}$ & $\begin{array}{l}0.001^{* * *} \\
(0.000)\end{array}$ & $\begin{array}{c}0.000^{* * *} \\
(0.000)\end{array}$ \\
\hline $\begin{array}{l}\text { Lifetime } \\
\text { earnings/100,000 }\end{array}$ & $\begin{array}{c}0.001^{* * *} \\
(0.000)\end{array}$ & $\begin{array}{l}0.001^{* *} \\
(0.000)\end{array}$ & $\begin{array}{c}0.003^{* * *} \\
(0.000)\end{array}$ & $\begin{array}{c}0.000^{* * *} \\
(0.000)\end{array}$ & $\begin{array}{l}-0.000 \\
(0.000)\end{array}$ & $\begin{array}{c}0.001^{* * *} \\
(0.000)\end{array}$ \\
\hline Squared & $\begin{array}{c}-0.000^{* * *} \\
(0.000)\end{array}$ & $\begin{array}{c}-0.000^{* * *} \\
(0.000)\end{array}$ & $\begin{array}{c}-0.000^{* * *} \\
(0.000)\end{array}$ & $\begin{array}{c}-0.000^{* * *} \\
(0.000)\end{array}$ & $\begin{array}{l}-0.000 \\
(0.000)\end{array}$ & $\begin{array}{c}-0.000^{* *} \\
(0.000)\end{array}$ \\
\hline Eligible EEA & $\begin{array}{c}0.076^{* * *} \\
(0.007)\end{array}$ & $\begin{array}{c}0.063^{* * *} \\
(0.010)\end{array}$ & $\begin{array}{c}0.075^{* * *} \\
(0.010)\end{array}$ & $\begin{array}{l}0.012^{* *} \\
(0.006)\end{array}$ & $\begin{array}{l}0.018^{* *} \\
(0.008)\end{array}$ & $\begin{array}{c}0.000 \\
(0.009)\end{array}$ \\
\hline Eligible SEA & $\begin{array}{c}0.318^{* * *} \\
(0.021)\end{array}$ & & $\begin{array}{c}0.293^{* * *} \\
(0.026)\end{array}$ & $\begin{array}{c}0.174^{* * *} \\
(0.018)\end{array}$ & & $\begin{array}{c}0.152^{* * *} \\
(0.021)\end{array}$ \\
\hline $\begin{array}{l}\text { Eligible pension } \\
\text { Bonus }\end{array}$ & $\begin{array}{l}0.016^{* *} \\
(0.007)\end{array}$ & $\begin{array}{l}0.016^{*} \\
(0.009)\end{array}$ & $\begin{array}{c}0.010 \\
(0.013)\end{array}$ & $\begin{array}{c}0.008 \\
(0.006)\end{array}$ & $\begin{array}{c}0.009 \\
(0.008)\end{array}$ & $\begin{array}{c}0.000 \\
(0.011)\end{array}$ \\
\hline Number of observations & 59,998 & 37,936 & 22,062 & 52,560 & 33,124 & 19,436 \\
\hline Pseudo R ${ }^{2}$ & 0.0516 & 0.0593 & 0.0467 & 0.0477 & 0.0587 & 0.0330 \\
\hline
\end{tabular}

Note: Average marginal effects and clustered standard errors are presented. ${ }^{* * *} p<0.01,{ }^{* *} p<0.05,{ }^{*} p<0.1$. 


\subsection{CONCLUSION}

Nowadays, workers face ever more complex incentives for retirement generated by the historical evolution of the social security system and the variety of early labor force exit pathways that co-exist. Additionally, the lack of careful ex-ante and ex-post analysis of the impact of social security reforms and their intertwined effects between programs contributes to worsen the situation. Such a complex social security system sometimes leads workers to - optimally or not - disjoin labor force exit from (early-) retirement benefit claiming. In this chapter, we analyze the dissociation of the retirement decision into a labor force exit and a benefit claiming decision and we explore its impact for research on the retirement decision and for public finance concerns.

First, we decompose the employed and retired groups of individuals into four subgroups based on their benefit claiming status: (i) work and no claim, (ii) work and claim, (iii) no work and claim and (iv) no work and no claim. We present the retirement and claiming trends of Belgian workers aged from 55 to 64 according to our classification using a detailed administrative dataset from the Crossroad Bank of Social Security for years 2004 to 2010. Further, we document the differences between the labor force exit and the benefit claiming hazard rates and analyze the determinants of both decisions separately.

Our results show that employment rates for older men (particularly between ages 55 and 59) and women are overall increasing. These trends are concomitant with a wave of reforms that was aimed at tightening the eligibility criteria of various early retirement programs (mostly unemployment and conventional early retirement) and possibly indicate that these reforms have fulfilled their role of closing early labor force exit routes to increase the effective retirement age. However, once we decompose the employment trends by benefits claiming status, we note that the situation is not as encouraging as it 
seems. Indeed, an important part of the increase in employment rates is attributable to a rise in part-time work (mostly for women) and in the group of workers who are claiming social security benefits (mostly time-credit, disability and unemployment benefits). We also find that the proportion of workers who are claimers has been growing faster than the group of workers who are not claimers between 2004 and 2010. Most importantly, we find that workers who are claimers (resp. individuals in part-time working arrangements) have a higher probability to exit the labor force early compared to workers who are not claimers (resp. workers in full-time working arrangements). Therefore, our results might indicate that allowing for older workers to gradually reduce their working hours (through a program such as the time credit) does not lead to the desired policy effect, that of delaying retirement. Nevertheless, further research is needed on the direction of causality to reach such a conclusion.

In conclusion, when assessing the financial sustainability of the pension system, the decomposition of employment and retirement trends according to the benefit claiming status proves to be of prime relevance. First, social security reforms have the potential to influence both decisions separately because of the high complexity of the Belgian social security system and the intertwined effects between programs. Second, this richer matrix classification in terms of both retirement and claiming status provides a basis for a better understanding of recent labor market trends. Indeed, in light of the importance of the group of workers who claim social security benefits and their tendency to exit the labor force earlier, it is crucial that the mechanisms underlying their retirement behavior be studied carefully. Moreover, labor market decisions are influenced by the option to combine work and benefit claiming, arguably encouraging some workers to stay on the labor force longer, and such an option should be included in the choice set of individuals when analyzing their retirement behavior. Finally, from a public finance point of view, workers who benefit from social security benefits are likely to contribute less to financing 
social security than other workers. Indeed, on top of working fewer hours on average and receiving social security benefits, these workers represent an additional financial burden as the benefits they receive will be credited at the same rate as their last wage in the oldage pension calculation - hence creating differed fiscal liabilities in addition to the current ones due to benefit payouts. Situations where workers are claiming some form of (early) retirement benefit and where old-age pension beneficiaries are continuing to work after age 65 are becoming increasingly common - rendering the borders between work and retirement ever blurrier and calling for a thorough review of the definition of work and retirement. 


\section{REFERENCES}

Albanese, A., Cockx, B., and Thuy, Y. (2020). Working time reductions at the end of the career: Do they prolong the time spent in employment?, Journal of Empirical Economics, 59, 99-141.

Aliaj, A., Flawinne X. , Jousten A., Perelman S. and Shi L. (2016), “Old-Age Employment and Hours of Work Trends: Empirical Analysis for Four European Countries", IZA Discussion Paper 9819

Benítez-Silva, H., and Yin, N. (2009). An empirical study of the effects of social security reforms on benefit claiming behavior and receipt using public-use administrative microdata. Social Security Bulletin, 69(3).

Benitez-Silva, H., and Heiland, F. (2008). Early claiming of social security and labor supply behavior of older Americans. Journal of Applied Economics, 40(23), 2969-2985.

Blöndal, S. et S. Scarpetta (1999), The Retirement Decision in OECD Countries , Documents de travail du Département des Affaires économiques de l'OCDE, $n^{\circ}$ 202, Éditions OCDE, Paris.

Börsch-Supan, A., Bucher-Koenen, T., Kutlu-Koc, V., and Goll, N. (2017, March).

Dangerous Flexibility - Retirement Reforms Reconsidered. MEA Discussion Papers.

Capéau, B., Decoster, A., Maes, S., and Vanheukelom, T. (2018, September 10). Betaalt de taxshift zichzelf terug? Leuvense Economische Standpunten (Short).

Coile, C. (2004). Retirement Incentives and Couple's Retirement Decisions. Topics in Economic Analysis and Policy, 4(1).

Coile, C. (2015). Economic determinants of workers' retirement decisions. Journal of Economic Surveys, 29(4), 830-853. 
Coile, C., Diamond, P., Gruber, J., and Jousten, A. (2002). Delays in claiming social security benefits. Journal of Public Economics, 84, 357-385.

Delfaortrie, S., and Springael, C. $(2018,10$ 5). Instauration de la pension à mi-temps dans le secteur public. Retrieved from Presscenter by Résidence Palace - International Press Center: https://www.presscenter.org/fr/pressrelease/20181005/instauration-de-lapension-a-mi-temps-dans-le-secteur-public

Dellis, A., R. Desmet, A. Jousten, and S. Perelman (2004). Micro- Modeling of Retirement in Belgium. In Social Security Programs and Retirement around the World: MicroEstimation, edited by J. Gruber and D. Wise, 41-98. Chicago: University of Chicago Press.

Diamond, P., and Gruber, J. (1999). Social Security and Retirement in the United States. In D. Wise, Social Security and Retirement Around the World, 437-473. Chicago: University of Chicago Press.

Duval, R. (2003, November). The retirement effects of old-age pension and early retirement schemes in OECD countries. Economics Department Working Papers (370).

European Commission (DG-EMPL). (2018). The 2018 Pension Adequacy Report: Current and future income adequacy in old age in the EU. Country profiles. Luxembourg: Publications Office of the European Union.

Fraikin, A.L., Jousten, A., and Lefèbvre, M. (2020). Social Security Incentives for Retirement in Belgium: An analysis of four decades of change. In Börsch-Supan, A. and Coile, C., Social security around the world. Chicago: University of Chicago Press and NBER.

Gustman, A. L., and Steinmeier, T. L. (2004). Social security, pensions and retirement behavior within the family. Journal of Applied Econometrics, 19(6), 723-737. 
Gustman, A., and Steinmeier, T. (2015). Effects of social security policies on benefit claiming, retirement and saving. Journal of Public Economics, 51-62.

Haben, B. (2010). Financial incentives to postpone retirement and further effects on employment-Evidence from a natural experiment. Journal of Labor Economics, 17, 474486.

Hurd, M. D. (n.d.). The joint retirement decision of husbands and wives. Social Security Bulletin, 59(1), 29-32.

Jousten, A., and Lefbvre, M. (2013). Retirement Incentives in Belgium: Estimations and Simulations Using SHARE Data. De Economist, 253-276.

Jousten, A., and Lefebvre, M. (2016). Older men's labor force participation in Belgium. Netspar Discussion Paper, 06/2016-031.

Jousten, A., and Tarantchenko, E. (2014). New evidence on the social security incentives as drivers of retirement behavior. SSRN Electronic Journal. 10.2139/ssrn.2429287.

La Libre. (2018, September 8). Bacquelaine: "La pension à mi-temps sera possible au plus tard en janvier 2020". Retrieved September 24, 2018, from

http://www.lalibre.be/actu/politique-belge/bacquelaine-la-pension-a-mi-temps-serapossible-au-plus-tard-en-janvier-2020-5b936346cd70ef4bea999aa3

MacInnis, B. (2009). Social security and the joint trends in labor supply and benefits receipt among older men. Center for Retirement Research at Boston College, Working paper 2009-22.

OECD. (2000). Labor Force Statistics 1978-1998. Paris: OECD Publications.

OECD. (2015). 2015 Pension Policy Notes Belgium. Paris: OECD Publishing.

OECD. (2017). Pensions at a Glance 2017: OECD and G20 Indicators. Paris: OECD Publishing. 
Schunk, R. (2013). Within and between Estimates in Random-Effects Models:

Advantages and Drawbacks of Correlated Random Effects and Hybrid Models. The Stata Journal, 13(1), 65-76.

Seibold, A. (2017) Reference Dependence in Retirement Behavior: Evidence from German Pension Discontinuities. mimeo, London School of Economics.

Staubli, S., and Zweimüller, J. (2013). Does raising the early retirement age increase employment of older workers? Journal of Public Economics, 108, 17-32.

Wooldridge, J. (2019, July). Correlated random effects models with unbalanced panels. Journal of Econometrics, 211(1), 137-150. 


\section{APPENDIX}

\section{A.1 CORRELATED RANDOM EFFECT MODEL}

As validity check, we perform the same analysis using a correlated random effect model. The results from this model essentially confirm our findings with the probit model.

The correlated random effect (CRE) model was first introduced by Mundlak (1978) and further developed by Wooldridge $(2010,2019)$. This method unifies the fixed and the random effects approach and relaxes the strict exogeneity assumption of the random effect model. The CRE model thus allows for individual heterogeneity to be correlated with the explanatory variables through the addition of time-averaged versions of the time-varying explanatory variables, which capture such correlations.

$$
\begin{aligned}
& Y_{i t}=\alpha+\beta W_{i}^{\prime}+\beta W_{i t}^{\prime}+\beta X_{i t-1}^{\prime}+\beta Z_{i t}^{\prime}+\sum_{j=56}^{64} \beta a g e j_{i t} \\
& +\sum_{j=2005}^{2010} \beta \text { year } j_{i t}+{\overline{W^{\prime}}}_{i t}+{\overline{X^{\prime}}}_{i t-1}+{\overline{Z^{\prime}}}_{i t}+\mu_{i}+\varepsilon_{i t}
\end{aligned}
$$

Where $Y_{i t}$ refers to the latent probability of labor force exit or benefit claiming for individual $\mathrm{i}$ in period t. $W_{i}^{\prime}$ is a vector of time-constant personal characteristics, $W_{i t}^{\prime}$ is a vector of time-varying personal and partner characteristic, $X_{i t-1}^{\prime}$ is a vector of timevarying job-related characteristics and $Z_{i t}^{\prime}$ is a vector of time-varying social security eligibility variables. The observation period is 2005-2010 and each individual is observed for a maximum of 7 periods. ${ }^{216}$ In the correlated random effects model, we add the time-

\footnotetext{
216 The average number of observations per individual is 4.45 for individuals in the sample for the labor force exit decision analysis and 4.25 for individuals in the benefit claiming decision analysis.
} 
averaged versions of the vectors $W_{i t}^{\prime}, X_{i t}^{\prime}$ and $Z_{i t}^{\prime}$, namely ${\overline{W^{\prime}}}_{i t}, \bar{X}^{\prime}{ }_{i t}$ and ${\overline{Z^{\prime}}}_{i t}$. $\mu_{i}$ is the individual unobserved heterogeneity and $\varepsilon_{i t}$ is the idiosyncratic error term. We assume that both error terms are uncorrelated and normally distributed with mean zero. We cluster the standard errors at the individual level in order to correct for heteroskedasticity.

While we think it is relevant to exploit the panel nature of our dataset, we find little within variation of our covariates and a small number of observation periods per individual. The results of the CRE model must therefore be analyzed with caution.

Table 5.A.1 displays the results of our correlated random effects probit model for the labor force exit dependent variable using our two model specifications and differentiated by sex.

In the correlated random effects probit model, the coefficients indicate the within effect of the covariates on the dependent variable. ${ }^{217}$ We find that transferring to a part-time contract of more than 50 percent of a FTE and starting to claim benefits significantly increases the labor force exit of men but not women (possibly because of a lack of within individual variation for the latter). The results concerning the other covariates are similar to that of the probit model. Using our second specification, we find an additional positive and significant effect of transferring to a part-time contract of less than or equal to 50 percent of FTE for men on the labor force exit probability ${ }^{218}$. Interestingly, we find a negative effect of starting to claim time credit benefits on the probability of labor force exit for women, which might work in favor of the results of Albanese et. al. (2015) who

\footnotetext{
${ }^{217}$ The coefficients of time-varying explanatory variables can be interpreted as the within effect and the coefficients of the time average variables can be interpreted as the difference between the within and the between effects (Schunk, 2013).

218 This is possibly caused by correlation between the detailed claiming variable and our part-time indicator, which captures this correlation in the first specification model.
} 
find that women who claim time credit benefits have a higher probability to stay on the labor force in the first four years of claim. Additionally, we find that claiming unemployment benefits (for men), disability benefits (for women) and old-age pension benefits significantly increases the labor force exit probability.

Table 5.A.1: Determinants of labor force exit - correlated random effects probit model

\begin{tabular}{|c|c|c|c|c|c|c|}
\hline & \multicolumn{3}{|c|}{ Model specification (1) } & \multicolumn{3}{|c|}{ Model specification (2) } \\
\hline & \multirow{2}{*}{ Main } & \multicolumn{2}{|c|}{ By sex } & \multirow{2}{*}{ Main } & \multicolumn{2}{|c|}{ By sex } \\
\hline & & Male & Female & & Male & Female \\
\hline $\begin{array}{l}\text { Part-time contract }(\leq 50 \% \\
\text { of FTE) }\end{array}$ & $\begin{array}{l}0.030^{*} \\
(0.016)\end{array}$ & $\begin{array}{l}0.045 \\
(0.032)\end{array}$ & $\begin{array}{l}-0.009 \\
(0.020)\end{array}$ & $\begin{array}{l}0.053^{* * *} \\
(0.012)\end{array}$ & $\begin{array}{l}0.076^{* * *} \\
(0.016)\end{array}$ & $\begin{array}{l}0.023 \\
(0.018)\end{array}$ \\
\hline $\begin{array}{l}\text { Part-time contract (> 50\% } \\
\text { of FTE) }\end{array}$ & $\begin{array}{l}0.042^{* * *} \\
(0.011)\end{array}$ & $\begin{array}{l}0.084^{* * *} \\
(0.019)\end{array}$ & $\begin{array}{l}-0.006 \\
(0.015)\end{array}$ & $\begin{array}{l}0.029^{* * *} \\
(0.010)\end{array}$ & $\begin{array}{l}0.038^{* * *} \\
(0.013)\end{array}$ & $\begin{array}{l}0.004 \\
(0.015)\end{array}$ \\
\hline Claiming & $\begin{array}{l}0.014^{* *} \\
(0.006)\end{array}$ & $\begin{array}{l}0.021^{* * *} \\
(0.007)\end{array}$ & $\begin{array}{l}-0.007 \\
(0.015)\end{array}$ & & & \\
\hline Time credit (TC) & & & & $\begin{array}{l}-0.018^{*} \\
(0.010)\end{array}$ & $\begin{array}{l}-0.008 \\
(0.014)\end{array}$ & $\begin{array}{l}-0.047^{* * *} \\
(0.017)\end{array}$ \\
\hline Unemployment (UI) & & & & $\begin{array}{l}0.024^{* * *} \\
(0.008)\end{array}$ & $\begin{array}{l}0.029^{* * *} \\
(0.009)\end{array}$ & $\begin{array}{l}0.019 \\
(0.017)\end{array}$ \\
\hline Disability (DI) & & & & $\begin{array}{l}0.046^{*} \\
(0.027)\end{array}$ & $\begin{array}{l}0.000 \\
(0.037)\end{array}$ & $\begin{array}{l}0.085^{* *} \\
(0.037)\end{array}$ \\
\hline Old-age pension (OAP) & & & & $\begin{array}{l}0.108^{* * *} \\
(0.025)\end{array}$ & $\begin{array}{l}0.091^{* * *} \\
(0.033)\end{array}$ & $\begin{array}{l}0.094^{* *} \\
(0.041)\end{array}$ \\
\hline Other & & & & $\begin{array}{l}-0.002 \\
(0.007)\end{array}$ & $\begin{array}{l}0.005 \\
(0.009)\end{array}$ & $\begin{array}{l}-0.019 \\
(0.013)\end{array}$ \\
\hline Male & $\begin{array}{l}-0.013^{* * *} \\
(0.003)\end{array}$ & & & $\begin{array}{l}-0.015^{* * *} \\
(0.003)\end{array}$ & & \\
\hline Married & $\begin{array}{l}0.003 \\
(0.004)\end{array}$ & $\begin{array}{l}0.007 \\
(0.006)\end{array}$ & $\begin{array}{l}-0.002 \\
(0.007)\end{array}$ & $\begin{array}{l}0.004 \\
(0.004)\end{array}$ & $\begin{array}{l}0.006 \\
(0.006)\end{array}$ & $\begin{array}{l}-0.002 \\
(0.007)\end{array}$ \\
\hline Wallonia & $\begin{array}{l}-0.016^{* * *} \\
(0.002)\end{array}$ & $\begin{array}{l}-0.018^{* * *} \\
(0.003)\end{array}$ & $\begin{array}{l}-0.014^{* * *} \\
(0.004)\end{array}$ & $\begin{array}{l}-0.016^{* * *} \\
(0.002)\end{array}$ & $\begin{array}{l}-0.017^{* * *} \\
(0.003)\end{array}$ & $\begin{array}{l}-0.014^{* * *} \\
(0.004)\end{array}$ \\
\hline Brussels & $\begin{array}{l}-0.018^{* * *} \\
(0.004)\end{array}$ & $\begin{array}{l}-0.019 * * * \\
(0.006)\end{array}$ & $\begin{array}{l}-0.016^{* * *} \\
(0.006)\end{array}$ & $\begin{array}{l}-0.019^{* * *} \\
(0.004)\end{array}$ & $\begin{array}{l}-0.018^{* * *} \\
(0.006)\end{array}$ & $\begin{array}{l}-0.017^{* * *} \\
(0.006)\end{array}$ \\
\hline Partner employed & $\begin{array}{l}0.009 \\
(0.012)\end{array}$ & $\begin{array}{l}0.002 \\
(0.016)\end{array}$ & $\begin{array}{l}0.017 \\
(0.018)\end{array}$ & $\begin{array}{l}0.010 \\
(0.012)\end{array}$ & $\begin{array}{l}0.003 \\
(0.016)\end{array}$ & $\begin{array}{l}0.017 \\
(0.018)\end{array}$ \\
\hline Partner claiming & $\begin{array}{l}0.015 \\
(0.013)\end{array}$ & $\begin{array}{l}-0.019 \\
(0.017)\end{array}$ & $\begin{array}{l}0.061^{* * *} \\
(0.019)\end{array}$ & $\begin{array}{l}0.016 \\
(0.013)\end{array}$ & $\begin{array}{l}-0.019 \\
(0.017)\end{array}$ & $\begin{array}{l}0.062^{* * *} \\
(0.019)\end{array}$ \\
\hline $\begin{array}{l}\text { Partner working and } \\
\text { claiming }\end{array}$ & $\begin{array}{l}0.013^{*} \\
(0.008)\end{array}$ & $\begin{array}{l}0.036^{* * *} \\
(0.010)\end{array}$ & $\begin{array}{l}-0.021^{*} \\
(0.012)\end{array}$ & $\begin{array}{l}0.013^{*} \\
(0.008)\end{array}$ & $\begin{array}{l}0.036^{* * *} \\
(0.010)\end{array}$ & $\begin{array}{l}-0.022^{*} \\
(0.012)\end{array}$ \\
\hline White collar & -0.001 & -0.016 & 0.004 & 0.003 & -0.007 & 0.005 \\
\hline
\end{tabular}




\begin{tabular}{llll|lll} 
& $(0.025)$ & $(0.036)$ & $(0.033)$ & $(0.024)$ & $(0.036)$ & $(0.033)$ \\
Primary sector of & 0.012 & -0.009 & 0.041 & 0.012 & -0.010 & 0.046 \\
activity & $(0.035)$ & $(0.041)$ & $(0.063)$ & $(0.035)$ & $(0.041)$ & $(0.063)$ \\
Tertiary sector of & $0.060^{*}$ & 0.035 & $0.107^{*}$ & $0.059^{*}$ & 0.034 & $0.110^{*}$ \\
activity & $(0.033)$ & $(0.040)$ & $(0.060)$ & $(0.033)$ & $(0.039)$ & $(0.060)$ \\
Mean of assimilated days & $0.010^{* * *}$ & $0.013^{* * *}$ & $0.008^{* * *}$ & $0.010^{* * *}$ & $0.013^{* * *}$ & $0.008^{* * *}$ \\
& $(0.000)$ & $(0.000)$ & $(0.000)$ & $(0.000)$ & $(0.000)$ & $(0.000)$ \\
Lifetime & $0.041^{* * *}$ & $0.041^{* * *}$ & $0.057^{* * *}$ & $0.042^{* * *}$ & $0.041^{* * *}$ & $0.058^{* * *}$ \\
earnings/100,000 & $(0.001)$ & $(0.001)$ & $(0.003)$ & $(0.001)$ & $(0.001)$ & $(0.003)$ \\
Squared & $-0.000^{* * *}$ & $-0.000^{* * *}$ & $-0.000^{* * *}$ & $-0.000^{* * *}$ & $-0.000^{* * *}$ & $-0.000^{* * *}$ \\
& $(0.000)$ & $(0.000)$ & $(0.000)$ & $(0.000)$ & $(0.000)$ & $(0.000)$ \\
Eligible EEA & $0.211^{* * *}$ & $0.215^{* * *}$ & $0.211^{* * *}$ & $0.212^{* * *}$ & $0.214^{* * *}$ & $0.211^{* * *}$ \\
& $(0.006)$ & $(0.009)$ & $(0.009)$ & $(0.006)$ & $(0.009)$ & $(0.009)$ \\
Eligible SEA & $0.206^{* * *}$ & & $0.099^{* * *}$ & $0.206^{* * *}$ & & $0.094^{* * *}$ \\
Eligible pension & $(0.020)$ & & $(0.024)$ & $(0.020)$ & & $(0.025)$ \\
bonus & $0.292^{* * *}$ & $0.293^{* * *}$ & $0.276^{* * *}$ & $0.291^{* * *}$ & $0.293^{* * *}$ & $0.273^{* * *}$ \\
& $(0.008)$ & $(0.010)$ & $(0.014)$ & $(0.008)$ & $(0.010)$ & $(0.014)$ \\
\hline Number of observations & 88,217 & 55,002 & 33,215 & 88,217 & 55,002 & 33,215 \\
\hline
\end{tabular}

Note: Clustered standard errors are in parentheses. The table reports the average marginal effects. ${ }^{* * *}$ $\mathrm{p}<0.01,{ }^{* *} \mathrm{p}<0.05,{ }^{*} \mathrm{p}<0.1$.

Table 5.A.2 displays the results of our correlated random effects model for the benefit claiming decision. Unlike our probit model, working as a part-timer does not have an important impact on the probability of claiming benefits. This result reinforces our intuition that the negative correlation we found between part-time work and the probability of claiming benefits is caused by a lower probability of fulfilling the eligibility criteria of early retirement programs. We also find that being eligible at the early eligibility age and statutory eligibility age of old-age pension has a stronger and significant impact on the benefit claiming probability. This is also the case for the pension bonus, except for women in the case of a transfer to the work and claim group. 
Table 5.A.2: Determinants of benefit claiming -correlated random effects probit model

\begin{tabular}{|c|c|c|c|c|c|c|}
\hline & \multicolumn{3}{|c|}{$\begin{array}{l}\text { Dependent variable: new claims } \\
\text { in group work and claim or no work } \\
\text { and claim }\end{array}$} & \multicolumn{3}{|c|}{$\begin{array}{l}\text { Dependent variable: new claims in } \\
\text { group work and claim }\end{array}$} \\
\hline & \multirow{2}{*}{ Main } & \multicolumn{2}{|c|}{ By sex } & \multirow{2}{*}{ Main } & \multicolumn{2}{|l|}{ By sex } \\
\hline & & Male & Female & & Male & Female \\
\hline \multirow{2}{*}{$\begin{array}{l}\text { Part-time contract }(\leq 50 \% \\
\text { of FTE) }\end{array}$} & -0.143 & -0.269 & -0.017 & $-0.233^{*}$ & -0.365 & -0.090 \\
\hline & $(0.180)$ & $(0.315)$ & $(0.240)$ & $(0.132)$ & $(0.230)$ & $(0.178)$ \\
\hline \multirow{2}{*}{$\begin{array}{l}\text { Part-time contract (> 50\% } \\
\text { of FTE) }\end{array}$} & -0.083 & -0.033 & -0.063 & $-0.295^{* * *}$ & $-0.355^{* * *}$ & -0.216 \\
\hline & $(0.131)$ & $(0.184)$ & $(0.195)$ & $(0.095)$ & $(0.134)$ & $(0.143)$ \\
\hline Male & $\begin{array}{l}-0.060^{*} \\
(0.033)\end{array}$ & & & $\begin{array}{l}0.022 \\
(0.024)\end{array}$ & & \\
\hline Married & $\begin{array}{l}-0.055 \\
(0.046)\end{array}$ & $\begin{array}{l}0.017 \\
(0.057)\end{array}$ & $\begin{array}{l}-0.150^{*} \\
(0.077)\end{array}$ & $\begin{array}{l}-0.026 \\
(0.033)\end{array}$ & $\begin{array}{l}0.026 \\
(0.040)\end{array}$ & $\begin{array}{l}-0.097 \\
(0.060)\end{array}$ \\
\hline Wallonia & $\begin{array}{l}-0.103^{* * *} \\
(0.025)\end{array}$ & $\begin{array}{l}-0.089^{* * *} \\
(0.031)\end{array}$ & $\begin{array}{l}-0.150^{* * *} \\
(0.042)\end{array}$ & $\begin{array}{l}-0.012 \\
(0.018)\end{array}$ & $\begin{array}{l}0.001 \\
(0.022)\end{array}$ & $\begin{array}{l}-0.041 \\
(0.034)\end{array}$ \\
\hline Brussels & $\begin{array}{l}-0.196^{* * *} \\
(0.041)\end{array}$ & $\begin{array}{l}-0.207^{* * *} \\
(0.054)\end{array}$ & $\begin{array}{l}-0.228^{* * *} \\
(0.065)\end{array}$ & $\begin{array}{l}-0.097^{* * *} \\
(0.030)\end{array}$ & $\begin{array}{l}-0.112^{* * *} \\
(0.039)\end{array}$ & $\begin{array}{l}-0.098^{*} \\
(0.051)\end{array}$ \\
\hline Partner employed & $\begin{array}{l}0.079 \\
(0.128)\end{array}$ & $\begin{array}{l}0.122 \\
(0.164)\end{array}$ & $\begin{array}{l}0.050 \\
(0.209)\end{array}$ & $\begin{array}{l}0.056 \\
(0.092)\end{array}$ & $\begin{array}{l}0.150 \\
(0.114)\end{array}$ & $\begin{array}{l}-0.053 \\
(0.162)\end{array}$ \\
\hline Partner claiming & $\begin{array}{l}0.056 \\
(0.136)\end{array}$ & $\begin{array}{l}-0.105 \\
(0.173)\end{array}$ & $\begin{array}{l}0.331 \\
(0.224)\end{array}$ & $\begin{array}{l}-0.006 \\
(0.097)\end{array}$ & $\begin{array}{l}-0.001 \\
(0.119)\end{array}$ & $\begin{array}{l}0.034 \\
(0.172)\end{array}$ \\
\hline $\begin{array}{l}\text { Partner working and } \\
\text { claiming }\end{array}$ & $\begin{array}{l}0.173^{* *} \\
(0.081)\end{array}$ & $\begin{array}{l}0.279^{* * *} \\
(0.102)\end{array}$ & $\begin{array}{l}-0.036 \\
(0.135)\end{array}$ & $\begin{array}{l}0.123^{* *} \\
(0.057)\end{array}$ & $\begin{array}{l}0.119^{*} \\
(0.070)\end{array}$ & $\begin{array}{l}0.077 \\
(0.104)\end{array}$ \\
\hline White collar & $\begin{array}{l}-0.060 \\
(0.272)\end{array}$ & $\begin{array}{l}-0.271 \\
(0.391)\end{array}$ & $\begin{array}{l}-0.261 \\
(0.395)\end{array}$ & $\begin{array}{l}-0.260 \\
(0.190)\end{array}$ & $\begin{array}{l}-0.362 \\
(0.268)\end{array}$ & $\begin{array}{l}-0.370 \\
(0.288)\end{array}$ \\
\hline $\begin{array}{l}\text { Primary sector of } \\
\text { activity }\end{array}$ & $\begin{array}{l}0.436 \\
(0.448)\end{array}$ & $\begin{array}{l}0.865 \\
(0.567)\end{array}$ & $\begin{array}{l}-0.435 \\
(0.761)\end{array}$ & $\begin{array}{l}0.206 \\
(0.333)\end{array}$ & $\begin{array}{l}0.317 \\
(0.418)\end{array}$ & $\begin{array}{l}-0.009 \\
(0.571)\end{array}$ \\
\hline $\begin{array}{l}\text { Tertiary sector of } \\
\text { activity }\end{array}$ & $\begin{array}{l}0.749^{*} \\
(0.431)\end{array}$ & $\begin{array}{l}1.313^{* *} \\
(0.549)\end{array}$ & $\begin{array}{l}-0.511 \\
(0.719)\end{array}$ & $\begin{array}{l}0.478 \\
(0.320)\end{array}$ & $\begin{array}{l}0.657 \\
(0.406)\end{array}$ & $\begin{array}{l}0.031 \\
(0.529)\end{array}$ \\
\hline Mean of assimilated days & $\begin{array}{l}-0.130^{* * *} \\
(0.010)\end{array}$ & $\begin{array}{l}-0.129^{* * *} \\
(0.016)\end{array}$ & $\begin{array}{l}-0.105^{* * *} \\
(0.013)\end{array}$ & $\begin{array}{l}-0.083^{* * *} \\
(0.007)\end{array}$ & $\begin{array}{l}-0.087^{* * *} \\
(0.010)\end{array}$ & $\begin{array}{l}-0.066^{* * *} \\
(0.009)\end{array}$ \\
\hline $\begin{array}{l}\text { Lifetime } \\
\text { earnings/100,000 }\end{array}$ & $\begin{array}{l}0.532^{* * *} \\
(0.013)\end{array}$ & $\begin{array}{l}0.480^{* * *} \\
(0.014)\end{array}$ & $\begin{array}{l}0.878^{* * *} \\
(0.033)\end{array}$ & $\begin{array}{l}0.334^{* * *} \\
(0.009)\end{array}$ & $\begin{array}{l}0.309^{* * *} \\
(0.009)\end{array}$ & $\begin{array}{l}0.595^{* * *} \\
(0.027)\end{array}$ \\
\hline Squared & $-0.001^{* * *}$ & $-0.001^{* * *}$ & $-0.003^{* * *}$ & $-0.001^{* * *}$ & $-0.000^{* * *}$ & $-0.002^{* * *}$ \\
\hline
\end{tabular}




\begin{tabular}{llll|lll} 
& $(0.000)$ & $(0.000)$ & $(0.000)$ & $(0.000)$ & $(0.000)$ & $(0.000)$ \\
Eligible EEA & $1.482^{* * *}$ & $1.515^{* * *}$ & $1.708^{* * *}$ & $0.303^{* * *}$ & $0.375^{* * *}$ & $0.436^{* * *}$ \\
& $(0.073)$ & $(0.094)$ & $(0.121)$ & $(0.057)$ & $(0.071)$ & $(0.101)$ \\
Eligible SEA & $2.567^{* * *}$ & & $1.887^{* * *}$ & $1.606^{* * *}$ & & $1.674^{* * *}$ \\
& $(0.227)$ & & $(0.296)$ & $(0.178)$ & & $(0.241)$ \\
Eligible pension & $1.941^{* * *}$ & $1.847^{* * *}$ & $1.951^{* * *}$ & $0.292^{* * *}$ & $0.303^{* * *}$ & 0.022 \\
bonus & $(0.083)$ & $(0.097)$ & $(0.169)$ & $(0.057)$ & $(0.064)$ & $(0.131)$ \\
& & & & & & \\
\hline Number of observations & 59,998 & 37,936 & 22,062 & 52,560 & 33,124 & 19,436 \\
\hline
\end{tabular}

Note: Clustered standard errors are in parentheses. The table reports the average marginal effects. ${ }^{* * *}$ $\mathrm{p}<0.01,{ }^{* *} \mathrm{p}<0.05,{ }^{*} \mathrm{p}<0.1$. 


\section{THE OLD-AGE PENSION HOUSEHOLD REPLACEMENT RATE FOR PENSIONERS IN ONE- EARNER HOUSEHOLDS}

Alessio Brown and Anne-Lore Fraikin 


\subsection{INTRODUCTION}

Public old-age pension systems have four central objectives. From an individual's viewpoint, they should provide (I) a consumption smoothing mechanism over the life cycle and (II) an insurance against the uncertainty of life expectancy after retirement. From a public policy perspective, they should serve as (III) a poverty alleviation mechanism for old age and (IV) an income redistribution tool among the elderly (Barr and Diamond, 2006). However, faced with population ageing, the prime focus of many European governments in recent decades has been to reform their pension systems to ensure financial sustainability (Grech, 2013). Next to these pressing sustainability concerns, pension adequacy ${ }^{219}$ matters have traditionally been left aside in both public policy and research.

In Belgium, the government has implemented numerous social security reforms over the last decade curbing incentives for early retirement with the aim of ensuring the sustainability of the country's pension system. At the same time, the poverty rate of the country's elderly population has been decreasing thanks to higher female activity rates and numerous discretional increases in minimum and social pensions. However, there remains a substantial gender poverty gap and the average old-age pension benefits of married women is still lower than that of single women (Hindriks, 2015).

In this chapter, we take a closer look at the retirement incentives of workers in one-earner households, i.e. households composed of one earner and one partner who is financially dependent. We leave aside the study of the work (dis)incentives that are potentially associated with these benefits for the financial dependent spouse in the household. While

\footnotetext{
${ }^{219}$ An adequate pension system provides a retirement income that prevents from old-age poverty and allows for consumption smoothing at older ages (Holzmann and Hinz, 2005).
} 
relevant, such analysis should be considered separately as the incentives faced by these individuals are very different from these faced by other types of workers. Married ${ }^{220}$ workers in one-earner households are automatically granted a more generous replacement rate in the calculation of their old-age pension benefits, called the household replacement rate. In case of divorce or death, the replacement rate of the old-age pension is brought down to the isolated replacement rate for the main earner and some divorcee entitlements or the survivor pension are granted to the financially dependent spouse. ${ }^{221}$ Following the rise of the two-earner household model and pension sustainability concerns, many European countries abolished these benefits and the Belgian Pension Reform Committee has recommended to remove the household replacement rate, except for minimum pensions (Pension Reform Committee 2020-2040, 2014). Specifically, we test whether the household replacement rate entails a work (dis)incentive mechanism promoting (harming) pension sustainability and furthermore, we analyse the role of the household replacement rate in ensuring pension adequacy from a public policy perspective.

While contributing to the growing literature focusing on both pension adequacy and pension sustainability, this chapter provides valuable insights to guide evidence-based policy making on this policy challenge. In addition, we provide an ex-ante impact evaluation of policy changes - reducing the generosity or eliminating the household replacement rate benefits - on the retirement decisions and poverty measures among the elderly.

Our analysis shows that in contrast to spousal characteristics, financial incentives have a significant effect on the retirement decision across all household types. Consistently with

\footnotetext{
220 The household replacement rate is not granted to legally cohabiting partners.

${ }^{221}$ Evidence from our micro data demonstrates that divorces at older ages in our sample are scarce.
} 
results from chapter four, we find a significant impact of our social security wealth and accrual variables, which work in opposite directions. Nonetheless, the overall incentives created by the household replacement rate do not confirm our expectations that the household replacement rate creates a work (dis)incentive as they are very limited. Overall, despite the positive poverty and distributional aspects of this policy, our analysis supports the reform proposal of removing the household replacement rate.

In the next section of this chapter, we outline the role of the household replacement rate benefits in Belgium and review the relevant literature for our analysis (i) the retirement behavior of an individual in a one-earner household and (ii) the impact of additional benefits based on the dependent status of the partner on retirement incentives. In the third section, we present our empirical strategy including information on the dataset, the construction of financial incentive measures and our sampling methodology. Sections 6.4 and 6.5 present the model, the results of our analysis and discuss them. Section 6.6 concludes.

\subsection{CONTEXTUAL INFORMATION}

\subsubsection{THE HOUSEHOLD REPLACEMENT RATE}

Belgium is one of the last few European countries that still (indirectly) compensates individuals for household and care work by providing additional old-age pension benefits to their spouse. In Belgium, the household replacement rate of 75 percent is granted to pensioners in one-earner households ${ }^{222}$, provided the couple is married and

\footnotetext{
222 One-earner households are households composed of one earner and one partner who is financially dependent. Two-earners households where the lowest earner earns a very low wage, i.e. that is below the threshold for the household replacement rate, are also included in our one-earner household definition.
} 
the earnings of the dependent spouse do not exceed a certain threshold. ${ }^{223}$ In comparison, pensioners who are single or in two-earner households ${ }^{222}$ are entitled to receive the isolated replacement rate of 60 percent.

Many European countries have gotten rid of benefit programs for financially dependent spouses ensuing pension sustainability concerns, which call for a reduction in the overall generosity of the system. At the time of writing, only eight European countries still propose such programs or have recently removed them: Belgium, the United Kingdom (no new claims since 2010), France (no new claims since 2011), Portugal, Norway, the Netherlands (no new claims since 2015), Ireland and Cyprus. ${ }^{224}$ Nevertheless, these types of benefits are much more prominent outside of Europe as different variants exist in the USA, Argentina, Canada, Chile, Hong Kong, Colombia, Guatemala, Mexico, Peru, Japan, Jordan, New-Zealand, the Philippines, Taiwan and South-Korea, among others.

Belgium is the European country with the highest proportion of pensioners receiving additional benefits for financially dependent spouse, the so-called household replacement rate benefits ${ }^{225}$. Indeed, although the proportion of male ${ }^{226}$ beneficiaries of the household replacement rate has been declining continuously in the last decades, it still amounts to

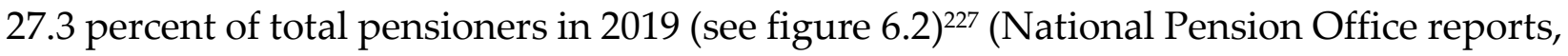
1992-2019). In total, pensions paid at the household replacement rate account for 18.8

\footnotetext{
${ }^{223}$ See chapter two for detailed regulation of the program.

224 See the appendix for a table describing the main features of these programs.

${ }^{225}$ See the appendix for an international comparison of the proportion of pensioners receiving additional benefits based on the financially dependent status of the partner.

${ }^{226}$ In 99.5 percent of cases, it is the man who is the earner in one-earner couples (Berghman, Curvers, Palmans and Peeters, 2007).

227 Concurrently, the number of stay at home moms has been decreasing from approximately 1,222,000 in 1986 to approximately 440,000 in 2016 (Statistics Belgium, 2015).
} 
percent of total old age pension benefits (National Pension Office official statistics reports, 2019).

Figure 6.2: Evolution of pension benefits by replacement rate, marital status and gender (1992-2019)

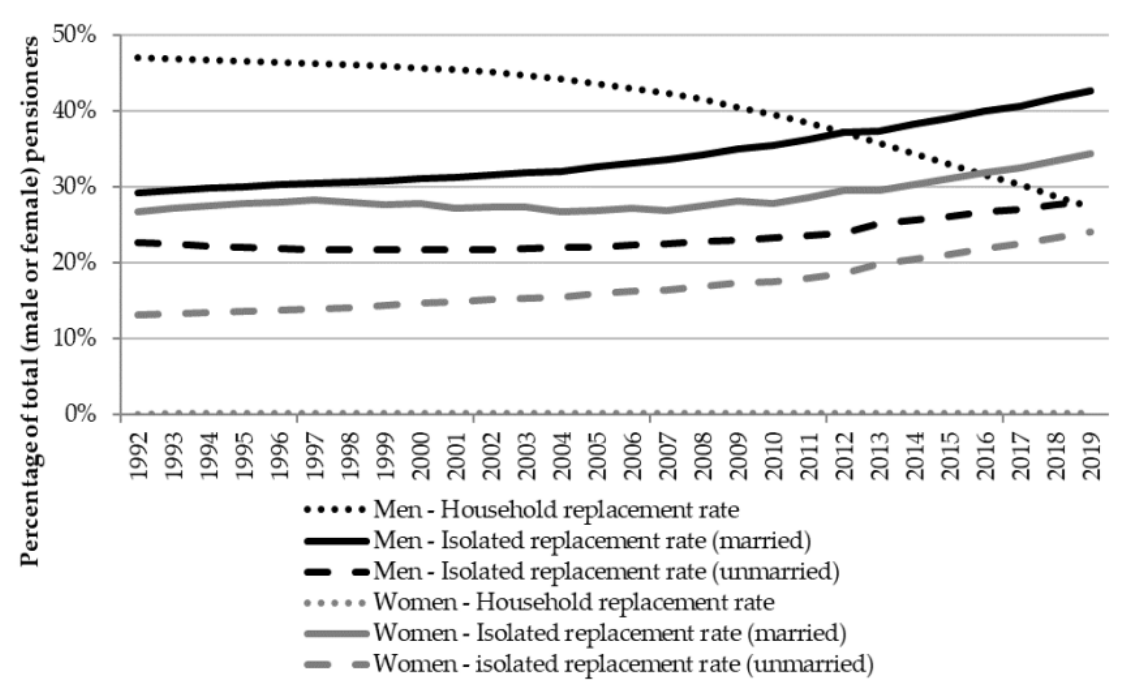

Source: National Pension Office annual reports (1992-2019)

Figure 6.3: Proportion of pension by amount, replacement rate and gender (average 2002-2019)

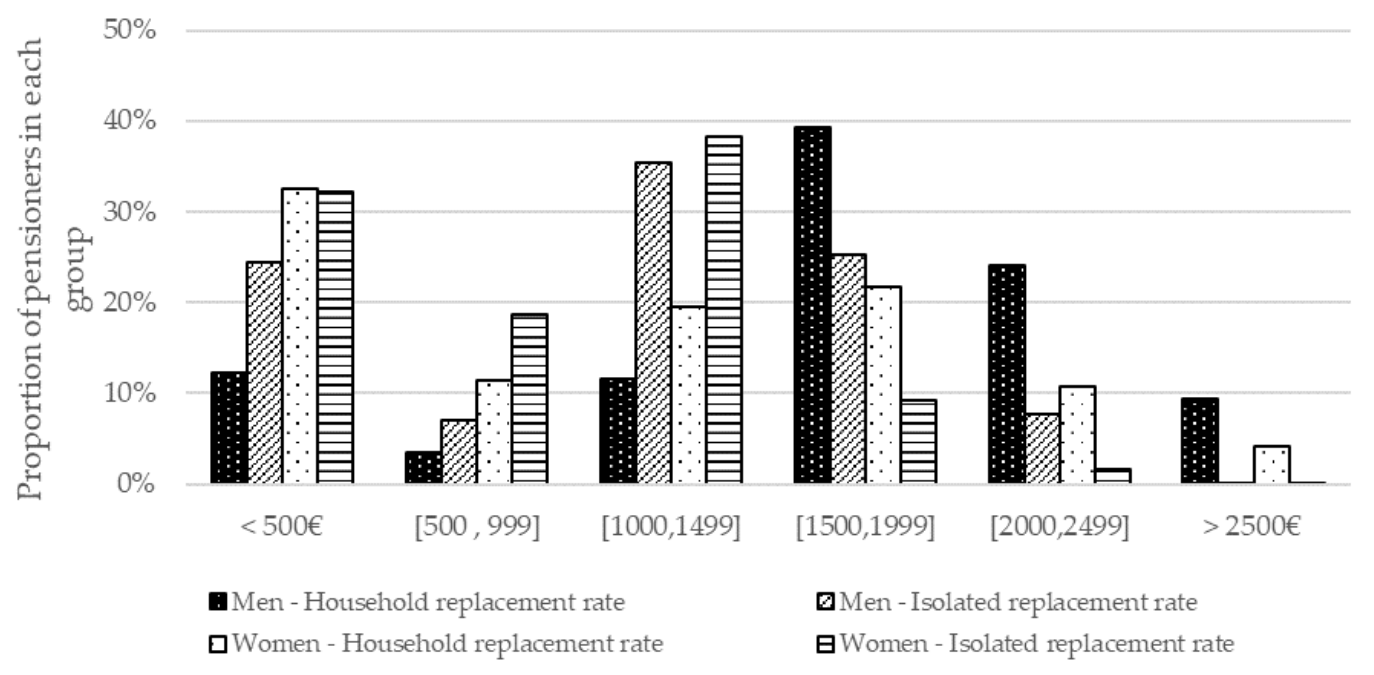

Source: National Pension Office annual reports (1992-2019) 
As a consequence of societal evolutions ${ }^{228}$, sustainability pressures and because of the program's supposed work disincentive effect for dependent spouses, the Belgian Pension Reform Committee recommended removing the household replacement rate for individuals in one-earner households. Nonetheless, due to their enduring role as an important source of income for many poor households, a (long) transition period for minimum pensions is also recommended.

Two arguments work in favour of keeping the household replacement rate in place. First, although pensioners in one-earner households have higher average pension benefits because of the more generous household replacement rate (see figure 6.3), households with an unequal distribution of earnings capacity generally have lower average income and are often concentrated in low qualified jobs with low wages (Pension Reform Committee 2020-2040 (2014), Hindriks (2014)).

Secondly, while the trends in old-age pension benefits receipt confirm a decrease in the traditional male breadwinner household model (see figure 6.2), Höhn, Avramov, and Kotowska (2008) state that we are actually witnessing the rise of a modernized male breadwinner model, in which the husband remains the main earner and the wife often has an incomplete career because of household and care responsibilities. ${ }^{229}$ Similarly, Ciccia and Kotowska (2014) observe that childcare remains the responsibility of women across Europe and public child care services still generally assume a generalized traditional male breadwinner model. In fact, there still exists a vast disparity in terms of old-age pension benefits between men and women that are driven by the gender wage

\footnotetext{
${ }^{228}$ Rise of the two-earner household model, increasing number of divorcees, rise in legal cohabitation practices.

${ }^{229}$ In fact, Höhn et. al. (2008) mention that the modernized male breadwinner household model is more common than the two-earner household model in the Netherlands and is the second most common model, after the two-earner model, in Northern Belgium. Additionally, Mínguez (2004) reports that the traditional male breadwinner model is persistent in most of Southern Europe.
} 
gap, longer life expectancy ${ }^{230}$, shorter careers ${ }^{231}$ and a higher prevalence of part-time work $^{232}$, which are partly the result of household and care activities (Hindriks, 2015). ${ }^{233}$

\subsubsection{RETIREMENT INCENTIVES AND FINANCIALLY DEPENDENT SPOUSE}

In this subsection, we present a brief overview of the literature on the impact of having a financially dependent spouse on retirement incentives. We divide this impact into two effects: (I) the effect of spousal characteristics on retirement incentives and (II) the effect of additional benefits granted on the basis of having a dependent spouse.

\subsubsection{SPOUSAL CHARACTERISTICS}

A person's retirement incentives are influenced not only by individual determinants but also by the characteristics of his or her partner such as age, income and activity status (Coile, 2015). Denaeghel, Mortelmans, and Borghgraef (2011) find that spousal characteristics influence retirement decisions but that individual determinants are stronger while Coile (2004) notes that women are as responsive to their own retirement incentives as men. Coile (2015) mentions that there exist three sources that cause the retirement decision of an individual to be influenced by his or her spouse: (i) common financial resources, (ii) similar preferences (i.e. time preferences) and (iii)

\footnotetext{
${ }^{230}$ The OECD (2020) predicts an average of 25.5 years in retirement for women and 21.1 years in retirement for men.

${ }^{231}$ In 2019, the predicted duration of working life for Belgian men was 35.4 years and 31.6 years for Belgian women (Eurostat, 2020).

${ }^{232}$ On average 42 percent of Belgian women and 9 percent of Belgian men aged 15 to 64 were working parttime between 2004 and 2019, compared to 31.2 and 7.9 percent in Europe, respectively (Eurostat, 2020). Likewise, in chapter four, we find that 68.7 (67.2) percent of working wage-earner women aged 55 to 59 (60 to 64) were working part-time from 2004 to 2010.

${ }^{233}$ In fact, 38.3 percent of female pensioners receive a pension benefit of less than 1,000 euros compared to only 27.1 percent of male pensioners. Only 0.1 percent of female pensioners receive a pension benefit of more than 2,500 euros, compared to 2.6 percent of male pensioners (OECD, 2016).
} 
complementarities in leisure (an individual will enjoy leisure even more once his or her spouse is retired).

Furthermore, both Coile (2004) and Baker (2002) find that there exists a spillover effect of the spouse's retirement incentives on the individual's own retirement behavior, caused by complementarities of leisure and joint decision-making. Coile (2004) finds that the inactivity status of the partner has a positive impact on the retirement probability of individuals because of complementarities of leisure. Similarly, Blau (1998) mentions that a spouse values retirement more once the other spouse is retired and both Blau (1998) and Gustman and Steinmeier (2000) find that a husband is more strongly influenced by having a retired spouse than a wife is. Finally, both spouses plan their joint consumption over their life cycle and try to even out their consumption in the best way possible through cooperative retirement decisions (Gustman and Steinmeier, 2000).

\subsubsection{THE INFLUENCE OF THE HOUSEHOLD REPLACEMENT RATE BENEFITS}

Inside the vast strand of the literature that looks at the effect of social security provisions on the retirement decision ${ }^{234}$, a smaller part of the literature looks at the impact of programs that provide (in)direct benefits to the financially dependent spouse. Blau (1998) uses data from the Retirement History Survey and a discrete time choice model to look at the impact of the US dependent spouse benefits on the retirement behavior of older married couples. He predicts that dependent spouse benefits have a small positive impact on the working probability of husbands and a small negative impact on the working probability of wives. Michaud (2003) provides evidence of the same work (dis)incentive mechanism and finds that the overall effect of the program on labor force participation is

\footnotetext{
${ }^{234}$ See Stock and Wise (1990a), Stock and Wise (1990b), Gruber and Wise (1999), Gruber and Wise (2004) Börsch-Supan and Coile (2020), among others
} 
negative. Knapp (2014) uses a life-cycle model of household savings, labor supply and benefit claiming decisions on the 1992 Health and Retirement study US data and confirms that the US dependent spouse benefits program creates a work disincentive for the low income earner in the couple (usually the wife) and a work incentive for the high income earner (usually the husband). For the prime earner in the couple, this implies that the substitution effect (increased returns to work lead to lower demand for retirement) is more important than the income effect (higher benefits induce a higher demand for retirement). In fact, he tests the impact of abolishing the spouse and survivor benefits and finds that it would increase the labor force participation of wives by 1.27 years and decrease the labor force participation of husbands by 0.53 years. Michaud and Vermeulen (2004) find almost no impact of the elimination of the dependent spouse benefits on labor supply at older ages, although they indicate that there exists a work disincentive effect associated with the program. Finally, Baker (2002) analyses the effect of the introduction of the spouse's allowance in 1975 in Canada and finds that it led to a decrease in the work incentives for both men and women because of the means-tested nature of the benefits.

\subsection{EMPIRICAL STRATEGY}

In this section, we first describe the dataset used in our analysis and our sample selection method. Then, we discuss our pension benefits simulation tool and its validation. Further, we define our classification method of households into one and two-earner types and we provide descriptive statistics of the final sample.

\subsubsection{THE DATASET}

In this chapter, we use the Survey of Health, Ageing and Retirement in Europe (SHARE) dataset. SHARE is a survey dataset that contains panel micro data on health, socioeconomic and family network of individuals aged 50 and over. It is a cross-national 
project that covers 27 European countries and Israel. The data is collected biannually and is organized into waves from wave 1 collected in 2004/2005 to wave 7 collected in 2017 . The target population of the survey is persons aged 50 and over at the time of the sampling who have their regular domicile in the country. ${ }^{235}$ Each wave follows the same baseline sample (born in 1954 in the case of Belgium) and adds a refreshment sample 236 in order to ensure the representation of younger cohorts and to compensate for attrition.

We use the Job Episode panel dataset of waves 3 and 7 for Belgian respondents, which contains working life and employment histories for 6,200 individuals (2,865 in wave 3 and 3,333 in wave 7). ${ }^{237}$ This dataset includes a retrospective career and earnings history that was collected in 2008 or 2009 for wave 3 respondents and in 2017 for wave 7 respondents. The data also includes personal characteristics such as gender, age, a variable indicating whether the individual lives with a partner, the marital status, the occupation (in education, working, retired, unemployed, sick, homemaker, other ${ }^{238}$ ) and the year of birth. Available career information includes the first and last net wage of each new employment, the industry, the working regime (wage earner, self-employed or civil servant) and the working hours (full or part-time). The first old-age pension net amount received is also available if the individual was retired at the time of the survey. The retrospective career and earnings history of the partner is included for part of the sample.

\footnotetext{
235 The sampling design also includes stratification based on the provinces and clustering based on municipalities. Individuals are excluded from the target population if they are incarcerated, hospitalized, cannot be located or unable to speak the national language.

236 The refreshment sample is composed of people born in 1956 in wave 2, 1960 in wave 4, 1962 in wave 5 ad 1964 in wave 6 . No refreshment sample was included in waves 3 and 7.

${ }^{237}$ The job panel dataset is only available for waves 3 and 7 . In case one individual is identified in both waves 3 and 7, we use only the information contained in wave 7 .

238 The other category includes individuals in training, travelling, volunteering or benefitting from a survivor pension only.
} 


\subsubsection{SAMPLING METHODOLOGY}

Because we are working with survey data, we first need to make several adjustments to the initial dataset that contains the life history of individuals. First, we discard information on years spent in education. Moreover, we dispose of individuals who do not report any wage ${ }^{239}$ at any point in their adult life and individuals with a reported spouse who did not participate in the survey. We drop the observations of individuals who report a monthly gross income or pension higher than 10,000 euros as we consider them as outliers or encoding errors.

We keep the observations of individuals between the ages of 55 and $65^{240}$, who were aged younger than 55 in $1990^{241}$ and those who were still working at age 55 . We drop the observations of individuals after they retire because we consider retirement to be an absorptive state. We are mostly interested in individuals who have a wage-earner career because social security rules for the other two working regimes vary considerably in terms of benefits calculation rules, pensionable wage floors, ceilings, etc. ${ }^{242}$ Therefore, we discard the observations of individuals who have a wage-earner career of less than 75 percent $^{243}$ of total career or individuals who are identified as civil servants or selfemployed workers after age 55. Furthermore, we drop individuals who have an estimated

\footnotetext{
${ }^{239}$ Because we are working with retrospective data, we are missing the career and earnings histories of some individuals who refuse to give this information or simply cannot remember.

${ }^{240}$ We follow the same methodology of the previous chapters where we consider the age range of 55 and 65 as the range during which a worker is most probable to exit the labor force. Indeed, most early labor force exits pathways are not available before age 55 and wage-earners are encouraged to retire at 65 .

${ }^{241}$ We restrict the period of our analysis to limit the survivor bias. Indeed, only individuals who have survived until 2008/2009 (wave 3) or 2017 (wave 7) have replied to the survey. As we expect that early death is correlated with health and socio-economic status, the longer the period between the last observation and the time of the survey, the higher the survivor bias.

242 Besides, no household replacement rate exists in the civil servant scheme.

${ }^{243}$ We tested the robustness of our analysis by sampling only individuals with a 100 percent career as a wage-earner. It did not significantly alter our results but it decreased considerably the size of our sample. Therefore, in order to increase the sample size, we allow for mixed careers up to 25 percent of another working regime as it often happens that individuals start their career in a different working regime.
} 
pension amount of less than 500 euros and individuals who have a total career of less than or equal to 15 years because we assume that the determinants of retirement for individuals with a low wage or low number of career years are potentially very different from those of individuals with a higher wage. Finally, we drop individuals who have an estimated pension higher than 3500 euros, since it is higher than the maximum pension benefit.

We obtain a total of 3,392 observations for 716 individuals and 625 households. Observation years range from 1990 to 2016. Table 6.1 summarizes the main characteristics of sampled individuals. Since we are imposing a minimum career length condition, the proportion of men is higher than the proportion of women in our sample.

Table 6.1: Characteristics of sampled individuals

\begin{tabular}{|c|c|c|}
\hline & Number of individuals & Proportion of total sample \\
\hline Male & 437 & $61.03 \%$ \\
\hline Female & 279 & $38.97 \%$ \\
\hline French speaking & 269 & $37.57 \%$ \\
\hline Dutch speaking & 447 & $62.43 \%$ \\
\hline Without partner & 176 & $24.58 \%$ \\
\hline With partner & 540 & $75.42 \%$ \\
\hline Total & 716 & $100 \%$ \\
\hline
\end{tabular}

\subsubsection{RECONSTRUCTION OF EARNINGS HISTORIES AND CALCULATION OF PENSION BENEFITS}

We use the earnings histories that contain the first net wage of each new job to reconstruct complete earnings histories. From these reconstructed earnings histories, we estimate the old-age pension benefits each individual would be entitled to receive at each age between 55 and 65 . We detail the method for building our old-age pension benefit calculator below. 
First, we transform the currency of wages into euros and we calculate gross wages from net amounts using the respective taxation rules in effect. ${ }^{249}$ We fill-in the partial wage information by adjusting the first wage of each new employment for inflation and average wage growth ${ }^{244}$ for each year of career. In case there is missing information on the first wage of a new job, we use the closest available wage in the individual's earnings history, adjusted for inflation and wage growth.

We use these reconstructed earnings histories to calculate the old-age pension benefits entitlements at each age between 55 and 65. First, we replace the wage with the corresponding pensionable earnings ceiling (floor) ${ }^{245}$, if the former is larger (smaller) than the latter. Second, we reevaluate past earnings into euros of the retirement year using the corresponding valorisation factor. ${ }^{246}$ Finally, we sum the valorized earnings over the entire career, divide the sum by the applicable complete career years ${ }^{247}$ and multiply it by 60 percent for individuals in two-earner or single households and 75 percent for individuals in one-earner households (see section 6.3.5). We replace our estimated oldage pension amount by the minimum pension where needed, adjusted for the proportion of a complete career. ${ }^{248}$ Finally, we calculate the net pension amount using the respective taxation rules in effect ${ }^{249}$ and we add the pension bonus whenever applicable. ${ }^{250}$ For each age between 55 and 65, we obtain an estimation of the net pension amount an individual

\footnotetext{
${ }^{244}$ We adjust wages to inflation using the historical evolution of Consumer Price Index. For wage growth, we use the historical evolution of average monthly salary for wage earners by age, which is differentiated by gender. See the appendix for the evolution of average wage growth.

${ }^{245}$ See chapter two for a list of pensionable earnings ceilings and floors.

${ }^{246}$ See chapter two for more detail on the valorisation factor.

${ }^{247}$ The complete career years in the old-age pension calculation are 45 years for men. For women they are 40 years before 1997, 41 years between 1997 and 1999, 42 years between 2000 and 2002, 43 years between 2003 and 2005, 44 years between 2006 and 2008 and 45 years from 2009 onwards. See chapter two for more information on complete career years.

${ }^{248}$ See chapter two for more information on minimum pensions.

249 See chapter two for a historical evolution of taxation rules.

250 See chapter two for more information on the pension bonus.
} 
would be entitled to receive based on his reconstructed earnings history, were he to retire at that age..$^{251}$

We find an average estimated old-age pension of 1329.92 euros for men and 1074.17 euros for women (all years combined, in 2017 euros). Figure 6.4 displays the evolution of oldage pension benefits by age and gender. ${ }^{252}$

Figure 6.4: Evolution of estimated old-age pension amounts by age and gender (2017 constant euros)

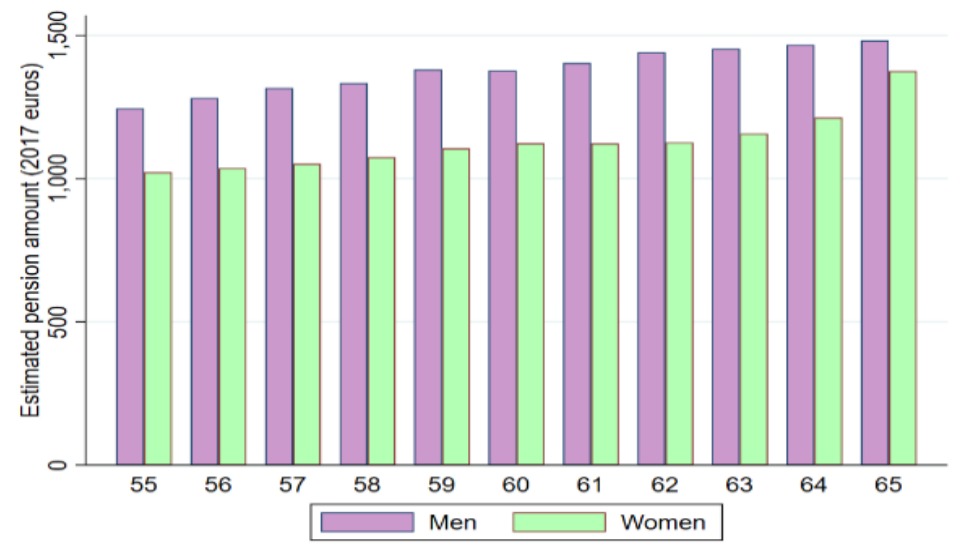

Source: Authors' own calculations using SHARE dataset

\subsubsection{VALIDATION OF THE OLD-AGE PENSION BENEFITS CALCULATOR}

For each sampled individual, we compare our estimated pension amount at the effective retirement age to the corresponding observed amounts received, as reported in later survey waves (see section 6.3.1). ${ }^{253}$ We find a difference between the observed and the

\footnotetext{
${ }^{251}$ We convert every pension amount into 2017 euros using the Consumer Price Index.

${ }^{252}$ The important increase in average pension benefits at age 64 and 65 for women is caused by the fact that individuals with the highest earnings tend to stay on the labor force after the statutory eligibility age. ${ }^{253}$ We restrict the sample to individuals who were retired at the time of the survey (to have information on his or her observed old-age pension) and who never worked as self-employed or civil servants. If the old-age pension amount is not observable at the retirement age, we adjust it to the price levels of the retirement year using the pivotal index mechanism (see chapter two).
} 
estimated pension amount of less than 250 euros for 50.2 percent of our observations and less than 500 euros for 79.8 percent of our observations.

Figure 6.5 compares the real and estimated pension amounts by age for men and women. The estimation is quite accurate for men but less so for women. First, women more often have interrupted careers or part-time working contracts because of household and care responsibilities. For the same reasons, women exhibit more variations in wage growth and in earnings paths than men. Second, since we do not have precise information on work intensity during a specific career year ${ }^{254}$, we cannot truly verify the eligibility criteria for the minimum pension and we may grant it to individuals who would actually not be eligible based on their real career histories. This missing information thus has a stronger effect on the estimates for women for whom part-time work is much more prevalent (see chapter five). In fact, if we run a regression of the absolute difference between the observed and the estimated pension amount, using various personal and job-related characteristics as explanatory variables, we observe that gender and the education level (tertiary compared to secondary) both have a positive and significant coefficient. This result indicates that the average wage growth we use for the reconstruction of earnings histories is not as appropriate for women and individuals with tertiary education as it is for other types of individuals. Consequently, our estimation of financial retirement incentives is less accurate for women and individuals with tertiary education than other types of individuals.

\footnotetext{
${ }^{254}$ We have information on whether the individual has worked part-time during the year but nothing on how many days or full-time equivalent he or she has worked during that year.
} 
Figure 6.5: Estimated and observed old-age pension benefits by gender
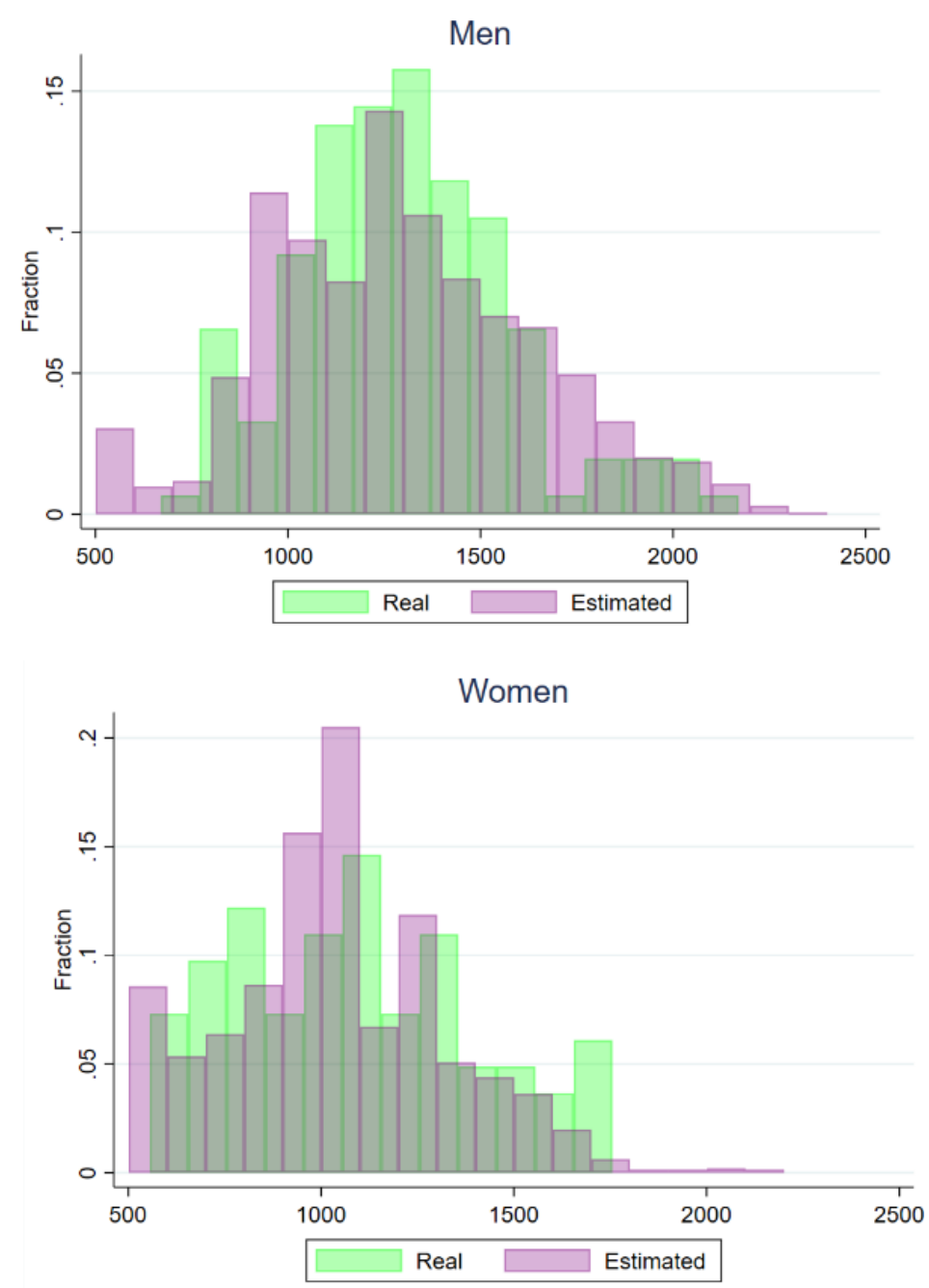

Source: Authors' own calculations using SHARE datasets - Modules Job panel (waves 3 and 7) and Employment and pensions (waves 1, 2, 3, 4, 5, 6)

\subsubsection{CLASSIFICATION INTO ONE AND TWO-EARNER HOUSEHOLDS}

In order to be identified as a one-earner household, the couple must be married and the household must include one earner and one financially dependent spouse. We classify an individual as a financial dependent spouse using two criteria: a status-based indicator and an earnings indicator. The status indicator labels an individual as a financially dependent spouse if he or she is identified as homemaker, in training or "doing nothing". 
The earnings indicator compares the individual's earnings (from any of the three working regimes) to the corresponding earnings test thresholds ${ }^{255}$. We exclude individuals who qualify based on the earnings indicator but are identified as sick or unemployed because the household replacement rate cannot be granted to a pensioner whose spouse receives unemployment or sickness benefits. Since the household replacement rate is only available to married individuals, we exclude legal cohabitants from this definition. ${ }^{256}$ Households with one spouse identified as a financial dependent using either of the two indicators is labelled a one-earner household. ${ }^{257}$ Households with two spouses working and earning an income above the wage indicator are labelled two-earner households and one person households are labelled single households. Finally, we only keep the observations of the earners in one-earner couples and discard the observations of the dependent spouse.

We obtain a total of 129 individuals (760 observations) in one-earner households, 319 individuals (1,820 observations) in two-earners households ${ }^{258}$ and 144 individuals (812 observations) in single households. Table 6.2 summarizes the main characteristics of individuals by type of household. Predictably, there are more men identified as earners in one-earner households following the prevalence of the traditional male breadwinner model. The proportion of men in two-earner couples is also slightly higher than for women because of our sampling methodology that discards individuals with (very) short

\footnotetext{
255 We use the 2017 earnings threshold that we adjust for prices. The monthly threshold (in 2017 euros) is 1,013.5 euros for wage-earners or civil servants below age 65, 810.7 euros for self-employed workers below age $65,1,971.4$ euros for wage-earners or civil servants above age 65 or 1,899.1 euros for selfemployed workers above age 65 . These thresholds correspond to the earnings test for the combination of work and old age pension benefits receipt.

256 Unmarried individuals who satisfy the one earner household criteria are not used in this analysis. They only constitute a very minor proportion of the sample.

${ }^{257}$ If both household members fulfil the financially dependent spouse criteria, then the observations are dropped.

${ }^{258}$ It is possible that two individuals from the same two-earner household are selected into our sample.
} 
careers or who are out of the labor force at older ages. Interestingly, we note that the average retirement age is almost equal between single and two-earner households but is lower for one-earner households. Finally, we observe that there is a lower prevalence of part-time work among individuals in one-earner households compared to other types of households.

Table 6.2: Main characteristics of sampled individuals by type of household

\begin{tabular}{|c|c|c|c|c|c|c|}
\hline \multirow[b]{2}{*}{ Personal characteristics } & \multicolumn{2}{|c|}{ One-earner } & \multicolumn{2}{|c|}{ Two-earners } & \multicolumn{2}{|c|}{ Single } \\
\hline & $\begin{array}{l}\text { Number of } \\
\text { individuals }\end{array}$ & $\%$ & $\begin{array}{l}\text { Number of } \\
\text { individuals }\end{array}$ & $\%$ & $\begin{array}{l}\text { Number of } \\
\text { individuals } \\
\end{array}$ & $\%$ \\
\hline Total (individuals) & 129 & 100 & 319 & 100 & 144 & 100 \\
\hline \multicolumn{7}{|l|}{ Gender } \\
\hline Male & 113 & 87.6 & 176 & 55.17 & 77 & 53.47 \\
\hline Female & 16 & 12.4 & 143 & 44.83 & 67 & 46.53 \\
\hline \multicolumn{7}{|l|}{ Education } \\
\hline Primary & 10 & 7.75 & 28 & 8.78 & 10 & 6.94 \\
\hline Secondary & 65 & 50.39 & 170 & 53.29 & 74 & 51.39 \\
\hline Tertiary & 54 & 41.86 & 121 & 37.93 & 60 & 41.67 \\
\hline \multicolumn{7}{|l|}{ Native language } \\
\hline French & 43 & 33.33 & 122 & 38.24 & 57 & 39.58 \\
\hline Dutch & 86 & 66.67 & 197 & 61.76 & 87 & 60.42 \\
\hline Average retirement age & \multicolumn{2}{|c|}{$60.98(2.81)$} & \multicolumn{2}{|c|}{$61.16(2.93)$} & \multicolumn{2}{|c|}{$61.82(2.79)$} \\
\hline
\end{tabular}




\begin{tabular}{|c|c|c|c|c|c|c|}
\hline \multirow[b]{2}{*}{ Job-related characteristics } & \multicolumn{2}{|c|}{ One-earner } & \multicolumn{2}{|c|}{ Two-earners } & \multicolumn{2}{|c|}{ Single } \\
\hline & $\begin{array}{c}\text { Number of } \\
\text { observations }\end{array}$ & $\%$ & $\begin{array}{c}\text { Number of } \\
\text { observations }\end{array}$ & $\%$ & $\begin{array}{c}\text { Number of } \\
\text { observations }\end{array}$ & $\%$ \\
\hline Total (observations) & 760 & 100 & 1,820 & 100 & 812 & 100 \\
\hline Part-time work & 48 & 6.32 & 314 & 17.25 & 129 & 15.89 \\
\hline Full-time work & 712 & 93.68 & 1,506 & 82.75 & 683 & 84.11 \\
\hline \multicolumn{7}{|l|}{ Sector of activity } \\
\hline Primary & 30 & 3.95 & 20 & 1.10 & 10 & 1.23 \\
\hline Secondary & 432 & 56.84 & 857 & 47.09 & 295 & 36.33 \\
\hline Tertiary & 298 & 39.21 & 943 & 51.81 & 507 & 62.44 \\
\hline $\begin{array}{l}\text { Proportion of career worked } \\
\text { as wage-earner }\end{array}$ & \multicolumn{2}{|c|}{$97.80(4.64)$} & \multicolumn{2}{|c|}{$97.77(5.16)$} & \multicolumn{2}{|c|}{$97.24(6.07)$} \\
\hline
\end{tabular}

Source: Authors' own calculations using SHARE dataset

Looking at the retirement hazard rates (figure 6.6), we see that the retirement hazard of individuals in single households is generally lower than other types of households at lower ages and present peaks at ages 60,64 and 65. The retirement hazard rate of individuals in one-earner households is generally higher than other types of households before age 62 . The only two exceptions are at ages 58 and 60 , the eligibility ages for the conventional early retirement and the early eligibility age of the old-age pension benefit, at which the retirement hazard rates of individuals in two-earner households are the highest. At age 65, the retirement hazard rate of two-earner households is the highest but is closely followed by the other two types of households. 
Figure 6.6: Retirement hazard rates by age and type of household

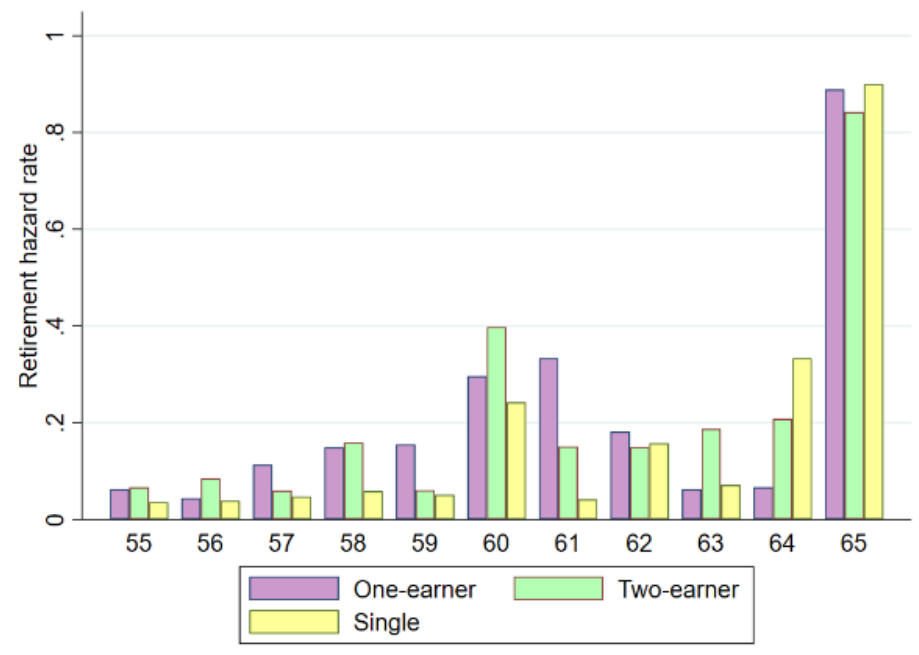

Source: Author' own calculations using SHARE dataset

\subsubsection{FINANCIAL RETIREMENT INCENTIVES}

From the estimated old-age pension benefits, we construct two measures of financial retirement incentives at the individual level: the social security wealth measure and the accrual. We define social security wealth (SSW) as the present discounted value of all future pension benefit ${ }^{259}$ flows for individual $\mathrm{i}$ at age $\mathrm{a}$ if he retires at age $\mathrm{R}^{260}$.

$$
\operatorname{SSW}(i, a, R)=\sum_{s=R}^{T} \beta^{s-a} E\left[B_{i R}(s)\right]
$$

\footnotetext{
${ }^{259}$ Departing form the methodology of chapter four, we only consider the incentives stemming from the old-age pension program and no benefits from other labor force exit pathways because we have access to less detailed status information and we cannot verify most of the eligibility criteria for other programs. In fact, individuals who are marked as receiving disability, conventional early retirement or unemployment insurance after the age of 54 are not included in the sample (see section 3.4). Also, due to a lack of precise information on the individual's wage, we do not construct the ITAX measure.

${ }^{260}$ We assume that the individual retires as soon as benefits become accessible.
} 
where $E\left[B_{R}(s)\right]$ is the expected after-tax benefit of individual $\mathrm{i}$ at age $\mathrm{s}$ for retirement at age $\mathrm{R}$ and received until the end of life T. Discounting is done allowing for time preference $\beta^{s-a}$ is the time discount rate that we assume to be equal to 3 percent real.

We follow Jousten and Tarentchenko (2014) and calculate the expected benefit as

$$
\begin{aligned}
& E\left[B_{R}(s)\right] \\
& =\left\{\begin{array}{c}
\rho(s) B_{R, \text { isolated }}(s) \quad \text { for singles } \\
\rho(s) \tau(s) B_{R, \text { single }}(s)+\rho(s)(1-\tau(s)) B_{R, \text { isolated }}(s) \\
+(1-\rho(s)) \tau(s) B_{R, \text { survivor }}(s) \text { for two }- \text { earner } h \text { h } s \\
\rho(s) \tau(s) B_{R, \text { household }}(s)+\rho(s)(1-\tau(s)) B_{R, \text { isolated }}(s) \\
+(1-\rho(s)) \tau(s) B_{R, \text { survivor }}(s) \text { for one }- \text { earner hhs }
\end{array}\right.
\end{aligned}
$$

Where $B_{R, \text { isolated }}(s)$ refers to the old-age pension benefit paid at the isolated replacement rate at age $s$ if the worker retires at age R. $B_{R, \text { household }}(s)$ refers to the old-age pension benefit paid at the household replacement rate at age $s$ if the worker retires at age $R$. $B_{R, \text { survivor }}(s)$ refers to the survivor pension paid to the spouse if the worker retires at age $\mathrm{R}$ and in case of the worker's passing ${ }^{261} . \rho(s)$ refers to the survival probability of the reference person at age s conditional on being alive at age a, and $\tau(s)$ refers to the survival probability of the spouse at age s conditional on being alive at age a. ${ }^{262}$

\footnotetext{
261 See chapter two for a detailed description of the survivor pension.

${ }^{262}$ The survival probabilities are based on age and gender specific survival tables retrieved from the Human Mortality Database and are similar to the ones used in chapters three and four. We assume that both spouses are the same age.
} 
If the individual is not eligible for the old-age pension benefit at age a, then we compute his social security wealth by imputing an income of zero until he or she becomes eligible for old-age pension benefits at age $\mathrm{R}^{260}$. If the individual is eligible for old-age pension benefits at age a, then a is equal to R and we compute his SSW by imputing pension benefits starting at age a.

Figure 6.7 shows the evolution of social security wealth by age. Similarly to the analysis in chapter three and 4, an individual's social security wealth increases until the individual becomes eligible for the old-age pension benefit (age 60 in most cases) and then slightly decreases because the additional pension wealth gained through increased pension benefits is lower than the wealth lost because the individual foregoes one year of benefits (see chapter four).

Figure 6.7: Evolution of social security wealth by age

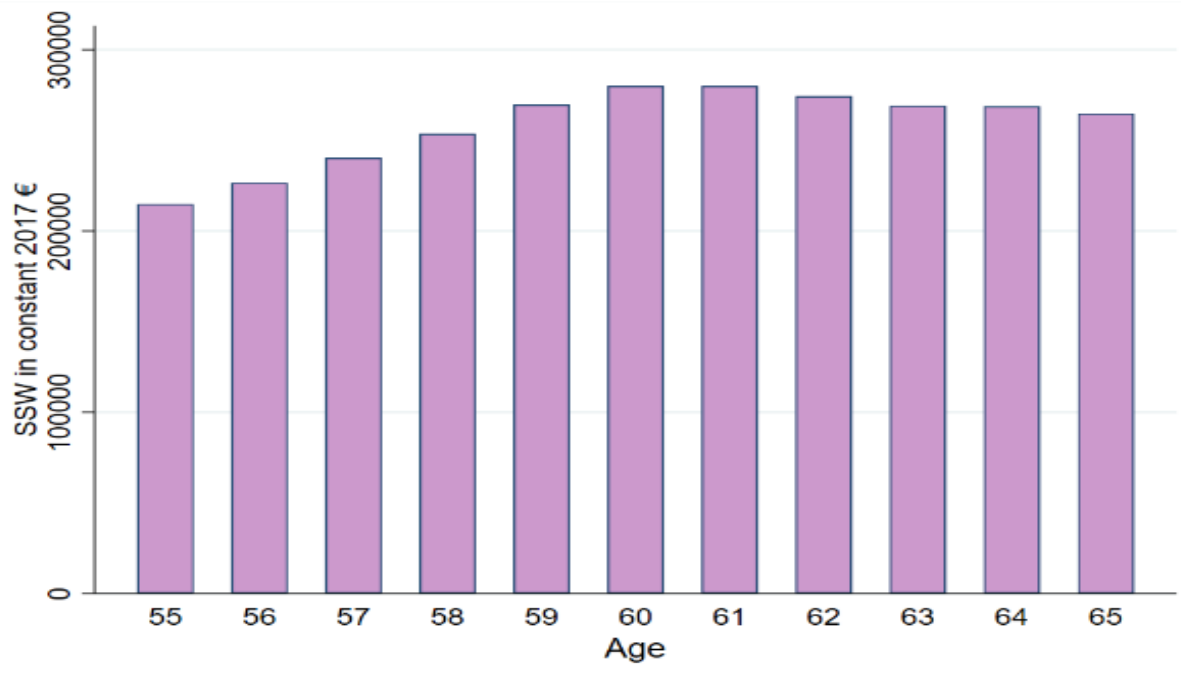

Source: Authors' own calculations using SHARE dataset

The accrual represents the variation in SSW that is obtained if the worker stays on the labor force for one additional year. A positive accrual represents an incentive to stay on the labor force because the pension wealth to be gained by working for one more year is 
higher than the effect of losing one year of benefits. In the opposite case, a negative accrual represents an incentive to leave the labor force.

$$
A C C_{t}(i, a)=S S W_{t+1}(i, a+1)-S S W_{t}(i, a)
$$

Figure 6.8 shows the evolution of accruals by age. Similarly to chapter three and 4 and to the literature (see Gruber and Wise, 1999), we find large positive accruals before benefits become available and smaller accruals thereafter. The accruals before age 60 (the early eligibility age of the old-age pension) are positive and thus indicate that there is an incentive for the individual to stay on the labor market before he or she can access oldage pension benefits.

Compared to the social security wealth and accrual measures presented in chapter four, these financial retirement incentives were calculated based on incomplete earnings histories that have been reconstructed assuming a constant and continuous increase in wages every year. Therefore, the longer an individual stays on the labor force before he is eligible for the early eligibility age of old-age pension, the higher the accrual because on top of the increasing conditional survival probability until old-age pension becomes accessible, the amount of pension benefits he is entitled to increases as well. This explains why the accruals increase between age 55 and 59. After age 60 , the accruals turn negative because old-age pension benefits becomes accessible for most sampled individuals and the effect of losing one year of old-age benefit is larger than the increase in social security wealth following on additional year of career. 
Figure 6.8: Evolution of accruals by age

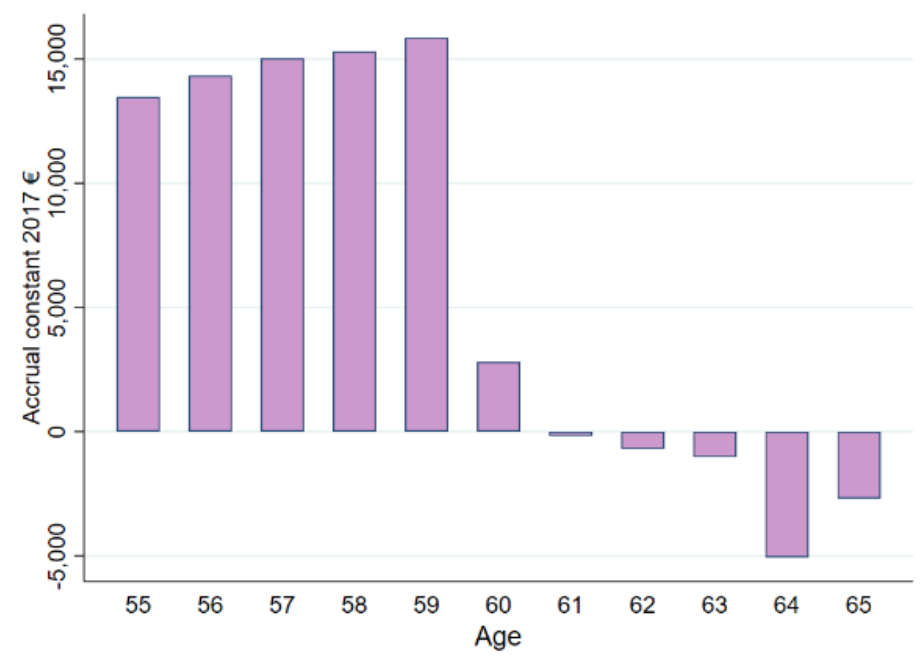

Source: Authors' own calculations using SHARE dataset

\subsection{ANALYSIS}

In this section, we first define the model we use in our analysis, namely a discrete time logistic duration model. Then, we discuss censoring and truncation issues that arise with our data and which are common in duration model analysis. Finally, we define the explanatory variables we include in our analysis.

\subsubsection{THE MODEL}

We use a discrete time ${ }^{263}$ duration model to study transitions from employment into retirement. ${ }^{264}$ The use of such framework allows us to model the conditional probability

\footnotetext{
${ }^{263}$ Retirement occurs on a continuous timeline but since our data is grouped into annual intervals, discrete time duration models are more appropriate than continuous time duration models (Jenkins, 2004). When one has access to intervals grouped in months, continuous time duration models can still be used to analyse the retirement decision (see Aranki and Macchiarelli (2013)) for instance).

264 See Diamond-Hausman (1984), Schils (2006), Wolthoff, Euwas and Vuuren (2006), Euwals- and Vanvuuren- and Wolthoff(2006), Lindeboom (1998), Antolin and Scarpetta (1998) and Aranki and Macchiarelli (2013) for duration models of retirement transitions.
} 
that the employment spell will end during a certain period, given that it has lasted until the preceding period.

We refer to the transition from employment to retirement as a failure event, which can only occur after the onset of risk, the individual's first entry on the labor market. Failure can only happen once since we define the retirement decision as absorptive. We define $\mathrm{T}$ a discrete random variable that represents employment duration. Our time axis is divided into a number of non-overlapping continuous time intervals where the boundaries are the employment duration years $a_{0}, a_{1}, a_{2}, a_{3}, \ldots, a_{k}$. Thus, the intervals are defined as $\left[a_{j-1}, a_{j}[\right.$ with $j \in\{1,2,3, \ldots k\}$.

The survival function of interval $a_{j}, \mathrm{~S}\left(a_{j}\right)$, is the probability that the employment spell will last until at least $a_{j}$. Said differently, it is the probability that no retirement occurs before $a_{j}$. The failure function, $\mathrm{F}\left(a_{j}\right)$, is the reverse of $\mathrm{S}\left(a_{j}\right)$ and is the probability of retirement before or during interval $a_{j}$.

$$
\begin{gathered}
S\left(a_{j-1}\right)=\operatorname{Pr}\left[T>a_{j-1}\right]=1-\mathrm{F}\left(a_{j-1}\right) \\
S\left(a_{j}\right)=\operatorname{Pr}\left[T>a_{j}\right]=1-\mathrm{F}\left(a_{j}\right)
\end{gathered}
$$

The probability of retirement during the interval $\left[a_{j-1}, a_{j}[\right.$ is written as

$$
\operatorname{Pr}\left[a_{j-1}<T \leq a_{j}\right]=\mathrm{F}\left(a_{j}\right)-\mathrm{F}\left(a_{j-1}\right)=S\left(a_{j-1}\right)-\mathrm{S}\left(a_{j}\right)
$$


The discrete time hazard rate at interval $a_{j}, h\left(a_{j}\right)$, is the conditional probability of retirement during interval $\left[a_{j-1}, a_{j}[\right.$, provided the individual was still employed at the end of interval $a_{j-1}$, and is written as

$$
h\left(a_{j}\right)=\operatorname{Pr}\left[a_{j-1}<T \leq a_{j} \mid T>a_{j-1}\right]
$$

We follow the methodology of Maes (2008) and Andriopoulou and Tsakoglou (2011) and we use a logistic discrete time hazard duration model in which the dependent variable is the logit transformation of the hazard rate at interval $j^{265}$ (see 5). ${ }^{266}$

In the logistic model, the log of the relative odds ${ }^{267}$ of failure during interval $\mathrm{j}$, conditional upon having survived until the end of the interval ( $j-1)$, is the sum of a baseline hazard that is common to every individual and an individual-specific scaling factor.

$$
\begin{gathered}
\operatorname{logit}(h(j, X))=\log \left(\frac{h(j, X)}{1-h(j, X)}\right)=f(j)+\beta^{\prime} X^{\prime} \\
h(j, X)=\frac{1}{1+e^{\left(-f(j)-\beta^{\prime} X^{\prime}\right)}}
\end{gathered}
$$

Where $h(j, X)$ is the scaled hazard rate for a certain individual in interval j. $f(j)=$ $\operatorname{logit}\left(h_{0}(j)\right)$ is the baseline hazard specification, common to every individual. And $X$ is

\footnotetext{
${ }^{265}$ Hereafter, since all intervals have the same length, we refer to interval $a_{j}$ as interval $\mathrm{j}$.

266 There exists two main hazard specification functions in discrete time models: the complementary log$\log$ and the logistic model. The main difference between the two models is that the complementary log$\log$ model assumes proportional hazards and the logistic model assumes proportional odds (Andriopoulou and Tsakloglou, 2011). In fact, the logistic model converges to the complementary log-log model as the hazard becomes increasingly small, which is the case in most applications (Jenkins, 1995). ${ }^{267}$ Therefore, the exponentiated coefficients of the logistic model can be interpreted as odds ratio, or the probability of failure over the probability of non-failure. We follow Maes (2008) and Wolthoff, Euwas and Vuuren (2006) and we present the marginal effects rather than the exponentiated coefficients for the sake of clarity and simplicity of interpreation.
} 
the vector of household, personal and job characteristics for a certain individual, which can be time constant or time-varying.

Because unobserved effects like ability, motivation, general attitudes towards employment or retirement and preferences for leisure might affect the retirement behavior (Maes, 2008), we control for individual heterogeneity using a random effects model where $\mathrm{c}$ is the unobserved individual-specific error term that we assume uncorrelated with vector $\mathrm{X}$.

$$
\operatorname{logit}(h(j, X))=\log \left(\frac{h(j, X)}{1-h(j, X)}\right)=f(j)+\beta^{\prime} X^{\prime}+c
$$

Jenkins $(1995,2004)$ shows that a discrete time duration model with panel data is equivalent to estimating a logit model using the failure event as the dependent variable and a set of explanatory variables representing duration.

Therefore, we use the following binary response logit model

$$
y_{i j}^{*}=\beta^{\prime} X^{\prime}+f(j)+c_{i}+u_{i j}
$$

where $y_{i j}^{*}$ is the latent probability for individual i to retire in interval $j, \beta^{\prime}$ is the vector of coefficients associated with time-constant and time-varying explanatory variables $X^{\prime}$ and $f(j)$ is the baseline hazard specification of employment duration.

\subsubsection{CENSORING AND TRUNCATION}

One of the advantage of duration models lies in the fact that they effectively deal with the presence of right-censored and left-truncated data. Figure 6.9 summarizes the types of censoring and truncation issues we face with our data.

Left truncation is caused by our sampling methodology, according to which we only keep individuals who are still employed at age 55 and leave aside individuals who retired 
before that age. Therefore, our results are not valid for the portion of the population who is allowed to retire at a (very) early age. ${ }^{268}$ Another source of truncation is caused by the fact that we only observe individuals who are still alive at the time of survey. As mentioned above, we restrict our sample to individuals who were aged 55 or less in 1990 and discard the observations of older individuals in order to reduce a potential attrition bias.

Right censoring occurs because not everyone who replied to the survey in wave 3 and wave 7 have retired. Indeed, 28.77 percent of individuals in our initial sample were still employed at the end of our observation period (2008 or 2009 for wave 3 and 2017 for wave 7). We assume that censoring is independent of the probability to retire since it is solely based on the year in which the survey took place. Moreover, because we use a logit random effect model on panel data, we are effectively dealing with censoring issues.

\footnotetext{
${ }^{268}$ For instance, sailors, flying personnel, etc. have different old-age pension eligibility rules and are more likely to retire early. Since they might respond to different retirement incentives based on specific eligibility rules, they are left out of our analysis.
} 
Figure 6.9: Illustration of standard observation, left truncation and right censoring in the data

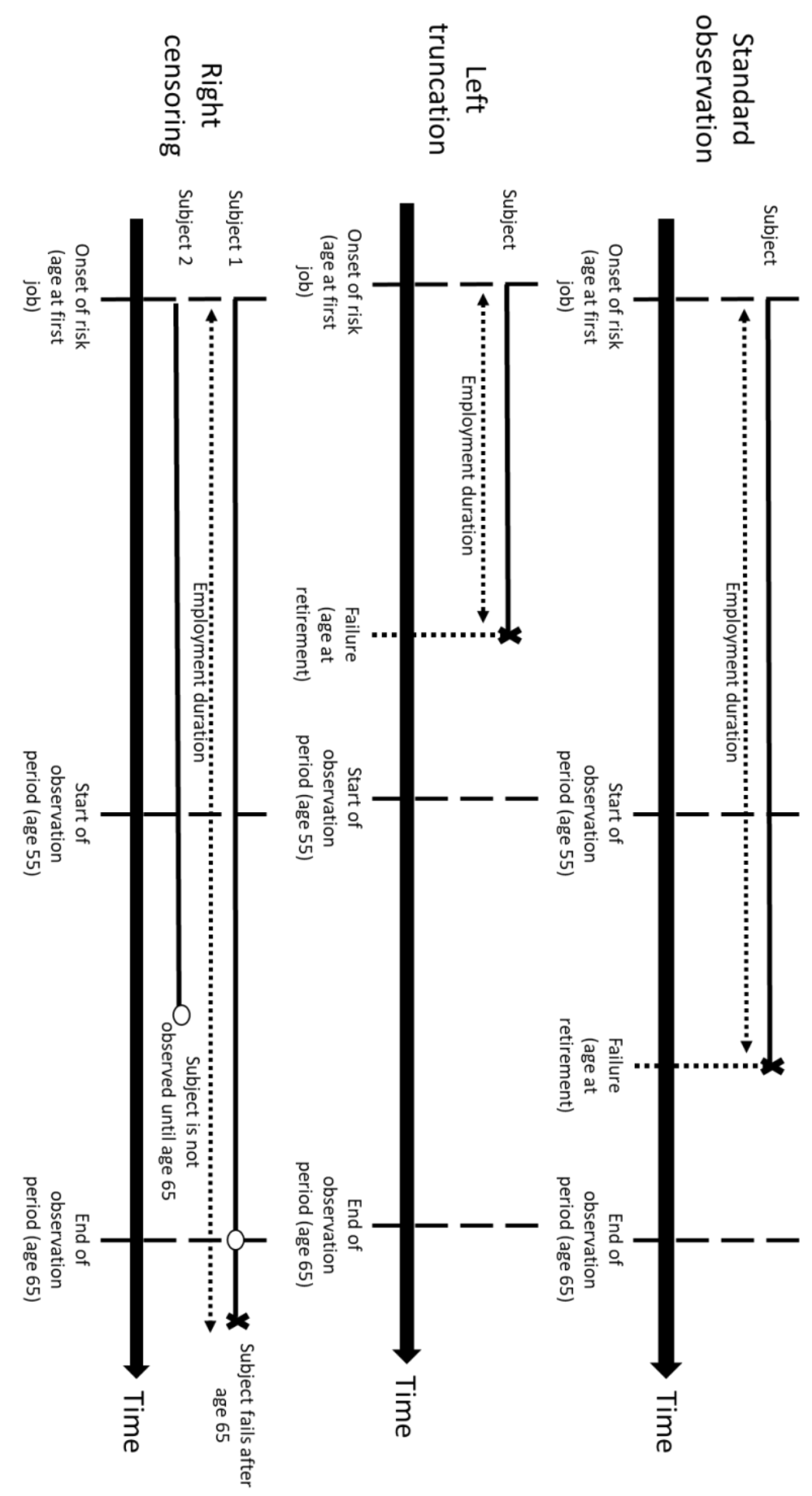

Source: Authors' own illustration 


\subsubsection{DEPENDENT AND EXPLANATORY VARIABLES}

Our dependent variable is a binary indicator of retirement, defined as a transition from employment status to the "retired from work" status ${ }^{269}$ during period interval $\mathrm{j}$.

$$
\text { Retirement }_{i j}=\left\{\begin{array}{c}
1 \text { if working in interval }(j-1) \text { and retired in interval } j \\
0 \text { otherwise }
\end{array}\right.
$$

We use our financial retirement incentive measures, the social security wealth measure and the accrual, as explanatory variables ${ }^{270}$. Maes (2008) mentions the identification issue of social security wealth, according to which social security wealth might capture the unobserved effect of individuals' taste for work. Indeed, individuals with a high taste for work have higher average wages and social security wealth and will likely retire later than individuals with low taste for work. In fact, while the lifecycle theory suggests a positive effect of social security wealth on retirement, this omitted variable bias might lead to a negative coefficient for social security wealth. Coile and Gruber (2001) found that there was still substantial variation in the SSW and accruals even after controlling for lifetime and current earnings. Therefore, following the non-linearity in the relationship between SSW and earnings, we add a variable indicating the last net monthly wage ${ }^{271}$ received by the individual as a proxy for the unobserved effect of taste for work.

In additional to financial retirement incentives, we control for job characteristics including the sector of activity (primary, secondary or tertiary), part-time working contracts (at least one period of part-time work during interval $j$ takes the value of 1 ) and the years worked

\footnotetext{
${ }^{269}$ The status Retired from work indicates the receipt of (part or full-time) old-age pension benefits.

270 One unit change in the social security wealth and accrual variables is equal to a change of 100,000 euros.

${ }^{271}$ A unit change in the net wage variable is equal to a change of 100 euros. The wages are expressed in 2017 euros.
} 
as wage-earner expressed as a percentage of the career ${ }^{272}$. Next, we control for personal and household characteristics including gender, the year of birth ${ }^{273}$, the level of education (primary, secondary or tertiary), an interaction term between education and gender, the language spoken ${ }^{274}$, age difference with the partner (in absolute value), a binary variable indicating whether the spouse is older than the worker ( 1 if the spouse is older than the worker and 0 otherwise) and the retirement status ${ }^{275}$ of the partner ( 1 if the partner is retired from work and 0 otherwise). We add a variable indicating whether the individual is an earner in a one-earner household following our classification method detailed above.

Finally, we add a set of variables representing the baseline hazard function of employment duration. We use a second order polynomial function of employment duration. ${ }^{276}$

$$
f(j)=z_{1} j+z_{2} j^{2}
$$

Duration can be proxied by age itself (Maes (2008), Spataro (2002)), and it can thus become difficult to discern the difference between the effect of the duration variable and the pure age effects. Coile and Gruber (2001) note that they find very little difference in their coefficients when including the age dummies or another specification of age. We follow the methodology of Maes (2008), Meghir-Whitehouse (1997) and Lindeboom (1998) and

\footnotetext{
272 One unit change in this variable is equal to a change of 10 percentage points.

273 Since we merged two waves of the data (wave 3 and 7), we tested for the impact of belonging to one wave or another and have found no significant effect.

${ }^{274}$ French speakers live in a region that broadly corresponds to Wallonia and Flemish speakers live in a region that broadly corresponds to Flanders. The German speaking community is not included in the survey.

275 To be identified as retired, the partner must have had a career. Therefore, not every dependent spouse in one-earner household is defined as retired.

276 We tried various baseline hazard specifications: linear, dummies, dummies of 5 years, log, etc. All specifications yielded very similar results.
} 
include employment duration and an age variable as we expect that individuals with the same employment duration and a different age can have a different retirement behavior.

Regarding age, we use three different model specifications. Indeed, age is plausibly correlated with decreasing health, increasing preferences for leisure, social norms and eligibility conditions for various social security programs (Maes, 2008). We control for the correlation of age with unobservables by using a random effects model. However, age might still capture eligibility for social security programs, which should be captured by our financial incentive variables. We are faced with a dilemma. On the one hand, the use of a set of age dummies serves to capture non-linearity in the effect of age, but it avoids our financial incentive measures from capturing the eligibility effect of social security programs. On the other hand, the use of a non-linear function of age foregoes this latter issue but might not capture the social norms effect of reaching a certain age on the retirement probability. In view of these assumptions, we present three models with different age specifications: (i) a cubic form specification of age, (ii) age dummies and (iii) a cubic form specification of age and a set of social security eligibility variables. Therefore, in the third specification, we add a set of binary variables indicating eligibility to old-age pension at the early (EEA) and statutory (SEA) ages and eligibility to the standard conventional early retirement regime. ${ }^{277}$

\footnotetext{
277 See chapter two for a historical description of the eligibility conditions for these programs. Since we do not have access to the days worked during each year of career, we consider that each year of the career was worked full-time when checking the eligibility criteria.
} 


\subsection{RESULTS}

In this section, we first present and discuss the results of our discrete time logistic duration model. Then, we present a simulation of the impact of a change in the generosity of the household replacement rate on predictions of the retirement probability and on poverty measures.

\subsubsection{REGRESSION RESULTS}

In this section, we present and discuss the results of our discrete time logistic hazard duration model divided into three models with different age and social security eligibility specifications (see table 6.3): (i) a cubic polynomial of age, (ii) age dummies and (iii) a cubic polynomial of age with social security eligibility variables.

First and foremost, we take a look at the effect of our financial retirement incentive variables. Similarly to chapter four, we find a significant and positive impact of social security wealth on retirement in all three models. This result indicates that higher social security wealth leads to higher retirement probability, thus correctly predicting an income effect (Coile, 2004; Maes, 2008). As expected, the effect of our last net wage variable is negative and significant, and represents the negative relationship between taste for work and retirement. Similarly to Gruber and Wise (2004), we observe that part of the social security wealth effect is captured by the wage coefficient as the coefficient of the former decreases as the latter is added as a regressor. In the end, we find that a 100,000 euros increase in social security wealth would increase the retirement probability by approximately 6 percentage points. ${ }^{278}$

\footnotetext{
${ }^{278}$ We find a social security wealth coefficient that is substantially lower than in chapter three. Several causes are at the root of this observation. First, we reconstruct complete earnings histories from partial
} 
The effect of the accrual variable is negative and significant, thus correctly predicting a substitution effect (Coile, 2004). Indeed, a positive (negative) accrual indicates that there is a gain (loss) to be made in terms of social security wealth by staying on the labor force for one additional year. A negative accrual coefficient correctly predicts a decrease (increase) in the retirement probability following a positive (negative) accrual.

We find that age is highly correlated with our financial retirement incentive variables because they all capture the effect of eligibility to social security programs. Once we allow for non-linearity in age (second model), the coefficients of both the social security wealth and the accruals become smaller because they no longer capture the effect of eligibility to social security programs. As a matter of fact, once we include social security eligibility variables (third model), the accrual variable becomes insignificant.

Similarly to previous chapters, we find that both the age and the social security eligibility variables have a very strong effect on the retirement probability. In particular, in the second model, we find a significant effect of ages 58, 60 and 65, the standard eligibility age of conventional early retirement and the early and statutory eligibility ages of the oldage pension regime, respectively. ${ }^{279}$ Similarly, in the third model, we find a positive and strongly significant effect of our three social security eligibility variables.

ones. Thus, our estimation of pension benefits is less accurate than in chapter four. Our social security wealth and accrual variables might be further from their true value and does not enable us to find similar correlation between our financial retirement incentive variables and the retirement behavior. Second, our sample size is significantly lower in chapter four and this limits the variation of our social security wealth and accrual variables. Third, because we are working with survey data and incomplete earning histories, we had to discard observations of individuals for whom the wage and pension benefits variables were too low or too high (because of encoding error in the dataset or because of a miscalculation of their pension benefits). This limits even further the variation of our financial retirement incentives.

279 See chapter two for a list of the various eligibility ages for the old-age pension and the conventional early retirement. 
Corresponding to the findings of Maes (2008), we do not find a significant effect of our employment duration variables. ${ }^{280}$ This result hints at the fact that there is low duration dependence and once eligibility criteria are accounted for (through the age or eligibility variables), employment duration does not influence the retirement decision. Moreover, most of the effect of omitted variables that are correlated with time such as health are captured by the age regressor. In fact, we expect a positive bias of age on retirement since age and health are negatively correlated and health is plausibly negatively correlated with retirement.

We find that being a woman decreases the retirement probability by approximately 3 percent. This counter-intuitive result might be explained by our sampling methodology according to which we only keep women with relatively long careers and thus high taste for work, high motivation, low demand for leisure, etc. The level of education does not significantly influence the retirement decision, except for women with tertiary education who have a significantly higher probability of retirement compared to women with secondary education. It is possible that our financial incentive measures capture the effect of socio-economic status, which leads to an insignificant effect of education.

Unlike Coile (2004), Denaeghel et. al. (2014), Gustman and Steinmeier (2000), we find that spousal characteristics do not have a significant impact on the retirement decision. Interestingly, if we include individuals with lower pension benefits, spousal characteristics become significant. First, having a retired spouse has a positive and significant impact on the retirement probability and the effect is lower for women than for men, which corresponds to the findings of Blau (1998) and Gustman and Steinmeier

\footnotetext{
${ }^{280}$ We tried several functional form for employment duration : linear, cubic polynomial, log, dummies, group of 5 years. All were insignificant.
} 
(2000). Second, we find that having a younger spouse has a positive and significant impact on the retirement probability.

Working in the primary sector of activity leads to lower retirement probability compared to working in the secondary sector of activity. In contrast with our results in chapters 4 and 5, working part-time does not have an effect on the retirement probability. We tried interacting the part-time variable with gender but the effect remained non-significant. This result is potentially again caused by the sampling methodology that only allows for women with long careers and the way the part-time variable indicator is built (no information on work intensity for instance). The proportion of years worked as wage earner in total career does not have a significant impact on the retirement probability but following our sampling method, we only allow for a maximum of a 25 percent of nonwage-earner career. Finally, we find a small negative effect of the year of birth, indicating that younger workers tend to retire later, which corresponds to our results from chapter four.

We find no impact of being in a one-earner household on the retirement probability. It is important to note that part of the effect of being in a one-earner household is captured by the social security wealth variable because pension benefits are calculated using different replacement rates. Moreover, since we use a random effect model, we control for the effect of unobservables such as demand for leisure, taste for work, etc. It is plausible that since individuals in one-earner households are the only providers for the household, they tend to tune their retirement decisions to determinants linked to financial retirement incentives rather than personal or spousal characteristics. We tried controlling for an interaction between our financial retirement incentives variables and the one-earner household status but the coefficients were not significant, indicating that the financial incentive measures have the same effect between the different types of household. In fact, the predicted 
probability of retirement is higher for one-earner households, following the effect of social security wealth, which is higher for one-earner households.

Table 6.3: Discrete time logistic hazard duration model with random effects - regression results

\begin{tabular}{|c|c|c|c|}
\hline & $\begin{array}{l}\text { (i) Cubic age } \\
\text { polynomial }\end{array}$ & (ii) Age dummies & $\begin{array}{c}\text { (iii) Cubic age } \\
\text { polynomial and } \\
\text { eligibility variables }\end{array}$ \\
\hline $\begin{array}{l}\text { Social security wealth / } \\
100,000\end{array}$ & $\begin{array}{l}0.063^{* * *} \\
(0.023)\end{array}$ & $\begin{array}{l}0.041^{* *} \\
(0.019)\end{array}$ & $\begin{array}{c}0.081^{* * *} \\
(0.025)\end{array}$ \\
\hline Accrual / 100,000 & $\begin{array}{c}-0.267^{* * *} \\
(0.059)\end{array}$ & $\begin{array}{c}-0.153^{* * *} \\
(0.051)\end{array}$ & $\begin{array}{l}-0.071 \\
(0.046)\end{array}$ \\
\hline Last net wage (in $2017 €$ ) & $\begin{array}{l}-0.004^{*} \\
(0.002)\end{array}$ & $\begin{array}{l}-0.003^{*} \\
(0.002)\end{array}$ & $\begin{array}{l}-0.005^{* *} \\
(0.003)\end{array}$ \\
\hline Female & $\begin{array}{l}-0.037^{* *} \\
(0.018)\end{array}$ & $\begin{array}{l}-0.033^{* *} \\
(0.016)\end{array}$ & $\begin{array}{c}-0.070^{* * * *} \\
(0.024)\end{array}$ \\
\hline Primary school & $\begin{array}{c}0.027 \\
(0.028)\end{array}$ & $\begin{array}{c}0.020 \\
(0.024)\end{array}$ & $\begin{array}{c}0.046 \\
(0.036)\end{array}$ \\
\hline Tertiary education & $\begin{array}{c}0.015 \\
(0.018)\end{array}$ & $\begin{array}{c}0.013 \\
(0.016)\end{array}$ & $\begin{array}{c}0.028 \\
(0.024)\end{array}$ \\
\hline French speaker & $\begin{array}{l}-0.016 \\
(0.015)\end{array}$ & $\begin{array}{l}-0.013 \\
(0.013)\end{array}$ & $\begin{array}{l}-0.027 \\
(0.020)\end{array}$ \\
\hline Retired partner & $\begin{array}{c}0.007 \\
(0.019)\end{array}$ & $\begin{array}{c}0.004 \\
(0.017)\end{array}$ & $\begin{array}{c}0.005 \\
(0.021)\end{array}$ \\
\hline Older partner & $\begin{array}{c}0.003 \\
(0.019)\end{array}$ & $\begin{array}{c}0.002 \\
(0.017)\end{array}$ & $\begin{array}{c}0.008 \\
(0.026)\end{array}$ \\
\hline $\begin{array}{l}\text { Age difference with } \\
\text { partner }\end{array}$ & $\begin{array}{c}0.001 \\
(0.002)\end{array}$ & $\begin{array}{c}0.002 \\
(0.002)\end{array}$ & $\begin{array}{c}0.000 \\
(0.003)\end{array}$ \\
\hline One-earner household & $\begin{array}{l}-0.024 \\
(0.025)\end{array}$ & $\begin{array}{l}-0.006 \\
(0.022)\end{array}$ & $\begin{array}{l}-0.033 \\
(0.033)\end{array}$ \\
\hline Primary sector of activity & $\begin{array}{l}-0.077^{* *} \\
(0.037)\end{array}$ & $\begin{array}{l}-0.073^{* *} \\
(0.031)\end{array}$ & $\begin{array}{l}-0.091 \\
(0.057)\end{array}$ \\
\hline Tertiary sector of activity & $\begin{array}{l}-0.009 \\
(0.014)\end{array}$ & $\begin{array}{l}-0.007 \\
(0.013)\end{array}$ & $\begin{array}{l}-0.006 \\
(0.020)\end{array}$ \\
\hline Part-time & $\begin{array}{c}0.036 \\
(0.023)\end{array}$ & $\begin{array}{c}0.024 \\
(0.020)\end{array}$ & $\begin{array}{c}0.039 \\
(0.030)\end{array}$ \\
\hline Wage-earner proportion & $\begin{array}{c}0.007 \\
(0.014)\end{array}$ & $\begin{array}{c}0.008 \\
(0.013)\end{array}$ & $\begin{array}{c}0.001 \\
(0.020)\end{array}$ \\
\hline Birth year & $\begin{array}{l}-0.002^{*} \\
(0.001)\end{array}$ & $\begin{array}{l}-0.002 \\
(0.001)\end{array}$ & $\begin{array}{l}-0.002 \\
(0.002)\end{array}$ \\
\hline
\end{tabular}




$\begin{array}{lcc}\text { Age } & 10.111^{* * *} & 11.168^{* * *} \\ & (3.016) & (3.592) \\ \text { Age squared } & -0.170^{* * *} & -0.185^{* * *} \\ & (0.050) & (0.061) \\ \text { Age cubic } & 0.001^{* * *} & 0.001^{* * *} \\ & (0.000) & (0.000)\end{array}$

Age dummies

\begin{tabular}{|c|c|}
\hline 56 & $\begin{array}{l}-0.005 \\
(0.016)\end{array}$ \\
\hline 57 & $\begin{array}{l}-0.004 \\
(0.017)\end{array}$ \\
\hline 58 & $\begin{array}{l}0.046^{* *} \\
(0.021)\end{array}$ \\
\hline 59 & $\begin{array}{c}0.023 \\
(0.023)\end{array}$ \\
\hline 60 & $\begin{array}{c}0.240^{* * *} \\
(0.040)\end{array}$ \\
\hline 61 & $\begin{array}{c}0.055 \\
(0.040)\end{array}$ \\
\hline 62 & $\begin{array}{c}0.063 \\
(0.047)\end{array}$ \\
\hline 63 & $\begin{array}{c}0.057 \\
(0.052)\end{array}$ \\
\hline 64 & $\begin{array}{c}0.072 \\
(0.062)\end{array}$ \\
\hline 65 & $\begin{array}{c}0.706^{* * *} \\
(0.079)\end{array}$ \\
\hline
\end{tabular}

Eligibility EEA OAP

$0.035^{* *}$

Eligibility SEA OAP

(0.016)

$0.196^{* * *}$

$(0.033)$

$0.143^{* * *}$

Eligibility CER

$(0.023)$

Employment duration

0.035

0.019

0.003

$(0.025)$

$(0.023)$

(0.029)

Employment duration

$-0.000$

$-0.000$

0.000

(0.000)

(0.000)

(0.000)

Note: Marginal effects calculated at the mean. Robust standard errors are in parentheses. Interaction effects are not shown. ${ }^{* * *} \mathrm{p}<0.01,{ }^{* *} \mathrm{p}<0.05,{ }^{*} \mathrm{p}<0.1$. 


\subsubsection{SIMULATION OF A CHANGE IN THE GENEROSITY OF THE HOUSEHOLD RATE BENEFITS}

In this section, we simulate the effect of a change in the generosity of the household replacement rate on the retirement probability of individuals in one-earner households and on various poverty measures. First, we estimate the impact of a decrease of 15 percentage points of the household replacement rate to 60 percent, which is equivalent to abolishing the household replacement rate. Next, we look at the effect of increasing the household replacement rate in gradual steps of 5 percentage points starting from a replacement rate of 60 percent. This simulation allows us to get an insight on whether the household replacement rate leads to a work (dis)incentive ${ }^{281}$ and to put a figure on the role of the household replacement rate in pension adequacy.

\subsubsection{IMPACT ON THE RETIREMENT PROBABILITY}

In this section, we first analyse the change in retirement probability caused by the removal of the household replacement rate. Decreasing the household replacement rate to 60 percent has two effects: (i) on the social security wealth and (ii) on the accrual.

First, a decrease in the household replacement rate leads to a decrease in the social security wealth and thus a decrease in the retirement probability following the income effect observed in section 6.5.2. Indeed, the poorer the individual, the more likely he or she will stay in the labor force and the lower the retirement probability.

Second, a decrease in the household replacement rate also generates a change in the accrual. In fact, before the individual is eligible for old-age pension, a decrease in the

\footnotetext{
${ }^{281}$ See Blau (1997), Knapp (2014) and Michaud (2003) for an analysis of the impact of other programs targeted at the dependent spouse on retirement.
} 
generosity of the system leads to a lower work incentive and thus a higher retirement probability (i.e. the positive accrual decreases), because there is less to be gained by working for one additional year. However, after the individual gains access to the oldage pension regime, the reform creates a lower work disincentive and thus a lower retirement probability (i.e. the negative accrual increases) because there is less to be lost by working for one additional year. The total effect of the reform depends on whether the income or the substitution effect is higher and on whether the individual has access to old-age pension benefits.

We predict the retirement probability of workers in one-earner households using our second model with age dummies. In figure 6.10, we present the change in retirement probability in percentage points in the case of a removal of the household replacement rate. Under the 60 percent replacement rate scenario, the average predicted probability of retirement decreases by an average of 1.2 percent at all ages. The impact is larger at ages 60 and 64 with a decrease of the retirement probability of 2.1 percent at both ages. We note that the decrease in retirement probability is smaller at lower ages, because of the effect of the household replacement rate on the accrual. Indeed, the decrease in accrual caused by the removal of the household replacement rate leads to higher retirement probability before the individual has access to the old-age pension regime and offsets some of the effect of the social security wealth. Therefore, on average, the removal of the household replacement rate has a small negative effect on the retirement probability (i.e. a work incentive) and the effect is stronger once the individual has access to the old-age pension regime. 
Figure 6.10: Retirement hazard rates for workers in one-earner households - baseline (75\% replacement rate) and suppression of household replacement rate (60\% replacement rate)

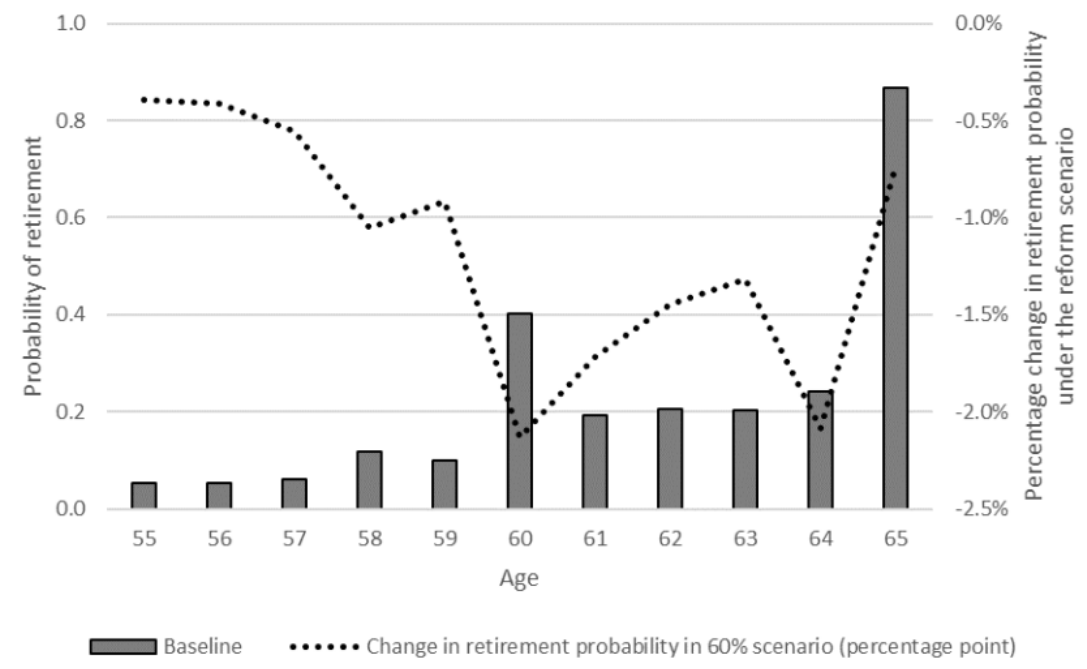

Source: Authors' own calculations using SHARE data

In figure 6.11, we look at the average change in retirement probability in percentage points by age groups (ages 55-59 and ages 60-65) for several scenarios of change in the generosity of the household replacement rate compared to the baseline scenario (75 percent replacement rate). Again, we observe that the effect is overall quite small. We find that a decrease in the household replacement rate leads to a decrease in the retirement probability and the effect is larger at older ages. An increase in the household replacement rate leads to an increase in the retirement probability and the effect is also larger at older ages.

In conclusion, we find that the removal of the household replacement rate would lead to a minor decrease in the retirement probability. In turn, an increase of the household replacement rate would lead to an increase in the retirement probability. Specifically, a change of 5 percentage points of the household replacement rate leads to a change in the retirement probability of approximately 0.24 percentage points below age 60 and 0.51 percentage points above age 59 and the effect is the largest at age 60. 
Figure 6.11: Change in retirement probability for individuals in one-earner households for different reform scenario of the household replacement rate

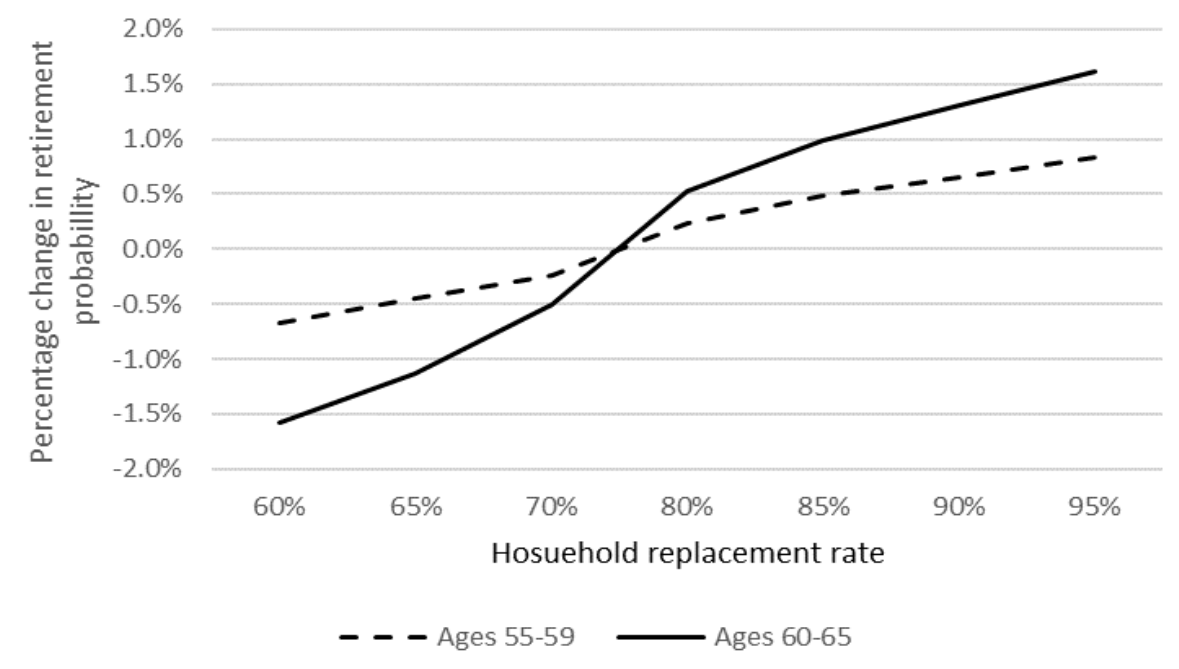

Source: Authors' own calculations using SHARE data

\subsubsection{IMPACT ON POVERTY MEASURES}

As highlighted in the introduction, the objectives of an adequate old-age pension system from a public policy perspective is poverty alleviation and income redistribution among the elderly (Barr and Diamond, 2006). The redistributive effect of the old-age pension program is embedded in the calculation of benefits through the minimum pension and the set of pensionable earnings minima and maxima, among other things. In addition, the household replacement rate serves as a redistribution mechanism between one-earner households and the rest of the population. Indeed, we test for the probability of being in a one-earner household using a probit model and we control for individuals and jobrelated characteristics. We find that being a male, having secondary education, working full-time and a lower total number of years of career all increase the probability of being in a one-earner household (see table 6.4). Most importantly, we find that being is a oneearner household is associated a lower average wage, which corresponds to the findings of Hindriks (2014) and the Pension Reform Committee (2014) that individuals in oneearner households are generally concentrated at the lower end of the income distribution. 
In this section, we present elderly poverty measures and we assess the impact of various reform scenarios of the household replacement rate on these same measures.

Table 6.4: Probit model of the probability of being in a one-earner household

\begin{tabular}{|c|c|}
\hline & Probit coefficients \\
\hline Estimated old-age pension & $0.003^{* * *}$ \\
\hline benefits & $(0.000)$ \\
\hline \multirow{2}{*}{ Last net wage } & $-0.000^{* * *}$ \\
\hline & $(0.000)$ \\
\hline \multirow{2}{*}{ Male } & $0.543^{* * *}$ \\
\hline & $(0.184)$ \\
\hline \multirow{2}{*}{ Primary education } & $-0.568^{* * *}$ \\
\hline & $(0.198)$ \\
\hline \multirow{2}{*}{ Tertiary education } & $-0.568^{* * *}$ \\
\hline & $(0.198)$ \\
\hline \multirow{2}{*}{ French speaker } & -0.169 \\
\hline & $(0.145)$ \\
\hline \multirow{2}{*}{ Retired partner } & $-0.596^{* * *}$ \\
\hline & $(0.156)$ \\
\hline \multirow{2}{*}{ Age difference (absolute value) } & 0.007 \\
\hline & $(0.026)$ \\
\hline \multirow{2}{*}{ Primary sector of activity } & -0.533 \\
\hline & $(0.373)$ \\
\hline \multirow{2}{*}{ Tertiary sector of activity } & -0.469 \\
\hline & $(0.382)$ \\
\hline \multirow{2}{*}{ Part-time work } & $0.567^{*}$ \\
\hline & $(0.295)$ \\
\hline \multirow{2}{*}{ Birth year } & $-0.104^{* * *}$ \\
\hline & $(0.012)$ \\
\hline \multirow{2}{*}{ Age } & -0.001 \\
\hline & $(0.012)$ \\
\hline \multirow{2}{*}{ Total years of career } & $-0.050^{* * *}$ \\
\hline & $(0.018)$ \\
\hline
\end{tabular}

Note: Table reports average marginal effects. Clustered standard errors are in parentheses. ${ }^{* * *} \mathrm{p}<0.01,{ }^{* *} \mathrm{p}<0.05,{ }^{*} \mathrm{p}<0.1$.

First, we estimate the old-age pension benefits entitlements of spouses using the same benefit calculation rules as for our sampled individuals and express the benefits into constant 2017 euros. Then, we sum the estimated old-age pension benefit of our sampled 
individuals and that of their spouses at the effective retirement age of the reference individual to obtain an indicator of the household income based on old-age pension benefits entitlements at retirement. ${ }^{282}$ Finally, in order to take into account differences in household size and economies of scales within the household, we transform the household old-age pension benefits entitlement into the equivalized household old-age pension income for a one-member household using the OECD modified equivalence scales $^{283}$.

We observe that pensioners in one-earner households have slightly higher average equivalized household old-age pension income (1185.10 euros) compared to individuals in two-earner households (1169.53 euros). Besides, the average equivalized household old-age pension income is lower for individuals in one-earner and two-earner households compared to singles (1494.89 euros). There are two reasons that can explain why individuals in one-earner households have higher average old-age pension benefits even though they have a lower average wage and there is only one prime-earner in the household: (i) they are granted the household replacement rate and (ii) they have characteristics that are associated with higher wages (for instance, male and less part-time work). Finally, we note that there is less variation in household old-age pension income for individuals in one-earner households than for other types of households (see figure $6.12)$.

\footnotetext{
${ }^{282}$ We restrict our sample to individuals who were retired at the time of the survey because we look at the old-age pension benefits they effectively receive at retirement and not their benefits entitlements. Therefore, we compute our poverty measures for a sample of 327 individuals.

283 We use the OECD modified equivalence scale that assigns a value of 1 to the household head and a value of 0.5 to each additional adult. (OECD, n.d.)
} 
Figure 6.12: Dispersion of equivalized old-age pension benefits by type of household

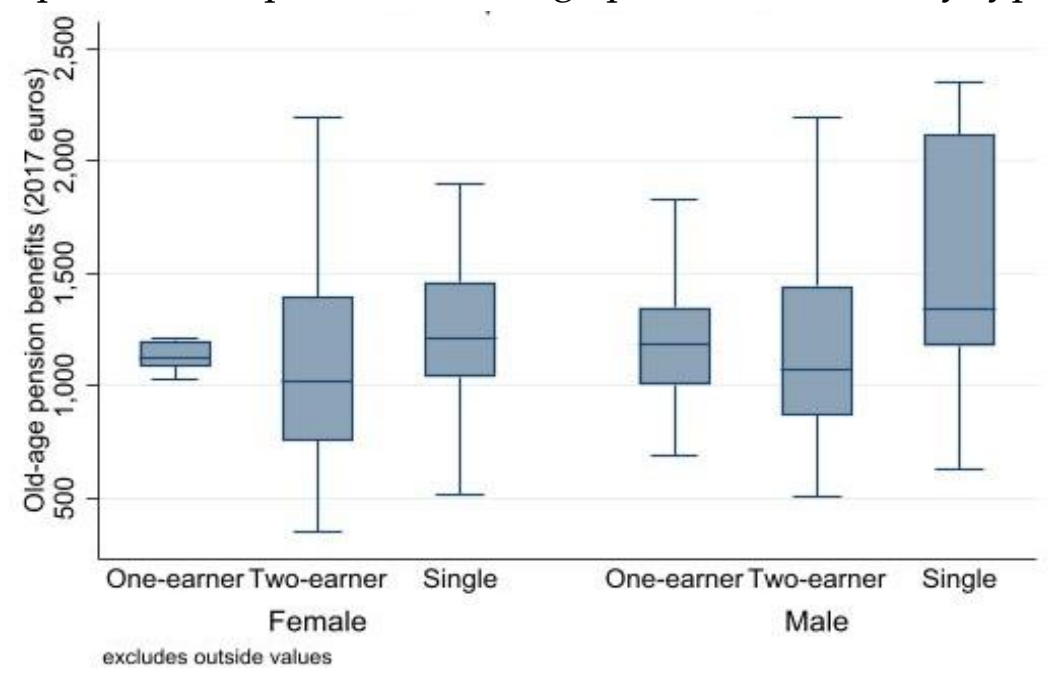

Source: Authors' own calculations using SHARE data

We measure the poverty of individuals ${ }^{284}$ at retirement using the equivalized household old-age pension income by means of the headcount ratio and the average poverty gap from the Foster, Greer and Thorbecke (1984) measures of poverty and a relative poverty line set at 50 percent of the median equivalized household old-age pension income in our sample. ${ }^{285}$ In our baseline scenario, we find a headcount poverty ratio of 5.77 percent for the sample and 2.75 percent for individuals in one-earner households.

Next, we look at the effect of increasing the household replacement rate in gradual steps of 5 percentage points starting from 60 percent. Figure 6.13 displays the average equivalized household old-age pension income (and its standard deviation) at retirement

\footnotetext{
${ }^{284}$ Because of data limitations, we calculate the poverty rate of individuals based on their old-age pension benefits entitlements only. For a complete assessment of old-age poverty rates, one should include house ownership and other types of revenues. 285 The Foster, Greer and Thorbecke (1984) poverty measure is written as $F G T(\alpha)=\frac{1}{n} \sum_{i=1}^{p}\left(\frac{z-X_{i}}{z}\right)^{\alpha}$. Where $\mathrm{X}$ is the equivalent household income for individual $\mathrm{i}$ and $\mathrm{z}$ is the poverty line. We use a relative poverty line set at $50 \%$ of the median individual old-age pension at retirement in our sample, which is equal to 648.67 euros (2017 euros). If $\alpha=0$, then the measure indicates the headcount ratio, or the proportion of the sample that lives in a poor household. If $\alpha=1$, then the measure indicates the intensity of poverty by adding up the relative difference between the household income and the poverty line.
} 
of individuals in one-earner households in each of these scenarios. For individuals in oneearner households, the removal of the household replacement rate would lead to a decrease of their average equivalized household old-age pension income from 1185.10 to 1010.34 euros, their headcount ratio would increase from 2.75 percent to 4.59 percent and their average poverty gap would increase from 0.79 percent to 1.33 percent of the poverty line. We observe that as the household replacement rate increases, so does the standard deviation, meaning that inequality between individuals in one-earner households increases.

Figure 6.13: Average equivalized old-age pension benefits by type of households under different reform scenarios of the household replacement rate

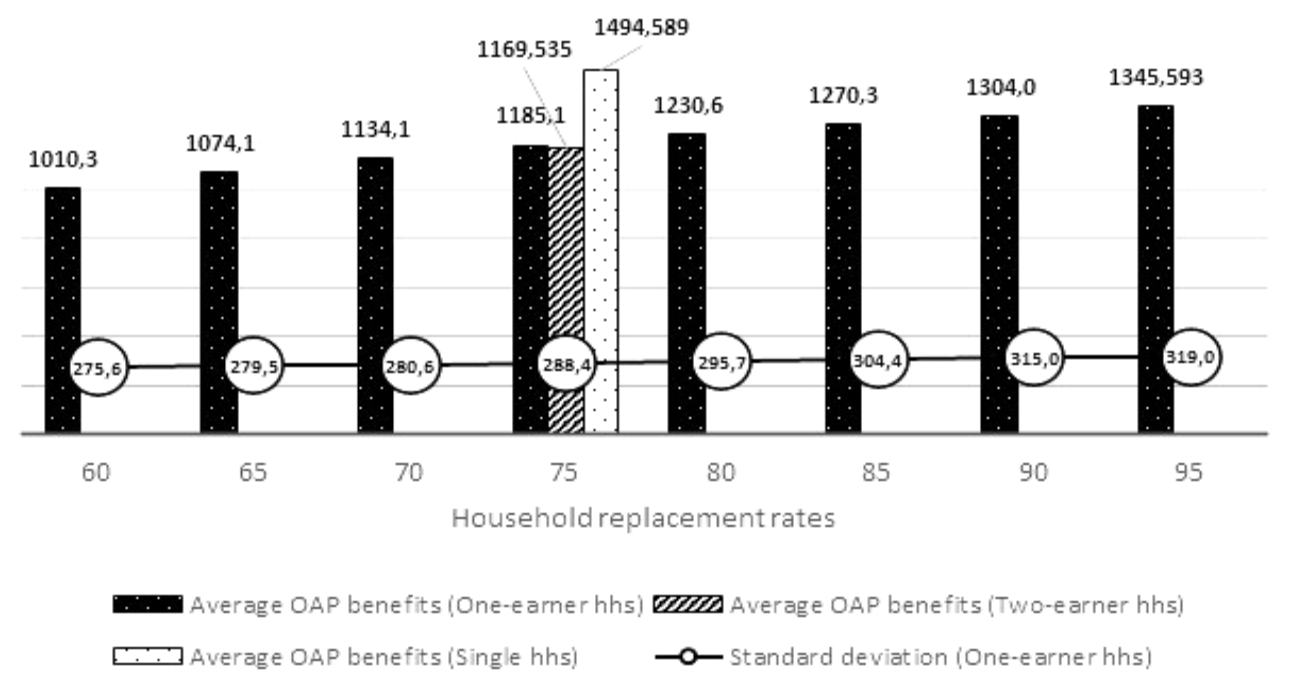

Source: Authors' own calculations using SHARE data

Figure 6.14 displays the headcount ratio and the average poverty gap for each household replacement rate reform scenario. We note that the decrease in both the poverty headcount ratio and average poverty gap slows down as the household replacement rate increases. 
Figure 6.14: Headcount ratio and average poverty gap of one-earner households under different household replacement rate reform scenarios
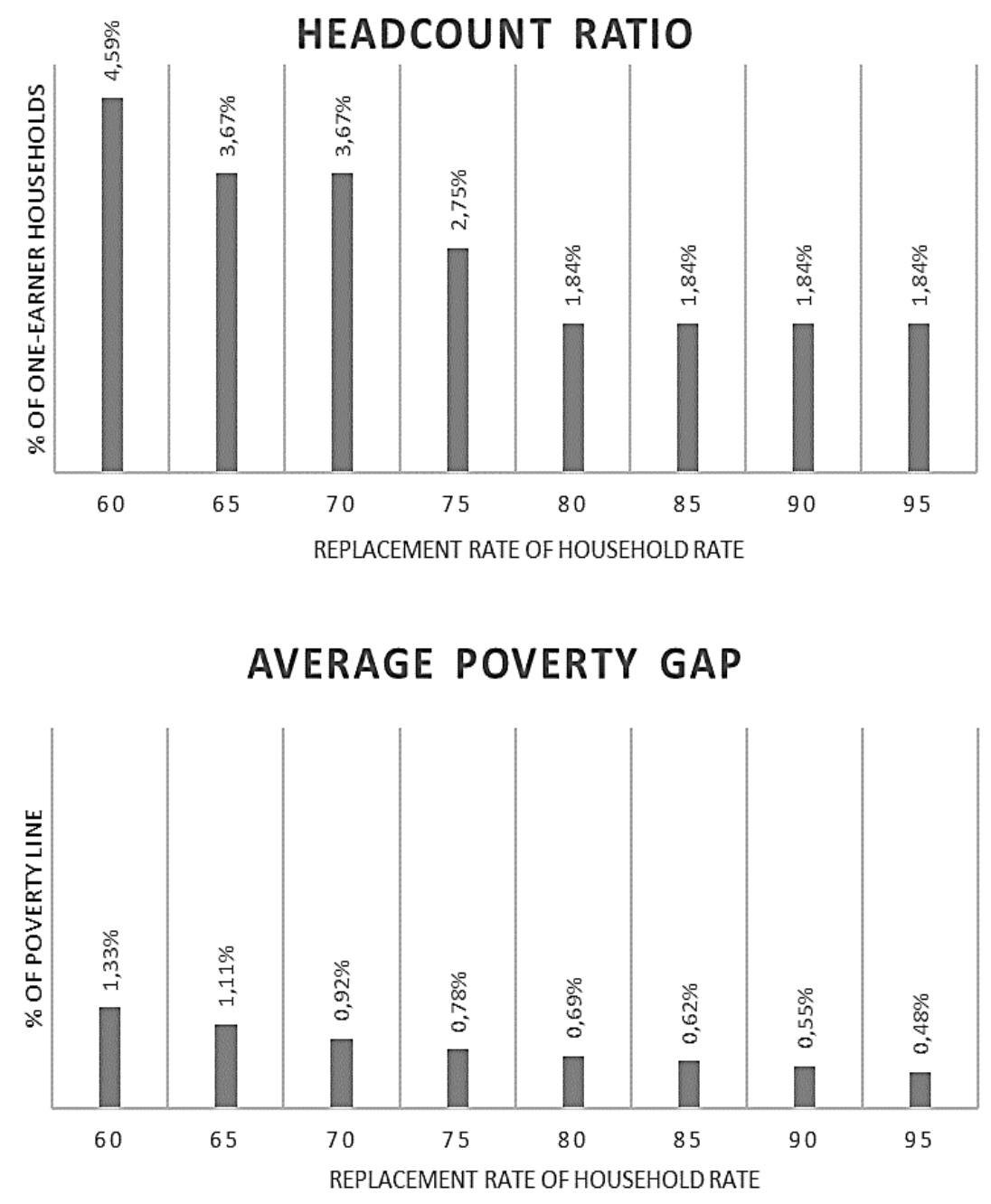

Source: Authors' own calculations using SHARE data 


\subsection{CONCLUSION}

In this chapter, we study the retirement incentives of workers in one-earner households. Pensioners in one-earner households are granted the more generous household replacement rate for the calculation of their old-age pension benefits, which takes the form of a 15 percentage point increase of the standard replacement rate. While one-earner households are generally concentrated at the lower end of the income distribution, their average pension benefits is generally above those of individuals in two-earner or single households because of the household replacement rate. However, we find that pensioners in one-earner households have the lowest average equivalized old-age pension benefits in our sample.

We use a discrete time logistic duration model to study the transitions from employment to retirement using a sample of older Belgian workers from the survey dataset SHARE. We construct financial retirement incentive measures (social security wealth and accruals) and find that they have a significant effect on retirement. Firstly, we find that the higher the social security wealth, the higher the retirement probability. This result thus correctly predicts the income effect of social security benefits according to which an individual with higher social security wealth will leave the labor force earlier. Since pensioners in oneearner households have higher average social security wealth thanks to the household replacement rate, the income effect of social security benefits is larger for them than for other types of households. Secondly, we find that the lower the accrual, the higher the retirement probability. Thus correctly predicting the substitution effect of social security benefits according to which the higher the returns of an additional year of work, the lower the retirement probability. After controlling for financial retirement incentives, we find that the effect of being a worker in a one-earner household on the retirement probability is insignificant. Moreover, we find that the effect of the financial retirement incentives on 
the retirement probability is not significantly different according to the type of household the worker lives in.

We estimate the effect of a change in the household replacement rate on the retirement probability of individuals in one-earner households. We start by comparing our baseline scenario $(75 \%$ replacement rate) to a scenario in which the household replacement rate is set to 60 percent, which is equivalent to suppressing it. We find that such a reform would create a work incentive because of the ensuing decrease in social security wealth. The impact of the removal of the household replacement rate on the accruals is slightly more complex. In fact, before the individual is eligible for old-age pension, a decrease in the generosity of the system leads to a lower work incentive (i.e. the positive accrual decreases), because there is less to be gained by working for one additional year. However, after the individual gains access to the old-age pension regime, the reform creates a lower work disincentive (i.e. the negative accrual increases) because there is less to be lost by working for one additional year. We find that the total effect of removing the program would have a negative effect on the retirement probability, which would be more important at older ages. Similarly, we find that an increase in the household replacement rate would lead to a limited increase in the retirement probability of individuals in one-earner households. Unsurprisingly, increases in the generosity of the household rate benefits lead to substantial decreases in the poverty measures among the elderly. However, this effect becomes smaller as the replacement rate increases

In view of these results, we advocate in favour of the recommendation of the Belgian Pension Reform Committee to remove the household replacement rate except for minimum pensions. Since households with asymmetrical working arrangements are often at the lowest part of the income distribution, the substantial effect of the household 
replacement rate on poverty measures is motivating. Moreover, we have found only low working incentives imbedded in the program.

Despite the continued need for income redistribution among the elderly to avoid the persistence of income inequality into old age, such redistribution need not be targeted at financially dependent spouses, especially given that we are witnessing an increase of the modernized male breadwinner model and a rise in divorces and durable legal cohabitation arrangements. Indeed, pensionable earning minima, minimum pension benefits and the pension benefits calculation accounting for periods spent on replacement income actually serve the income redistribution goal. 


\section{REFERENCES}

Ageing Study Committee. (2020). Rapport annuel 2020. Bruxelles: Conseil supérieur des finances.

Albanese, A., Cockx, B., and Thuy, Y. (2020). Working time reductions at the end of the career: Do they prolong the time spent in employment?, Journal of Empirical Economics, $59,99-141$.

Andriopoulou, E., and Tsakloglou, P. (2011). The determinants of Poverty Transitins in Europe and the Role of Duration Dependence. IZA Discussion paper series.

Antolin, P., and Scarpetta, S. (1998). Microeconometric analysis of the retirement decision: (OECD) Economics Department Working Papers 204.

Aranki, T., and Macchiarelli, C. (2013). Employment Duration and Shifts into Retirement in the EU. LEQS Paper No. 58/2013.

Baker, M. (2002). The retirement behavior of married couples evidence from the spouse's allowance. Journal of Human Resources, 37, 1-34.

Barr, N., and Diamond, P. (2006). The economics of Pensions. Oxford Review of Economic Policy, 22(1), 15-39.

Berghman, J., Curvers, G., Palmans, S., and Peeters, H. (2007). Cartographie des retraités belges. Partie 1: la protection offerte par la pension légale. Sécurité Sociale. Working Paper, 6.

Blau, F. (1998). Trends in the Well-Being of American Women, 1970-1995. Journal of Economic Literature, 36(1), 112-165.

Börsch-Supan, A., and Coile, C. (2020). Social Security Programs and Retirement around the World: Reforms and Retirement Incentives. Chicago: University of Chicago Press.

Ciccia, R., and Kotowska, I. (2014). After the Male Breadwinner Model? Childcare Services and the Division of Labor in European Countries. Social Politics, 21(1), 50-79. 
Coile, C. (2004). Retirement Incentives and Couple's Retirement Decisions. Topics in Economic Analysis and Policy, 4(1).

Coile, C. (2015). Economic determinants of workers' retirement decisions. Journal of Economic Surveys, 29(4), 830-853.

Coile, C., and Gruber, J. (2001). Social Security Incentives for Retirement. In D. Wise (Ed.), Themes in the Economics of Aging. Chicago: University of Chicago Press.

Dekkers, G., Buslei, H., Cozzolino, M., Desmet, R., Geyer, J., Hofmann, D., . . Verschueren, F. (2009). What are the consequences of the AWG-projections for the adequacy of social security pensions? An application of the dynamic micro simulation model MIDAS for Belgium, Italy and Germany. Bruxelles: Federal Planning Bureau.

Denaeghel, M. B. (n.d.). Spousal influences on the retirement decisions of single-earner and two-earner couples. Advances in Life Course Research, 16(3), 112-123.

Diamond, P., and Gruber, J. (1999). Social Security and Retirement in the United States. In D. Wise, Social Security and Retirement Around the World (pp. 437-473). National Bureau of Economic Research.

Diamond, P., and Hausman, J. (1984). Individual retirement and savings behavior. Journal of Public Economics, 23(1-2), 81-114.

Eurostat. (2020). Duration of working life - Annual data. Retrieved from Eurostat: https://ec.europa.eu/eurostat/databrowser/view/lfsi_dwl_a/default/table?lang=en

Euwals, R., Van Vuuren, D., and Wolthoff, R. (2006). Early retirement behavior in the Netherlands. Tinbergen Institute Discussion paper 021/3.

Foster, J., Greer, J., and Thorbecke, E. (1984). A class of decomposable poverty indices. Econometrica, 52, 761-766.

Grech, A. (2012). Evaluating the possible impact of pension reforms on future living standards in Europe. CASE paper 161.

Grech, A. (2013, April). How best to measure pension adequacy. Munich Personal RePEc Archive (461226). 
Gruber, J., and Wise, D. (1999). Social Security and Retirement around the World. Chicago: University of Chicago Press.

Gruber, J., and Wise, D. (2004). Social Security and Retirement around the World: Microestimation. Chicago: University of Chicago Press.

Gustman, A., and Steinmeier, T. (2000). Retirement in Dual-Career Families: A Structural Model. Journal of Labor Economics, 18(3), 503-545.

Hindriks, J. (2015). Quel avenir pour nos pensions? Les grands défis de la réforme des pensions. Bruxelles: Pierre de Boeck.

Höhn, C., Avramov, D., and Kotowska, I. (20008). People, Population Change and Policies. European Studies of Population, 16(2).

Holzmann, R., and Hinz, R. (2005). Old Age Income Support in the 21st Century: An international perspective on pension systems and. Washington D.C.: The World Bank.

Jenkins, S. (1995). Easy Estimation Methods for Discrete-time duration Models. Oxford Bulletin of Economics and Statistics, 57(1).

Jenkins, S. (2000). Modelling household income dynamics. Journal of Population Economics, 13, pp. 529-567.

Jousten, A., and Tarantchenko, E. (2014). New evidence on the social security incentives as drivers of retirement behavior. SSRN.

Knapp, D. (2014). The effect of social security auxiliary spouse and survivor's benefits on the household retirement decision. 16th Joint annual Meeting of the Retirement Research Consortium.

Lindeboom, M. (1998). Microeconometric analysis of the retirement decision: the. (OECD, ed.) Economics Department Papers 207.

Maes, M. (2008). Financial and redistributive impact of reforming the old-age pension system in Belgium. Discussion Papers (ECON - Département des Sciences Economiques) 2008040. 
Meghir, C., and Whitehouse, E. (1997). Labor market transitions and retirement of men in the Netherlands. Journal of econometrics, 79, 327-354.

Michaud, P. (2003). Joint labor supply dynamics of older couples. IZA Discussion Paper(832).

Michaud, P., and Vermeulen, F. (2004). Labor supply of elderly couples: a collective. CentER.

Minguez, A. M. (n.d.). The persistence of male breadwinner model in southern European countries on a compared perspective: Familism, employment and family policies. Marie Curie Annals, 4.

National Pension Office. (1992-2019). Annual reports 1992-2019. Bruxelles: Service Fédéral des Pensions.

OECD. (n.d.). Adjusting household incomes: equivalence scales - OECD. Retrieved from OECD: http://www.oecd.org/economy/growth/OECD-Note-EquivalenceScales.pdf

Pension Reform Committee 2020-2040. (2014). Un contrat social performant et fiable. Bruxelles: SPF Sécurité Sociale.

Pension Reform Committee 2020-2040. (2014). Un contrat social performant et fiable. Annexe 3.4: La modernisation de la dimension familiale. Bruxelles: SPF Sécurité Sociale.

Schills, T. (2008). Early Retirement in Germany, the Netherlands, and the United Kingdom: A Longitudinal Analysis of Individual Factors and Institutional Regimes. European Sociological Review, 24(3), 315-329.

Spataro, L. (2002). New tools in micromodelling retirement decisions: overview and. CERP Working Paper 28.

Statbel. (2020). Les indicateurs de pauvreté en Belgique en 2017 (EU-SILC). Retrieved from Statbel: https://statbel.fgov.be/fr/nouvelles/les-indicateurs-de-pauvrete-enbelgique-en-2017-eusilc\#: :text=Les\%20principales\%20conclusions\%20sont\%20les,mois\%20pour\%20une\%20 personne $\% 20 \mathrm{isol} \% \mathrm{C} 3 \% \mathrm{~A} 9 \mathrm{e}$. 
Statistics Belgium. (2015). Tendances sur le marché du travail belge (1983-2013). SPF Economie.

Stock, J., and Wise, D. (1990a). Pensions, the option value of work, and retirement. Econometrica, 58, 1151-1180.

Stock, J., and Wise, D. (1990b). The pension inducement to retire: an option value analysis. In D. Wise (Ed.), Issues in the economics of Aging. Chicago: University of Chicago Press.

Wolthoff, R., Euwas, R., and Vuuren, D. (2006). Early Retirement Behavior in the Netherlands: Evidence from a Policy Reform. SSRN Electronic Journal. 


\section{APPENDIX}

\section{A.1 HOUSEHOLD RATE BENEFITS AROUND EUROPE}

\begin{tabular}{|c|c|c|c|}
\hline Country & $\begin{array}{l}\text { Form of household rate } \\
\text { benefits }\end{array}$ & $\begin{array}{l}\text { Percentage } \\
\text { pensioners }\end{array}$ & Discontinuation \\
\hline $\begin{array}{l}\text { United- } \\
\text { Kingdom }\end{array}$ & $\begin{array}{l}\text { The dependent's } \\
\text { supplement is paid for a } \\
\text { dependent adult if the } \\
\text { earnings from work are } \\
\text { below a specified amount. }\end{array}$ & $\begin{array}{l}16.000 \text { individuals or } \\
0.2 \% \text { of } 12.980 .800 \\
\text { pensioners in } 2106 \\
76.700 \text { individuals in } \\
2006 \text { or } 0.7 \% \text { of total } \\
11.734 .100 \text { number of } \\
\text { pensioners }{ }^{286}\end{array}$ & $\begin{array}{l}\text { New claims are } \\
\text { no longer } \\
\text { possible. The } \\
\text { payment of the } \\
\text { supplement will } \\
\text { cease in April } \\
2020\end{array}$ \\
\hline France & $\begin{array}{l}\text { Dependent's supplement } \\
\text { for a spouse older than age } \\
65 \text { (or } 60 \text { in case of } \\
\text { disability), who does not } \\
\text { receive any social security } \\
\text { benefits or personal } \\
\text { resources that exceed } 833 € \\
\text { monthly }\end{array}$ & $\begin{array}{l}105.540 \text { individuals in } \\
2015 \text { or } 0.8 \% \text { of } \\
13.041 .056 \quad \text { total } \\
\text { number of pensioners } \\
287\end{array}$ & $\begin{array}{l}\text { No new claims } \\
\text { since January } \\
2011\end{array}$ \\
\hline $\begin{array}{l}\text { The } \\
\text { Netherlands }\end{array}$ & $\begin{array}{l}\text { Supplementary allowance } \\
\text { for younger partner: the } \\
\text { pensioner must be aged } 65 \\
\text { or older and the partner } \\
\text { younger than age } 65 \text { with } \\
\text { income from employment } \\
\text { less than } € 1,324.46 \text { a month } \\
\text { (or income from benefits } \\
\text { less than } € 734.41 \text { a month). } \\
\text { The allowance is paid until }\end{array}$ & $\begin{array}{l}162.454 \text { at the end of } \\
2015 \text { or } 4.8 \% \text { of } \\
3.371 .258 \text { total } \\
\text { pensioners in } 2015^{288} \\
313.374 \text { at the end of } \\
2012 \text {, or } 10.4 \% \text { of } \\
\begin{array}{l}3.016 .955 \text { total } \\
\text { pensioners }\end{array}\end{array}$ & $\begin{array}{l}\text { No new claims } \\
\text { since January } \\
2015\end{array}$ \\
\hline
\end{tabular}

${ }^{286}$ Retrieved from http://tabulation-

tool.dwp.gov.uk/5pc/sp/ccdepind/ccbentyp/a_stock_r_ccdepind_c_ccbentyp_sep06.html

${ }^{287}$ Retrieved from https://www.lassuranceretraite.fr/portail-info/files/live/sites/pub-

bootstrap/files/pdf/rapports-documents-reference/Abrege-15-site-en-ligne.pdf

${ }^{288}$ Retrieved from

http://www.svbkennisplatform.nl/FbContent.ashx/pub_1002/downloads/v1606151328/KB\%202015\%204e

$\% 20$ kwartaal.pdf (p.28, p.30) and

http://www.svbkennisplatform.nl/kennisbank/zoeken.kerncijfers/a1447_Kerncijfers-SV-en-Niet-SV 


\begin{tabular}{|c|c|c|}
\hline & $\begin{array}{l}\text { the younger partner is } 65 \text {. } \\
\text { An earnings test on the } \\
\text { household income exists. }\end{array}$ & \\
\hline Belgium & $\begin{array}{l}\text { A supplement of } 15 \% \text { of the } \\
\text { average past } 45 \text { years of } \\
\text { career is granted if the } \\
\text { spouse receives a work } \\
\text { income or social security } \\
\text { benefits below a certain } \\
\text { threshold. }\end{array}$ & $\begin{array}{l}307.261 \text { individuals in } \\
2016 \quad(305,626 \text { men } \\
\text { and } 1636 \text { women })^{289} \text {, } \\
\text { or } 15.2 \% \text { of total of } \\
2.015 .338 \text { pensioners } \\
349.766 \text { individuals in } \\
2006 \text { or } 20 \% \text { of } \\
1.747 .111 \text { total } \\
\text { number of pensioners }\end{array}$ \\
\hline Norway & $\begin{array}{l}\text { Income tested dependent's } \\
\text { supplement for a dependent } \\
\text { spouse who does not } \\
\text { receive an personal OAP } \\
\text { benefits }\end{array}$ & $\begin{array}{l}3063 \text { individuals in } \\
2009 \text { or } 0.5 \% \text { of } \\
647388 \text { pensioners } \\
2028 \text { or } 0.2 \% \text { of } 889043 \\
\text { total pensioners }{ }^{290} \text { in } \\
2015\end{array}$ \\
\hline Ireland & $\begin{array}{l}\text { Dependent's supplement is } \\
\text { paid for a dependent spouse } \\
\text { with an income below a } \\
\text { certain threshold, varies } \\
\text { with the dependent } \\
\text { spouse's age. }\end{array}$ & $\begin{array}{l}3162 \text { individuals or } \\
0.6 \% \text { of } 95.570 \text { total } \\
\text { number of recipients } \\
\text { of non-contributory } \\
\text { state pension in } 2014 \\
291 \\
72.193 \text { individuals or } \\
13.8 \% \text { of } 522.244 \text { total } \\
\text { number of pensioners } \\
\text { in } 2011\end{array}$ \\
\hline Portugal & $\begin{array}{l}\text { Dependent's } \\
\text { supplement }\end{array}$ & \\
\hline Cyprus & $\begin{array}{l}\text { Dependent's spouse } \\
\text { supplement increases the } \\
\text { basic pension to } 80 \% \text { of the } \\
\text { average past earnings }\end{array}$ & \\
\hline
\end{tabular}

${ }^{289}$ Retrieved from

http://www.onprvp.fgov.be/RVPONPPublications/FR/Statistics/Annual2016/FR_Statistique_2016.pdf

${ }^{290}$ Retrieved from https://www.nav.no/no/NAV+og+samfunn/Statistikk/Pensjon++statistikk/Alderspensjon

${ }^{291}$ Retrieved from https://www.welfare.ie/en/downloads/Social-Stats-AR-2014-SectionB.pdf and https://www.welfare.ie/en/Pages/Annual-SWS-Statistical-Information-Report-2014.aspx 


\begin{tabular}{|c|c|c|}
\hline Isle of Man & & \\
\hline \multicolumn{3}{|c|}{ Changes in minimum or maximum pension } \\
\hline Italy & $\begin{array}{l}\text { Increase of minimum } \\
\text { monthly OAP benefits if the } \\
\text { annual income of the } \\
\text { household is below a certain } \\
\text { threshold }\end{array}$ & \\
\hline Spain & $\begin{array}{l}\text { Increase of minimum and } \\
\text { maximum monthly OAP } \\
\text { benefits if the annual } \\
\text { income of the household is } \\
\text { below a certain threshold }\end{array}$ & \\
\hline Sweden & $\begin{array}{l}\text { Higher social pension if in a } \\
\text { couple. }\end{array}$ & \\
\hline Austria & $\begin{array}{l}\text { Higher social pension if in a } \\
\text { couple. }\end{array}$ & \\
\hline Greece & $\begin{array}{l}\text { Increase of minimum } \\
\text { monthly OAP benefits if the } \\
\text { annual income of the } \\
\text { household is below a certain } \\
\text { threshold }\end{array}$ & \\
\hline
\end{tabular}


6.A.2 EVOLUTION OF MONTHLY GROSS SALARY BY AGE (AVERAGE 1999 2017)

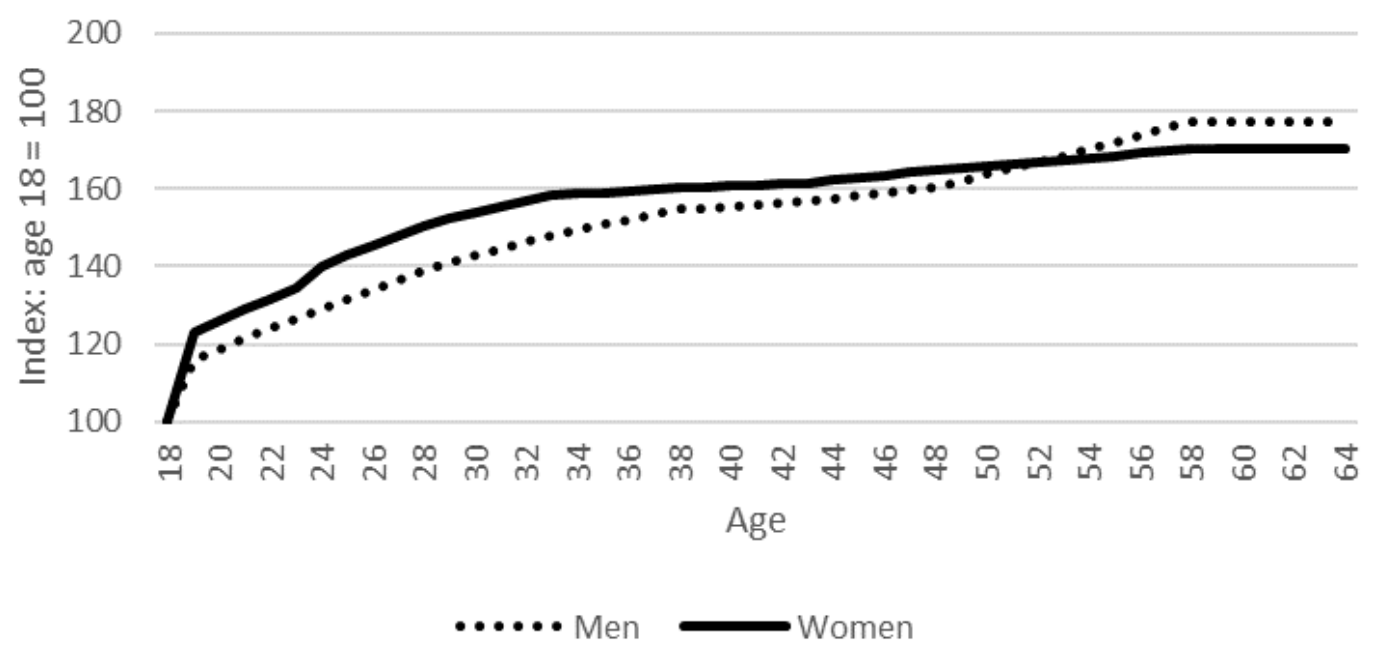

Source: Statbel (2019) 


\title{
CONCLUSION
}

\author{
Anne-Lore Fraikin
}


Rapid population ageing and the influx of baby boomers arriving at the age of retirement is generating a considerable threat for the financial sustainability of Belgium's pay-asyou-go old-age pension system. Indeed, the steep increase in the old-age dependency ratio is predicted to be translated into a rise in gross public pension expenditures of 6.6 percentage points of GDP between 2016 and 2070. In addition to these demographic trends, Belgium counts with relatively low elderly labor force participation rates and a low average effective retirement age.

The necessary reforms to safeguard the level of old-age pension benefits in payment are essentially composed of three measures: increased contributions and tax financing, lowered benefits and increases in the effective retirement age. In Belgium, the government has implemented various reforms with the objective of delaying the effective retirement age. On the one hand, access to early labor force exit routes outside of the old-age pension system has been tightened. On the other hand, efforts have been made to reduce the incentives of early claim of old-age pension benefits. Although the elderly employment rates have been increasing since the mid-1990's, this trend is not enough to absorb the negative effect of population ageing and efforts to improve the financial sustainability of the country's old-age pension system should be actively pursued.

Next to pressing sustainability matters, it is also important that pension reforms take account of pension adequacy measures, or the ability of the system to provide a replacement income that prevents individuals from old-age poverty and allows for consumption smoothing at older ages. While elderly poverty rates have been decreasing over the last few years and are now comparable to those of the general population, they are relatively higher than most neighbouring countries.

The conjunction of the increase in elderly labor force participation and the wave of reforms that tightened the coverage and generosity of the social security system has 
spawned a vast literature on the link between the retirement decision and financial retirement incentives stemming from the social security system. The objective of the thesis is to update past studies and to shed further light on the mechanisms that determine the retirement behavior of older workers. We use detailed social security eligibility and benefit calculation rules and their reforms from the nineteen eighties until today to compute measures of financial retirement incentives and we study their impact on retirement using Belgium as a case study.

\subsection{KEY FINDINGS AND POLICY IMPLICATIONS}

In this section, we summarize key findings of each chapter and we link them to our policy recommendations.

Chapter two - Historical evolution of the Belgian social security landscape for older workers

The second chapter presents a thorough review of the Belgian social security landscape relevant for the study of retirement. We demonstrate that the system has become increasingly complex and sometimes fragmented, because of a lack of long-term planning and reforms piling on top of one another with little coherence, which brings about a particularly challenging situation for the analysis of its effects on the retirement decision.

Because of such complexity, it is essential that the effect of sustainability reforms is assessed in terms of both sustainability and adequacy and that any potential intertwined effect between programs or reforms is considered. For instance, the 1997 reform that increased the statutory eligibility age of women from 60 to 65 had the expected effect of delaying their retirement age, which improved the sustainability of the pension system as a whole. However, the period of benefits receipt of certain replacement income programs (e.g. conventional early retirement) also increased as beneficiaries of these programs are automatically rolled over to the old-age pension regime at the statutory eligibility age. On 
top of this direct fiscal cost, this reform also created differed fiscal liabilities and led to an increase of social security wealth for women receiving these benefits as periods spent receiving a replacement income count towards the pension history, be it at the rate of the last wage or a lower lump-sum amount. Since access these benefits generally requires a minimum number of years of career, one can plausibly assume that the reform also generated a rise in social security wealth inequality between women with short or parttime careers and other women. However, the same reform might have improved social security wealth inequality between healthy and unhealthy women by extending the period of disability benefits that can be included in the calculation of old-age pension benefits. In light of such a complex effect of reforms, there is a need for cautious ex-ante and ex-post analysis and for policy makers to have a good grasp of the overall current social security system and its evolution over time.

Chapter three - Social security incentives in Belgium: an analysis of four decades of change

The third chapter of the thesis examines the evolution of financial retirement incentives generated by the Belgian social security system from the eighties until the present day. More specifically, we construct a microsimulation model aimed at assessing the incentives to retire for different subgroups of the population (differentiated by their sex, level of earnings, marital status) for each retirement age between 55 and 65, retirement years from 1980 to 2016 and for each of the four traditional labor force exit pathways. We show that the social security reforms that occurred in Belgium have had a significant impact on the financial retirement incentives of older workers. More specifically, we observe that Belgian workers face an implicit tax on additional work above age 60, and sometimes as early as age 58. Therefore, we find that the wide array of (early) labor force exit pathways does create a situation in which certain older workers would be better off (in terms of social security wealth) by leaving the labor force early. 
Chapter four - Trends in social security incentives in Belgium

The fourth chapter of the thesis explores the empirical link between financial retirement incentive measures and the retirement behavior of older Belgian workers. We use an administrative panel dataset for years 2004 to 2010 to construct various financial retirement incentive variables and test their correlation with the retirement decision of workers at the micro level. We find that there is a positive and significant income effect and a negative and significant substitution effect of financial retirement incentives on the retirement probability, which is slightly higher for women than for men. Namely, higher pension wealth leads to earlier retirement and higher marginal returns to work lead to later retirement, respectively. Moreover, we simulate the impact of various reform scenario on the retirement probability and we find that reforms that tightened the eligibility conditions of (early) labor force exit pathways had a relatively large effect on the financial retirement incentives compared to reforms of benefit calculation rules (e.g. pension bonus).

In view of these results, it appears that reforms tightening the eligibility rules of social security programs are a powerful policy tool and are central in ensuring the long-term financial sustainability of the pension system. Furthermore, from a policy perspective, the abolition of the pension bonus can be considered as consistent as its removal effectively leads to later retirement through a decreased income effect. Finally, we find slightly different results between men and women and thus advocate for a separate analysis of the impact of social security reforms on the retirement between men and women.

Chapter five - Work, labor force exit and benefit claiming patterns in Belgium

The complexity of the Belgian social security system sometimes leads workers to optimally or not - disjoin labor force exit from (early) benefit claiming decisions by opting for the time credit program for instance. The fifth chapter of the thesis documents the 
dissociation of the retirement decision into a labor market exit and a benefit claiming decision and explores its impact from a research and a public policy point of view. Such a dissociation leads us to consider workers and retired individuals differently according to their benefit claiming status. We use a detailed panel administrative dataset to study the retirement and benefit claiming trends of older Belgian workers. Once we decompose the recent increase in elderly employment rates, we note that an important part of it is attributable to a rise in part-time work (mostly for women) and in the group of workers who are claiming social security benefits (mostly time-credit, disability and unemployment benefits). Moreover, we find that the proportion of workers who are claiming benefits has been increasing faster than other types of workers over the last decade. Finally and most importantly, we find that workers who are claimers (resp. individuals in part-time working arrangements) have a higher probability to exit the labor force early compared to workers who are not claimers (resp. workers in full-time working arrangements).

In conclusion, when assessing the financial sustainability of the pension system, the decomposition of employment and retirement trends according to the benefit claiming status proves to be of prime relevance.

First, social security reforms can potentially influence both decisions differently - possibly disjoining them - and the impact evaluation of a reform should include its effect on both decisions separately. As a matter of fact, we study the determinants of both decisions independently and find that they are influenced by distinct factors.

Second, the option of combining (part-time) work with social security benefits claiming influences labor market decisions at older ages. In fact, some authors argue that this combination might encourage some workers to stay on the labor force longer. We show 
that workers who are claiming social security benefits (e.g. time credit benefits) tend to retire earlier compared to workers who are not claiming any benefits. Moreover, while some of these programs might indeed lead workers to retire later, they might also induce some workers who would have stayed employed full-time to retire partly. Therefore, our results indicate that allowing for older workers to gradually reduce their working hours (through a program such as the time credit) does not lead to the desired policy effect, that of delaying retirement. Consequently, the effect of such work arrangements or programs and the direction of causality should be examined in more detail before access to gradual retirement programs is made more or less tight.

Third, distinguishing labor force exit from benefit claiming decisions and identifying both short-and long-term fiscal costs of benefit claims are two issues of prime policy relevance in Belgium. The country has a highly complex landscape of labor force exit and benefit claiming options and the ubiquitous nature of partial or full labor force exit options and the absence of penalty for early claimers of old-age pension benefits render the short and the long-run fiscal stakes particularly high. Consequently, in pension sustainability prediction analysis, workers who are claiming social security benefits should be considered separately from other workers and their current and differed fiscal costs should be analyzed carefully.

Chapter six - The old-age pension household replacement rate for pensioners in one-earner households

Pensioners in one-earner households are granted the more generous household replacement rate for the calculation of their old-age pension benefits, which takes the form of a 15-percentage point increase of the standard replacement rate. In the sixth chapter, we examine the retirement incentives of workers in one-earner households, and we look 
at the impact of the household replacement rate of the old-age pension system on pension sustainability and adequacy measures.

We use a discrete time logistic duration model to study the transitions from employment to retirement using a sample of older Belgian workers from the survey dataset SHARE. Specifically, we test whether the program generates higher (lower) work (dis)incentives through its impact on our financial retirement incentive measures. We find a positive effect for our social security wealth measure, correctly predicting that higher social security wealth leads to earlier retirement, and a negative effect of the accrual, correctly predicting that higher marginal return to work leads to later retirement. Overall, we find that the household replacement rate leads to slightly earlier retirement because the income effect generated by its impact on social security wealth is larger than the substitution effect generated by its impact on accruals. Moreover, we find a substantial effect of the household replacement rate on poverty measures of individuals in one-earner households, which slows down as the generosity of the household replacement rate increases.

Since households with asymmetrical working arrangements are often at the lowest part of the income distribution, we advocate in favour of the recommendation of the Belgian Pension Reform Committee to remove the household replacement rate, with the exception of minimum pensions. Indeed, despite the need for income redistribution among the elderly to avoid the persistence of inequality into old age, such redistribution need not be targeted at financially dependent spouses, especially given the rise of the modernized male breadwinner model and an increasing number of divorces or durable legal cohabitation arrangements. Indeed, pensionable earning minima, minimum pension benefits and the pension benefits calculation accounting for periods spent on replacement 
income effectively serve the income redistribution goal and do not favour a certain type of household over another.

\subsection{LIMITATIONS AND DIRECTIONS FOR FUTURE RESEARCH}

In this section, we detail some of the limitations of our analysis (e.g. caused by the data structure) and we give some insights into some of the research topics that appear stimulating and valuable for future analysis in view of our results.

Because of data limitations, we focus on the analysis of the retirement determinants of wage earners and leave aside the self-employed and civil servant working regimes. Indeed, there is only a limited amount of available information for self-employed workers (e.g. the income information is missing) in the administrative dataset retrieved from the Crossroad Bank of Social Security that we use in chapters 3, 4 and 5. In addition to data limitations, information on the evolution of the social security rules for self-employed and civil servants is scarce. Furthermore, there exists a wide array of sub-systems in the civil servant regime, each with specific eligibility and benefit calculation rules, which makes it a particularly challenging regime to study. We suppose that the retirement decision of self-employed workers (resp. civil servants) and wage earners are influenced by different factors, as the later have access to a substantially less (resp. more) generous social security system and are thus plausibly less (resp. more) influenced by financial retirement incentives. Further analysis on the way financial retirement incentives influence the retirement determinants of self-employed and civil servant workers would be an extremely valuable input into the current literature. However, this analysis should imperatively be done separately for the different regimes because of the vast disparity that exists in terms of social security eligibility and benefit calculation rules. 
In the first chapter, we summarize the evolution of benefit calculation and eligibility rules of the main early labor force exit pathways for older Belgian workers. Research papers on how these reforms have interacted with one another and their impact in terms of both pension sustainability and adequacy is infrequent. Future research on the intertwined effects of reforms and between early labor force exit pathway programs would be desirable and provide an insightful tool in the public policy debate. Such additional analysis would inevitably serve to reinforce our conclusion that the Belgian social security system has become increasingly convoluted, which complexifies the study of retirement and of the sustainability of the pension system.

In the fourth chapter, we find that the impact of our financial retirement incentives differ slightly between men and women, with our SSW and ITAX measures having a higher significant impact on the retirement probability of older women compared to older men. Additionally, we find that the impact of our social security eligibility age indicators is slightly higher for older men than for older women. Further analysis on the root causes of such differences and the way social security reforms influence the retirement behavior of men and women separately would be a valuable upgrade for the literature.

As far as the computation of financial retirement incentives is concerned, we advocate for the inclusion of as many social security calculation rules as possible in future research papers. Indeed, some calculation or eligibility rules that appear trivial may well have an important impact on financial retirement incentives (e.g. taxation) while other programs have only a limited impact on such incentives (e.g. pension bonus). Besides, there still exists gender differences in social security eligibility rules and it is imperative that such specificities are included as parameters for the model to account for the actual incentives faced by workers. It is difficult to predict how such an upgrading would have influenced our financial retirement incentives measures as the inclusion of any additional parameter could in principle influence our measure both ways. 
For data limitation reasons, we were not able to generate a "true" eligibility indicator for some of the programs. For instance, we could not capture true eligibility to the conventional early retirement system, as there are too many special regimes with specific eligibility rules (for workers in companies in distress, in arduous jobs, etc.). This undoubtedly affects our computation of financial incentive measures and the analysis of the effect of our eligibility indicator on retirement. Indeed, the work disincentives associated with some early labor force exit programs are a lot higher compared to the disincentives created by the old-age pension system. We can thus assume that our financial incentive measures are biased downward because we were not able to include some of the incentives created by these programs.

Moreover, the lack of detailed information on eligibility to some of the early labor force exit programs prohibits us from computing an improved aggregation mechanism of financial retirement incentive, in which the aggregated measure would only be influenced by the incentives originating from the social security programs the worker has access to.

Owing to data limitations, we did not include a health indicator in our regression analyses. We expect the health status to play an active role in the determination of retirement. Therefore, we assume that our regressions suffer from an omitted variable bias. While there is a wide array of research that looks at the impact of health on retirement, our analysis would have benefitted from the inclusion of health as a covariate. In general, further research on the intertwined effect between the financial retirement incentives and health would be worthwhile.

Further research on the impact of part-time work and the combination of work and benefit claiming on the retirement behavior would be profitable. Indeed, while these work arrangements might entice some workers to stay on the labor force longer, they might also induce some workers to opt out of a full-time employment without claiming benefits 
to retire partially through these programs. Overall, our results from chapter five seem to indicate that allowing for the combination of work and benefit claiming at older ages might not lead to the expected effect of encouraging older workers to stay on the labor force longer. Besides, following the important proportion of older women working parttime, a differentiated and detailed analysis of the effect of such programs on retirement between men and women would be extremely valuable. Finally, further research is needed to establish the direction of causality between part-time work at older ages and retirement. From a methodological point of view, our analysis could have benefited from a nested structure approach that would have included the many different retirement pathways that are available for older Belgian workers. Leaving aside methodological and data impracticalities of this approach, this model upgrade could have had substantial impact on our results as we would have been able to better represent the actual choice set of the worker.

Finally, a large-scale analysis of the retirement behavior of individuals in one-earner households would be beneficial. Indeed, our sample size was rather limited owing to the fact that we were working with survey data. Likewise, the analysis of the impact of the household replacement rates on the work incentives of the financially dependent spouse (typically the woman) in a one-earner household is also a relevant topic to examine and remains understudied to this date. 


\section{SUMMARY}

Rapid population ageing and the influx of baby boomers arriving at the age of retirement is generating a considerable threat for the financial sustainability of Belgium's pay-asyou-go old-age pension system. In addition to these demographic trends, Belgium must cope with relatively low elderly employment rates and one of the earliest average effective retirement ages in the OECD. Concurrently to a trend observed in many developed countries, the country has witnessed a major transformation of its labor market environment over the last decades. The employment rate of elderly Belgian men exhibits a u-shaped curve - with a decline in the 1980's and 1990's and then an upward trend starting in the 2000's. The employment rate of elderly Belgian women is characterized by a long-term upward trend, reflecting a structural increase in the labor force participation of women.

While these increases in employment rates show potential for improving the financial sustainability of the pension system, these tendencies are in no way sufficient to absorb the overwhelming burden of population ageing on public old age spending. Confronted with predictable demographic trends, the Belgian government has implemented various social security reforms since the 1990's to restrict the impact of population ageing on the financial sustainability of the country's pension system. On the one hand, access to early labor force exit routes outside of the old-age pension system has been tightened. On the other hand, efforts have been made to reduce the incentives of early old-age pension benefits claiming.

The objective of the present thesis is to explore the impact of the social security system's provisions as a driver of the retirement behavior of older workers, using Belgium as a case study. We use detailed social security eligibility and benefit calculation rules and their 
reforms from the nineteen eighties until today to compute measures of financial retirement incentives and we study their impact on retirement using Belgium as a case study.

The second chapter of the thesis contains a thorough review of the Belgian social security landscape relevant for the study of retirement. We illustrate the complexity and sometimes incoherency of the Belgian social security system, which generates an enabling environment for intertwined effects between programs and reforms. The third chapter presents a microsimulation model aimed at assessing the evolution of financial retirement incentives stemming from the social security system from the 1980s to the present day for different subgroups of the population. We update previous studies by using recent data and we calculate the financial incentives to exit employment for various typical workers, differentiated by age, year, income level, marital status and sex - integrating changes both in benefits and in the tax system. The aim of the fourth chapter is to examine the empirical link between the financial retirement incentives created by the social security system and the retirement decisions of older Belgian workers. We update previous studies in terms of period coverage by using a more recent panel administrative dataset and we provide additional simulations of reforms. The fifth chapter documents the dissociation of the retirement decision into a labor market exit and a benefit claiming decision. Increasingly, individuals face more complex choice sets, that sometimes lead them to - optimally or not - disjoin these two decisions. We consider four different sub-statuses of individuals, based on work and benefit claiming statuses and we examine the impact of this decomposition from a public policy and a retirement analysis point of views. The objective of the sixth chapter is to examine the retirement behavior of individuals in oneearner households, i.e. households composed of one earner and one partner who is financially dependent. These individuals are automatically granted a more generous replacement rate in the calculation of their old-age pension benefits, the so-called 
household replacement rate. We provide an ex-ante impact evaluation of the removal of the household replacement rate on both pension sustainability and pension adequacy measures. 


\section{ABOUT THE AUTHOR}

Anne-Lore was born in Liege, Belgium on the $7^{\text {th }}$ of April 1989. She holds a Bachelor's degree in Management Science and Economics from Liege University in Belgium received in 2012, a Master's degree in Management Science with a specialization in Strategic Intelligence and Marketing from Liege University in Belgium received in 2014 and a Master's degree in Public Policy and Human Development with a specialization in Social Policy Design and Financing from Maastricht Graduate School of Governance (MGSoG)/United-Nations University (UNU) in the Netherlands received in 2015. During her studies, Anne-Lore completed a university exchange program in the Universidad de Santiago de Chile in Chile, an internship at the Walloon Export Office in Beijing and an internship at the International Labour Organization country office of the Philippines, where she was involved in the Assessment Based National Dialogue (ABND) that assessed the coverage gaps of the country's social protection system.

Anne-Lore Fraikin joined the Ph.d. program in Innovation, Economics and Governance for Development at UNU-MERIT and the Ph.D. program in Economics and Management at Liège University (HEC-Ulg) within the Tax Institute in 2016. She received a full scholarship from the Belgian Fund for Scientific Research (FNRS FRESH) and a full scholarship from the United-Nations University in 2016. Anne-Lore specializes in labour economics, population economics, public economics and microeconometrics. As part of her thesis, she co-authored two chapters in the National Bureau of Economic Research book series "International Social Security Around the World". She worked under the supervision of Pr. Dr. Alain Jousten and Pr. Dr. Alessio Brown.

During her Ph.d., Anne-Lore realized a research stay at the Institute of Public Policy based at the Paris School of Economics, where she examined the retirement behavior of older 
French workers. She was also a temporary lecturer for the course Public Finance and Political Economy in Belgium and Europe for MSc. students in Economics at the University of Liege and a tutor for the course of Quantitative Methods in Social Protection Policy Design taught in cooperation with the International Labour Organizations for MSc. students in Public Policy and Human Development at Maastricht Graduate School of Governance (MGSoG)/United-Nations University (UNU). 


\section{UNU-MERIT/MGSOG DISSERTATION SERIES}

2021

\section{Bart Kleine Deters}

A Quantitative approach to the right to education: Concept, Measurement, and Effects

UNU-MERIT/MGSoG Dissertation

Series № 260

\section{Anne-Lore Fraikin}

The effect of financial retirement incentives

originating from the social security system on the retirement behavior of older Belgian workers

UNU-MERIT/MGSoG Dissertation

Series № 261

\section{Eliana Rubiano-Matulevich}

Essays on Conflict-Induced Displacement and Gender in Colombia

UNU-MERIT/MGSoG Dissertation

Series № 259

\section{Njagi Purity Muthoni}

Financial Risk, Vulnerability and Equity of Access to Healthcare Services in Kenya UNU-MERIT/MGSoG Dissertation Series № 258

\section{María José Espinosa-Romero}

The Shadow of Hierarchy in Marine Fisheries Governance UNU-MERIT/MGSoG Dissertation Series № 257

\section{Lorena Rivera León}

Unveiling the Determinants of Scientific Productivity in Middle-Income Countries:

An Economics of Science Perspective UNU-MERIT/MGSoG Dissertation Series № 256

\section{Racky Balde}

Essays on Informal versus formal Economy Choices

UNU-MERIT/MGSoG Dissertation

Series № 255

\section{Caio Torres Mazzi}

Learning, Capabilities and Governance in Global Value Chains

UNU-MERIT/MGSoG Dissertation

Series № 254 


\section{Giulia Rossello}

Social Transformations and Labour Market

Entry

An Investigation into University Systems in

Emerging Economies

UNU-MERIT/MGSoG Dissertation

Series № 253

2020

\section{Rose Camille Vincent}

Essays in Public Economics

Multi-Layer Tax Structure and Implications

UNU-MERIT/MGSoG Dissertation

Series № 252

\section{Emmanuel Mensah}

Structural change in developing countries:

patterns, causes, and consequences

UNU-MERIT/MGSoG Dissertation

Series № 251

\section{Ornsaran Manuamorn}

Governance of International Adaptation

Finance for Local Climate Change

Adaptation: An Analysis of Adaptation

Fund Projects

UNU-MERIT/MGSoG Dissertation

Series № 250

\section{Gillian McFarland}

Doing policy in Further Education

An exploration of the enactment of the

GCSE resits policy in Further Education

colleges in England

UNU-MERIT/MGSoG Dissertation

Series № 249

\section{Omar Rodriguez Torres}

Essays on Entrepreneurship in Colombia

UNU-MERIT/MGSoG Dissertation

Series № 248

\section{Elaine Lebon-McGregor}

International Organizations and Global

Migration Governance

UNU-MERIT/MGSoG Dissertation

Series № 247

\section{Janyl Moldalieva}

Playing the 'Game' of Transparency and Accountability in Kyrgyzstan's Resource

Governance

UNU-MERIT/MGSoG Dissertation

Series № 246

\section{Kaleab Kebede Haile}

Essays on Rural Household Decision-

Making under Climate Risk

UNU-MERIT/MGSoG Dissertation

Series № 245

\section{Nora Jasmin Ragab}

Diaspora Mobilisation in a Conflict Setting

UNU-MERIT/MGSoG Dissertation

Series № 244

\section{Mary Kaltenberg}

From Micro to Macro: Essays on

Technological Change and Income

Divergence

UNU-MERIT/MGSoG Dissertation

Series № 243 


\section{Ayla E. Bonfiglio}

Student migrant, refugee or both?

Exploring Refugee Agency and Mobility through Tertiary Education in Kenya, South

Africa and Uganda

UNU-MERIT/MGSoG Dissertation

Series № 242

\section{Danilo Sartorello Spinola}

Cycles, Economic Structures and External Constraints.

A Structuralist study on the causes of economic volatility in Latin America UNU-MERIT/MGSoG Dissertation Series № 241

2019

\section{Jemal Adem}

Livelihood Vulnerability to Shocks, Behaviour and Investment in Education:

Essays in Behavioural Development Economics

UNU-MERIT/MGSoG Dissertation

Series № 240

\section{Davina Osei}

Corrupt or corrupted networks?

An empirical enquiry

UNU-MERIT/MGSoG Dissertation

Series № 239

\section{Patima Chongcharoentanawat}

Beyond Static Inequality

Public policies and economic mobility in

Thailand

UNU-MERIT/MGSoG Dissertation

Series № 238

\section{Charlotte Guillard}

Rethinking economic growth

and structural change.

The role of boundaries and linkages between industries

UNU-MERIT/MGSoG Dissertation

Series № 237

\section{Nicolas Echarti}

Employment Effects or Vocational

Rehabilitation in Germany:

A quantitative analysis

UNU-MERIT/MGSoG Dissertation

Series № 236

\section{Shellie E. Solomon}

Neighborhoods Matter:

Crime, collective efficacy and foreclosures in Miami

UNU-MERIT/MGSoG Dissertation

Series № 235

\section{Michał Kazimierczak}

Regional Innovation and Entrepreneurship Patents, trade marks, entry and entrants' growth in European manufacturing industries

UNU-MERIT/MGSoG Dissertation Series № 234

\section{Fernanda Soares}

The Influence of Within School and Across Schools' Collaborative Practices on Student Learning and Teaching Outcomes in West Africa

UNU-MERIT/MGSoG Dissertation Series № 233 


\section{Mira Bierbaum}

New Mindsets to Innovate Activation

UNU-MERIT/MGSoG Dissertation

Series № 232

\section{Norman Dytianquin}

Technology in the Asian Miracle and Crisis Debates: Applications of and Insights from the Field of Influence Approach to InputOutput Analysis

UNU-MERIT/MGSoG Dissertation

Series № 231

\section{Nga Le}

The implications of health insurance for the labour market and patient satisfaction with medical care in Vietnam

UNU-MERIT/MGSoG Dissertation

Series № 230

\section{Jinhyuck Park}

Intellectual Property right protection and cross-border RED investments by multinational enterprises

UNU-MERIT/MGSoG Dissertation

Series № 229

\section{Richard de Groot}

Show me the Money:

Essays on the Impact of Cash Transfers on Child Nutrition and the Role of Intra-

Household Dynamics

UNU-MERIT/MGSoG Dissertation

Series № 228

\section{Catie Lott}

Diamonds are a Women's Best Friend

Broadening Measures of Women's Access to

Formal Political Decision-Making

UNU-MERIT/MGSoG Dissertation

Series № 227

\section{Ana Cristina Calderon Ramirez}

Public Management Reforms

Three stories about public procurement agencification in Latin America UNU-MERIT/MGSoG Dissertation

Series № 226

\section{Camilo Nicanor Carrillo Purin}

Teachers' in-service training and student achievement:

The effect of in-service training of Peruvian teachers on student achievement

UNU-MERIT/MGSoG Dissertation

Series № 225

\section{Hugo Confraria}

Developing scientific capacity in the Global South

UNU-MERIT/MGSoG Dissertation

Series № 224

\section{Alison Cathles}

Educational Pathways and Skills:

Past, Present, and Future

UNU-MERIT/MGSoG Dissertation

Series № 223

\section{Ibrahima Sory Kaba}

Aggregate Fluctuations and Development:

Essays on Macroeconomic Volatility and

Economic Growth

UNU-MERIT/MGSoG Dissertation

Series № 222

\section{Charlotte Keijser}

Firm Participation, Learning and

Innovation in Heterogenous Value Chains of IT-enabled Services

UNU-MERIT/MGSoG Dissertation

Series № 221 


\section{Salih Çevikarslan}

Innovation Strategies and Their

Implications for Technological Change and

Market Outcomes:

An Evolutionary Multi-Agent Based

Modelling Approach

UNU-MERIT/MGSoG Dissertation

Series № 220

\section{Wondimagegn Mesfin Tesfaye}

Essays on the Impacts of Climate-Smart

Agricultural Innovations on Household

Welfare

UNU-MERIT/MGSoG Dissertation

Series № 219

\section{Tatevik Poghosyan}

How Board Networks Affect Firm

Performance and Innovation Incentives in

Transition Economies: The Case of Armenia

UNU-MERIT/MGSoG Dissertation

Series № 218

\section{Arip Muttaqien}

Essays on Inequality and Polarization:

Empirical Studies in Developing Asia

UNU-MERIT/MGSoG Dissertation

Series № 217

\section{8}

\section{Katrin Marchand}

Essays on Forced Migration and Labour

Market Participation in Developing

Countries

UNU-MERIT/MGSoG Dissertation

Series № 216

\section{Ortrun Merkle}

The Myth of Gender Neutral Power:

Corruption and Gender Norms

UNU-MERIT/MGSoG Dissertation

Series № 215

\section{Biljana Meshkovska}

Life after Trafficking:

(re)integration processes of women that have been trafficked for the purpose of sexual exploitation in Europe

UNU-MERIT/MGSoG Dissertation

Series № 214

\section{Vincenzo Vinci}

The Relevance of Institutions and People's

Preferences for Social Protection

UNU-MERIT/MGSoG Dissertation

Series № 213

\section{Silke Heuser}

The Effectiveness of Environmental Policies on Reducing Deforestation in the Brazilian Amazon

UNU-MERIT/MGSoG Dissertation

Series № 212

\section{Jennifer Waidler}

Social Assistance and Remittances and Their Role in the Fight Against Poverty

UNU-MERIT/MGSoG Dissertation

Series № 211

\section{Choolwe Muzyamba}

The role of community mobilization in the promotion of maternal health of women living with HIV in Zambia

UNU-MERIT/MGSoG Dissertation

Series № 210 
Juan Carlos A. Castillo Sánchez

Assessing the Role of the Export Sector in

Mexican Economic Development,1965-2014

UNU-MERIT/MGSoG Dissertation

Series № 209

\section{Tareq Abuelhaj}

Food Security Policy Impact Analysis: The

Econometrics of Cash and Food Assistance

Cost Effectiveness

UNU-MERIT/MGSoG Dissertation

Series № 208

\section{Marta Férnandez de Arroyabe Arranz}

Essays on MEAS and Innovation

UNU-MERIT/MGSoG Dissertation

Series № 207

\section{Clotilde Mahé}

Essays on Migration and Occupational

Choice

UNU-MERIT/MGSoG Dissertation

Series № 206

\section{Simone Sasso}

Talent on the move. Essays on Human

Capital, Graduate Mobility and Economic

Development

UNU-MERIT/MGSoG Dissertation

Series № 205

\section{Khaled Walid Rajab}

Strategic Planning under Fragility

UNU-MERIT/MGSoG Dissertation

Series № 204

\section{Mutinta Hambayi Nseluke}

A Tall Order: Improving Child Linear

Growth

UNU-MERIT/MGSoG Dissertation

Series № 203

\section{Elvis Korku Avenyo}

Innovations and Firm Performance

in sub-Saharan Africa: Empirical Analyses

UNU-MERIT/MGSoG Dissertation

Series № 202

\section{Ni Zhen}

Employment Dynamics, Firm Performance

and Innovation Persistence in the Context of

Differentiated Innovation Types: Evidence

from Luxembourg

UNU-MERIT/MGSoG Dissertation

Series № 201

\section{Caroline Wehner}

Too Scared to Achieve: The Relation

Between Neuroticism, Conscientiousness

and Socioeconomic Outcomes

UNU-MERIT/MGSoG Dissertation

Series № 200

\section{Stefania Innocenti}

On Institutional Persistence

UNU-MERIT/MGSoG Dissertation

Series № 199

\section{Hassen Abda Wako}

Economic Globalization, Institutions and

Development: Essays on Aid, Foreign Direct

Investment and Trade

UNU-MERIT/MGSoG Dissertation

Series № 198

2017

\section{Hans-Erik Edsand}

Winds of Change

UNU-MERIT/MGSoG Dissertation

Series № 197 


\section{Ana Patricia Silva Vara}

Redressing the Gender Gap

UNU-MERIT/MGSoG Dissertation

Series № 196

\section{Andrés Iván Mideros Mora}

Essays on the Economic Effects of Noncontributory Social Protection

UNU-MERIT/MGSoG Dissertation

Series № 195

\section{Tobias Broich}

New Actors in the Global Economy

UNU-MERIT/MGSoG Dissertation

Series № 194

\section{Bernard Nikaj}

From No-government to E-government

UNU-MERIT/MGSoG Dissertation

Series № 193

\author{
Ali Safarnejad \\ Prioritizing the HIV Response \\ UNU-MERIT/MGSoG Dissertation \\ Series № 192 \\ Clovis Freire \\ Diversification and Structural Economic \\ Dynamics \\ UNU-MERIT/MGSoG Dissertation \\ Series № 191

\section{Michael Verba} \\ Innovation and Knowledge Dynamics: \\ Essays on the Knowledge Economy \\ UNU-MERIT/MGSoG Dissertation \\ Series № 190
}

\section{Pui Hang Wong}

The Hearts and Minds in Conflict and Peace: The Economics of Counterinsurgency and the Psychology of Reconstruction UNU-MERIT/MGSoG Dissertation Series № 189

\section{Brenda Yamba}

Schooling Despite All Odds: Evidence from Lesotho on Female Child Carers who Stayed in School

UNU-MERIT/MGSoG Dissertation

Series № 188

\section{Sheng Zhong}

Moving towards An Energy Efficient

Future: Essays on Energy Efficiency, Technology and Development

UNU-MERIT/MGSoG Dissertation

Series № 187

\section{Julieta Marotta}

Access to Justice and Legal Empowerment of Victims of Domestic Violence through Legal Organizations in the City of Buenos Aires: A Qualitative Empirical Legal Study UNU-MERIT/MGSoG Dissertation Series, № 186

\section{Andrea Franco-Correa}

On the Measurement of Multidimensional Poverty as a Policy Tool: Empirical Applications to Chile, Colombia, Ecuador and Peru UNU-MERIT/MGSoG Dissertation Series, № 185 
2016

\section{Yesuf Awel}

Insurance for Growth: Empirical Essays on

Insurance Demand and Impacts in Africa

UNU-MERIT Dissertation Series, № 108

\section{Tigist Mekonnen Melesse}

Grow More Food using Fewer Resources:

Agricultural Technology Adoption and

Innovation Practices for Inclusive and

Sustainable Development

UNU-MERIT Dissertation Series, № 107

\section{Eleni Yitbarek}

Getting Ahead or left Behind? Essays on

Poverty Dynamics and Social Mobility in

Africa

UNU-MERIT Dissertation Series, № 106

\section{Thuy Dieu Nguyen}

Firm-Level Theory and Evidence of

Corruption

UNU-MERIT Dissertation Series, № 105

\section{Raquel Tsukada Lehman}

Essays on Household Production with

Labor-Saving Technology

UNU-MERIT Dissertation Series, № 104

\section{Eva Barteková}

Multi-Problem Challenges for a Renewable

Future: Empirical Studies on Competitive

Disadvantages from Electricity Price

Differentials and Mineral Supply Risk in an

Open Economy

UNU-MERIT Dissertation Series, № 103

\section{Jocelyn Olivari}

Entrepreneurial Traits and Innovation:

Evidence from Chile

UNU-MERIT Dissertation Series, № 102

\section{Muhammad Shafique}

Essays on the role of knowledge, $R \mathcal{E} D$, and

Technology-based Firms in the Evolution of

Socio-techno-economic System

UNU-MERIT Dissertation Series, № 101

\section{Serdar Türkeli}

Governance of Innovation Policy: Empirical Studies on Applied Political Economy by Multi-Methods Analysis

UNU-MERIT Dissertation Series, № 100

\section{Ayokunu Adedokun}

Pathways to Sustainable Peace building in

Divided Societies: Lessons and Experiences

from Mozambique

MGSoG Dissertation Series, № 75

\section{Luiz Rothier Bautzer}

Organizing Concurrent Engineering through ICT Platforms

Blueprinting Product Lifecycle Management Platforms across Disciplinary Agencies

MGSoG Dissertation Series, № 74

\section{Natalia Popova}

Migration in the Periphery of the European Union:

Determinants of Successful and Sustainable Labour Market Integration of Return Migrants in Albania, Egypt, Moldova and Tunisia

MGSoG Dissertations Series, № 73 


\section{Richard A. Martina}

Uncertainty and Resource Constraint in the

Small Island Developing States:

Essays in Entrepreneurial Cognition

MGSoG Dissertations Series, № 72

\section{Cécile Cherrier}

The Expansion of Basic Social Protection in

Low-income Countries:

An Analysis of Foreign Aid Actors' Role in

the Emergence of Social Transfers in Sub-

Saharan Africa

MGSoG Dissertations series, № 71

\section{Paul Caldron}

The Tacit Bargain in Short-Term Medical

Missions: Why U.S. physicians go and what it costs

MGSoG Dissertation Series, № 70

\section{Mahmut Kobal}

Customs \& Excellence: A Comparative

Approach on Administrative and Regulatory

Compliance Perspectives of the EU-Turkey

Customs Union

MGSoG Dissertation Series, № 69

\section{Craig Loschmann}

Essays on Conflict-related Migration and

Development in the Case of Afghanistan

MGSoG Dissertations Series, № 68

\section{Andrea Milan}

Rural Livelihoods, Location and Vulnerable

Environments: Approaches to Migration in

Mountain areas of Latin America

MGSoG Dissertation Series, № 67

\section{Farida Lada}

On Guarding the Welfare of Clinical Trial

Subjects While Promoting Novel Drug

Innovation

A Game Theoretical Approach

MGSoG Dissertation Series, № 66

\section{5}

\section{Hibret Belete Maemir}

Dissecting Aggregate Productivity:

International Integration and Growth with

Heterogeneous Firms

UNU-MERIT Dissertation Series, № 96

\section{Giorgio Triulzi}

Looking for the Right Path: Technology

Dynamics, Inventive Strategies and

Catching-up in the Semiconductor Industry

UNU-MERIT Dissertation Series, № 95

\section{Abdul Baseer Qazi}

Knowledge flows and networks in the ICT sector: The case of Pakistan

UNU-MERIT Dissertation Series, № 94

\section{Ajay Thutupalli}

Technology Paradigm Shifts in Agriculture:

Drivers of Sustainability and Catch up

UNU-MERIT Dissertation Series, № 93

\section{Eduardo Urias}

Improving access to HIV/AIDS treatment in

Brazil: When are Compulsory Licenses

effective in Price Negotiations?

UNU-MERIT Dissertation Series, № 92

\section{Francesca Guadagno}

Why have so few Countries Industrialised?

UNU-MERIT Dissertation Series, № 91 


\section{Daniel Opolot}

The Evolution of Beliefs and Strategic Behaviour

UNU-MERIT Dissertation Series, № 90

\section{Alejandro Lavopa}

Structural Transformation and Economic

Development: Can Development Traps be

Avoided

UNU-MERIT Dissertation Series, № 89

\section{Jinjin Zhao}

Urban water management reform: The Case of China

UNU-MERIT Dissertation Series, № 88

\section{Simona Vezzoli}

Borders, Independence and Post-colonial

Ties: the Role of the State in Caribbean

Migration

MGSoG Dissertation Series, № 65

Silvia Consuelo Gómez Soler

Civil Conflict and Education: How Does

Exposure to Civil Conflict Affect Human

Capital Accumulation? Evidence from

Standardized Exit Exams in Colombia

MGSoG Dissertation Series, № 64

\section{Paula Nagler}

Occupational Choice in the Developing

World

MGSoG Dissertation Series, № 63

\section{Jasmin Kientzel}

Determinants of Professional Commitment

to Environmental Sustainability

MGSoG Dissertation Series, № 62

\section{Mehmet Güney Celbiş}

Regional Policies: Convergence, Trade, and the Allocation of Public Capital

MGSoG Dissertation Series, № 61

\section{Florian Henning}

Living Up to Standard: Interoperability

Governance and Standards Adoption in

Government Information Networks

MGSoG Dissertation Series, № 60

\section{Niels P. Groen}

The Never-Ending Project

Understanding E-Government Project

Escalation

MGSoG Dissertation Series, № 59

\section{Derek Copp}

Teacher-Based Reactivity to Provincial

Large-scale Assessment in Canada

MGSoG Dissertation Series, № 58

\section{Michaella Vanore}

Family-Member Migration and the

Psychosocial Health Outcomes of Children

in Moldova and Georgia

MGSoG Dissertation Series, № 57

\section{Sonja Fransen}

The Economic and Social Effects of

Remittances and Return Migration in

Conflict-Affected Areas: The Case of

Burundi

MGSoG Dissertation Series, № 56

\section{Ibrahim Khalil Conteh}

The Impact of Floods on Primary School

Education in Zambia

MGSoG Dissertation Series, № 55 


\section{Richard Bluhm}

Growth Dynamics and Development

Essays in Applied Econometrics and

Political Economy

MGSoG Dissertation Series, № 54

\section{Nevena P. Zhelyazkova}

Work-Family Reconciliation and Use of

Parental Leave in Luxembourg: Empirical

Analysis of Administrative Records

MGSoG Dissertation Series, № 53

2014

\section{Dirk Crass}

The Impact of Brands on Innovation and

Firm Performance: Empirical Evidence from Germany

UNU-MERIT Dissertation Series, № 87

\section{Samyukta Bhupatiraju}

The Geographic Dimensions of Growth and Development

UNU-MERIT Dissertation Series, № 86

\section{François Lafond}

The Evolution of Knowledge Systems

UNU-MERIT Dissertation Series, № 85

\section{Annalisa Primi}

Promoting Innovation in Latin America:

What Countries Have Learned (and What

They Have Not) in Designing and

Implementing Innovation and Intellectual

Property Policies

UNU-MERIT Dissertation Series, № 84
Fatoumata Lamarana Diallo

Evaluation of Meal and Deworming

Programs for Primary Schools in Rural

Senegal

UNU-MERIT Dissertation Series, № 83

\section{Sachin Kumar Badkas}

Metachoice and Metadata: Innovating with

Environmental Policy Analysis in Europe

MGSoG Dissertation Series, № 52

\section{Irina S. Burlacu}

An Evaluation of Tax-Benefit Systems

Impact on the Welfare of Frontier Worker:

The Case of Luxembourg and Belgium

MGSoG Dissertation Series, № 51

\section{Özge Bilgili}

Simultaneity in Transnational Migration

Research: Links Between Migrants' Host

and Home Country Orientation

MGSoG Dissertation Series, № 50

\section{Yulia Privalova Krieger}

Reshaping the Big Agenda: Transnational

Politics and Domestic Resistance Financial

crisis and social protection reform in Bosnia and Herzegovina

MGSoG Dissertation Series, № 49

\section{Marieke van Houte}

Moving Back or Moving Forward? Return migration after Conflict

MGSoG Dissertation Series, № 48

\section{Oxana Slobozhan}

Global Governance in the Management of

Natural Resources: The Case of the

Extractive Industries Transparency

Initiative (EITI)

MGSoG Dissertation Series, № 47 


\section{Luis Bernardo Mejia Guinand}

The Changing Role of the Central Planning

Offices in Latin America: A Comparative

Historical Analysis Perspective (1950-2013)

MGSoG Dissertation Series, № 46

\section{Cheng Boon Ong}

Ethnic Segregation in Housing, Schools and

Neighbourhoods in the Netherlands

MGSoG Dissertation Series, № 45

\section{Luciana V. Cingolani}

Bureaucracies for Development: Oxymoron

or Reality? Studies on State Capacity in

Challenging Governance Contexts

MGSoG Dissertation Series, № 44

\section{Carlos Cadena Gaitán}

Green Politics in Latin American Cities -

Sustainable Transport Agendas

MGSoG Dissertation Series, № 43

\section{Katie Kuschminder}

Female Return Migration and Reintegration

Strategies in Ethiopia

MGSoG Dissertation Series, № 42

\section{Metka Hercog}

Highly-Skilled Migration and New

Destination Countries

MGSoG Dissertation Series, № 41

\section{Margaret Agaba Rugadya}

Can Remittances Influence the Tenure and

Quality of Housing in Uganda?

MGSoG Dissertation Series, № 40

\section{Ilire Agimi}

New Governance Under Limited Statehood:

The Case of Local Government Reform in

Kosovo

MGSoG Dissertation Series, № 39

2013

\section{Anant Kamath}

Information Sharing through Informal

Interaction in Low-Tech Clusters

UNU-MERIT Dissertation Series, № 82

\section{Flavia Pereira de Carvalho}

What we talk about when we talk about Brazilian Multinationals: An Investigation on Brazilian FDI, Economic Structure, Innovation and the Relationship between them

UNU-MERIT Dissertation Series, № 81

\section{Jun Hou}

Complementarity in Innovation and

Development: A Cross-country Comparison

UNU-MERIT Dissertation Series, № 80

\section{Rufin Baghana}

Impacts of Government Incentives to RED, Innovation and Productivity:

A Microeconometric Analysis of the Québec

Case

UNU-MERIT Dissertation Series, № 79

\section{Lilia I. Stubrin}

High-Tech Activities in Emerging

Countries: A Network perspective on the

Argentinean Biotech Activity

UNU-MERIT/MGSoG Dissertation

Series, № 78 


\section{Kristine Farla}

Empirical Studies on Institutions, Policies

and Economic Development

MGSoG Dissertation Series, № 38

\section{Marina Petrovic}

Social Assistance and Activation in the

Pursuit of Happiness: Shedding New Light on Old Policy Solutions to Social Exclusion MGSoG Dissertation Series, № 37

\section{Laura Torvinen}

Assessing Governance Assessments: The

Case of Mozambique: Governance

Assessments in the Context of Aid

Effectiveness Discourse

MGSoG Dissertation Series, № 36

\section{Biniam Egu Bedasso}

Institutional Change in the Long Shadow of Elite: Essays on Institutions, Human

Capital and Ethnicity in Developing

Countries

MGSoG Dissertation Series, № 35

\section{Sepideh Yousefzadeh Faal Deghati}

Childhoods Embargoed: Constructing and

Reconstructing Multidimensional Child

Poverty in Iran 1984-2009

MGSoG Dissertation Series, № 34

\section{Robert Bauchmüller}

Investing in Early Childhood Care and

Education: The Impact of Quality on

Inequality

MGSoG Dissertation Series, № 33

\section{Martin Rehm}

Unified Yet Separated: Empirical Study on

the Impact of Hierarchical Positions within

Communities of Learning

MGSoG Dissertation Series, № 32

2012

\section{Abdul Waheed}

Innovation Determinants and Innovation as a Determinant: Evidence from Developing Countries

UNU-MERIT Dissertation Series, № 77

\section{Bilal Mirza}

Energy Poverty and Rural Energy Markets in Pakistan

UNU-MERIT Dissertation Series, № 76

\section{Benjamin Engelstätter}

Enterprise Software and Video Games: An

Empirical Analysis

UNU-MERIT Dissertation Series, № 75

\section{Fulvia Farinelli}

Natural Resources, Innovation and Export Growth: The Wine Industry in Chili and Argentina

UNU-MERIT Dissertation Series

\section{Rodolfo Lauterbach}

Innovation in Manufacturing: From

Product Variety and Labor Productivity

Growth to Economic Development in Chile UNU-MERIT Dissertation Series

\section{Kirsten Wiebe}

Quantitative Assessment of Sustainable

Development and Growth in Sub-Saharan Africa

UNU-MERIT Dissertation Series, № 74 
Julio Miguel Rosa

Organizational Strategies, Firms'

Performance and Spatial Spillovers: The

Canadian Case in Research and

Development.

UNU-MERIT Dissertation Series, № 73

Johannes Wilhelmus Marie Boels

Joseph Schumpeter, Honderd Jaar

Economische Ontwikkeling: Een Historisch-

theoretische Beschouwing.

UNU-MERIT Dissertation Series

\section{Dorcas Mbuvi}

Utility Reforms and Performance of the

Urban Water Sector in Africa

MGSoG Dissertation Series, № 31

\section{Lina Salanauskaite}

Distributional Impacts of Public Policies:

Essays in Ex-Ante and Ex-Post Evaluation

MGSoG Dissertation Series, № 30

\section{Esther Schüring}

To Condition or not - is that the Question?

An Analysis of the Effectiveness of Ex-Ante

and Ex-Post Conditionality in Social Cash

Transfer Programs

MGSoG Dissertation Series, № 29

\section{Joe Abah}

Strong Organisations in Weak States:

Atypical Public Sector Performance in

Dysfunctional Environments

MGSoG Dissertation Series, № 28

\section{Zina Samih Nimeh}

Social Citizenship Rights: Inequality and

Exclusion

MGSoG Dissertation Series, № 27
2011

\section{Daniel Vertesy}

Interrupted Innovation: Emerging

Economies in the Structure of the Global

Aerospace Industry

UNU-MERIT Dissertation Series, № 72

\section{Tina Saebi}

Successfully Managing Alliance Portfolios:

An Alliance Capability View

UNU-MERIT Dissertation Series, № 71

\section{Nora Engel}

Tuberculosis in India: A Case of Innovation and Control

UNU-MERIT/MGSoG Dissertation

Series, № 70

\section{Evans Mupela}

Connectivity and growth in Sub-Saharan

Africa: The Role of Communication

Satellites

UNU-MERIT Dissertation Series, № 69

\section{Nantawan Kwanjai}

Cross Cultural Intelligence amid Intricate

Cultural Webs: A Tale of the UnDutchables in the Land of 1002 Smiles

UNU-MERIT Dissertation Series, № 68

\section{Lina Sonne}

Innovation in Finance to Finance

Innovation: Supporting Pro-poor

Entrepreneur-based Innovation

UNU-MERIT Dissertation Series, № 67

\section{Lenka Eisenhamerová}

Legitimacy of 'Humanitarian Military

Intervention'

MGSoG Dissertation Series, № 26 


\section{Sonila Tomini}

Informal Payments for Health Care Services in Albania

MGSoG Dissertation Series, № 25

\section{Jinjing Li}

Dynamic Microsimulation in Public Policy Evaluation

MGSoG Dissertation Series, № 24

\section{Aziz Atamanov}

Rural Nonfarm Employment and International Migration as Alternatives to Agricultural Employment: The Case of

Kyrgyzstan

MGSoG Dissertation Series, № 23

\section{Frieda Vandeninden}

Poverty Alleviation: Aid and Social

Pensions

MGSoG Dissertation Series, № 22

\section{Juliana Nyasha Tirivayi}

The Welfare Effects of Integrating AIDS

Treatment with Food Transfers: Evidence from Zambia

MGSoG Dissertation Series, № 21

\section{Agnieska Ewa Sowa}

Who's Left Behind? Social Dimensions of Health Transition and Utilization of Medical Care in Poland

MGSoG Dissertation Series, № 20

\section{Emmanaouil Sfakianakis}

The Role of Private Actors in the Provision of Public Goods with Applications to Infrastructure and Financial Stability MGSoG Dissertation Series, № 19

\section{Siu Hing Lo}

White Collars Green Sleeves: An Interorganizational Comparison of Determinants of Energy-Related Behaviors among Office Workers

MGSoG Dissertation Series, № 18

\section{Treena Wu}

Constraints to Human Capital Investment in Developing Countries:

Using the Asian Financial Crisis in Indonesia as a Natural Experiment

MGSoG Dissertation Series, № 17

\section{Henry Espinoza Peña}

Impact Evaluation of a Job-Training

Programme for Disadvantaged Youths:

The Case of Projoven

MGSoG Dissertation Series, № 16

2010

\section{Fernando Santiago}

Human Resources Management Practices and Learning for Innovation in Developing Countries: Pharmaceutical Firms in Mexico UNU-MERIT Dissertation Series, № 66

\section{Zakaria Babutsidze}

Essays on Economies with Heterogeneous

Interacting Consumers

UNU-MERIT Dissertation Series, № 65

\section{Bertha Vallejo}

Learning and Innovation Under Changing

Market Conditions: The Auto Parts

Industry in Mexico

UNU-MERIT Dissertation Series, № 64 


\section{Donatus Ayitey}

Technical Change, Competitiveness and

Poverty Reduction: A Study of the

Ghanaian Apparel Industry

UNU-MERIT Dissertation Series, № 63

\section{Sergey Filippov}

Multinational Subsidiary Evolution:

Corporate Change in New EU Member

States

UNU-MERIT Dissertation Series, № 62

\section{Asel Doranova}

Technology Transfer and Learning under the Kyoto Regime: Exploring the

Technological Impact of CDM Projects in

Developing Countries

UNU-MERIT Dissertation Series, № 61

\section{Florian Tomini}

Between Family and Friend: Understanding the Interdependency of Private Transfers

MGSoG Dissertation Series, № 15

\section{Michał Polalowski}

The Institutional Transformation of Social

Policy in East Central Europe: Poland and

Hungary in Comparative and Historical

Perspective

MGSoG Dissertation Series, № 14

\section{Maha Ahmed}

Defining, Measuring and Addressing

Vulnerability: The Case of Post Conflict

Environments

MGSoG Dissertation Series, № 13

\section{Pascal Beckers}

Local Space and Economic Success: The Role of Spatial Segregation of Migrants in the Netherlands

MGSoG Dissertation Series, № 12

\section{Victor Cebotari}

Conflicting Demands in Ethnically Diverse

Societies: Ethno political Contention and

Identity Values in Europe

MGSoG Dissertation Series, № 11

\section{Dennis Gyllensporre}

Competing and Complementary

Perspectives on the EU as a Crisis

Management Actor:

An Examination of the Common Security and Defence Policy through the Lenses of Idealism and Realism

MGSoG Dissertation Series, № 10

\section{Judit Vall Castello}

Business Cycle and Policy Effects on Labour

Market Transitions of Older and Disabled

Workers in Spain

MGSoG Dissertation Series, № 9

\section{Keetie Roelen}

False Positives or Hidden Dimensions: The

Definition and Measurement of Child

Poverty

MGSoG Dissertation Series, № 8

\section{Denisa Maria Sologon}

Earning Dynamics in Europe

MGSoG Dissertation Series, № 7

\section{Melissa Siegel}

Money and Mobility: Migration and

Remittances

MGSoG Dissertation Series, № 6 
Jessica S. Hagen-Zanker

Modest Expectations: Causes and Effects of

Migration on Migrant Households inSource Countries

MGSoG Dissertation Series, № 5

2009

\section{Alexis Habiyaremye}

From Primary Commodity Dependence to

Diversification and Growth: Absorptive

Capacity and Technological Catch Up in

Botswana and Mauritius.

UNU-MERIT Dissertation Series, № 60

\section{Yoseph Getachew}

The Role of Public Capital in Economic

Development

UNU-MERIT Dissertation Series, № 59

\section{Sandra Leitner}

Embodied Technological Change and

Patterns of Investment in Austrian

Manufacturing

UNU-MERIT Dissertation Series, № 58

\section{Semih Akçomak}

The Impact of Social Capital on Economic and Social Outcomes

UNU-MERIT Dissertation Series, № 57

\section{Abraham Garcia}

The Role of Demand in Technical Change

UNU-MERIT Dissertation Series, № 56

\section{Saurabh Arora}

Coherence in Socio-technical Systems: A

Network Perspective on the Innovation

Process

UNU-MERIT Dissertation Series, № 55

\section{Mirtha R. Muniz Castillo}

Human Development and Autonomy in

Project Aid: Experiences from four bilateral projects in Nicaragua and El Salvador

MGSoG Dissertation Series, № 4

\section{Christiane Arndt}

Governance Indicators

MGSoG Dissertation Series, № 3

\section{Britta Augsburg}

Microfinance: Greater Good or Lesser Evil?

MGSoG Dissertation Series, № 2

\section{8}

\section{Rutger Daems}

Medicines for the Developing World

UNU-MERIT Dissertation Series, № 54

\section{Johannes Hanel}

Assessing Induced Technology: Sombart's Understanding of Technical Change in the History of Economics

UNU-MERIT Dissertation Series, № 53

\section{Rifka Weehuizen}

Mental Capital: the Economic Significance of Mental Health

UNU-MERIT Dissertation Series, № 52

\section{Danielle Cloodt}

The Relationship between RED Partnership

Formation, Social Embeddedness and

Innovative Performance

UNU-MERIT Dissertation Series, № 51

\section{Sabine Fuss}

Sustainable Energy Development under

Uncertainty

UNU-MERIT Dissertation Series, № 50 


\section{Geranda Notten}

Measuring and Managing Poverty Risks

MGSoG Dissertation Series, № 1

2007

\section{Tobias Kronenberg}

Reconciling Environmental Conservation with Economic Prosperity: The Feasibility of

Double Dividends in the Short and Long Run

UNU-MERIT Dissertation Series, № 49

\section{Viktoria Kravtsova}

Assessing the Impact of Foreign Direct

Investment in Transition Economies

UNU-MERIT Dissertation Series, № 48

\section{Suhail Sultan}

The Competitive Advantage of Small and Medium Sized Enterprises: The Case of Jordan's Natural Stone Industry UNU-MERIT Dissertation Series, № 47

2006

\section{Bulat Sanditov}

Essays on Social Learning and Imitation UNU-MERIT Dissertation Series, № 46

\section{Mamata Parhi}

Dynamics of New Technology Diffusion: A Study of the Indian Automotive Industry UNU-MERIT Dissertation Series, № 45

\section{Andreas Reinstaller}

Social Structures and the Innovation

Process: Their Role in the Demand of Firms and Consumers

UNU-MERIT Dissertation Series, № 44

\section{Rose Kiggundu}

Innovation systems and Development: The Journey of a Beleaguered Nile Perch Fishery in Uganda

UNU-MERIT Dissertation Series, № 43

\section{Thomas Pogue}

The Evolution of Research Collaboration in South African Gold Mining: 1886-1933

UNU-MERIT Dissertation Series, № 42

\section{Geoffrey Gachino}

Foreign Direct Investment, Spillovers and Innovation: The Case of Kenyan

Manufacturing Industry

UNU-MERIT Dissertation Series, № 41

\section{Önder Nomaler}

Technological Change, International Trade and Growth: An Evolutionary, Multi-

Agents-Based Modeling Approach

UNU-MERIT Dissertation Series, № 40

\section{5}

\section{Samia Satti Osman Mohamed-Nour}

Change and Skill Development in the Arab Gulf Countries

UNU-MERIT Dissertation Series, № 39

\section{Elad Harison}

Intellectual Property Rights: Economics and Policy Analysis

UNU-MERIT Dissertation Series, № 38

\section{Daniel Dalohoun}

Learning to innovate: agricultural innovation and entrepreneurship: the case of Songhaï farmers in Benin

UNU-MERIT Dissertation Series, № 37 


\section{Müge Ozman}

Networks, Organizations and Knowledge

UNU-MERIT Dissertation Series, № 36

\section{Bas Straathof}

Product Variety and Economic Growth: The Counteracting Effects of Scale and

Idiosyncrasy

UNU-MERIT Dissertation Series, № 35

\section{Wilfred Schoenmakers}

Knowledge Flows between Multinational

Companies: A Patent Data Analysis

UNU-MERIT Dissertation Series, № 34

\section{Myriam Cloodt}

Mergers and Acquisitions ( $M$ and $A s$ ) in

High-Tech Industries: Measuring the Post$M$ and $A$ Innovative Performance of

Companies

UNU-MERIT Dissertation Series, № 33

\section{4}

\section{Paola Criscuolo}

$R \mathcal{E} D$ Internationalisation and Knowledge

Transfer: Impact on MNEs and their Home Countries

UNU-MERIT Dissertation Series, № 32

\section{Maarten Verkerk}

Trust and Power on the Shop Floor

UNU-MERIT Dissertation Series, № 31

\section{Gottfried Leibbrandt}

Adoption, Harmonization and Succession of Network Technologies across Countries UNU-MERIT Dissertation Series, № 30

\section{Mark Sanders}

Skill Biased Technical change: Its Origins, the Interaction with the Labour Market and Policy Implications

UNU-MERIT Dissertation Series, № 29

2003

\section{Nadine Roijakkers}

Inter-firm Cooperation in High-tech

Industries: a Study of RED Partnerships in

Pharmaceutical Biotechnology

UNU-MERIT Dissertation Series, № 28

\section{Viki Sonntag}

Speed, Scale and Sustainability

UNU-MERIT Dissertation Series, № 27

\section{Masaru Yarime}

From End-of-Pipe Technology to Clean

Technology

UNU-MERIT Dissertation Series, № 26

\section{Stéphane Malo}

The Combinatorial Chemistry Revolution:

Sustaining a Superior Performance Position through Technological Learning

UNU-MERIT Dissertation Series, № 25

\section{2}

\section{Annelies Hogenbirk}

Determinants of Inward Foreign Direct Investment: the Case of the Netherlands UNU-MERIT Dissertation Series, № 24

\section{Bastiaan Johan terWeel}

The Computerization of the Labour Market UNU-MERIT Dissertation Series 


\section{John Adeoti}

Technology Investment in Pollution Control in Sub-Saharan Africa: The Case of the Nigerian Manufacturing Industry UNU-MERIT Dissertation Series, № 23

\section{Edward Huizenga}

Innovation Management: How

Frontrunners Stay Ahead: An Empirical

Study on Key Success Factors in the ICT sector

UNU-MERIT Dissertation Series, № 22

2000

\section{Machiel van Dijk}

Technological Change and the Dynamics of Industries: Theoretical Issues and Empirical evidence from Dutch Manufacturing

UNU-MERIT Dissertation Series, № 21

1999

\section{Jan Cobbenhagen}

Managing Innovation at the Company

Level: A Study on Non-Sector-Specific

Success Factors

UNU-MERIT Dissertation Series, № 20

\section{Marjolein Caniëls}

Regional Growth Differentials: The Impact of Locally Bounded Knowledge Spillovers UNU-MERIT Dissertation Series, № 19

\section{Aldo Geuna}

Resource Allocation and Knowledge

production: Studies in the Economics of University Research

UNU-MERIT Dissertation Series, № 18

1996

\section{Reinoud Joosten}

Dynamics, Equilibria, and Values

UNU-MERIT Dissertation Series, № 17

\section{Hugo Kruiniger}

Investment, $R \mathcal{E} D$, and the Financing

Decisions of the Firm

UNU-MERIT Dissertation Series, № 16

1995

\section{Hans van Meijl}

Endogenous Technological Change: The

Case of Information Technology, Theoretical

Considerations and Empirical Results

UNU-MERIT Dissertation Series, № 15

\section{René Kemp}

Environmental Policy and Technical

Change: A Comparison of the Technological Impact of Policy Instruments

UNU-MERIT Dissertation Series, № 14

\section{Rohini Acharya}

The Impact of New Technologies on Economic Growth and Trade: A Case Study of Biotechnology

UNU-MERIT Dissertation Series, № 13 


\section{Geert Duysters}

The Evolution of Complex Industrial

Systems: The Dynamics of Major IT Sectors

UNU-MERIT Dissertation Series, № 12

\section{Marjan Groen}

Technology, Work and Organisation: A

Study of the Nursing Process in Intensive

Care Units

UNU-MERIT Dissertation Series, № 11

1994

\section{Huub Meijers}

On the Diffusion of Technologies in a

Vintage Framework: Theoretical

Considerations and Empirical Results

UNU-MERIT Dissertation Series, № 10

\section{Theon van Dijk}

The Limits of Patent Protection: Essays on the Economics of Intellectual Property

Rights

UNU-MERIT Dissertation Series, № 9

\section{Hans Voordijk}

Naar Integrale Logistiek in Bedrijfsketens:

Ontwikkelingen in de Bouw

UNU-MERIT Dissertation Series, № 8

1993

\section{Paul Diederen}

Technological Progress in Enterprises and Diffusion of Innovation: Theoretical Reflections and Empirical Evidence

UNU-MERIT Dissertation Series, № 7

\section{Ben Dankbaar}

Economic Crisis and Institutional Change:

The Crisis of Fordism from the Perspective of the Automobile Industry

UNU-MERIT Dissertation Series, № 6

\section{Hanno Roberts}

Accountability and Responsibility: The

Influence of Organisation Design on

Management Accounting

UNU-MERIT Dissertation Series, № 5

1992

\section{Bart Verspagen}

Uneven Growth between Interdependent

Economies: An Evolutionary View on

Technology Gaps, Trade and Growth

UNU-MERIT Dissertation Series, № 4

\section{Sjoerd Romme}

A Self-organization Perspective on Strategy

Formation

UNU-MERIT Dissertation Series, № 3

1989

\section{John Spangenberg}

Economies of Scale, and Atmosphere in

Research Organisations

UNU-MERIT Dissertation Series, № 2

1988

\section{John Hagedoorn}

Evolutionary and Heterodox Innovation

Analysis: A Study of Industrial and

Technological Development in Process

Control and Information Technology

UNU-MERIT Dissertation Series, № 1 